The Structure of Adolescent and Adult Savoring and its Relationship to Feeling Good and Functioning Well

By

Erica D. Chadwick

\begin{abstract}
A thesis submitted for the degree of Doctor of Philosophy in Psychology at Victoria University of Wellington, Wellington, New Zealand.
\end{abstract}


I sought to contribute to the understanding of positive health, in particular savoring and wellbeing, by conducting concurrent and longitudinal studies with adolescents and adults. The thesis begins with a review of the literature including savoring theory (Bryant \& Veroff, 2007) and the broaden-and-build theory of positive emotions (Fredrickson, 2001); these theories led to the key expectations that the psychometric structure of everyday savoring would be similar for adolescents and adults, and that amplifying savoring would positively predict wellbeing. Data obtained from two surveys, a paper-and-pencil survey with New Zealand adolescents (13 to 15 years old) and an internet-based survey with international adults (16 to 88 years old), were investigated in four studies across four empirical chapters (Chapters 2 through 5).

Study 1 (Chapter 2) explored the similarities and differences in the psychometric structure of an abridged Ways of Savoring Checklist, labelled everyday savoring, between adolescents $(N=463)$ and adults $(N=980)$, as well as mean group differences in adolescents' and adults' degree of savoring. Study 2 (Chapter 3) investigated the concurrent relationships between adolescent and adult everyday savoring and hedonic and eudaimonic wellbeing as well as the ability of savoring to moderate wellbeing. Then, Study 3 and Study 4 investigated the relationships between savoring and wellbeing across time for adolescents ( $N=265$; Study 3 , Chapter 4) and adults ( $N=1858$; Study 4, Chapter 5), including savoring as a mediator of the relationship between everyday positive events and wellbeing (Chapter 4), and orientations to happiness as a moderator of savoring and everyday positive events (Chapter 5).

Results indicated that adolescents and adults yielded a similar four-factor structure of everyday savoring: dampening ("I don't deserve it"), low arousal ("I tried to slow down"), high arousal ("I jumped up and down"), and self-focus ("I reminded myself how lucky I was") savoring strategies, which proved to be invariant across time. The adolescent group, however, manifested a stronger association between amplifying (i.e. low arousal, high arousal, and selffocused savoring) and dampening savoring. Adolescents also reported higher levels of dampening compared to the adult group, whereas adults reported higher amplifying than adolescents. As expected, high arousal and self-focused savoring were positively, and dampening was negatively, associated with wellbeing indicators for adolescents and adults. However, low arousal savoring was negatively associated with hedonia for adolescents, but positively associated with eudaimonia for adults. The longitudinal analyses indicated that amplifying savoring predicted increases in wellbeing whereas dampening savoring predicted decreases in wellbeing for both age groups. The direction of effect, however, was not always as expected, questioning 
general assumptions of savoring theory and the broaden-and-build theory of positive emotions. These exceptions are most noted and explored in the final empirical chapter, Chapter 5.

Overall the findings suggest that savoring is similar and similarly important for wellbeing over the age range incorporating adolescence to adulthood, although potential developmental differences are important to consider. The contribution of this thesis to the study of savoring, the field of positive psychology, and positive health development are reviewed in Chapter 6, as are the implications, limitations, and future directions. 


\section{Acknowledgements}

My thesis is not an accomplishment I achieved in isolation, although I would rather not attempt to count how many days I felt like I was living in a cave. The truth is that without my supervisor, mentors, participants, family, friends, and colleagues contributing to my work and shining light into my metaphorical cave, minor hurdles would have appeared to be unsurpassable mountains.

To begin, the backbone of this thesis is the schools, principals, counsellors, adolescents and international adults who were interested and participated in my studies on multiple occasions. I hope that my research will in some small way add to the efforts of positive psychology to make their lives fuller.

I owe thanks to the Royal Society of New Zealand for generously supporting my research and Victoria University for supporting my participation in two eye-opening conferences. Thank you both. I owe my gratitude specifically to the administration and technical staff at Victoria University: Ngaire, Nicola, Jebi, Wendy, Doug Drysdale, Michael, and Vijay. Their assistance with all my slightly panicked requests and form phobia was immensely appreciated.

I was incredibly fortunate to have an overwhelmingly positive $\mathrm{PhD}$ experience, and this is greatly due to my outstanding and attentive supervision by Associate Professor Paul Jose. Paul taught me the wonderful world of statistical equation modelling with amazing patience and simplicity. He has encouraged me as a researcher, teacher, mentor, public speaker and writer-all skills I know will hold me well throughout my career. Professor Fred B. Bryant was also instrumental in the development of my abilities as a researcher. Phone calls and a summer lunch in Leadville Colorado with Fred stimulated my enthusiasm for research and graced me with unmatched words of motivation when I most needed them. I can only hope to be as patient, encouraging and inspiring to others about psychology and savoring as Paul and Fred are for me.

I am also indebted to Aaron Jarden and grateful to know his inspiration, accessibility, and his tireless efforts spearheading the International Wellbeing Study. I know that if I am able to adopt even an ounce of his passion and drive my career will positively progress for years to come.

I am honoured that during this process I was supported by remarkable friends. In particular, Robyn Langlands brought me perspective and patience when I misplaced mine. And Gemma Sheehan provided me with reliably sound and subtle advice that imparted me with tangible strategies for, figuratively, "putting one foot in front of the other." I feel privileged to be acquainted with their encouragement and I look forward to sharing many more of our 
experiences. I hope that I support and encourage others as well as I have been supported and encouraged by Robyn and Gemma.

I have saved those that mean the most to me for last. My mother, Theresa Nieslanik, has unwaveringly supported me since day one. Watching her create purpose and meaning from her brightest and darkest moments taught me to approach my $\mathrm{PhD}$ pragmatically and in an engaged manner. Although my sister, Melinda, is no longer here, the strength and fortitude I learned from her kept me steadily moving toward this goal. My in-laws, Steve and John Chadwick, have provided me with more than I could ever ask. With their support I have more than survived, I flourished as a PhD candidate, while being a wife and mother. My husband, Rama Chadwick, has been by my side most of all. He endured the slumps and celebrated the highs. He snuck in timely doses of reality (and cups of tea) and always reminded me I could do it. I am so grateful for each moment I shared throughout this process with Rama and our stunning children-Manaia Te Ataiwaho Makani and Kurai Te Manihera. This thesis is dedicated to you, my boys, and to all the moments we get to savor together. 


\section{Table of Contents}

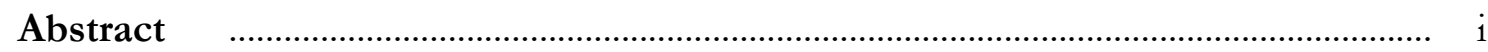

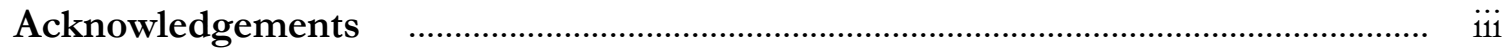

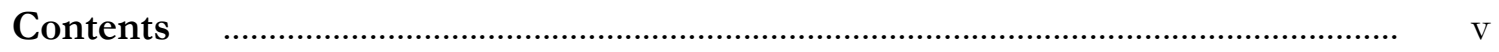

List of Tables

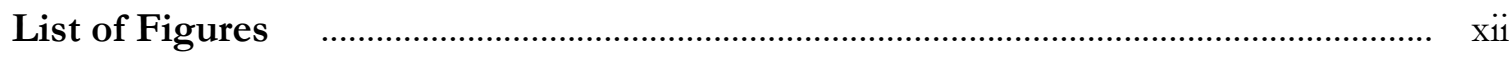

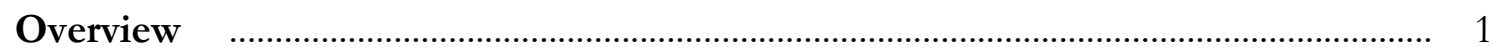

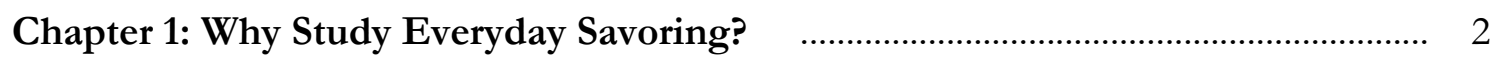

The birth of Positive Psychology $\quad$ _........................................................................ 2

Definitions of happiness $\quad$..................................................................................... 4

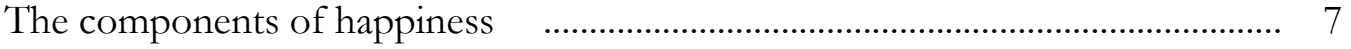

The benefits of happiness $\quad$...................................................................................... 12

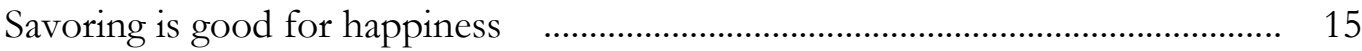

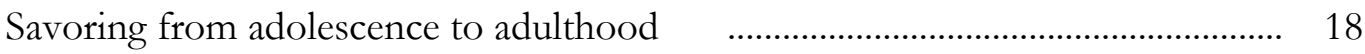

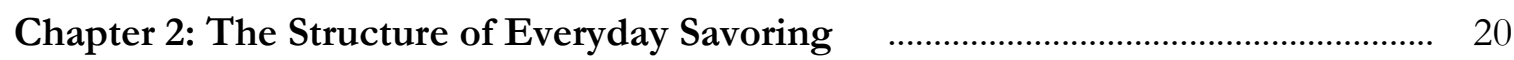

The Ways of Savoring Checklist $\quad$........................................................................ 20

The savoring strategies: How positive events and affect are enhanced and prolonged

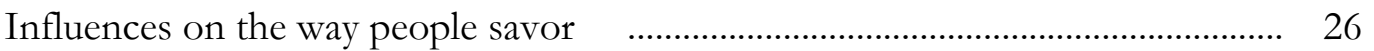

Hypotheses: Further directions in the investigation of savoring strategies $\quad$...... 29

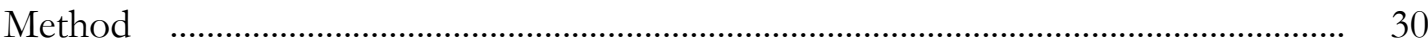

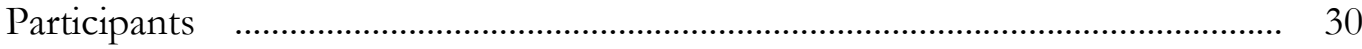

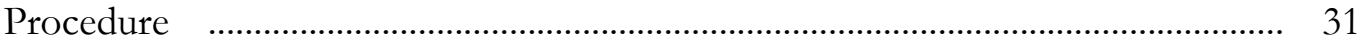

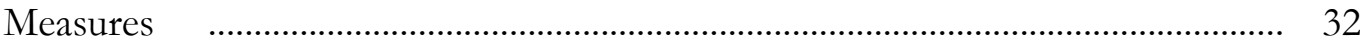

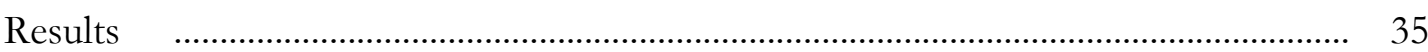

Analytic Strategy $\quad$............................................................................................ 35 
Factorial validity/configural analysis: Is the model of savoring everyday positive events similar for adolescents and adults?

Structural Invariance: Are there differences in the multi-dimensional nature of savoring strategies between adolescents and adults?

Gender and Age: Do gender and age influence savoring strategies?

Discussion

Hypothesis 1: The configuration of everyday savoring of everyday positive events

Hypothesis 2: The Structure of Savoring Everyday Positive Events

Hypotheses 3 through 5: How gender and age influence the savoring of everyday positive events

\section{Chapter 3: Concurrent Savoring and Wellbeing}

Brief review: Indicators of wellbeing

Savoring's association with wellbeing

Does age moderate the relationship between savoring and wellbeing?

Hypotheses: Validity of adolescent and adult everyday savoring

Method

Participants and Procedure

Measures

Results

Analytic Strategy

Non-parametric tests: Do adolescents and adults similarly rank their endorsement of savoring strategies?

Discriminant Validity: Do savoring strategies uniquely relate to wellbeing?

Do savoring strategies moderate the relationship from hedonic to eudaimonic wellbeing?

Discussion

Hypotheses 1 and 2: Ranking of the four everyday savoring strategies $\quad$............ 77

Hypotheses 3 and 4: Savoring Strategies: Unique predictors of wellbeing $\quad \ldots \ldots . . . \quad 78$ 
Hypotheses 5 and 6: Savoring predictors and wellbeing and the influence of age

Hypotheses 7 through 10: Moderating the relationship from hedonic to eudaimonic wellbeing

Chapter 4: Adolescent Savoring and Wellbeing over Time

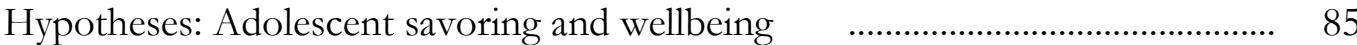

Method

Participants and Procedure

Measures

Results

Analytic Strategy

Reliability and validity: Are the four factors of savoring stable over time and would concurrent relationships be replicated across time?

Are savoring strategies predictors of positive affect and eudaimonia over time, and is the savoring and positive affect relationship bi-directional?

Structural mediational models: Are there mediating relationships between positive life events, savoring, positive affect, and eudaimonia concurrently and over time?

Discussion

Hypotheses 1 through 4: Psychometric tests of savoring

Hypotheses 5 through 8: Savoring strategies: Predictors of positive affect and eudaimonia

Hypotheses 9 through 11: Savoring and positive affect: Concurrent and longitudinal mediators

\section{Chapter 5: Adult Savoring and Wellbeing over Time}

Hedonic and eudaimonic orientations to happiness as moderators of savoring .. 115

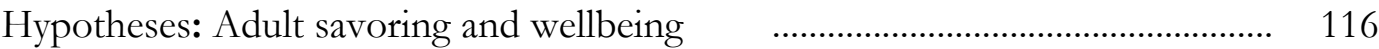

Method

Participants

Procedure 
Results

Analytic Strategy

Confirmation of findings from adolescent savoring with adults: The reliability and validity of savoring

Developmental trends in savoring, hedonic and eudaimonic orientations to happiness, satisfaction with life, and everyday positive life events

The directions of effect between savoring, hedonic and eudaimonic orientations to happiness, satisfaction with life, and everyday positive life events across three months

The moderating influence of pleasure and meaning orientations to happiness on savoring

Discussion

Hypotheses 1 through 4: Psychometric tests of savoring

Hypotheses 5 through 7: Developmental differences in savoring, wellbeing, and positive events

Hypotheses 8 through 11: The direction of effect between savoring, wellbeing, and positive events

Exploratory analyses and findings: Bi-directional relationships

Hypotheses 12 and 13: The moderating influence of pleasure and meaning orientations to happiness on savoring

\section{Chapter 6: Contributing to Savoring and Wellbeing Research}

Key Findings

Similarities and differences amongst savoring strategies across age

Savoring and everyday positive events

Savoring and subjective wellbeing

Strengths and Implications

Everyday savoring

Everyday savoring and subjective wellbeing 
Appendix A: Original Ways of Savoring Checklist

Appendix B: Everyday Positive Events Scale (Adolescents)

Appendix C: Everyday Positive Events Scale (Adults) 186

Appendix D: Abridged Ways of Savoring Checklist (Adolescents) 187

Appendix E: Abridged Ways of Savoring Checklist (Adults)

Appendix F: Satisfaction with Life Scale

Appendix G: Temporal Satisfaction with Life Scale

Appendix H: Subjective Happiness Scale

Appendix I: Mental Health Short Form 194

Appendix J: Orientations to Happiness Scale

Appendix K: Positive Negative Affective Schedule 


\section{List of Tables}

Cronbach's Alpha and Number of Items for each Dimension of the

Table 2.1 Original 60-item WOSC for Young Adults, and the 20-item Abridged

WOSC used with Adolescents and Adults

Table 2.2 20 Items from the Abridged Ways of Savoring Checklist

Goodness-of-fit Statistics for CFA Measurement Models of the 20-item

Table 2.3 WOSC in Response to Everyday Events, Adolescent $(N=463)$ and Adult

$(N=980)$ samples

Table 2.4 Factors, Cronbach's Alphas and Items for the Four-Factor Model of the Abridged WOSC in Response to Everyday Events

Table 2.5 Goodness-of-Fit statistics for Tests of Invariance across Adolescent $(N=$ 463) and Adult Samples $(N=980)$ : A Summary

Table 2.6
$=332)$ on Four Factors of Savoring

Summary of Wilcoxon's Signed-Rank Results: Adolescent $(N=463)$ and

Table 3.1 Adult $(N=980)$ Rankings of Dampening, High Arousal, Low Arousal, and

Self-focus Savoring

Table 3.2 The Impact of Hedonic Wellbeing on Eudaimonic Wellbeing, moderated by Low Arousal Savoring, and High Arousal Savoring

Demographic Information Comparing the Correlational and Longitudinal

Table 4.1 Adolescent Studies

Table 4.2 Concurrent Correlations among Latent Variables for Baseline and 4-week Measurement Models of Savoring, Positive Affect, and Eudaimonia

Summary of Model Fit for Saturated and Pruned Mediational Models with

Table 4.3 $\begin{aligned} & \text { Summary of Model Fit } \\ & \text { Mediators at Time } 1\end{aligned}$

Summary of Model Fit for Saturated and Pruned Mediational Models with Mediators at Time 2

Table 4.5 Summary of Tests of Invariance by Gender 108

Table 5.1 Summary of Correlations between the Four Factors of Everyday Savoring ..

Table 5.2

Time 1 Goodness-of-Fit Statistics for Tests of Invariance of Dampening Covariances across Young, Middle, and Older Adults 
Means and Standard Deviations for Savoring, Orientations to Pleasure and

Table 5.3 Meaning, Satisfaction with Life, and Everyday Positive Life Events

Averaged across 3 Months for Young, Middle, and Older Adults

Table 5.4 Correlations among Latent Variables for Baseline and 3-month Measurement Models

Standardised Regression Coefficients for the Savoring, Hedonic,

Table 5.5 Eudaimonic, Satisfaction with Life, and Everyday Positive Life Events

Pruned Structural Path Model

Table 5.6 The Impact of Savoring on Positive Events Moderated by Meaning

Orientation 


\section{List of Figures}

Figure 1.1 Three levels of happiness: Integrating hedonia and eudaimonia $\quad$.................... 5

Figure 1.2 Three levels of savoring $\quad$.............................................................................. 16

Figure 2.1 Four-factor model of savoring strategies (standardised estimates) $\quad$............... 42

Figure 2.2 Correlations between dampening savoring and low arousal, high arousal, and self-focus savoring for adolescents and adults

Figure 2.3 The interaction between gender and age on dampening Savoring

Figure 2.4 Dampening, high arousal, and self-focus everyday savoring strategies reported by adolescents and adults

Figure 3.1 Adolescents' and adults' mean ranked endorsement for the four savoring
strategies: Dampening, low arousal, high arousal, and self-focus savoring ......

Figure 3.1 Adolescents' and adults' mean ranked endorsement for the four savoring
strategies: Dampening, low arousal, high arousal, and self-focus savoring .....

Figure 3.2 Standardised adolescent (Model A, $N=463$ ) and adult (Model B, $N=980$ ) measurement models of satisfaction, happiness and eudaimonia

Standardised path models of adolescent (Model A, $N=463$ ) and adult

Figure 3.3 (Model B, $N=980$ ) everyday savoring strategies and their relationship to satisfaction, happiness, and eudaimonia

Figure 3.4 Low arousal savoring moderating the impact of hedonic wellbeing on eudaimonic wellbeing for adolescents (Model A) and adults (Model B)

Figure 4.1 Pruned model of savoring, positive affect, and eudaimonia over four weeks

Figure 4.2 The standardised pruned model for self-focus savoring and positive affect over four weeks

Figure 4.3 Fully saturated mediational model of everyday positive events, dampening and self-focus savoring, positive affect, and eudaimonia

Figure 4.4 Pruned concurrent mediational path models of positive life events, savoring, positive affect, and eudaimonia

Figure 4.5 Indirect effects for the pruned concurrent mediational model of positive life events, self-focus savoring, positive affect, and eudaimonia

Figure 4.6 Summary of the pruned and standardised longitudinal residualised mediational path models with mediators at Time 1

Figure 4.7 Summary of Longitudinal residualised mediational path models with mediators at Time 2 
Figure 5.1 Age distribution of the study sample

The correlations between dampening and low arousal savoring at Time 1

Figure 5.2 (left model) and Time 2 (right model) for young (top value) and middle aged adults (bottom value)

Reported rates of everyday savoring strategies, pleasure and meaning

Figure 5.3 orientations to happiness, satisfaction with life, and everyday positive events by young $(15-30 \mathrm{yrs})$, middle $(31-49 \mathrm{yrs})$, and older adults $(50-88 \mathrm{yrs}) \ldots$

Pruned direction of effect model: Savoring, pleasure and meaning

Figure 5.4 orientations to happiness, satisfaction with life, and everyday positive events across three months $\left(\chi^{2} / \mathrm{df}\right.$ ratio $\left.=2.74, \mathrm{CFI}=.96, \mathrm{RMSEA}=.03, \mathrm{sRMR}=.04\right)$

Figure 5.5 Final bi-directional models

Figure 5.6 Savoring at Time 1 and everyday positive life events at Time 2 moderated by average meaning orientation to happiness 


\title{
O TASTE AND SEE
}

\author{
The world is \\ not with us enough \\ $O$ taste and see \\ the subway Bible poster said, \\ meaning The Lord, meaning \\ if anything all that lives \\ to the imagination's tongue, \\ grief, mercy, language, \\ tangerine, weather, to \\ breathe them, bite, \\ savor, chew, swallow, transform \\ into our flesh our \\ deaths, crossing the street, plum, quince, \\ living in the orchard and being \\ hungry, and plucking \\ the fruit. \\ 〜Denise Levertov, (1964)
}




\section{Overview}

The field of positive psychology studies the "good" things in life, for example, positive emotions, happiness, life satisfaction, and meaning. A major process that promotes attention to, appreciation of, and enhancement of these positive experiences is savoring (Bryant \& Veroff, 2007).

The process of savoring is constituted by ten, empirically supported, ways of savoring (e.g. counting blessings, sharing with others) that are proposed to moderate the quality of positive experience and mediate relationships with positive affect (Bryant, Ericksen, \& DeHoek, 2008; Bryant \& Veroff, 2007). This thesis aims to: uncover the similarities and differences between adolescent and adult savoring strategies (Chapter 2); examine savoring similarities and differences between adolescent and adult savoring in relation to wellbeing, as well as examine the ability of savoring to moderate wellbeing (Chapter 3); test the direction of effect between positive events, savoring, and wellbeing for adolescents (Chapter 4) and adults (Chapter 5); and test the ability of savoring to mediate positive events and wellbeing (Chapter 4), and the ability of wellbeing to moderate savoring (Chapter 5). It is hoped that the findings from these studies will reveal specific savoring strategies for leading happier and more fulfilling lives from adolescence to late adulthood.

Happy people are more successful in all life domains including, family, friends, work, and health (Lyubomirsky, King, \& Diener, 2005). Savoring is proposed to be a central mechanism for engendering these positive outcomes-it follows that researching savoring will illuminate pathways for promoting healthy, happy, successful people.

My interest in the cognitive-behavioural strategies that regulate positive experience stems from my desire to provide a positive balance to my clinical training. I seek to understand how individuals savor on an everyday basis in order to contribute to the development of further research, including prevention and intervention studies with clinical populations.

Researchers maintain that the ability to handle adversity is vital to living, although not the same as the capacity to enjoy living (e.g. Watson, 2000b). Focusing on how to ameliorate problems may prevent the proverbial glass from appearing empty, but it will require an understanding of the mechanisms and pathways of positive processes to add to and savor what's in the glass. 


\section{CHAPTER 1}

\section{Why Study Everyday Savoring?}

The more we learn about man's natural tendencies, the easier it will be to tell him how to be good, how to be happy, how to be fruitful, how to respect himself, how to love, bow to fulfil his highest potentialities.

(Abraham Maslow, 1962, p. 4)

The desire to attain happiness is as old as recorded history. The interest in defining happiness and how to acquire it has been documented in both man's earliest stories and the intellectual postulations of philosophers (Cahn \& Vitrano, 2008; McMahon, 2006). Socrates, Plato, and Aristotle proposed happiness was the ultimate desire and the final end. Since World War II (WWII) the majority of psychological research, in contrast, has focused on unhappiness and maladaptation (when wellbeing has been considered, it has largely been to understand the impact of negative factors, for instance, stressful events). Although the relationship between negative predictors and wellbeing has been of great interest, the relationships between positive events, positive processes and wellbeing have been relatively neglected. For instance, coping with stress has been and continues to be well researched (Ein-Dor, Mikulincer, \& Shaver, 2011; Folkman \& Lazarus, 1985a; Folkman, Lazarus, Gruen, \& DeLongis, 1986), while savoring positive experiences has had scant coverage in comparison (Bryant \& Veroff, 2007; Quoidbach, Berry, Hansenne, \& Mikolajczak, 2010). A push has come from the sub-discipline within psychology known as positive psychology to again focus on what makes life most worth living. Moreover, a complete study of psychology must include the study of healthy human functioning.

\section{The birth of Positive Psychology}

Prior to the Second World War, psychology had three distinct missions: (1) curing mental illness, (2) identifying and nurturing high talent, and (3) making the lives of all people more productive and fulfilling (Seligman, 2002; Seligman \& Csikszentmihalyi, 2000; Seligman, Parks, \& Steen, 2004). The impetus of the infant discipline of psychology in the early $19^{\text {th }}$ century was to study healthy functioning and human dysfunction with equal priority (Brennan, 2009). Into the first few decades of the $20^{\text {th }}$ century, psychological researchers investigated positive traits (e.g. giftedness), adaptive behavioural functioning (e.g. marital harmony/status, effective parenting), and positive cognitive processes (e.g. finding meaning and purpose) (Jones, 1966).

A shift came with WW II that narrowed the focus of psychology. The influx of war veterans during, and post-WW II, experiencing acute distress put demands on psychiatry that outweighed its capacity. The Veterans Administration Act of 1946 and the Boulder conference 
of 1949 were two initiatives that responded with objectives to increase the postgraduate training of professional psychologists with the main purpose of providing therapy to veterans (Baker \& Benjanin, 2000; Seligman, 2002). Economic incentives to study pathology and the amelioration of dysfunction prompted researchers to turn away from studying adaptive functioning and the development of positive traits in favour of assessing and addressing how individuals cope with difficult conditions. By and large, the funding for psychological research and training went from supporting three missions (listed above) to supporting just one: curing mental illness. The result was a proliferation of applied science and the disease model within the discipline of psychology. Since this watershed period, for every article on satisfaction, happiness, and joy, there have been 21 articles on anger, anxiety, and depression (Ben-Shahar, 2007). This virtually singular focus has made great gains in uncovering the impact of stressors on cognitive and emotional functioning as well as the various strategies individuals implement to cope with adversity. Assumptions were made, however, that by treating disorder, disorder would be prevented, and that by treating dysfunction, adaptive functioning would spontaneously result (Seligman, 2002).

Several researchers questioned these assumptions by proposing theories and gathering evidence for fostering positive functioning, positive wellbeing, and mental health (Bradburn, 1969; Fordyce, 1977; Jahoda, 1958; Maslow, 1954, 1962). A subsequent explosion in emotion research during the 1980s and 1990s uncovered new evidence for state mood fluctuations and individual differences in temperament and emotionality. A leading finding from these two decades of emotion research showed that positive and negative affective systems are relatively independent—-both vital contributors to our mood and emotionality (Bradburn, 1969; Diener \& Emmons, 1985; Watson, 2000a) (for a competing view, see Barrett \& Russell (1998)). These findings added weight to the criticisms made by Maslow, Jahoda, and others by arguing that the systems related to managing adversity are essential to living, but they are not identical to the systems necessary for enjoying living. In other words, another set of tools is required for living the "good life" separate and different from the tools required for ameliorating hardship, and these positive traits, states, and abilities deserve equal attention.

Researchers and the mounting literature base pushed psychology to return a balance to the discipline by boosting its focus on positive functioning. The drive for this shift culminated in what is most widely recognized as the birth of Positive Psychology: Martin Seligman's 1998 APA presidential address. This was followed by the seminal issue of American Psychologist in 2000, which was dedicated to the emerging science of Positive Psychology, in other words, the 
scientific research of what constitutes fulfilling and meaningful lives, thriving communities, and resilient individuals (Seligman \& Csikszentmihalyi, 2000).

Since then, two peer-reviewed journals devoted to the scientific investigation of subjective wellbeing were formed-the Journal of Happiness Studies and the Journal of Positive Psychology. In 2005 the inaugural edition of the Handbook of positive psychology was published, solidifying the discipline, and reporting the reputable theoretical and empirical progress thus far featuring chapters on the benefits of sharing with others (Niederhoffer \& Pennebaker, 2005), positive emotions and positive affectivity (Fredrickson, 2005b; Watson, 2005), positive child development and prevention (Roberts, Brown, Johnson, \& Reinke, 2005), and working toward a science of mental health (Keyes \& Lopez, 2005). Further, the field of positive psychology continues to support the in depth examination of numerous topics including gratitude (Emmons \& McCullough, 2003; Emmons \& Shelton, 2002; Froh, Sefick, \& Emmons, 2008; McCullough, Emmons, \& Tsang, 2002; McCullough, Tsang, \& Emmons, 2004), optimism (Bryant \& Cvengros, 2004; Carver, Scheier, \& Segerstrom, 2010; Palgi, Shrira, Ben-Ezra, Cohen-Fridel, \& Bodner, 2011; Seligman, 1992), happiness (Lyubomirsky, 2008; Lyubomirsky, Sheldon, \& Schkade, 2005), life satisfaction (Diener, Emmons, Larsen, \& Griffin, 1985; Pavot \& Diener, 2008, 2009), psychological wellbeing (Ryff, 1989; Ryff \& Keyes, 1995; Ryff \& Singer, 2008), and a dual model of mental health (Keyes, 2002, 2003, 2007; Suldo \& Shaffer, 2008; Suldo, Thalji, \& Ferron, 2011) — to name a few. It follows that the topic of the present thesis, i.e., cognitivebehavioural strategies that work to build wellbeing by engendering, attending, enhancing, and appreciating positive experiences (i.e. savoring), is at home among these inspiring topics of study, and is central to the field of positive psychology.

\section{Definitions of happiness}

Happiness has been difficult for psychologists to define (Kennedy, 2011). The general definition is wide reaching, often making the boundaries of the construct murky and indistinct. Some researchers describe a hedonic view of happiness while others describe a more integrated view of hedonia and eudaimonia (for an overview of hedonic psychology see, Kahneman, Diener, \& Schwarz, 1999). The theoretical rationale for hedonic wellbeing is that individuals act to maximise pleasure and reduce pain, whereas the theoretical rationale for eudaimonic wellbeing is that people strive to live by personally adopted values (Ryan \& Deci, 2001). Recently Seligman has proposed understanding the fuzzy notion of happiness as three more scientifically manageable components: positive emotions (the pleasant life), engagement (the engaged life), and meaning (the meaningful life) (Peterson, Park, \& Seligman, 2005; Seligman, 2002; Seligman, 
et al., 2004). Another view, proposed by Nettle (2005), is a model where happiness is composed of three levels that include hedonia and eudaimonia. As shown in Figure 1.1, each increasing level of happiness includes the previous level plus additional elements.

\begin{tabular}{ccc}
\hline Level 1 & Level 2 & Level 3 \\
\hline Momentary feelings & Judgments about feelings & Quality of life \\
\hline Joy, Pleasure & Satisfaction & Flourishing, \\
& & Fulfilling one's potential \\
\hline & & \\
\hline
\end{tabular}

Figure 1.1. Three levels of happiness: Integrating hedonia and eudaimonia.

As an individual moves up in the levels of happiness, happiness becomes less immediate, transient, emotional, reliably measurable, and absolute, while becoming more cognitive, relative and based on norms and values.

If, in the unlikely event that positive psychology was to agree on a definition of happiness, it would constantly struggle against its multimodal common usage where happiness describes an emotional state ("I feel happy"), an overall personality style ("happy-go-lucky"), and an ultimate life goal (“all I want is to be happy") (Algoe, Fredrickson, \& Chow, 2011). However, the varying usage of happiness echoes the historical and often discordant definitions of happiness from philosophy; a discipline that has speculated about happiness long before the discipline of psychology existed.

Aristotle argued one was happy if he or she was of good birth, had good looks, wealth, and close friends - and that the happiness of man must be judged inclusive across his entire lifetime (McMahon, 2006). Aristotle used the term eudaimonia to represent happiness. The Greek term eudaimonia derives from $e u$, or "good" or "well," and daimon, which means "a spirit" or "one's personal fortune." Taken together, eudaimonia literally translates to "having a good spirit," "a good divine power," or "good fortune" (Bok, 2010). On the other hand, Epicurus believed that all of existence was experienced through the senses, including good and evil; therefore, what causes pleasure is good and pain, bad. Happiness exists where pleasure outstrips pain, known as hedonia. This form of happiness has long been the preferred understanding within the Western world. It is what utilitarianism and what people like Jeremy Bentham and John Stuart Mills strived to create. The Stoics, however, rejected the notion of pleasure and pain and instead argued that happiness was the exercise of virtue. A truly virtuous man, the Stoics 
contested, will be happy even under torture (McMahon, 2006). A contemporary philosopher also famously questioned the strict notion that happiness singularly equates to pleasure outweighing pain. Robert Nozick's (1974) thought-experiment, which was emulated in the popular movie The Matrix (Wachowski \& Wachowski, 1999), asked individuals to suppose:

There were an experience machine that could give you any experience you desired. Super-duper neuropsychologists could stimulate your brain so that you would think and feel you were writing a great novel, or making a friend, or reading an interesting book. All the time you would be floating in a tank, with electrodes attached to your brain. Should you plug into this machine for life, preprogramming your life's experiences (Nozick, 1974, p. 42)?

The resounding answer Nozick says is "no." Indeed after asking thousands of university students, on average only 5\% opt for the experience machine over reality. People seem to choose "no" because reality includes social connection, work (Diener \& Biswas-Diener, 2008), and the opportunity to make pleasure sweeter and meaning deeper through encountering and overcoming pain and challenge (a topic within positive psychology worthy of more research, see Oishi \& Kurtz, 2011).

Several researchers within the science of positive psychology, however, have called for caution: If happiness means all these different things, then the definition is too broad and it eventually means nothing as a consequence. The catch-all happiness term threatens becoming immeasurable by resisting operationalisation (Algoe, et al., 2011; Kashdan, Biswas-Diener, \& King, 2008). An answer to this problem has been to focus on the hedonic side of happiness (Kahneman, et al., 1999), and call it subjective wellbeing (SWB). Although subjective measures have often been criticized, subjective wellbeing has been shown to be easily measurable and equally valuable to understanding happiness (Diener, Sapyta, \& Suh, 1998).

This hedonic form of subjective wellbeing is popularly known as "happiness," the two terms are often interchanged throughout the psychological literature (Diener, Scollon, \& Lucas, 2009). According to Diener and colleagues, SWB is an individual's evaluation of his or her life. It is both a cognitive evaluation, an individual's level of satisfaction with their life, and emotional evaluation, i.e., how much and how intense an individual feels positive affect or the ratio of positive affect to negative affect. Although Diener stipulates that satisfaction can be either hedonic or eudaimonic (Diener, Scollon, et al., 2009), studies typically describe and use satisfaction as an indication of hedonic happiness (e.g. Extremera, Ruiz-Aranda, Pineda-Galan, \& Salguero, 2011; Sheldon \& Elliot, 1999). While this conceptualisation of subjective wellbeing has 
received a majority of the research attention, other researchers in positive psychology find that satisfaction and positive affect are not a comprehensive definition of happiness (Huta \& Ryan, 2010; Keyes \& Annas, 2009; Ryan \& Deci, 2001; Ryan \& Huta, 2009; Ryff \& Singer, 1998; Waterman, 1984, 2008; Waterman, Schwartz, \& Conti, 2008). In particular, Ryff (1989) has argued that hedonic subjective wellbeing lacked theoretical grounding and she has proposed six theoretically and empirically supported dimensions of eudaimonia: self-acceptance, positive relations with others, purpose in life, autonomy, environmental mastery, and personal growth.

Ryff and other researchers of eudaimonia argue that the full life requires both feeling good (i.e. hedonic wellbeing) and functioning well (i.e. eudaimonic wellbeing). The majority of individuals, however, report high levels of feeling good while simultaneously reporting low levels of functioning well. Anything less than high levels of both feeling good and functioning well (i.e. flourishing) is associated with greater risk of mental illness (Keyes, 2006; Keyes \& Annas, 2009). Given the importance of hedonia and eudaimonia to a positive and holistic representation of wellbeing, my thesis integrates both hedonic and eudaimonic measures of subjective wellbeing. I use this wider conceptualisation of subjective wellbeing terminology (see Keyes, Shmotkin, \& Ryff, 2002) throughout my thesis, and continue reviewing its importance in Chapter 3, to provide a complete conceptualisation of adolescent and adult wellbeing that not only questions their feeling good, but also how well they are functioning.

\section{The components of happiness}

Heritability. Individuals have little control over their long term level of happiness. In the short term, an individual can feel happy from winning the lottery; equally, in the short term, an individual can feel devastated from having the ability to walk taken from them. In the long term, however, whatever an individual's level of happiness was before their windfall or disabling accident will be where they return (Brickman, Coates, \& Janoff-Bulman, 1978). The reasoning is that people habituate and adapt to their circumstances, both positive and negative. This relatively unchangeable level of happiness is an individual's set-point, dictated by one's genes. Behavioural geneticist, David Lykken, found that thousands of identical twins raised apart maintained similar levels of happiness over time, supporting the strong role of genes and the relatively weak influence of context (Lykken, 1999, 2007; Lykken \& Tellegen, 1996). From these twin studies, Lykken and Tellegen estimated that genes account for up to $80 \%$ of an individual's level of subjective wellbeing.

However, these studies with twins, lottery winners, and paraplegic/quadriplegic respondents used hedonic wellbeing as their outcome, with no inclusion of eudaimonic 
wellbeing. Brickman and colleagues (1978) asked general questions about level of happiness and the level of pleasure derived from seven typical everyday events, while Lykken and Tellegen (1996) used the ratio between positive and negative emotionality. Appropriately, then, this theoretical position that people adapt and return to their happiness set-point regardless of context or efforts is called "hedonic adaptation." Although this position sounds fatalistic, adaptation serves an important function from an evolutionary perspective. It helps us to keep moving forward, to strive for more and to continue to better ourselves (Nettle, 2005). If people had been highly happy with the present, had not adapted, and not strived for more, we might still be travelling by horse and buggy and reading by candlelight or even, unable to read at all.

Pitfalls, however, to adaptation do exist. Let us take as an example of someone who believes that acquiring material objects will bring happiness. This person, for instance, might purchase the new Apple I-phone with the goal of feeling happier, but he or she notices that the happiness flitters away in short order. And in hopes of keeping happiness at a high level, he or she buys another new desired object and continues to repeat this cycle, striving for happiness ... this is an example of the "hedonic treadmill" (Brickman \& Campbell, 1971). The hedonic treadmill conjures an image of man in an oversized hamster wheel, repetitively chasing after things, getting those things, but remaining in the same place while exerting fruitless energy. He may sweat and work hard to keep acquiring objects—-tangible and intangible—but because he never stops to observe and attend to what he has amassed, he continues his unrewarding run. Epicurus called this dynamic, manically trying to find happiness (McMahon, 2006).

The historical knowledge that sustaining happiness is difficult appeared to influence the writers of the Declaration of Independence of the United States which only guaranteed the pursuit, not the attainment, of happiness: "We hold these truths to be self-evident: that all men are created equal, that they are endowed by their Creator with certain unalienable rights, that among these are life, liberty, and the pursuit of happiness" (Jefferson, 1776, July 4) (emphasis added by author). Pursuing happiness for its own sake, however, has been associated in war veterans with decreased hedonic and eudaimonic wellbeing and increased depressive symptoms (Kashdan, Breen, \& Julian, 2010), and in another study, even providing information about the benefits of happiness to a group of women has led to decreased happiness (Mauss, Tamir, Anderson, \& Savino, 2011). Mauss et al. argue that this phenomenon occurs because the more individuals value happiness, the more likely they will feel disappointed. These new research findings indicate that being involved in activities that are meaningful, and enhancing positive events and emotions by attending to them would be a more appropriate way to pursue (or 
stumble upon) happiness, rather than chasing the elusive emotional state of happiness. Yet, the scholars discussed previously argued that happiness is, for the most part, predetermined by genes. Why then, should anyone bother to be more aware and fully engaged with their positive experiences?

The answer is derived from recent new evidence that contests genetic determinism and the inevitability of adaptation (Boehm \& Lyubomirsky, 2009; Diener, Lucas, \& Scollon, 2006; Diener, Lucas, \& Scollon, 2009; Lyubomirsky, Sheldon, et al., 2005; Sheldon \& Lyubomirsky, 2006). These studies argue that to a certain extent, enduring levels of happiness depend on how individuals live their life, including the context in which they live and how they experience events and their circumstances. They also contest that there are several types of happiness and find that each (e.g. positive affect and satisfaction for various life domains) can have a different range (Diener, et al., 2006).

Life events. Although previous research minimized the impact of life events, recent research finds life events significantly change happiness levels for extended periods of time. Lucas (2007) summarised 21 years of longitudinal research on adaptation that he and colleagues conducted with approximately 67,000 British and German citizens from two panel studies. Lucas first criticised the comparison Brickman and colleagues (1978) made between the retrospective reports and current reports of happiness levels for patients with spinal-cord injury. Lucas highlighted that although the difference in happiness was small for these patients, there was still a difference. In fact, when the patient group was compared with the control group, there were large differences in happiness, implying major life events do matter for lasting happiness. With the longitudinal panel studies, Lucas found that changes in happiness after experiencing marriage, divorce, unemployment, and widowhood lasted anywhere from one year (for marriage) to permanently (for divorce and unemployment). The various events were also influenced by individual differences in reaction and adaptation, which Lucas attributed to variability in the nature of the event (e.g. some marriages are better than others) and to variability in individuals' reactions to similar events. Arguably, the personal variability Lucas discusses was derived from individual differences (e.g. gender, age, culture, and personality characteristics), and savoring theory would argue that the particular strategies a person uses to react to, and create, positive events may be moderated by these individual differences.

The sustainable happiness model. A newly proposed model of chronic happiness, i.e. the sustainable happiness model (Lyubomirsky, Sheldon, et al., 2005), takes into account the influence of these major life events on happiness along with genetics and activities. In total, the 
sustainable happiness model describes three factors that contribute to happiness: (1) set-point, (2) circumstances, and (3) intentional activity. The set-point contributes about 50\% to enduring happiness, a more conservative but a more widely accepted figure (Braungart, Plomin, DeFries, \& Fulker, 1992; Lucas, 2007; Tellegen, et al., 1988) than the 80\% Lykken and Tellegen (1996) stipulated. The set-point reflects the influence of temperament or personality traits, for example, negative/positive affectivity and extraversion/introversion that are highly heritable, stable across time, and centred in neurobiological systems (Davidson \& Irwin, 1999; McCrae \& Costa, 1994; Wheeler, Davidson, \& Tomarken, 1993).

New research from the field of genetics, however, is questioning the stability and rigidity of genetic expression across the lifespan. Current research in epigenetics, literally meaning "above the genome", argues that environmental factors impact genetic expression throughout the lifespan and intergenerationally (Martin, 2005; PBS, 2007). Over time, the influence of epigenes on the expression of genes grows. As the duration of environmental influences increase, the contribution of epigenes increase. The research appears to predominantly follow the development of risk and pathology (e.g. Portela \& Esteller, 2010). However, the implication for happiness research is that the accumulative contribution of the interaction between individual and environment is more important than we currently credit (Plomin \& Nesselroade, 1990). Nes (2011) concluded that high heritability is not a limitation to happiness; although genes generate stability, environments generate change and environmental influences depend on the individual. Combining the current knowledge from behavioural genetics and epigenetics, as a person agesencountering or choosing noxious or healthy substances and either negative or positive intentional activities - the way they live in their environment plays an increasingly formative role in their and their descendents' happiness.

As a general guide, the impact of circumstances, including life events, contributes an estimated 10\% to total happiness (Argyle, 1999; Diener, Suh, Lucas, \& Smith, 1999). Other circumstances include national, geographical, regional, and cultural factors, marital status, level of pay, and degree of religiosity as well as demographic characteristics such as gender and age (for a review, Diener, et al., 1999). Scholars initially hypothesised that circumstances like income would have a large effect on happiness. However, once people fulfil their basic needs, only a minor portion of happiness depends on circumstances. The robust finding that circumstantial factors only account for about $10 \%$ of the variance in happiness seemed counterintuitive. Lyubomirsky et al. (2005) suggest that this unexpectedly minor circumstantial influence on happiness is attributable to the hedonic treadmill. Adaptation occurs in situations where stimuli and the 
environment are relatively stable and consistency is a feature of most circumstantial change; the small effects are a result of people rapidly adapting to most of their circumstances (e.g.

Brickman, et al., 1978). In other words, events and circumstances on their own are largely hedonic. Getting married, receiving a raise, or getting fired are either pleasurable or unpleasurable experiences. It is when an individual reacts and attends to the circumstance with thoughts and behaviours that meaning, purpose, and gratitude can be cultivated and maintained, overcoming the dynamic of the hedonic treadmill. Although Lucas (2007) found that people often change their happiness levels for extended periods of time based on changes in their circumstances, the evidence indicates that the effect is relatively minor compared to other influences (i.e. an individual's set-point and intentional activities). Taken together, these findings show that circumstances do change individual levels of happiness, although not as greatly as heritable personality traits and the cognitive behavioural strategies people employ.

Sustainable happiness model and intentional activities. If the set-point contributes an estimated $50 \%$ to happiness and circumstances contribute an estimated $10 \%$ to happiness, then this leaves approximately $40 \%$ of happiness to be explained by intentional activities-the most fruitful route to increasing happiness (Lyubomirsky, Sheldon, et al., 2005; Sheldon \& Lyubomirsky, 2006). According to the sustainable happiness model, intentional activities are effortful discrete actions or practices that an individual can choose to engage in. The activities can be rare or habitual. Intentional activities have three specific advantages over the other two components of happiness: they are episodic, they can be varied, and they can directly counter adaptation. Choosing and implementing activities for consciously attending to everyday circumstances is one way to directly combat adaptation and the hedonic treadmill. In meditation, this could be calling attention to all the features — the look, the feel, the smell, and the taste- of simple items, for example attending to a raisin or sultana; thereby creating awareness and appreciation for the typically mundane. With savoring this might involve becoming absorbed in a common pleasant moment by slowing time, heightening particular senses, or counting one's blessings while, for instance, reading to one's child at night.

Activities can be more or less effective depending on the person-activity fit (which depends upon individual differences), the duration and frequency of the activity, if the activity is varied, and an individual's motivation and effort (Boehm \& Lyubomirsky, 2009; Lyubomirsky, 2009). When considering these moderating factors, activities like committing acts of kindness, savoring by counting blessings, writing gratitude letters, visualizing best possible futures, and savoring happy days significantly improved happiness up to six months after the active 
intervention period (Boehm \& Lyubomirsky, 2009). Preliminary work has also showed that teaching similar intentional activities to participants with mild to severe depression relieved depressive symptoms for up to one year (Seligman, Rashid, \& Parks, 2006). Impressively, positive interventions also produced higher remission rates than "treatment as usual plus medication" for outpatients with major depressive disorder. It appears that positive psychotherapy exercises ameliorate depression by both reducing negative symptoms and by directly building positive emotions, character strengths, and meaning. In sum, intentional activities support looking at individual happiness as falling within a set range rather than confining happiness to a rigid point. It is with activities like savoring that individuals will be able to feel good and function well, and more reliably achieve the top of their range.

\section{The benefits of happiness}

Scholars, authors and governmental public health policies (e.g. Fledderus, Bohlmeijer, Smit, \& Westerhof, 2010; Layard, 2006) are fervently suggesting people find motivation and devote considerable effort toward beginning and maintaining positive intentional activities, which begs the question, "Is happiness even good for you?" Are two assumptions of positive psychologists valid - that happiness is good, and that the happier one is, the better off he or she is (Oishi, Diener, \& Lucas, 2007; Oishi \& Koo, 2008)? The evidence for the first assumption is rather straight forward. Yes, happiness is good. The evidence for the second assumption is more involved.

Happiness is good. Historically positive emotions suffered neglect compared to negative emotions in psychology partly because they lacked clear definition and differentiation, and the finding that "bad is stronger than good." As a general principle, bad events, bad emotions, bad relationships and bad feedback, to name a few, have more weight on the psychology of an individual than their good counterparts (Baumeister, Bratslavsky, Finkenauer, $\&$ Vohs, 2001). An individual needs at least three daily positive emotions to every negative emotion to flourish (Fredrickson \& Losada, 2005). In marriages, five positive interactions are necessary for every single negative interaction for an enduring positive marriage (Gottman, 1994; Gottman, Coan, Carrere, \& Swanson, 1998). More positive emotions are necessary for positive daily functioning and relationships (i.e. positive experiences prevent dysfunction); however, recall that the majority of psychological research has focussed on the bad since WWII.

Fredrickson and Branigan (2001) have contributed to balancing the literature on emotion. They have discussed joy, interest, and contentment as specific examples of positive emotions that positively impact wellbeing, increasing positive physiological effects, positive 
coping, and abstract long-term thinking. Study on positive emotion and cognition has demonstrated that experienced positive affect leads to a heightened understanding and identification of the interconnection among thoughts and ideas (Isen \& Daubman, 1984), and more effective and creative thought patterns (Isen, 1987). Isen $(1999,2001)$ concluded that positive affect was positively associated with prosocial cognitions such as generosity and empathy, and prosocial behaviours such as helping. It follows that the general scientific understanding of positive emotions, now, is that they facilitate approach behaviour or continued action (for an overview, Fredrickson, 2005a).

Fredrickson (2001) recently developed a popular theory for positive emotions, called the broaden-and-build theory, whereby thought-action repertoires are expanded through cultivating positive emotions. Joy, for instance, facilitates play, creativity, and exploration, exposing an individual to novel experiences and contexts which further builds their cognitive resources. Broadening and building with positive emotions can be both an adaptive coping strategy, serving to counteract negative affect as well as increase physical, intellectual, and social resources, which in turn expand habitual modes of thinking and coping. This theory helps explain how physicians experiencing positive emotions were able to make more accurate medical diagnoses than those who were not (Isen, Rosenzweig, \& Young, 1991). It also supports the conclusion that happier people experience and react to events and circumstances in more positive and adaptive ways than unhappy people (Lyubomirsky, 2001).

Indeed the significant contribution of positive affect to physical and mental health and longevity are well documented (Cohen, Doyle, Turner, Alper, \& Skoner, 2003; Cohn, Fredrickson, Brown, Mikels, \& Conway, 2009; Diener \& Seligman, 2002; Fredrickson, Cohn, Coffey, Pek, \& Finkel, 2008; Tugade \& Fredrickson, 2004; Tugade, Fredrickson, \& Barrett, 2004; Xu \& Roberts, 2010). Two popular exemplar studies are the "nun study" and a recent metaanalysis by Lyubomirsky and colleagues. The nun study analysed the archived autobiographical journals of 180 Catholic nuns who were entering the convent at 22 years old, on average, and compared these early entries to the nuns' lifespan (Danner, Snowdon, \& Friesen, 2001). Their journals were analysed for emotional content which revealed that those nuns who expressed more positive emotional content lived healthier and longer lives over the 70 following years than those who did not. The majority of the most cheerful nuns (54\%) were still alive at the age of 94 compared with only a fraction of the least cheerful (11\%). Nuns live in virtually identical environments with similar lifestyles, surroundings, food and routine. These potentially confounding factors, for most studies, were in effect controlled by the nuns' natural 
environment, adding considerable weight to the predictive power of positive affect on longevity. The second exemplar study was a large meta-analysis of 225 published papers reviewing three classes of evidence-cross-sectional, longitudinal, and experimental-that revealed happiness, operationalised as frequent positive affect, is not only an outcome of positive life domains (e.g. health, family, work, and social) but it also leads to them over time (Lyubomirsky, King, et al., 2005). In sum, scholars have performed considerable testing and empirical validation that confirms the first assumption, that happiness is good and beneficial. I will, however, briefly continue this review of the benefits of hedonic and eudaimonic wellbeing in Chapter 3.

Happier is better. As mentioned, the second assumption is slightly more complicated. "The happier one is, the better off he or she is," depends on the definition of "better." At this stage of the argument, the definition of better may come down to individual goals and opinion. Oishi and Kurtz (2011) reviewed two longitudinal studies to discuss whether more happiness is better. In one study, researchers asked how self-rated cheerfulness upon college entry predicted three job outcomes 19 years later: current income, unemployment history, and job satisfaction (Diener, Nickerson, Lucas, \& Sandvik, 2002). In the other study, researchers asked if satisfaction at 18 years of age predicted years of higher education at age 26 , and gross income and length of intimate relationships at age 33 (Oishi, et al., 2007). The findings from these studies suggest that if earning a marginally higher annual income (approximately $\$ 500$ more) and attending more years of higher education by the time one is 26 years old is "better", then the answer is "no." More happiness does not yield these better outcomes. Rather than high cheerfulness and high satisfaction, the researchers found that the optimal level of happiness was achieved by those individuals reporting moderate cheerfulness and moderate satisfaction. It is worth noting, however, that those with the highest cheerfulness had significantly higher annual incomes (approximately $\$ 25,000$ ), more job satisfaction, and less unemployment history than the least cheerful group (Diener, et al., 2002). On the other hand, if "better" is having more long-term intimate relationships, then "yes", more happiness is better. If the goal is being at the top of social domains (e.g. time spent dating and number of close friends) versus being at the top of achievement domains (e.g. income, grades and number of missed classes) then having the highest satisfaction is the optimal level. If the goal is achievement domains, then a moderate level of satisfaction is the optimal level.

Exploring the two assumptions that happiness is good, and that the happier one is, the better off he or she is, indicates that happiness is good for physical, mental, social, work, and family health, as well as longevity. It is important to acknowledge, however, that increasing levels 
of happiness are not unequivocally best for all life domains. When the happiest group and the group just below the happiest group are compared, the relatively lower group beats the happiest group at achievement domains like income and education.

\section{Savoring is good for happiness}

Savoring may ultimately optimize both the quality and quantity of life.

(Bryant, Chadwick, \& Kluwe, 2011, p. 121)

The idea of savoring was born out of earlier work on subjective mental health (Bryant \& Veroff, 1984). Researchers concluded that a positive counterpart to coping was missing; if people make self-assessments regarding their ability to manage the stressors in life then they must also make assessments for their positive experiences. Similarly if individuals employ cognitive-behavioural approaches to regulate negative experiences, then individuals must also employ cognitive-behavioural approaches to regulate positive experiences. Theoretical models of coping (Folkman \& Lazarus, 1980) provided the initial framework for investigating the construct of savoring.

Theoretically, savoring regulates positive emotions by focussing and calling attention to a positive event or feelings about a positive event from the past (retrospection), present (appreciating), or future (anticipation) (Bryant \& Veroff, 2007). The capacity to attend to, appreciate, and enhance positive experiences is the capacity to savor (as measured by the Savoring Beliefs Inventory (Bryant, 2003). According to correlational studies, the general capacity to savor experiences is beneficial across the lifespan. The tendency to savor relates positively to subjective wellbeing in children, adolescents, university students, and the elderly (Bryant, 2003; Meehan, Durlak, \& Bryant, 1993), and is predictive of past, present, and future savoring (Bryant, 2003). This tendency to savor is also positively correlated with optimism, internal locus of control, self-control behaviours, life satisfaction and self-esteem, and negatively correlated with hopelessness, neuroticism, and depression (Bryant, 2003).

As shown in Figure 1.2, savoring is conceptualised as having three levels. The broadest level is the total experience including sensations, thoughts, behaviours, and emotions that are present while appreciating and attending to a positive stimulus, outcome or event (Bryant \& Veroff, 2007). Examples of a savoring experience include eating a gourmet meal, soaking in a hot tub after a long hike, or celebrating an accomplishment. Bryant and Veroff distinguished savoring experiences based on the primary focus of attention, as either world focussed or selffocussed (see Lambie \& Marcel, 2002). With world focussed savoring an individual is largely drawn to savor involuntarily, triggered by something or someone outside the individual (e.g. being awestruck at a museum of fine art). With self-focussed savoring, on the other hand, the 


\section{Savoring Experience}

Stimulus, outcome, event, sensations, emotions, and responses

\section{Savoring Processes}

Unfolding process: Thanksgiving, Marvelling, Basking, or Luxuriating

\section{Savoring Strategies}

Concrete thoughts and behaviours, e.g., Sharing with others and Counting blessings

Figure 1.2. Three levels of savoring.

source of positive feeling is primarily found from within the individual (e.g. pride from winning a tennis match). Bryant and Veroff also distinguished savoring experiences in terms of whether they primarily involve cognitive reflection, in which an individual introspects about their subjective experience, or experiential absorption, in which an individual favours perceptual engrossment.

The intermediate level is the savoring process: the unfolding stimulus, outcome or event that is transformed by mental and physical savoring operations into positive feelings that are also savoured (Bryant \& Veroff, 2007). Different processes are presumed to regulate different positive emotional states; for example: thanksgiving regulates gratitude, marvelling regulates awe, basking regulates pride, and luxuriating regulates physical pleasure. Although aspects of the savoring process may occur unconsciously, conscious awareness in the present moment is a prerequisite for savoring to occur.

The micro-level includes the operational components of the processes, called savoring strategies or responses. Savoring responses are specific, concrete thoughts, or behaviours that a person uses in response to, or to generate, a positive stimulus, outcome, or event. They reflect not only momentary cognitive-behavioural responses (state savoring), but also dispositional (trait) styles of savoring (Bryant \& Veroff, 2007). They are the working mechanisms within the savoring process/experience and the focus of the present research. As Bryant and Veroff commented, 
"What the fledgling field of positive psychology lacks are cogent ideas about the dynamics of positive experience, ideas about the processes that link positive events or positive personality styles with positive emotions. Without formal models of such processes, psychology lacks an understanding of the dynamics of positive feelings (p. xxiii)."

In response to this gap, Bryant and Veroff devised the Ways of Savoring Checklist (WOSC), a 60-item measure of savoring strategies that cluster together into 10 approaches (i.e. domains or factors). ${ }^{1}$ For example, within the Counting Blessings approach is the specific savoring strategy, "I thought about what a lucky person I am that so many good things have happened to me," within the Behavioural Expression approach is the specific strategy, "I jumped up and down, ran around, or showed other physical expressions of energy," and within the Absorption approach is, "I thought about the present—got absorbed in the moment.".

When describing savoring approaches and strategies, Bryant and Veroff (2007) differentiate savoring from related but separate states and processes. Mindfulness and flow (Csikszentmihalyi, 1991), for instance, share features with the sensory perceptual sharpening and absorption savoring approaches. Mindful attention, however, is open to both positive and negative stimuli, whereas savoring is attention restricted to the positive; and flow, compared with savoring, implies less conscious attention to the experience. Savoring is also related yet distinct from positive affect. Bryant and Veroff propose that savoring resulting from positive affect could be a key mechanism operating within the broaden-and-build theory of positive emotions. They believe that a person who often savors broadens his or her range of emotions and the contexts in which they can occur. In essence, positive affect can promote the savoring process. Similarly, savoring can promote the generation, continuation, and/or increase of positive affect.

Savoring: Moderator, mediator, or both? As well as being directly associated with wellbeing (e.g. Bryant, 2003; Wood, Heimpel, \& Michela, 2003), savoring is proposed to both moderate and mediate positive experience (Bryant \& Veroff, 2007). Recent research supports the assertion that meditational and moderational models do not have to be mutually exclusive, and suggest that a variable can be both a mediator and a moderator, depending on its stability (Jose \& Huntsinger, 2005). According to Baron and Kenny (1986) a moderator variable changes the strength or direction of the relationship between two variables, and a mediator variable intervenes between a variable and its outcome. As a moderator, savoring might influence the strength of the relationship from hedonia to eudaimonia, for instance. As a mediator, savoring

\footnotetext{
${ }^{1}$ See Appendix A for the 60-item WOSC.
} 
may help explain the temporal relationship between positive life events and its outcome, for example, positive affect. Although savoring is speculated to enhance the association between hedonia and eudaimonia, and increase the impact of positive events on positive emotions, I am aware of only one study under review that has investigated the possibility that savoring influences the relationship between positive life events and happiness (Jose, Lim, \& Bryant, 2011). Jose and colleagues found, using experience sampling, that daily savoring both moderated and mediated the impact of daily positive events on daily happiness. Their daily observations were, however, restricted to a university sample.

No published study of savoring strategies has included both adolescents as well as adults across the lifespan and investigated the potential moderating and mediating impact of everyday savoring strategies (recall the micro-level of savoring, Figure 1.2). To contribute to the investigation of savoring theory, my thesis examines the moderating influence of savoring on hedonia, on the one hand, and eudaimonia on the other (Chapter 3), and the mediating relationship of savoring between positive events and subjective wellbeing (Chapter 4). In Chapters 3 and 4, I continue the discussion of savoring as a moderator and mediator

\section{Savoring from adolescence to adulthood}

To contribute to the study of savoring and the field of positive psychology this thesis investigates reports of everyday positive events and everyday savoring strategies, and hedonic and eudaimonic wellbeing by a group of adolescents (13 to 15 years old) and a group of adults (16 to 88 years old).

The second half of the literature review in Chapter 2 focuses on the influences on savoring levels, including life events, age, personality, and gender. Chapter 2 aims to make three valuable contributions to the understanding of savoring. First, it aims to discover a psychometric structure of adolescent and adult everyday savoring based on an abridged version of the WOSC. An abridged form of the 60-item WOSC was developed for the present set of studies to provide a less time consuming schedule for the investigation of savoring. The entire 60-item WOSC has not appeared in any other research to my knowledge outside Bryant and Veroff's (2007) book, where it was only discussed on its own or in conjunction with a short survey. The present set of studies aims to ask several dynamic questions about the important role of savoring in wellbeing processes. By developing a shortened measure of the WOSC it was hoped that a greater understanding of the role of savoring in wellbeing, as well as the adoption of savoring in future research, would be more likely. The second aim of Chapter 2 is to investigate differences between adolescents and adults in the everyday savoring structure, and the third aim is to 
investigate differences between adolescents and adults, and between males and females for each savoring strategy. The savoring psychometric structure identified in Chapter 2 is then observed in relation to adolescent and adult wellbeing in Chapter 3. The central aims of Chapter 3 are: (1) to observe the relative ranking of adolescent and adult savoring strategies, (2) to identify which savoring strategies relate uniquely to wellbeing (i.e., satisfaction, happiness, and eudaimonia), (3) to observe the similarities and differences between the age groups for the relationships between savoring and wellbeing, and (4) to investigate the moderating property of adolescent and adult savoring on the relationship from hedonia to eudaimonia.

In the subsequent chapter, Chapter 4, I test the ability of adolescent savoring to mediate the influence of everyday positive events on their reports of hedonia and eudaimonia at two points in time over one month. Bryant and Veroff (2007) have speculated that "if a savoring process is elicited when a positive emotion is experienced, then savoring could very well be the mediating mechanism through which a person's cognitive repertoire is expanded when a positive emotion is experienced" (p. 19). Further, savoring and positive emotions are proposed to share a bi-directional relationship, an assumption that is tested along with three aims: (1) to investigate the stability and independence of savoring strategies across a month, (2) to investigate the ability of savoring to predict hedonia and eudaimonia over a month, and (3) to investigate savoring and positive affect as mediators from everyday positive events to eudaimonia.

In Chapter 5, my final empirical chapter, I include two orientations to happiness, pleasure and meaning orientations, which are analysed for their moderating influence on savoring and positive events. This chapter asks similar questions as those posed to adolescents in Chapter 4, but with adults at two points in time over three months. The aims of Chapter 5 are: (1) to confirm the stability and independence of the savoring strategies, (2) to investigate differences across young, middle, and older adults across everyday positive events and savoring, and wellbeing, (3) to investigate the direction of effects between events, savoring, and wellbeing, (4) to allow for exploration of bi-directional relationships based on implications of the structural path model, and (5) to investigate the moderating influence of orientations to happiness on savoring relationships.

The final chapter, Chapter 6, draws together the meaningful findings from the empirical chapters, Chapters 2 through 5, with a discussion of their contribution to savoring and wellbeing theories before highlighting the implications, limitations, and future directions of this research. 


\section{CHAPTER 2}

\section{The Structure of Everyday Savoring}

Major positive life events are relatively rare. We seldom go on holiday, graduate a handful of times, get married once (maybe a few more), and experience a limited number of births. Thus, it may be heartening to know that the majority of events happy people nominate make them happy are ordinary events from their everyday lives rather than special occasions (Folkman \& Moskowitz, 2000). The activities we do on an everyday basis compose a sizeable portion of our long-term happiness, about 40\%, while circumstances like major life events, status, gender, and age compose only about 10\% (Lyubomirsky, 2007; Lyubomirsky, Sheldon, et al., 2005). How do we maximise happiness, wellbeing, and satisfaction on an everyday basis? One promising way is by savoring.

This chapter explores the structure of, and similarities and differences between, adolescent and adult everyday savoring strategies. After establishing everyday savoring strategies that adolescents and adults use for responding to everyday positive events, I will test for variance in the structure of everyday savoring between these two age groups. Lastly, I will determine the influence of gender and age on everyday savoring. To date, there is no known research on everyday or adolescent savoring strategies.

\section{The Ways of Savoring Checklist}

The concept of coping with difficult events and experiences is a well-established psychological process. The coping strategies people employ depend on several factors: type of stressor, situation or context, and individual characteristics (Folkman \& Lazarus, 1980; Folkman, Lazarus, Dunkel-Schetter, DeLongis, \& Gruen, 1986; Folkman, Lazarus, Gruen, et al., 1986). The same person will respond differently to having the flu compared to experiencing the death of a friend, and another individual may respond differently to these same situations depending on their own numerous personal and contextual factors. Having certain personal characteristics and social resources during the coping process, like self-esteem and emotional support, have been found to be beneficial to health and mood (DeLongis, Folkman, \& Lazarus, 1988; Folkman \& Lazarus, 1988). Coping works by influencing the relationship between stress and emotion (Folkman \& Lazarus, 1988), and the coping strategies a person uses (e.g. problem or emotion focussed coping) impact the type (e.g. joy or anger) and intensity (e.g. temporarily sad or clinically depressed) of the emotional outcome. 
Bryant and colleagues have studied wellbeing, including coping, for over twenty years (Bryant, 1989; Bryant, Smart, \& King, 2005; Bryant \& Veroff, 1982, 1984, 2007; Jose, et al., 1998; Meehan, et al., 1993). With an in-depth understanding of the coping process, Bryant (1989) has argued that if people are capable of assessing their ability to cope with negative events, then they will likewise be capable of assessing their ability to savor positive events. By analogy, if people employ various cognitive and behavioural strategies to handle negative events and emotions, people must also employ various cognitive and behavioural strategies to enjoy positive events and emotions (Bryant \& Veroff, 2007). Similar to coping, Bryant hypothesised that particular strategies may vary based on the event, the context, and personal characteristics. Depending on the strategy used, the positive outcome may be generated, enhanced, and/or prolonged.

Strategies may also be automatic or deliberate. Adler and Fagley (2005) researched appreciation for life's events and proposed two ways that appreciation enhances wellbeing and life satisfaction: triggers or events that spontaneously and unintentionally elicit appreciation, and strategies or intentional responses people employ to increase appreciation. Witnessing a selfless act, such as a person running to the aid of someone who is injured is an example of a trigger. Purposefully saying, "thank you" to tell someone how much they are valued is an example of a strategy. As with coping strategies, savoring strategies often occur automatically and unconsciously initially and then are followed by conscious awareness often in hindsight. Savoring encompasses both spontaneous and deliberate responses with different savoring experiences (refer to Figure 1.2 and its description).

Bryant contended that there are a limited number of savoring dimensions and subsequent strategies (Bryant \& Veroff, 2007). With the goal of illuminating these particular strategies, Bryant initially asked respondents, "What was the last good thing that happened to you? Are you aware of anything you thought or did when you were experiencing this positive event that might have influenced your enjoyment of it? If so, what were those thoughts or behaviours, and how did they influence your enjoyment?” (Bryant \& Veroff, 2007, p. 88). Interestingly, between individuals, Bryant found some consistencies in the perceived effects of savoring across events and inconsistencies in the perceived effects of savoring within events. Participants reported some similar strategies for enhancing and dampening both a vacation and a date with someone special. They also reported that some of the same strategies that heightened an experience for some people dampened the experience for other people. These findings indicate that some strategies were similarly effective or ineffective across events and that particular strategies are not necessarily effective for all individuals. 
From this and further pilot data, and reviewing the literature, Bryant identified the most frequently mentioned strategies that increased, enhanced or recaptured enjoyment, and those that decreased, dampened or were ineffective for enjoyment. These items became a paper-andpencil survey with 60 close-ended questions, known as the Ways of Savoring Checklist (WOSC) (Bryant \& Veroff, 2007). The 60-item WOSC was constructed to parallel the format and design of the Ways of Coping Checklist (Folkman \& Lazarus, 1980) that asks respondents to focus on a specific stressful event and answer 66 questions about the thoughts and behaviours they employed to cope with this event. Research using the Ways of Coping Checklist has been influential across the discipline of psychology and especially in relation to clinical and health psychology (Folkman \& Lazarus, 1985b). Relative to coping, the investigation of the structure and utility of the WOSC is only just beginning, but some evidence for its role in happiness has already been documented (Diener \& Biswas-Diener, 2008; Lyubomirsky, 2007).

\section{The savoring strategies: How positive events and affect are enhanced and prolonged}

The WOSC encompasses 10 dimensions or types of savoring strategies that individually describe a variety of specific ways of thinking and behaving in response to a positive event (see Bryant \& Veroff, 2007 for a complete list of the types of savoring strategies and their respective items). The WOSC has been used with thousands of participants, in the United States, Canada, Australia and Japan, and the 10 types of savoring strategies consistently arise from the data regardless of the positive event or sample. Six of the dimensions are primarily cognitive responses (Memory Building, Self-Congratulation, Sensory-Perceptual Sharpening, Comparing, Temporal Awareness, and Counting Blessings), three use primarily behavioural strategies (Sharing With Others, Behavioural Expression and Absorption), and the last dimension also uses cognitive strategies, but works to dampen rather than enhance positive emotions (Kill-Joy Thinking). These dimensions and their reliabilities are listed in Table 2.1 in the Methods section, along with the abridged versions used for this study (pp 34-35).

The first dimension on the WOSC is Sharing With Others, which parallels social support in the coping literature, includes behaviours, e.g., "I looked for other people to share it with", and thoughts, e.g., "I thought about sharing the memory of this later with other people" (Bryant \& Veroff, 2007). Studies have found that sharing with others is associated with positive affect above and beyond the affect related to the positive event alone (Gable, Reis, Impett, \& Asher, 2004; Langston, 1994). Several mechanisms for how sharing with others-strangers and close relationships-work to improve positive affect and wellbeing above the positive event itself include increasing the perceived value of the event, and through enthusiastic responses about the 
shared good news which in turn promotes the development of trust and prosocial interaction (Reis, et al., 2010). Among adolescents, findings indicate that social support is related to positive, not negative, aspects of mental health. Social support is seen to boost adolescents' ability to obtain and savor the positive, but is perceived to be unrelated to their ability to cope with the negative (Meehan, et al., 1993). This social-behavioural dimension was the strongest predictor of level of enjoyment among North American college students when responding to a holiday or a good grade (Bryant \& Veroff).

Thinking of reminiscing about an event later or purposefully storing the images and details of a positive experience for future savoring is considered the Memory Building dimension. For example, one strategy is, "I thought about how I'd think to myself about this event later." For university students, the capacity to share with others is positively related to their ability to memory build (Bryant \& Veroff, 2007). The strong association might be that while individuals experience a positive event they are also thinking of the people they will want to share it with, but who are not present. These thoughts prompt people to actively consider the details of their positive experience in anticipation of reconstructing and sharing the joy with important relations later. The ability to build memories appears to differ between groups. Females compared to males (Bryant \& Morgan, 1986) are better able to encode positive pieces of events for later reminiscing.

A strategy that typically uses cognitive responses is Self-Congratulation or "cognitive basking", where savoring an experience takes the form of telling yourself how proud you are, or how impressed others must be. Although this is typically a cognitive strategy, Bryant points out that self-congratulation can morph into behavioural forms, like when a tennis player pumps their fist after completing an ace serve. Another cognitive strategy that can also implement behaviours is Sensory-Perceptual Sharpening. If a person is isolating or selectively enhancing attention to particular sensations, while possibly blocking others, then they are using the Sensory-Perceptual Sharpening domain. A typical example of sensory-perceptual sharpening is when someone closes their eyes to enhance the taste of delicious food or the impact of intricate music.

The Comparing dimension includes thoughts that contrast one's own feelings with others, or contrasts a situation with another from the past or imagined future. Comparing can either enhance or dampen enjoyment depending on how the contrasts are being made. If comparisons are downward, either temporally (e.g. "This is better than last time"), socially (e.g. "I'm doing better than they are"), or by using counterfactual thinking (e.g. "This experience would not have been this good if..."), then they are able to increase enjoyment. If the comparisons are upward 
contrasts (e.g. "This is not as good as the last time"), they serve to dampen enjoyment. Moreover, since comparing is thinking about what we do not have, it is better to compare the things we are happy we do not have, rather than think about the things we want, but feel we lack.

Absorption refers to holding thoughts at bay in order to become engrossed, engaged, immersed in, or inseparable from, the present moment. The aim is to not think, and instead completely become the positive experience as it develops. This process most closely resembles Csikszentmihalyi's $(1990,1997)$ optimal flow experience where a person is so involved in their activity that they lose conscious self-awareness, and a sense of self, time, and place. That is not to say that while absorbing the moment, a person loses conscious awareness. On the contrary, Lambie and Marcel (2002) argue that people are often in a state of immersion while holding a detached awareness, giving the example of having intense emotional experiences. What absorption does mean is that the enjoyment of the experience is driven by engrossment, not cognitive reflection.

The Behavioural Expression dimension is composed entirely of behavioural strategies: they are the automatic or deliberate physical expressions of feelings of joy, excitement and enthusiasm, such as laughing out loud, jumping up and down, or making verbal sounds of appreciation (e.g. humming, gasping, whistling). In contrast to sensory-perceptual sharpening, behavioural expression responses involve an acceleration of behaviours. There have been numerous studies showing that the physical expressions of happiness alone can improve mood (Kleinke, Peterson, \& Rutledge, 1998).

The Temporal Awareness dimension returns to using only cognitive strategies and refers to being thoughtful of the passage of time and wanting things to last. This process appears to be used during particularly transient and relatively rare positive events, called bittersweet moments, like graduation, birth, or marriage. A recent study of college students two weeks before graduation found that awareness of the transient nature of time increased the experience of subjective wellbeing and the engagement of appreciative behaviours, for example, walking around campus (Kurtz, 2008). Bryant contends, however, that it is not necessary to wait for fleetingly rare experiences; people, whenever desired, can deliberately employ strategies that recognise the fragility of time to benefit from this savoring approach (Bryant \& Veroff, 2007).

Counting Blessings uses primarily cognitive (e.g. "I thought about what a lucky person I am"), but also makes use of behavioural strategies (e.g. "I said a prayer of thanks"). Counting blessings, including blessings for other people and can be done inwardly and quietly, or use outward expression (e.g. saying, "Thank you” to someone). This approach creates a feeling of 
gratitude (Emmons \& McCullough, 2003; Emmons \& Shelton, 2002) and requires identifying the presence of gratitude, the source of gratitude, and the feeling of gratitude toward a source (McCullough, 2002; McCullough, et al., 2002). Using the ability to engage in counting blessings is evidenced in an increase in psychological wellbeing (Emmons \& McCullough) and is repeatedly used as a key intervention strategy in psychological research (Lyubomirsky, 2007) as well as suggested as a self-help strategy to increase happiness (Diener \& Biswas-Diener, 2008; Lyubomirsky, 2007). Although it may be difficult for some people to adopt the counting blessing strategy, especially if they do not see things that are worth being thankful for, with concerted effort gratitude can become habitual; a trait known as the grateful disposition (McCullough, et al., 2002; McCullough, et al., 2004).

Kill-Joy Thinking is the last dimension that also uses cognitive responses to positive events or emotions; however, kill-joy responses are expected to dampen enjoyment (e.g. thinking, "I didn't deserve this"). Some parallels can be drawn here to the coping literature. Where coping promotes healthy adjustment to stressors, maladaptive coping (e.g. avoidance and denial) undermines healthy adjustment; where the majority of savoring strategies enhance and prolong positive feelings, kill-joy savoring seems to hinder positive feelings.

The use of kill-joy responses appears to vary by culture. East Asian cultures endorse killjoy strategies more than European North American cultures, which may reflect Asian social norms to actively keep balance between the positive and negative (Lindberg, 2004). Level of selfesteem is also related to the use of savoring and kill-joy savoring; in the presence of positive affect, those with low self-esteem are found to use dampening techniques, while those with high self-esteem tend to use amplifying savoring techniques (Wood, Heimpel, \& Michela, 2003). At least theoretically, it appears kill-joy savoring is detrimental to wellbeing. Although there is ample extant research on the relationship between coping and adjustment, relationships between savoring strategies and positive outcomes requires empirical support.

Provided that all the items under the kill-joy dimension seem to dampen enjoyment, there are also strategies within the temporal awareness (e.g. "It would be over before I knew it") and comparing approach (e.g. "I focussed on a time when this good event would be over") that might also dampen enjoyment. It is possible that people intend to enhance positive feelings by comparing the event to others, but find they make upward (e.g. "I thought about how things might never be this good again"), rather than downward, social comparisons. Similarly, when people want to make the moment more special by thinking about its fleetingness, being temporally aware may have the unintentional affect of increasing sadness because of a focus on 
the end, rather than heightening awareness and engagement in the moment. The importance of these theoretical implications is central to the hypotheses tested in this thesis.

\section{Influences on the way people savor}

With the thousands of participants that have filled-out the WOSC, similarities and differences in the approach people use to enhance and prolong their positive emotions have become evident and are based on variations in event, personality, and gender. Some savoring strategies seem to be applied universally; others are specific to event type, personality style, or gender. The study of savoring everyday events and the influence of age on savoring strategies, however, has been a relatively unexamined area.

Events. With a sample of over a thousand university students, Bryant found that the profile for the 10 dimensions, as well as each individual dimension, varied as a function of the event experienced. Sharing, Memory Building, Sensory-Perceptual Sharpening, Comparing, Absorption, Behavioural expression, Temporal Awareness and Counting Blessings are strategies used more for a vacation than for receiving a good grade. On the other hand, students use more Kill-Joy Thinking and Self-Congratulation when receiving a good grade compared with a vacation. Regardless of event, however, it appears that particular savoring approaches are significantly inter-correlated. In response to both a vacation and good grade, Comparing, Temporal Awareness and Kill-Joy Thinking were positively, and moderately to strongly, correlated with each other. In addition, Sharing with Others moderately to strongly correlated with Memory Building, Self-Congratulation, Behavioural Expression, Absorption and Counting Blessings, and Memory Building moderately to strongly correlated with all the WOSC savoring strategies except Kill-Joy Thinking; although, Memory Building correlated with Kill-Joy Thinking moderately and positively for the good grade experience. These results indicated that the types of savoring strategies differ based on the nature of positive events or emotions that are being experienced, and that some types of savoring strategies consistently manifest themselves together across event types (Bryant \& Veroff, 2007).

With a smaller sample of undergraduate students, Bryant and Veroff (2007) investigated the recalled level and duration of enjoyment for each savoring dimension as it related to either a vacation $(N=126)$ or a good grade $(N=125)$. Particular ways of savoring (Self-Congratulations, Absorbing and Counting Blessings) were associated with stronger and longer enjoyment of both types of events. On the other hand, other ways of savoring were stronger regarding how much each event was enjoyed (Sharing, Memory Building, and Temporal Awareness were used more for a vacation, while Self-Congratulation was used more for a good grade). As Bryant 
hypothesised, all events will share, as well as differ in, elicitation of various savoring efforts. In particular, it appears that the duration of an event, for example the brief experience of receiving a good grade compared to the prolonged experience of a vacation, has a significant influence on the ways people savor.

Personality. To investigate the influence of personality variables on the way people savor, Bryant and Veroff (2007) administered the WOSC and several personality psychometric scales to measure extraversion, positive affectivity, optimism, and pessimism in 280 undergraduates (175 females). The outcome of their study indicated that those with a disposition toward positive emotions have a positive relationship with all types of savoring strategies except Kill-Joy Thinking. Extraverts used more Sharing, Memory Building, and Behavioural Expression, and the more optimistic people counted more blessings, while the more pessimistic people engaged in more Kill-Joy Thinking. Interestingly—and not hypothesised—the use of Counting Blessings and Self-Congratulation increased as the level of extraversion increased, and the level of Temporal Awareness, Sensory-Perceptual Sharpening, and Comparing increased with dispositional pessimism. It appears that extraverts prefer a wide range of strategies, from the expected outward expressions - looking to share with others and jumping up and down-to the less obvious and more reserved strategies of counting blessings and recognising one's accomplishments.

Individuals who tend to be more pessimistic rely on comparing their experience with other experiences, times, and alternative scenarios, as well as trying to block distractions and thinking that the moment is fleeting. Another, more recent study by Bryant (personal communication, March 30, 2010) found that Type A personalities reported enjoying their latest holiday less than Type B individuals. When observing types of savoring strategies, Type As used less Memory Building and Counting Blessing strategies than Type Bs. People with Type A characteristics were unable to build the enjoyable pictures and details of their holiday, or look back fondly and appreciatively as effectively as people with Type B characteristics. Overall, personality characteristics and the ways people savor are associated differently, supporting the multi-dimensional nature of savoring.

Gender. To examine possible gender differences, an independent sample of university students completed the WOSC in relation to a recent vacation ( $N=598 ; 423$ females) or good grade $(N=551 ; 421$ females). The results indicated small to moderate differences in effect size between genders. For both types of events, women reported using more Sharing, Behavioural Expression, and Counting Blessings than men, and men reported using more Kill-Joy Thinking. 
By event, women used more Memory Building in response to a vacation and Self-congratulations in response to a good grade than men. In sum, women seemed more adept at cognitive and behavioural strategies that enhance positive experiences, while men prefer strategies that interfere with enjoying positive events and correlate with pessimistic personality traits. Other research supports gender differences in savoring beliefs beginning as early as age 10 (Cafasso, Bryant, \& Jose, 1994) and last into old age (Bryant, 2003).

Age. Although gender differences in savoring appear as early as age 10, the multidimensional nature of savoring (as measured with a child version of the Savoring Beliefs Index, SBI) does not. Preadolescents display the ability to savor more globally, yet are unable to distinguish anticipatory, present, or reminiscent savoring (Cafasso, et al., 1994) as has been found with adults (Bryant, 2003) and young adults (Meehan, et al., 1993). Therefore it is argued by Bryant that savoring fully emerges in adolescence. Bryant and Veroff (2007) speculate that "the possible ways of savoring positive experience would seem to expand exponentially as adolescents enter the stage of formal operations” (p. 170). Further, cognitive development in adolescents supports their increasing ability to enjoy bittersweet events by making temporal associations (i.e. using Temporal Awareness strategies). Adolescence is a time of increasing importance for social relationships; friends and peers become ever more central to the construction of self, especially as a person moves from a dependence on family members to relating and sharing more with peers. It follows that Sharing with Others may feature strongly in adolescence as a means of increasing and prolonging enjoyment in response to positive experiences. This assumption is, in part, supported with findings from a small correlational study $(N=82)$ with 16 to 18 year olds that indicated that social support is positively associated with the ability to savor (Meehan, et al., 1993).

Although no studies have investigated the specific types of strategies adolescents use to savor positive events, a recent study on aging and emotion observed naturally occurring emotions, and the motivation to enhance, maintain, or dampen them, by 378 individuals 14 to 86 years of age (Riediger, Schmiedek, Wagner, \& Lindenberger, 2009). Compared to adults, adolescents reported wanting to dampen their positive emotions or maintain or enhance negative emotions. In contrast, adults reported wanting to maintain their positive emotions or dampen their negative emotions, more than adolescents. It appears that the negative emotionality in adolescence and the positive emotionality in older age is desired and actively maintained. Given the findings by Riediger and colleagues, I expect that the desire adolescents have to reduce their positive emotions propels them to use savoring strategies that decrease positive affect more than 
adults, while adults will use savoring strategies that maintain or increase positive affect more than adolescents.

A caveat, although differences might be found between age groups in this study and throughout this thesis, it is uncertain that these would be due to developmental influences. Potential cohort, selection and sample effects as well as methodological differences might have impacted the adolescent and adult responses. The adolescents participating in this study, for example, did not become the adults in this study. In other words, the adults' savoring might be exactly how they savored when they were adolescents and any potential differences found between the groups is attributable to cohort rather than developmental influences. In addition, the adolescents are from the Bay of Plenty region of New Zealand, while the adults are international (although the majority were from New Zealand), and the surveys between the samples were not identical. Therefore, these other potential influences on savoring and wellbeing subdues my confident interpretation of developmental differences. Given this cautionary note, finding a similar model of savoring between these two samples would reinforce the consistency and robust nature of savoring.

\section{Hypotheses: Further directions in the investigation of savoring strategies}

Previous research has supported the initial validity of the WOSC as a measure of the strategies used to enhance and prolong enjoyment from positive events. Certain savoring strategies co-occur regardless of event, while others are specific to a type of event. Individuals employ different types of savoring strategies depending on their personality, with women tending to engage in amplifying savoring while men tend to use more dampening strategies. Although differences are evident across the life-span regarding beliefs about savoring, and it may be assumed that adolescents prefer strategies that reduce positive emotions compared to adults, there is a lack of empirical research that illuminates adolescent or developmental variation in savoring strategies. Further, the WOSC is a relatively new measure and as Bryant and Veroff (2007) suggest, there needs to be continued psychometric work to support its construct validity.

The present thesis is exploring savoring in two different samples, it provides an in-depth investigation of the WOSC with three main aims: (1) to examine the structure of an abridged version of the WOSC that measures the savoring responses of both adolescents and adults to everyday positive events; (2) to investigate the similarities and differences in the composition of savoring strategies adolescents and adults employ in response to everyday positive events; and (3) to test for gender and age effects on savoring within an adolescent and an adult sample. I formed the following three research questions and related hypotheses in service of the three aims: 
(1) Is the model of savoring everyday positive events similar for adolescents and adults?

Hypothesis 1: The savoring measurement model for both age groups would have the same overall configuration.

(2) Are there differences in the multi-dimensional nature of savoring strategies between adolescents and adults?

Hypothesis 2: The adult sample would evidence a more differentiated model of savoring than the adolescent sample, congruent with previous research investigating the capacity to savor (Cafasso, et al., 1994; Meehan, et al., 1993); in other words, the relationships between savoring factors will be stronger for adolescents than for adults.

(3) Do gender and age influence savoring strategies?

Hypothesis 3: Females would endorse amplifying savoring strategies to a greater extent than males.

Hypothesis 4: Males would endorse dampening strategies to a greater extent than females, as previous research has found with holidays and good grades (Bryant \& Veroff, 2007).

Hypothesis 5: Adolescents would use more dampening savoring than adults, while adults use more amplifying savoring than adolescents.

\section{Method}

\section{Participants}

Adolescents. The adolescents were 477 school students; however, 14 students completed less than 95\% of the WOSC and were deleted from the sample (Tabachnick \& Fidell, 2001). ${ }^{2}$ The 463 remaining school students were 13 to 15 years old $(M=13.95,59 \%$ of the sample was composed of females) obtained from five schools throughout the Bay of Plenty region of New Zealand. One school was decile 8 (contributing 54 participants), one school was decile 7 (contributing 94 participants), one school was decile 4, an all girls school (contributing 72 participants), and one school was decile 2 (contributing 243 participants). ${ }^{3}$ The sample included 124 thirteen-year-olds (52 males, 72 females), 242 fourteen-year-olds (103 males, 139 females) and 97 fifteen-year-olds (33 males, 64 females). The ethnic make-up of the sample was 64\% European New Zealand/Pakeha, 34\% were Māori , .7\% were Pasifika, .9\% were Asian, 1\%

\footnotetext{
2 The 14 participants deleted were not expected to affect analyses as they comprised less than $5 \%$ of the sample (Tabachnick \& Fidell, 2001).

${ }^{3}$ School deciles are calculated using household income, occupation of parents, household crowding, educational qualifications and income support; deciles range from 1 (low Socioeconomic status) to 10 (high socioeconomic status).
} 
indicated "Other", and 14 adolescents omitted ethnicity. The frequencies were not representative of the New Zealand ethnic distribution, in that the sample included less more Māori and fewer New Zealand European, Pasifika and Asian than the most recent census, which reported 15\%, 68\%, 7\% and 9\% respectively (Statistics New Zealand, 2006). The ethnic group question allowed selection of more than one ethnic group ("tick all that apply"), which has been encouraged in social science research recently, but does pose problems for categorization, as $30 \%$ of adolescents between the ages of 10 and 14 tend to report two or more ethnicities (Kukutai, 2008). Although the study allowed adolescents to identify with multiple ethnicities, for the purposes of categorisation the present study chose only one ethnicity; when a minority ethnicity (e.g. Māori ) was selected by an adolescent along in conjunction with the majority (i.e. European New Zealand), then the minority ethnicity was prioritised for categorisation.

Adults. The adults in this study were 980 individuals ranging in age from 16 to 84 years ( $M=39,83 \%$ of the sample was comprised of females) taking part in the International Wellbeing Study. ${ }^{4}$ The sample included 258 sixteen to twenty-nine-year-olds (56 males, 202 females), 420 thirty to forty-four-year-olds (49 males, 371 females), 255 forty-five to fifty-nineyear-olds (41 males, 214 females), and 47 sixty to eighty-five-year olds (20 males, 27 females). Although the majority was from New Zealand (58\%) and the USA (30\%), the study included 34 countries. The majority was White or of European decent (83\%). Occupations included health, law, education, business professions, stay-at-home parent, undergraduate and postgraduate student, unemployed, self-employed, and public servant. The majority of adults was married $(49 \%)$, in a relationship over a year $(20 \%)$, or single (19\%). A minority of the participants was divorced $(5 \%)$, separated $(2 \%)$, in a relationship under one year $(2 \%)$, widowed $(.9 \%)$, or indicated 'other' $(.6 \%)$.

\section{Procedure}

Adolescents. The adolescent study took place approximately three months before the development of the adult study. For the adolescent study, I sent introductory letters to the principals of 12 schools in the Bay of Plenty region. A follow-up phone call was made to each principal to inquire about participation. In total, four of the 12 schools initially contacted agreed to participate. When a school wished to participate, they designated a contact person for the project; school counsellors were the contact person for three of the schools and one school

\footnotetext{
${ }^{4}$ The International Wellbeing Study (IWS) is an online 12-month longitudinal assessment study consisting of five consecutive assessment points. The project is running for four years, from March 2009 to March 2013, with rolling enrolment. The website for IWS is: www.wellbeingstudy.com
} 
nominated a deputy principal. I then made phone calls to the contacts to designate available students and facilities for the study. Electronic copies of the study's information pack (an information letter, parent consent and child assent forms) and 500 hard copies of the study questionnaires were sent to the schools. Signed parental consent and child assent forms were collected by the contact person before or at the time of data collection. Participation was confidential, voluntary, and students could withdraw at any time.

For all schools, data were collected at the school during class time. Students completed the survey in groups of 10 to 50 at locations designated by the school, for example, the scheduled classroom, a meeting hall, or the school library. In all cases a teacher, counsellor or librarian was present during data collection. I told the students that I was interested in finding out about adolescent mood, the type and impact of everyday life events, and how they thought and behaved in response to those events. Before the survey began, the survey instructions were read out to the group and students were encouraged to answer honestly and completely and to raise their hand with any questions. To adjust for low reading competency, six of the students had teacher's aides read the survey quietly and individually to them. The remaining students read the individual items themselves. On average, students took 40 minutes to complete the study questionnaire. Upon completion, students were thanked for their time, any remaining questions were answered, and students were offered a small piece of confectionary. Immediately after data collection was complete, parents and schools were sent debriefing sheets. In addition, schools ran a short informative article on the study in the school letter. On the completion of basic analyses, each school was sent a short report of the findings.

Adults. Approximately six researchers designed and lead the adult study, with several consultations with other researchers. I was consulted on which measures to include. My suggestions, however, were unable to be completely followed as several stakeholders contributed to the choice of surveys. As a result, the measures used in the adult study were often different to those in the adolescent study. A further discussion of these measures and their differences as they pertain to this study will be discussed in the measures section that follows.

The adult study was advertised through newsletters, email lists, website postings, and texting companies. Participants registered for the study online via the International Wellbeing Study website and were subsequently alerted by email when the study opened. If participants failed to complete the online survey three weeks after notification, they were sent a second email. If the survey was not completed during the open assessment month (March, 2009), individuals were omitted from the study. Incentives for participation included individualised assessment 
reports provided after a year of participation, draws for $15 \$ 100$ Amazon.com vouchers, and the opportunity to participate in one of two brief internet-based courses.

\section{Measures}

The adolescent and adult surveys included scales measuring retrospective reports of the intensity of everyday positive events, savoring strategies, life satisfaction, wellbeing, and happiness over the previous month in the case of adolescents, and the last three months in the case of adults. Of note, and as will be noted throughout this thesis, the use of different measures between the adolescent and adult studies attenuates the interpretation of developmental influences on savoring and its relationship to wellbeing. The everyday Positive Life Events scale, for example, asked about more concrete events for adolescents and more general events for adults which could influence differences in savoring responses between the groups.

Everyday Positive Life Events. The everyday Positive Life Events scale (PLE) was designed by Jose, patterned after Jose and colleagues' previous measure of Everyday Negative Life Events (Jose, Cafasso, \& D'Anna, 1994; Jose, et al., 1998). The PLE was used to measure adolescents' perceived intensity of 25 everyday positive events. ${ }^{5}$ Participants were first asked whether these experiences had happened over the last month (adolescents) or last three months (adults), and responded with either 'yes' or 'no' (e.g. 'someone complimented you'). The frequency question was then followed with the intensity question, 'If you said 'yes', how much of a positive experience was it?' $(0=$ none; $1=$ a little; $2=$ some; $3=$ a lot $)$. Exploratory Factor Analysis using SPSS indicated a one-factor model, with high internal reliability, $\alpha=.85$. Adults reported on an abridged 5-item form of the PLE with an internal reliability of $\alpha=.72 .{ }^{6}$ Immediately after filling out the PLE, both samples were asked how they responded to (savored) their everyday positive events.

Savoring. As described in Chapter 1, the 60-item Ways of Savoring Checklist (WOSC) is a relatively time consuming questionnaire and has been in limited use since its release in 2007. I developed an abridged savoring scale to shorten completion time, limit fatigue, and to help facilitate additional variables being examined in conjunction with savoring. Previous tests of readability revealed that the WOSC items were at the reading level appropriate for a 9-year-old (Bryant \& Veroff, 2007). To support the structure of the original WOSC, I selected items from each of the 10 WOSC subscales. The first three items with the least number of words from each

\footnotetext{
${ }^{5}$ See Appendix B for the adolescent PLE scale.

${ }^{6}$ See Appendix $C$ for the adult PLE scale.
} 
of the 10 subscales were chosen for the adolescent study. ${ }^{78}$ The collaborators of the adult study reduced this abridged WOSC further, to 20-items, by removing the last 10 items from the questionnaire. ${ }^{9}$ To affect continuity and modelling, the adolescent 30 -item version was further reduced to mirror the 20 -item adult version. The reliabilities and number of items for the 10 subscales of the original 60-item WOSC and the abridged 20 -item adolescent and adult versions of these subscales are listed in Table 2.1.

Table 2.1. Cronbach's Alpha and Number of Items for each Dimension of the Original 60-item WOSC for Young Adults, and the 20-item abridged WOSC used with Adolescents and Adults.

\begin{tabular}{|c|c|c|c|c|}
\hline & \multirow[b]{2}{*}{ Dimension } & \multicolumn{3}{|c|}{ WOSC Measures and Sample Type } \\
\hline & & $\begin{array}{l}\text { 60-item Original } \\
\text { Young Adult }\end{array}$ & $\begin{array}{l}\text { 20-item Abridged } \\
\text { Adolescent Study }\end{array}$ & $\begin{array}{l}\text { 20-item Abridged } \\
\text { Adult Study }\end{array}$ \\
\hline 1. & Sharing With Others & .86 (6 items) & .46 (2 items) & .61 (2 items) \\
\hline 2. & Memory Building & .89 (7 items) & .46 (2 items) & .47 (2 items) \\
\hline 3. & Self-Congratulation & .84 (7 items) & .64 (2 items) & .53 (2 items) \\
\hline 4. & Comparing & .78 (7 items) & .33 (2 items) & .28 (2 items) \\
\hline 5. & $\begin{array}{l}\text { Sensory-Perceptual } \\
\text { Sharpening }\end{array}$ & .73 (4 items) & .53 (2 items) & .44 (2 items) \\
\hline 6. & Absorption & .74 (4 items) & .37 (2 items) & .15 (2 items) \\
\hline 7. & Behavioural Expression & .82 (6 items) & .60 (2 items) & .64 (2 items) \\
\hline 8. & Temporal Awareness & .82 (5 items) & .45 (2 items) & .62 (2 items) \\
\hline 9. & Counting Blessings & .72 (3 items) & .68 (2 items) & .75 (2 items) \\
\hline 10. & Kill-Joy Thinking & .80 (7 items) & .28 (2 items) & .40 (2 items) \\
\hline
\end{tabular}

Note. The reliability coefficients for the original 60-item WOSC dimensions were derived from a survey with 1,136 young adults (undergraduate university students) who were instructed to think and write about a recent positive event and then fillout the WOSC by Bryant (Bryant \& Veroff, 2007). The 20-item WOSC adolescent version was used with 463 adolescents between the ages of 13 to 15 who first reported on a set of 25 positive life events and were then asked to fill-out the WOSC in response to these. The 20 -item WOSC was used with 980 international adults, 16 to 85 years old, who first replied to a set of 5 positive life events and were then asked to fill-out the WOSC in response to these.

Participants responded on a 7-point Likert scale $(1=$ strongly disagree; $4=$ neutral; $7=$ strongly agree) indicating how they responded to positive events (listed just before in the PLE measure) during the past month (adolescents) or three months (adults). The overall internal

\footnotetext{
7 See Appendix D for the adolescent abridged WOSC.

${ }^{8}$ The same 30 items were found to be the highest loading items for their represented subscales (Jose et al, 2012).

${ }^{9}$ See Appendix E for the adult abridged WOSC.
} 
reliabilities of the abridged 20-item WOSC for adolescents and adults was high, $\alpha=.87$ and $\alpha=$ .82 respectively. Table 2.2 is a list of the 20 items for the adolescent and adult samples.

Table 2.2. The 20 Items from the Abridged W ays of Savoring Checklist.

\section{Constituent Abridged WOSC items}

1. I thought about sharing the memory of this later with other people. (SWO)

I tried to take in every sensory property of

2. the event (sights, sounds, smells etc.). (MB)

I reminded myself how transient this

3. moment was — thought about it ending. (TA)

I jumped up and down, ran around, or

4. showed other physical expressions of energy. (BE)

I thought back to events that led up to it-

5. to a time when I didn't have it and wanted it. (Comp)

6. I thought only about the present-got absorbed in the moment. (Abs)

7. I reminded myself how lucky I was to have this good thing happen to me. (CB)

8. I told myself why I didn't deserve this good thing. (KJ)

9. I looked for other people to share it with. (SWO)

10. I thought about how I'd think to myself about this event later. (MB)
11. I laughed or giggled. (BE)

12. I opened my eyes wide and took a deep breath-tried to become more alert. (SPS)

13. I closed my eyes, relaxed, took in the moment. (Abs)

I thought about what a lucky person I am

14. that so many good things have happened to me. (CB)

15. I thought about ways in which it could have been better. (KJ)

16. I told myself how proud I was. (SC)

17. I reminded myself that it would be over before I knew it. (TA)

18. I focussed on the future-on a time when this good event would be over. (Comp) I tried to slow down and move more

19. slowly (in an effort to stop or slow time). (SPS)

20. I told myself how impressed others must be. (SC)

\section{Results}

\section{Analytic Strategy}

The first goal was to assess whether adolescents and adults used a similar model of savoring strategies using statistical equation modelling (SEM) in AMOS (Arbuckle, 2009). Prior to conducting analyses, a process of pre-operational explication (Cook \& Campbell, 1979) was extended from Bryant and Veroff (2007). To derive a theoretically robust measurement model four guiding principles were used for constructing a meaningful measure of everyday savoring: (1) savoring strategies are processes rather than states or outcomes; (2) understanding the 
relationship of everyday savoring at the item-level will provide a meaningful structure of everyday savoring (Little, Cunningham, Shahar, \& Widaman, 2002); (3) items would be grouped into factors based on common themes (e.g. cognitive versus behavioural, calm strategies versus more active strategies, and dampening versus amplifying strategies); and (4) a measure of everyday savoring would feature strategies that respond to transient positive everyday events (e.g. receiving a compliment and then Sharing it with Others) over those that accompany prolonged positive events (e.g. Memory Building by taking mental photographs of a beautiful holiday).

A recent review of emotion regulation research (Koole, 2009) has highlighted the rare but necessary use of both top-down and bottom-up approaches to understanding emotion regulation strategies; therefore, both a theoretical and empirical approach (i.e. confirmatory factor analysis) were applied to the structural analyses of everyday savoring . Several theoretical models were formed, from the most parsimonious to the 10-factor multi-dimensional structure that has been extensively supported by Bryant's research. Then the models were submitted to confirmatory factor analyses (CFA) using AMOS 18 (Arbuckle, 2009)

Following the method outlined by Byrne (2001) for testing the factorial validity of a theoretical construct, CFA was employed as it rigorously tests an a posteriori factor structure using a variety of fit indices (Bryant \& Yarnold, 1995). However, difficulty with over-identifying models due to too many items per factor or under-identifying models due to too few items per factor has been an issue for SEM; 3 to 4 items per factor has been suggested as the optimal ratio (Kline, 2005). This implies that the original 10-factor structure of the WOSC found by Bryant and Veroff (2007) will not be supported here, as the present study has only 2 items to identify each of the 10 factors. The 10 factor model, however, will still be tested, but it is important to note limitation of the chosen analysis with the present data. Once models were established, confirmatory factor analysis was used in a post-hoc capacity for model refinement (Byrne).

After the most acceptable models were refined, the second goal, namely, of testing model differences between age samples, was investigated. The accepted models for adolescent and adult savoring strategies were combined to form a baseline model and tested for invariant factorial structure of a theoretical construct using the method outlined by Byrne (2001). This procedure involves a process of constraining parameters in a sequential order to observe possible significant differences in the chi square statistic using AMOS (Arbuckle, 2009). Significant differences indicate a difference in the model between groups. First, the equality of the model is tested with an initial omnibus test to see if the measurement model functions equivalently across the groups. If equality is not supported, further tests are performed, beginning with investigating 
factor loadings (testing metric invariance) and ending with factor variances and covariances (testing configural invariance). During this process, any non-significant constraints are left constrained, while significantly different parameters between the groups are released to be freely estimated.

To achieve the third goal, multivariate analyses for each sample were conducted to examine the effects of gender and age on everyday savoring. In cases of significant multivariate results, univariate analyses of variance were then conducted. When univariate effects were found for gender, focussed $t$-tests were used to isolate the occurrence of gender differences across age, and effect sizes were calculated.

\section{Factorial validity/configural analysis: Is the model of savoring everyday positive events similar for adolescents and adults?}

The first aim of the study was to investigate the similarities and differences in the composition of savoring strategies adolescents and adults employ in response to everyday positive events. Assumptions of testing factorial validity with the maximum likelihood (ML) method employed by AMOS are that the sample is very large, the distribution of observed variables is multivariate normal, the hypothesised model is valid (West, Finch, \& Curran, 1995), and the form of observed variables is continuous. The range for recommended sample size to number of estimated parameters is at least 5-10:1 for configural analyses (Kline, 2005). The original 10 factor solution used 115 estimated parameters that resulted in a ratio of 4:1 with the adolescent sample (considered to be slightly below acceptable), however, the adult sample had a ratio of 9:1 and both models displayed similar model fit indices. For all other models tested, the ratio was within the recommended range for both samples.

The data were minimally non-normally distributed. No outliers in the adolescent sample existed, while the adult sample had a total of $26 .{ }^{10}$ Univariate skewness (SK) values for the adolescent sample ranged from -.01 to .71, with a mean SK of .42; univariate kurtosis (KU) ranged from -1.05 to .03, with a mean $\mathrm{KU}$ of .66. Univariate skewness (SK) values for the adult sample ranged from -1.55 to .77 , with a mean SK of .67; univariate kurtosis (KU) ranged from 1.22 to 2.29 , with a mean $\mathrm{KU}$ of .90 . Although there is no acceptable degree of non-normality, studies that have evaluated the impact of non-normality on ML analyses found that problems

\footnotetext{
10 The outliers for the adult sample were: four respondents who answered Strongly disagree to item 1; four respondents who answered Strongly disagree to item 7; four respondents who answered Strongly agree to item 9; three, four, and three respondents who answered Strongly disagree, Disagree, and Slightly disagree, respectively, to item 9; and four respondents who answered Strongly disagree to item 11. Two of the 26 outlying responses were made by the same respondent. Table 1.2 displays items.
} 
may occur when values of SK approach 2 and KU approach 7 (Chou \& Bentler, 1995; Curran, West, \& Finch, 1996). As the SK and KU values in the present study fell below the level considered to be problematic, and since transformation of variables is not universally recommended due to the argument that transformed variables can be harder to interpret (Tabachnick \& Fidell, 2007), non-transformed items were subjected to modelling. ${ }^{11}$ The last two assumptions of ML analyses are that the model is valid and data are continuous in form, and both of these were met by using a pre-operational explication process and by employing a number of Likert scale-based items with large sample sizes.

The 20 WOSC items that were common between the adolescent sample of 463 and the adult sample of 980 were used for the confirmatory maximum likelihood factor analyses of the savoring model (see Table 2.2). The validity of several models was tested for the adolescent and adult samples, from a one-factor parsimonious structure to the ten-factor model derived by Bryant in numerous studies with university undergraduates (Bryant \& Veroff, 2007).

The overall model fit statistic is the chi square $\left(\chi^{2}\right)$ or likelihood ratio test, however it is widely known to be affected by sample size (Kline, 2005). To decrease the $\chi^{2}$ sensitivity, it can be divided by the degrees of freedom (df). This ratio generally results in a lower value called the normed chi squared $\left(\chi^{2} / \mathrm{df}\right)$, however, there is no definitive cut-off, with values below 3.0 and 5.0 being mentioned as acceptable (Bollen, 1989). Because of the difficulties that face the $\chi^{2}$ statistic, evaluating a combination of test statistics has been recommended (Byrne, 2001; Kline, 2005).

The goodness of fit for each model was assessed using four criteria (Hu \& Bentler, 1999): the $\chi^{2} / \mathrm{df}$ ratio, the standardized version of Jöreskog and Sörbom's (1982) root mean square residual (sRMR), root mean square error of approximation (RMSEA) (Steiger, 1990), and the comparative fit index (CFI) (Bentler, 1990). Absolute fit was represented by sRMR and RMSEA, and incremental fit by CFI. ${ }^{12}$ For sRMR and RMSEA, smaller values reflect better model fit; for CFI, larger values reflect better model fit. Hu and Bentler suggest cut-off values of .08 for sRMR, .06 for RMSEA and .95 for CFI, with Bentler and Bonnett (1980) recommending CFI values above .90. All models and their goodness of fit are reported in Table 2.3.

\footnotetext{
${ }_{11}$ As a heuristic, several models were also tested with transformed variables with no reportable variance in model fit. In addition, models were subjected to bootstrapping in AMOS, where covariance biases (the difference in ML and bootsrap covariances) were low, ranging from -.013 to .006 with an average of .006.

${ }^{12} \mathrm{Hu}$ and Bentler (1999) discuss finding that sRMR was the most sensitive index to misspecified factor covariances or latent structures, and RMSEA and CFI were among the most sensitive indices to models with misspecified factor loadings.
} 
Table 2.3. Goodness-of-fit Statistics for CFA Measurement Models of the 20-item WOSC in Response to Everyday Events, Adolescent $(N=463)$ and Adult $(N=980)$ Samples.

\begin{tabular}{|c|c|c|c|c|c|c|c|}
\hline & \multirow[b]{2}{*}{ CFA Model } & \multirow[b]{2}{*}{ Sample } & \multicolumn{5}{|c|}{ Measure of Model Fit } \\
\hline & & & $\chi^{2}$ & $\mathrm{df}$ & sRMR & CFI & RMSEA \\
\hline \multirow{2}{*}{1.} & 1 Global Factor (all & Adolescent & 1803.51 & 405 & .08 & .69 & .09 \\
\hline & (WOSC Total) & Adult & 2356.96 & 170 & .11 & .58 & .12 \\
\hline \multirow{2}{*}{2.} & 10 Factors & Adolescent & 1196.3 & 360 & .07 & .81 & .07 \\
\hline & (Original Factors) & Adult & 921.07 & 125 & .08 & .85 & .08 \\
\hline \multirow{2}{*}{3.} & 2 Factors & Adolescent & 47.03 & 13 & .05 & .93 & .08 \\
\hline & (Dampen, Amplify) & Adult & 81.55 & 13 & .06 & .93 & .07 \\
\hline \multirow{2}{*}{4.} & 2 Factors & Adolescent & 39.85 & 13 & .04 & .96 & .07 \\
\hline & $(\mathrm{HA}, \mathrm{SF})$ & Adult & 99.03 & 13 & .05 & .95 & .08 \\
\hline \multirow{2}{*}{5.} & 3 Factors & Adolescent & 86.44 & 32 & .04 & .94 & .06 \\
\hline & (LA, HA, SF) & Adult & 153.79 & 32 & .04 & .94 & .06 \\
\hline \multirow{2}{*}{6.} & Model 5 with factor & Adolescent & 116.89 & 35 & .07 & .91 & .07 \\
\hline & constrained & Adult & 214.36 & 35 & .07 & .91 & .07 \\
\hline \multirow{2}{*}{7.} & 4 Factors & Adolescent & 161.33 & 59 & .05 & .92 & .06 \\
\hline & (DS, LA, HA, SF) & Adult & 369.20 & 59 & .06 & .89 & .07 \\
\hline \multirow{2}{*}{8.} & \multirow{2}{*}{$\begin{array}{l}\text { Model } 7 \text { with post- } \\
\text { hoc cross-loading }\end{array}$} & Adolescent & 147.83 & 58 & .04 & .93 & .06 \\
\hline & & Adult & 237.11 & 57 & .04 & .94 & .06 \\
\hline
\end{tabular}

Models 1 and 2: Parsimonious to complex. The simplest model (Model 1) specified one global savoring factor and assumed that savoring was unidimensional; namely, that people only vary in the degree to which they use savoring. To test the fit of this one-factor model, all 20 WOSC items were constrained to load on the same factor. On the other extreme, the most complex 10-factor model of the WOSC (Model 2) assumed that savoring strategies had 10 dimensions; namely, that people savor everyday events in 10 unique ways. To test the fit of this model, the corresponding two items were constrained to their respective 10 factors. 
Models 3 and 4: Two factors. A two-factor structure was also modelled, based on the assumption that people either positively savor (i.e. amplify) or negatively savor (i.e. dampen). Two dimensions were modelled, one constrained positive items (items were: 1) Sharing with Others; 4) Behavioural Expression; 7) Counting Blessings; 16) Self-congratulations), and one constrained negative (or dampening) items (items were: 15) Kill-joy; 17) Temporal Awareness; 18) Comparing) (and this two factor model was named Model 3). To obtain the best two-factor fit, Memory Building was not included. For this model, it was assumed that Memory Building was not particularly useful for everyday events. In addition, no items representing low arousal strategies like Absorption and Sensory Perceptual Sharpening were included because they have been linked to longer duration events than were queried in this study (Bryant \& Veroff, 2007).

A second two-factor model (Model 4) assumed people use either active/outward forms of savoring or cognitive self-reflective savoring for everyday positive events. This model omitted negative and low arousal strategies, and instead constrained a two dimensional model of High Arousal savoring (items were: 1) and 9) Sharing with Others; 4) and 11) Behavioural Expression), and Self-focus savoring (items were: 7) Counting Blessings; 10) Memory Building; 16) Selfcongratulations).

Models 5 and 6: Three factors. The three factor alternative model specified that people use savoring strategies along three dimensions by responding to positive everyday events using quiet, low arousal forms of savoring, expressive/active forms of savoring, and selffocused/reflective forms of savoring. A three factor model was tested, encompassing the previously omitted Low Arousal items (items were: 12) and 19) Sensory Perceptual Sharpening; 13) Absorption) as well as High Arousal and Self-focus strategies; this three factor model was named Model 5. To investigate the possibility that a simpler explanation of amplifying savoring could be supported, the three amplifying factors were constrained to test whether a more parsimonious structure would better explain amplifying savoring (and this return to a one factor model was named Model 6); in other words, the factor covariances were constrained and the model fit indices between Model 5 and Model 6 were compared. The contrast of the nested model ensured that the multidimensional construct was a significant improvement in goodnessof-fit over the one-factor construct of positive savoring for both the adolescent and adult samples, with a likelihood ratio test, $p<.001$.

Models 7 and 8: Four factors. The final model assumed people savor along four dimensions. This model used the previous three factor model and also included a cluster of dampening savoring strategies, testing the three positive savoring factors (Low Arousal, High 
Arousal, and Self-focus savoring) as well as including the use of the Dampening savoring from Model 3 (and this four factor model was named Model 7). Two post-hoc refinements were made to improve the model (Byrne, 2001). For adolescents and adults, Sensory Perceptual Sharpening (item 19) was allowed to load onto the Dampening factor. For adults, Memory building was allowed to load onto the Dampening factor (item 10) (and this variant of the four factor model was Model 8). The items and internal reliability of each factor are represented in Table 2.4.

Table 2.4. Factors, Cronbach's Alphas and Items for the Four-Factor Model of the Abridged WOSC in Response to Everyday Events.

\begin{tabular}{|c|c|c|c|c|}
\hline Factor & Adolescent & Adult & & Abridged WOSC items \\
\hline \multirow{3}{*}{$\begin{array}{l}\text { Dampening } \\
\text { Strategies }\end{array}$} & \multirow{3}{*}{.62} & \multirow{3}{*}{.69} & 15. & $\begin{array}{l}\text { I thought about ways in which it could have been } \\
\text { better. }(\mathrm{KJ})\end{array}$ \\
\hline & & & 17. & $\begin{array}{l}\text { I reminded myself that it would be over before I } \\
\text { knew it. (TA) }\end{array}$ \\
\hline & & & 18. & $\begin{array}{l}\text { I focussed on the future-on a time when this } \\
\text { good event would be over. (Comp) }\end{array}$ \\
\hline \multirow{3}{*}{$\begin{array}{l}\text { Low Arousal } \\
\text { Strategies }\end{array}$} & \multirow{3}{*}{.65} & \multirow{3}{*}{.61} & 12. & $\begin{array}{l}\text { I opened my eyes wide and took a deep breath- } \\
\text { tried to become more alert. (SPS) }\end{array}$ \\
\hline & & & 13. & $\begin{array}{l}\text { I closed my eyes, relaxed, took in the moment. } \\
\text { (Abs) }\end{array}$ \\
\hline & & & 19. & $\begin{array}{l}\text { I tried to slow down and move more slowly (in an } \\
\text { effort to stop or slow time). (SPS) } *\end{array}$ \\
\hline \multirow{4}{*}{$\begin{array}{l}\text { High } \\
\text { Arousal } \\
\text { Strategies }\end{array}$} & \multirow{4}{*}{.66} & \multirow{4}{*}{.70} & & $\begin{array}{l}\text { I thought about sharing the memory of this later } \\
\text { with other people. (SWO) }\end{array}$ \\
\hline & & & 4. & $\begin{array}{l}\text { I jumped up and down, ran around, or showed } \\
\text { other physical expressions of energy. (BE) }\end{array}$ \\
\hline & & & 9. & I looked for other people to share it with. (SWO) \\
\hline & & & 11. & I laughed or giggled. (BE) \\
\hline \multirow{3}{*}{$\begin{array}{l}\text { Self-focus } \\
\text { Strategies }\end{array}$} & \multirow{3}{*}{.60} & \multirow{3}{*}{.55} & 7. & $\begin{array}{l}\text { I reminded myself how lucky I was to have this } \\
\text { good thing happen to me. (CB) }\end{array}$ \\
\hline & & & 10. & $\begin{array}{l}\text { I thought about how I'd think to myself about this } \\
\text { event later. }(M B)^{* *}\end{array}$ \\
\hline & & & 16. & I told myself how proud I was. (SC) \\
\hline
\end{tabular}

Note. ${ }^{*}$ Cross-loads onto Dampening Strategies for both the Adolescent Sample $(\mathrm{N}=463)$ and Adult Sample $(\mathrm{N}=$ 980). ${ }^{* *}$ Cross-loads onto Dampening Strategies for the Adult Sample only.

A perusal of Table 2.3 indicates that the obtained model fit indices of the eight models supported the refined four-factor measurement model (Model 8, containing the four factors of Dampening, Low Arousal, High Arousal, and Self-focused) as an acceptable representation of 
savoring strategies for both adolescents and adults..$^{13}$ The four factor model included the highest number of representative savoring items from each of the 10 factors on the original 60 -item WOSC, other than the unacceptable one factor and ten factor models. By all four statistical criteria, the four factor model with post-hoc refinement was supported by an acceptable goodness-of-fit to the WOSC data, see Figure 2.1.

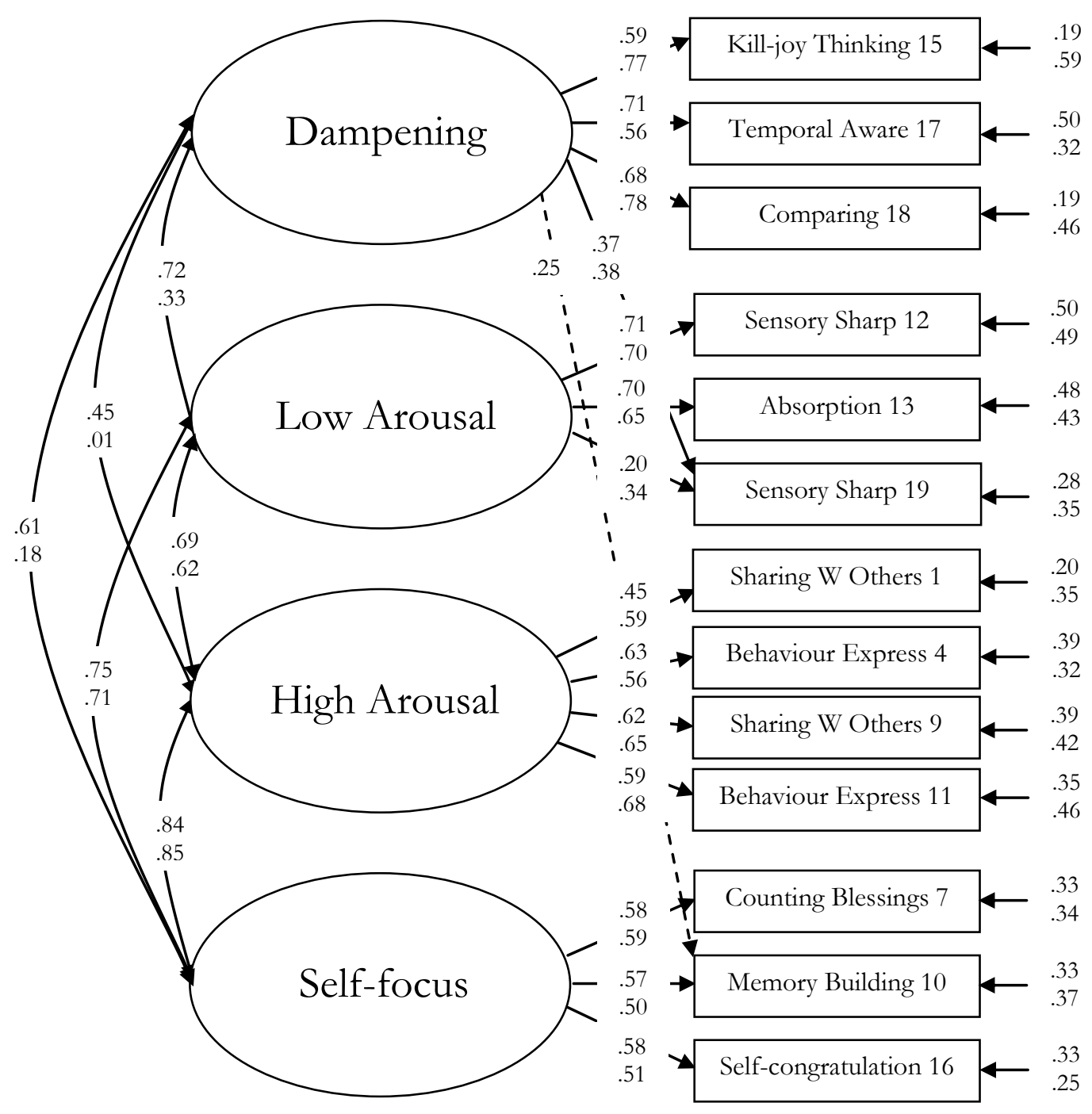

Figure 2.1. Four-factor model of savoring strategies (standardised estimates). Note. Top values = adolescents; bottom values $=$ adults. The dashed arrow indicates a post-hoc relationship for adults only.

${ }^{13}$ Further, the four-factor model was confirmed with a subsample of the adults, where females were randomly selected to equal the male sample size. In addition, the four-factor model was confirmed with a subsample of the adults, where adults were only selected if from New Zealand. 
Both the adolescent $\left(\chi^{2} / \mathrm{df}=2.55, \mathrm{CFI}=.93, \mathrm{RMSEA}=.06, \mathrm{sRMR}=.04\right)$ and adult $\left(\chi^{2} / \mathrm{df}=4.16, \mathrm{CFI}=.94, \mathrm{RMSEA}=.06, \mathrm{sRMR}=.04\right)$ samples met the criteria for sRMR and RMSEA cut-off (i.e. they were at or below .08 and .06 respectively), and were above the recommended criteria of $90 \mathrm{CFI}$. The four factor model with post-hoc refinement also explained a similar sizeable proportion of the variance in individual WOSC items for both the adolescent (overall mean $R^{2}=.37$ ) and adult (overall mean $R^{2}=.38$ ) groups. In addition, all items loaded significantly on their respective latent variables with standardised regression weights between .20 and .71 , with an average of .60 for adolescents, and between .25 and .78 , with an average of .66 for adults (Table 2.4). As seen in Figure 2.1, the correlations for latent savoring variables were moderate to high for adolescent (from .45 to .84 ) and low to high for adults (from -.01 to .85$)$.

Model 5 (Table 2.3) was the only other model to provide a good fit to the data for both adolescents and adults. Although this three factor model (Model 5, Table 2.3) had an apparently equally good fit to the data as the four factor model that was chosen here (Model 8, Table 2.3), the three factor model had six fewer items and lacked the dampening factor. Similar to the importance of understanding maladaptive coping strategies and its impact on individual adjustment within the wider scope of coping, it is important to understand dampening savoring strategies within the wider scope of savoring. In addition, dampening savoring may influence wellbeing differently (e.g. positively, neutrally, or negatively) depending on the culture of the individual (Lindberg, 2004), or in the case of the present study, depending on age.

Taken together, these findings suggest that adolescents and adults make similar distinctions in their ways of savoring everyday positive events in terms of Dampening, High Arousal, Low Arousal, and Self-focus savoring, supporting the first hypothesis that a savoring measurement model for both age groups would have the same overall configuration. The result was a four factor model of savoring for both adolescents and adults that modelled 13 individual strategies, with at least one item from each of the original 10 WOSC factors.

\section{Structural Invariance: Are there differences in the multi-dimensional nature of savoring strategies between adolescents and adults?}

Recall that the second aim of the study, given adequate factorial validity, was to investigate the differences in the factor structure for the savoring model between adolescents and adults. A sequential procedure of constraining parameters and observing significant changes in the chi square value and degrees of freedom from the baseline model was used to test Hypothesis 2. Recall from the Analytic Strategy that a significant difference between a 
constrained model and the baseline model (i.e. the combined adolescent and adult model where all parameters are freely estimated) indicates a violation of the assumption of invariance or, in this case, that relationship(s) within the structure of savoring are significantly different across adolescents and adults.

The chi square value, degrees of freedom, change in chi square value, change in chi square, and level of significance (i.e. $p$ value) for each model is listed in Table 2.5. The adolescent

Table 2.5. Goodness-of-Fit statistics for Tests of Invariance across Adolescent $(\mathrm{N}=463)$ and Adult Samples (N = 980): A summary.

\begin{tabular}{|c|c|c|c|c|c|c|}
\hline & Model Description & $\chi^{2}$ & df & $\Delta \chi^{2}$ & $\Delta \mathrm{df}$ & $p$ value \\
\hline 1 & $\begin{array}{l}\text { Combined baseline models (Adolescent } \\
\text { and Adult) }\end{array}$ & 384.97 & 114 & - & - & - \\
\hline 2 & $\begin{array}{l}\text { Factor loadings, variances, and } \\
\text { covariances constrained equal }\end{array}$ & 456.00 & 134 & 71.03 & 20 & $p<.001$ \\
\hline 3 & Factor loadings constrained equal & 399.46 & 124 & 14.49 & 10 & NS \\
\hline 4 & $\begin{array}{l}\text { Model } 3 \text { with all variances constrained } \\
\text { equal }\end{array}$ & 402.76 & 128 & 17.75 & 14 & NS \\
\hline 5 & $\begin{array}{l}\text { Model } 4 \text { with all covariances constrained } \\
\text { equal }\end{array}$ & 480.18 & 135 & 95.21 & 21 & $p<.001$ \\
\hline 6 & $\begin{array}{l}\text { Model } 4 \text { with covariance between } \\
\text { Dampening/Low Arousal constrained } \\
\text { equal }\end{array}$ & 430.68 & 129 & 45.71 & 15 & $p<.001$ \\
\hline 7 & $\begin{array}{l}\text { Model } 4 \text { with covariance between } \\
\text { Dampening/High Arousal constrained } \\
\text { equal }\end{array}$ & 436.24 & 129 & 51.27 & 15 & $p<.001$ \\
\hline 8 & $\begin{array}{l}\text { Model } 4 \text { with covariance between } \\
\text { Dampening/Self-fucus constrained } \\
\text { equal }\end{array}$ & 429.10 & 129 & 44.13 & 15 & $p<.001$ \\
\hline 9 & $\begin{array}{l}\text { Model } 4 \text { with covariance between } \\
\text { Low/High Arousal constrained equal }\end{array}$ & 402.80 & 129 & 17.83 & 15 & NS \\
\hline 10 & $\begin{array}{l}\text { Model } 4 \text { with covariance between Low } \\
\text { /High Arousal and Low Arousal/Self- } \\
\text { focus constrained equal }\end{array}$ & 402.90 & 130 & 17.93 & 16 & NS \\
\hline 11 & $\begin{array}{l}\text { Model } 4 \text { with covariance between Low } \\
\text { /High Arousal, Low Arousal/Self-focus, } \\
\text { and High Arousal/Self-focus } \\
\text { constrained }\end{array}$ & 402.99 & 131 & 17.99 & 17 & NS \\
\hline
\end{tabular}

Note. $\Delta \chi^{2}=$ difference in $\chi^{2}$ values; $\Delta \mathrm{df}=$ difference in degrees of freedom.

aAll models compared with Model 1 . 
and adult four-factor savoring models were combined and all parameters were allowed to be freely estimated (Model 1, Table 2.5). An omnibus test of the equality of factor loadings, variances, and covariances across the two age groups was tested and revealed a statistically significant difference (Model 2). Separate tests of invariance by first, factor loadings (Model 3); second, variances (Model 4); and third, covariances, revealed a significant difference only at the level of covariances (Model 5), as reflected by a significant difference in the chi square value between Model 5 and Model 1. The equality constraint on the covariance between dampening and low arousal yielded a significant difference (Model 6); the covariance was then allowed to be freely estimated. The covariance between dampening and high arousal also yielded a significant difference (Model 7); the covariance was then allowed to be freely estimated. Thirdly, the covariance between dampening and self-focus also yielded a significant difference (Model 8); this covariance was then allowed to be freely estimated. The remaining covariances (i.e., between low and high arousal, low arousal and self-focus, and high arousal and self-focus) were constrained to be equal, but no significant differences were obtained in these cases (Model 11).

As noted above, all factor loadings and factor variances were found to be invariant between adolescents and adults, while three covariances were not. As seen in Figure 2.2., the relationships between dampening and the other three factors (low and high arousal, and selffocus) were significantly different between groups. Adolescents showed a stronger relationship

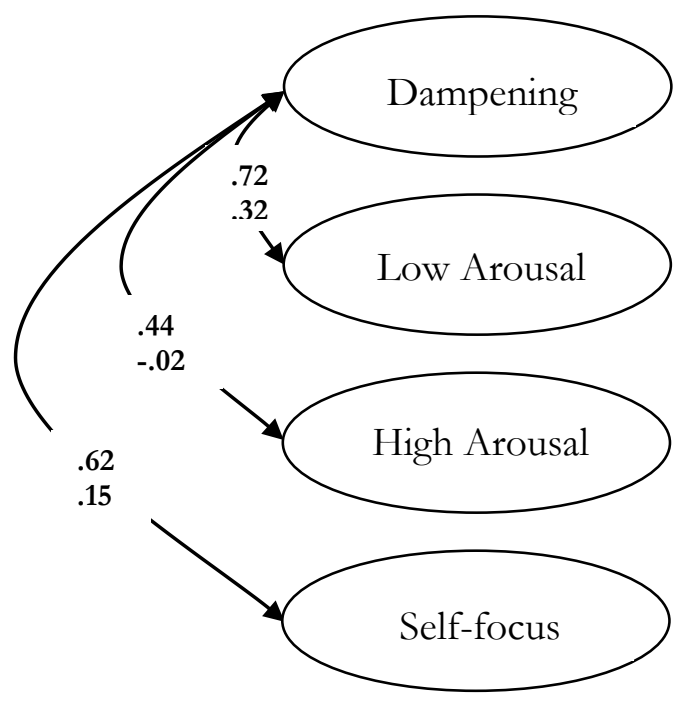

Figure 2.2. Correlations between dampening savoring and low arousal, high arousal, and selffocus savoring for adolescents (top correlation value) and adults (bottom correlation value). Note. All correlations were significantly different at $p<.001$. 
for all of these covariances than adults. The adolescent standardised covariances for dampening and low arousal (.72), dampening and high arousal (.44), and dampening and self-focus (.62) were significantly greater $(p<.001)$ than the standardised covariances for adults $(.32,-.02, .15$ respectively).

In sum, a sequence of invariance tests supported the second hypothesis that the adult sample would evidence a more differentiated model of savoring than the adolescent sample. It was found that adults differentiated their dampening savoring from high arousal, low arousal, and self-focus savoring more than adolescents.

\section{Gender and Age: Do gender and age influence savoring strategies?}

The third and final aim of this study was to test for gender and age mean group differences on savoring with both the adolescent and adult samples. The three hypotheses were that: (1) females would endorse positive savoring strategies to a greater extent than males, (2) males would endorse negative strategies to a greater extent than females, and (3) adolescents would use more negative savoring than adults, while adults would use more positive savoring than adolescents.

Before beginning analyses it was noted that the adult sample was $83 \%$ females and the adolescent sample was more balanced with $51 \%$ females. To more accurately test the effect of gender on savoring by adults, adult females were randomly selected to equal the number of males in the sample from four age categories. ${ }^{14}$ The number of adults from each age category was: 112 16 to 29 year-olds; 9830 to 44 year-olds; 8245 to 59 year-olds, and; 4060 to 85 year-olds. These individuals constituted a total adult sample of 332 with 166 males and 166 females. After selecting an equal number of adult males and females, the adolescent and adult samples were combined and a 2 (gender) by 2 (age: adolescent and adult) MANOVA was used to test the effects of gender and age on dampening, low and high arousal, and self-focus everyday savoring.

${ }^{15}$ Descriptive statistics (i.e. means and standard deviations) for dampening, low arousal, high arousal, and self-focus savoring by adolescent and adult males and females are presented in Table 2.6.

\footnotetext{
${ }^{14}$ As only $58 \%$ of the adult sample was from New Zealand while $100 \%$ of the adolescent sample was from New Zealand, I conducted another MANOVA that only included the New Zealand adults. All mean group differences for gender and age discussed in this section were confirmed with the subsample of New Zealand adults. 15 All MANOVA analyses used the Pillai's trace test statistic.
} 
Table 2.6. Means and Standard Deviations for Adolescents $(N=463)$ and Adults $(N=332)$ on Four Factors of Savoring.

\begin{tabular}{|c|c|c|c|c|c|c|c|c|c|c|c|c|}
\hline \multirow[t]{2}{*}{ Ages } & \multicolumn{2}{|c|}{ Males } & \multicolumn{2}{|c|}{ Females } & \multicolumn{2}{|c|}{ Total } & \multicolumn{2}{|c|}{ Males } & \multicolumn{2}{|c|}{ Females } & \multicolumn{2}{|c|}{ Total } \\
\hline & M & $\mathrm{SD}$ & M & SD & M & SD & M & SD & M & SD & M & SD \\
\hline \multicolumn{7}{|c|}{ Dampening Savoring } & \multicolumn{6}{|c|}{ Low Arousal Savoring } \\
\hline 13-15 & 3.78 & 1.34 & 3.94 & 1.41 & 3.88 & 1.38 & 3.67 & 1.34 & 3.80 & 1.33 & 3.74 & 1.33 \\
\hline $16-85$ & 3.07 & 1.38 & 2.75 & 1.25 & 2.91 & 1.32 & 3.78 & 1.38 & 3.88 & 1.29 & 3.83 & 1.34 \\
\hline \multicolumn{7}{|c|}{ High Arousal Savoring } & \multicolumn{6}{|c|}{ Self-focus Savoring } \\
\hline $13-15$ & 4.14 & 1.13 & 4.51 & 1.27 & 4.36 & 1.25 & 4.11 & 1.28 & 4.14 & 1.33 & 4.13 & 1.31 \\
\hline $16-85$ & 4.66 & 1.25 & 5.16 & 1.20 & 4.91 & 1.24 & 4.55 & 1.32 & 4.93 & 1.23 & 4.74 & 1.29 \\
\hline
\end{tabular}

Note. Adolescents included: 13 year-olds ( $N=121: 52$ males, 69 females); 14 year-olds $(N=242: 103$ males, 139 females); and 15 year-olds ( $N=97: 33$ males, 64 females). Adults included: 16 to 29 year-olds ( $N=112: 56$ male, 56 female); 30 to 44 year-olds ( $N=98: 49$ male, 49 female); 45 to 59 years-olds ( $N=$ 82: 41 male, 41 female); and 60 to 85 year-olds ( $N=40: 20$ male, 20 female).

\section{Hypothesis 3: Females would endorse amplifying savoring strategies (i.e. low} arousal, high arousal, and self-focus) to a greater extent than males. Multivariate results indicated a significant effect by gender, $F(4,788)=6.69, p<.001$, partial $\eta^{2}=.03$. Univariate effects revealed females $(M=4.83, S D=.06)$ reported employing high arousal strategies more than males $\left(M=4.40, S D=.07, F(1,791)=23.94, p<.001\right.$, partial $\left.\eta^{2}=.03\right)$. Females $(M=4.53$, $S D=.06)$ also reported using more self-focus strategies compared to males $(M=4.33, S D=.07$, $F(1,791)=7.07, p<.05$, partial $\left.\eta^{2}=.01\right)$. Focused $t$-tests indicated that females used more high arousal strategies, in both adolescence $(M=4.51, S D=1.27, t(458)=-3.21, p<.01$, representing a small effect size $\left.{ }^{16} r=.15\right)$ and adulthood $(M=5.16, S D=1.20, t(330)=-3.68, p<.001, r=$ $.20)$, than males $(M=4.14, S D=1.13 ; M=4.66, S D=1.25$ respectively). Gender in adolescence was not found to affect self-focus strategies, however, the endorsement of self-focus strategies was higher for adult females $(M=4.93, S D=1.23)$ compared to adult males $(M=4.55, S D=$ $1.31, t(330)=-2.26, p<.01, r=.15)$. No significant gender effect was found for dampening or low arousal savoring. Generally, gender results were congruent with the hypothesis, as they

\footnotetext{
${ }^{16}$ Effect size was calculated using Andy Field's (2009) suggested Pearson's correlation coefficient rather than Cohen's $d$ because $r$ is constrained to be between 0 (no effect) and 1 (a perfect effect). The equation used for calculating $r$ was: $r=$ the square-root of the squared $t$ statistic divided by the squared $t$ statistic plus degrees of freedom, originally presented by Rosenthal (1991, p. 19).
} 
indicated both adolescent and adult females support using high arousal strategies (i.e. one of the types of amplifying savoring) more than adolescent and adult males, however, only adult females used self-focus strategies more than adult males, and there were no differences in low arousal savoring across gender.

\section{Hypothesis 4: Males would endorse dampening strategies to a greater extent than}

females. The hypothesis was not supported by the multivariate test for gender, as no effect was obtained for the subsequent univariate test for dampening savoring; however, a multivariate gender $\mathrm{x}$ age interaction was significant, $F(4,788)=3.46, p<.01$, partial $\eta^{2}=.02$, and the subsequent tests of univariate effects revealed a gender $\mathrm{x}$ age interaction only for dampening savoring, $F(1,791)=6.04, p<.05$, partial $\eta^{2}=.01$. A trend appeared where dampening savoring decreased with age, but adult males endorsed dampening savoring more than adult female, as seen in Figure 2.3. Focused $t$-tests indicated that males $(M=3.07, S D=1.37)$ used more

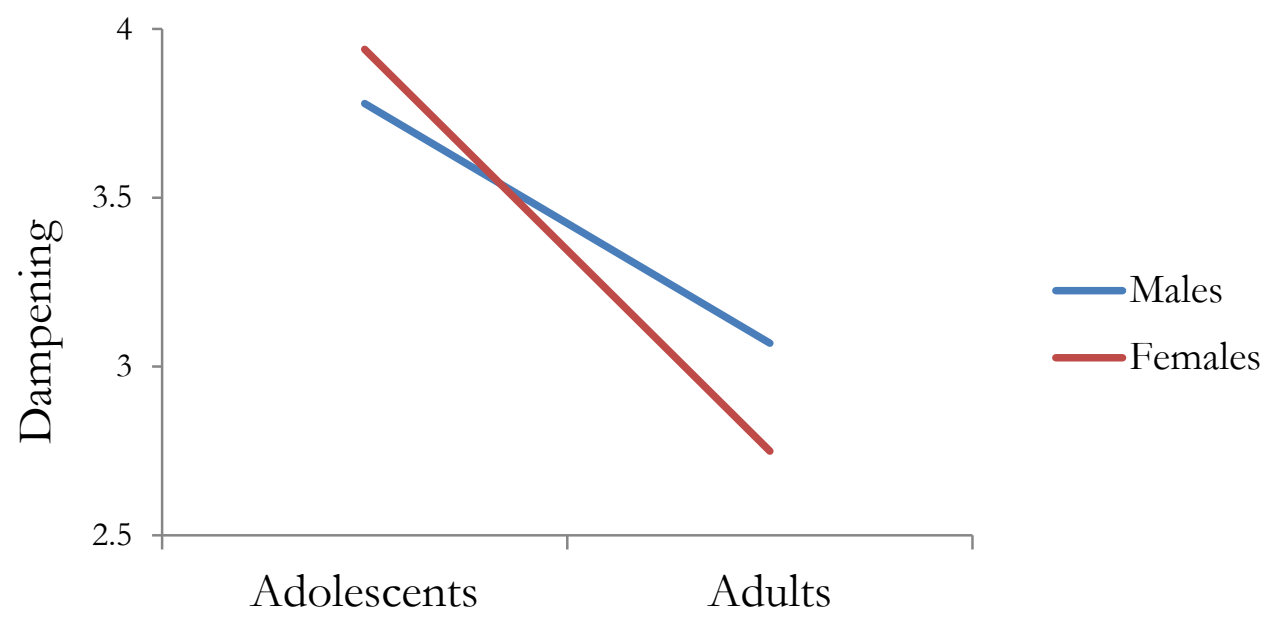

Figure 2.3. The interaction between gender and age on dampening savoring.

dampening savoring than females $(M=2.75, S D=1.25, t(330)=2.12, p<.05, r=.12)$, but only in adulthood, partially supporting the hypothesis that males would use negative strategies more than females.

Hypothesis 5: Adolescents would use more negative savoring, while adults would use more positive savoring. Multivariate results indicated a significant effect by age, $F(4,788)$ $=53.63, p<.001$, partial $\eta^{2}=.21$. Univariate effects revealed adolescents $(M=3.86, S D=.06)$ reported using more dampening strategies than the adults $(M=2.91, S D=.07), F(1,791)=$ 94.11, $p<.001, \eta^{2}=.12$, supporting the hypothesis. 
Also congruent with the hypothesis, it was found that adolescents $(M=4.32, S D=.06)$ employed less high arousal strategies than adults $(M=4.91, S D=.07), F(1,791)=44.49, p<$ $.001, \eta^{2}=.05$. Adolescents $(M=4.13, S D=.06)$ also used less Self-focus strategies than adults $(M=4.74, S D=.07), F(1,791)=42.57, p<.001, \eta^{2}=.05$, but there was no statistically significant effect by age for Low Arousal strategies. See Figure 2.4 for a comparison of Dampening, High Arousal, and Self-focus savoring between adolescents and adults.

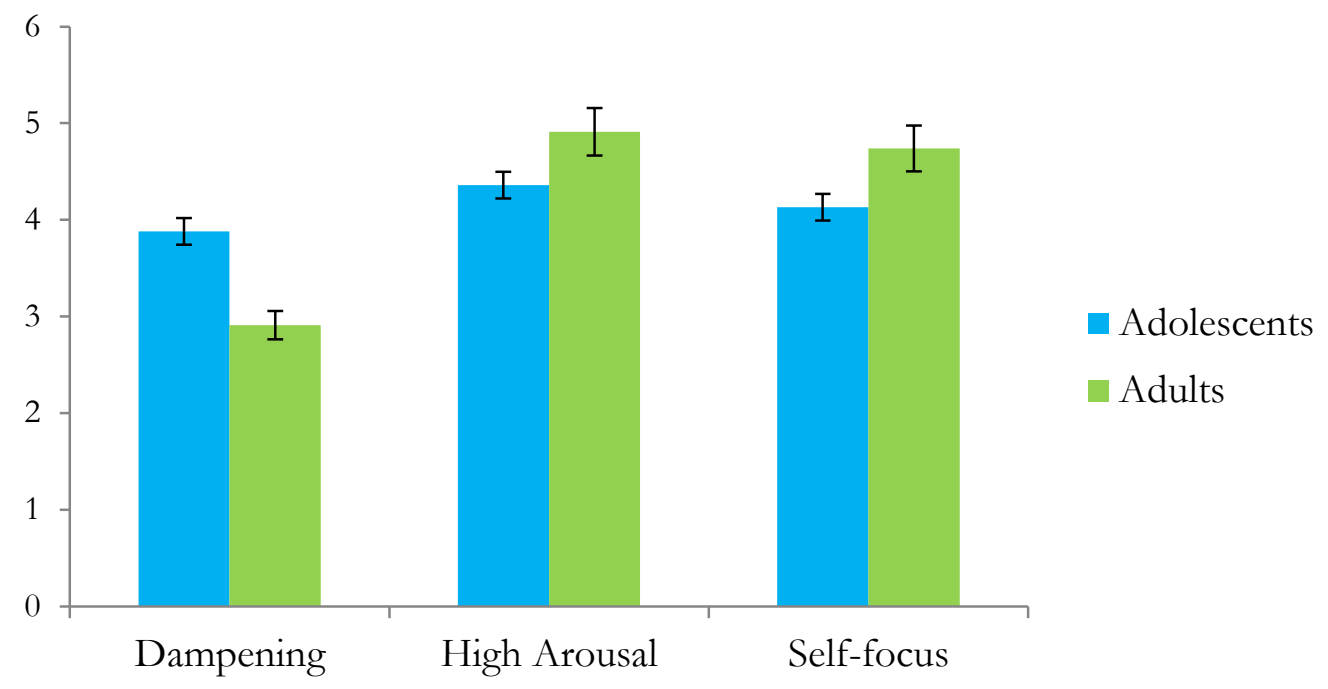

Figure 2.4. Dampening, high arousal, and self-focus everyday savoring strategies reported by adolescents and adults.

\section{Discussion}

The present study was an in-depth examination of everyday savoring of everyday positive life events by adolescents and adults. Three main research questions guided this examination: (1) Is the model of savoring similar for adolescents and adults? (2) Are there differences in the multi-dimensional nature of savoring strategies between adolescents and adults? and (3) Do gender and age influence savoring strategies? Each question will be discussed in order.

\section{Hypothesis 1: The configuration of everyday savoring of everyday positive events}

Findings were congruent with the hypothesis that the process of savoring everyday positive events would have a similar configuration for both adolescents and adults. One multidimensional model, with four factors and 13 items, best represented everyday savoring for both samples. This model described everyday savoring in terms of responding to positive events by cognitively dampening them, using behaviours to focus and absorb the moment, sharing or 
behaving excitedly, and by reflecting back on the self. These four factors were labelled dampening, low arousal, high arousal and self-focus savoring.

As was outlined in the pre-operational explication process, everyday savoring in this study was expected to yield fewer factors than the original Ways of Savoring, primarily because this study used a third of the original 60 items. The reduction in items restricted more complex modelling and a 10-factor structure similar to previous findings by Bryant and Veroff (2007) was unsupported. A limitation of the abridged everyday savoring scale is that the multidimensional nature of savoring is restricted. Although this is an understood, the everyday model supported by this study was able to include at least one item from each of the original 10 WOSC factors. In addition, practical implications of an abridged savoring scale include expedited data collection and less fatigued participants.

Questions arise from this chapter and the factor analysis in particular: Is the four factor model of savoring valid? Is dampening savoring really a negative form of savoring? Is low arousal, high arousal, and self-focus savoring really positive savoring-for both adolescents and adults? And are each of these factors differentially related to other variables? It is important to seek answers to these questions in order to accurately understand how savoring, in all its multidimensionality, functions.

Knowing how each factor functions, for adolescents and adults, will illuminate the importance of specific strategies for specific outcomes, highlight possible developmental differences, and - a psychometric consideration — it will assist parcelling in latent variable path models. Analyses were conducted at the item-level because the aim of this section was to understand the configuration of savoring. If the objective was to consider how savoring relates to other constructs, parcelling items could be advantageous, especially if working with a small sample size; for instance, if one is unable to meet an acceptable parameter to participant ratio (Little, et al., 2002). First, however, it is important to know how these item-level factors relate to other constructs if they are to be combined into meaningful parcels. This is the aim of Chapter 3 , to better understand how the savoring of everyday positive events functions for both adolescents and adults.

\section{Hypothesis 2: The structure of savoring everyday positive events}

Adolescents reported savoring more globally than adults. In other words adults differentiated their savoring strategies more than adolescents. In particular, adolescents' dampening savoring was more highly related to their low arousal, high arousal, and self-focus 
savoring than for adults, supporting the hypothesis that the adult sample would evidence a more differentiated model of savoring than the adolescent sample.

Recent research has found that happiness increases with age (Stone, Schwartz, Broderick, \& Deaton, 2010). The ability of adults to better differentiate their dampening savoring strategies from their positive amplifying strategies is a possible explanation for how happiness increases with age. Since adolescents do not differentiate dampening strategies from amplifying strategies as acutely as adults, they may inadvertently reduce their positive experience of everyday positive events. Adolescents may seek to savor an event by comparing it to another, for example, and find that instead of becoming happier it leaves them unhappy. Adults' knowledge for the functional difference between dampening and amplifying strategies - the awareness of dampening's detrimental effect on feeling good-may have been learned and developed over time. The next chapter delves into this issue by uncovering how the savoring factors relate to positive outcomes, which will indicate whether dampening does in fact relate to a reduction in happiness.

\section{Hypotheses 3 through 5: How gender and age influence the savoring of everyday positive events}

Congruent with the hypothesis that females would endorse more amplifying savoring strategies than males it was found that both adolescent and adult females endorsed more high arousal savoring than adolescent and adult males. Only adult females used more self-focus savoring than adult males, no difference was found for adolescents. Further, no difference in low arousal savoring was identified for either sample. Thus the gender difference for amplifying savoring of everyday positive events seems to be specific, rather than universal; it applies to particular strategies and sometimes to particular age groups.

The hypothesis that males would endorse negative strategies to a greater extent than females was partially supported, the relationship depended on age. Males reported using more dampening savoring than females only in adulthood, there were no differences in adolescence. The relatively equal endorsement of dampening savoring in adolescence may be related to the heightened rumination by females in adolescence. Nolen-Hoeksema (1994) has proposed that females' increased use of rumination compared to males begins in adolescence. On the other hand, it may simply be that developmental influences outweigh gender influences for the endorsement of dampening savoring in adolescence.

Age had a variable impact on savoring. Congruent with the fifth hypothesis, adolescents reported more dampening strategies than adults, and less amplifying savoring than adults. Adults 
used high arousal and self-focused savoring more than adolescents, while adolescents used more dampening savoring than adults. The finding that adolescents engage in more dampening savoring may help explain their vulnerability to psychopathology (Price \& Lento, 2001). The killjoy within dampening savoring has been associated with poorer outcomes in Western cultures, for example, it is used more by people with low self-esteem, and low self-esteem in adolescence predicts poorer mental and physical health in adulthood (Bryant \& Veroff, 2007; Trzesniewski, et al., 2006; Wood, et al., 2003). Further, the decreased use of amplifying savoring strategies compared with adults leaves adolescents lacking beneficial and influential positive emotions that are able to buffer against psychopathology (Garland, et al., 2010). Nevertheless, developmental and goal-related reasons for dampening positive emotions exist.

One possibility is that dismissing the convention of happiness is important to adolescent socio-emotional development. By dampening positive emotions adolescents work to form emotional autonomy from their parents, adopt a sense of maturity, and construct their personal and social identities (Azmitia, Syed, \& Radmacher, 2008). One study found that lower mood supports the development of capabilities in adolescence to disengage from goals. Building goaldisengagement capabilities led to a reduction in subsequent depressive symptoms over a year (Wrosch \& Miller, 2009). Another interesting finding is that wanting to decrease positive emotions by using dampening savoring may work better for particular goals; where future benefits outweigh immediate benefits individuals often choose to feel useful emotions even if they are unpleasant (Tamir, 2009; Tamir, Mitchell, \& Gross, 2008). This lean toward the negative during adolescents, although potentially useful, poses an increased risk for trouble behaviour that is considered developmentally normal, part and parcel with travelling through the teen years (Moffit, 1993; Moffit, Caspi, Harrington, \& Milne, 2002).

With the evidence that adolescents were not only higher dampening savorers, but were also lower amplifying savorers, questions arise as to how savoring everyday positive events relates to aspects of happiness and wellbeing. Understanding how amplifying savoring relates to happiness would provide insights into adolescent savoring; for instance, although high arousal savoring is used less in adolescence, it may be more influential for certain types of happiness compared to adults. Similarly, self-focus and dampening strategies may relate differently to happiness for adolescents and adults. In addition, low arousal savoring, which has not displayed differences by gender or age, may still be qualitatively different for adolescents and adults. Chapter 3 will examine these important questions. 
As noted earlier, a limitation of this study was that the adolescents and adults constituted separate samples. The adolescents did not become the adults in this study, and it is quite possible that adolescents from the Bay of Plenty in New Zealand would not grow up and be identical to the adults recruited for the international IWS on-line study. This sample difference mitigates the confidence in the developmental inferences identified here regarding savoring, which has been the majority of this discussion. Rather than the experience of savoring changing with age, adults studied today may have always been high amplifying savorers and low dampening savorers, even when they were adolescents. Further, it may be that the relationships and differences in this study reflect cohort effects, selection effects, or methodological differences; for example, although the 20-items analysed here were identical between samples, adolescents originally replied to a 30-item savoring scale and adults replied to a 20 -item scale, and adults were given fewer positive life events questions before their savoring questions. Ideally future extended longitudinal studies will include a measure of savoring to better inform development of these cognitive-behavioural abilities. Provided these disparities between samples, both groups still shared a similar structure of savoring, evidence that the construct of savoring is consistent and robust. The immediate next step for this thesis, however, will be met in Chapter 3 by investigating the relationships between everyday savoring, and a group of important outcome variables, in particular, satisfaction, happiness (i.e. hedonic wellbeing), and eudaimonic wellbeing for adolescents and adults. 


\section{CHAPTER 3}

\section{Concurrent Savoring and Wellbeing}

We suggest that true wisdom lies in learning to savor in ways that achieve both hedonia and eudemonia, without trading one form of happiness for the other.

(Bryant \& Veroff, 2007, p. 214)

When savoring is mentioned in the literature, it is often defined by its relationship with positive emotion: savoring is the self-regulation of positive emotions, typically manifested by generating, maintaining and enhancing positive affect by attending to positive events from the past, present, or future (Bryant,1989, 2003; Bryant \& Veroff, 2007). At a more general level, however, savoring is proposed to both moderate and mediate positive experience (Bryant, Chadwick, \& Kluwe, 2011; Bryant, et al., 2008; Bryant \& Veroff, 2007; Jose, et al., 2011). There is little research, however, that has investigated the impact of savoring on important wellbeing indicators like life satisfaction, overall happiness, having meaning in life, or psychological and social wellbeing. To the best of my knowledge, no other study has researched the related impact of everyday savoring strategies on both hedonic and eudaimonic wellbeing, or the moderational properties of savoring on the relationship between hedonic and eudaimonic wellbeing with adolescents or adults. This chapter reports my efforts to investigate these relationships and the possible impact of age, while also testing the validity of the four styles of everyday savoring identified in Chapter 2.

Bryant and Veroff (2007) originally categorised savoring strategies by their underlying response mechanism, either cognitive (e.g. self-congratulations) or behavioural (e.g. behavioural expression), or a combination of both (e.g. sensory-perceptual sharpening). Analyses in Chapter 2 confirmed categorisation of the savoring of everyday positive events into four styles: dampening, low arousal, high arousal, and self-focus savoring. Dampening and self-focus savoring factors were primarily composed of cognitive mechanisms, while low and high arousal savoring factors were primarily composed of behavioural mechanisms. After finding that both adolescents and adults use four basic styles of savoring for their everyday positive events in Chapter 2, the next question is whether this organisation of everyday savoring is meaningful by observing its relationship with wellbeing.

The overarching purpose of this investigation is twofold: (1) to observe the relationships between savoring everyday positive events and multiple wellbeing indicators to reveal the utility of specific strategies; and (2) to observe the similarities and differences in these relationships for both adolescents and adults to uncover the potential impact of development on the use of 
savoring everyday positive events. By investigating these questions I am answering the overarching question of the chapter: is the four-style categorisation of everyday savoring meaningful and psychometrically robust.

\section{Brief review: Indicators of wellbeing}

Several indicators of wellbeing can be grouped by hedonic or eudaimonic perspectives. Hedonic wellbeing is often conceptualised as subjective wellbeing, which is a combination of emotional and cognitive wellbeing, in other words, a combination of positive affect, or the ratio of positive affect to negative affect, and life satisfaction (Diener, 1984; Diener, Scollon, et al., 2009; Kahneman, et al., 1999). In the present study, adolescents' cognitive wellbeing was measured with an earlier version of the Satisfaction with Life scale (Diener, et al., 1985), while adults' cognitive wellbeing was measured with a more contemporary and longer version, called the Temporal Satisfaction with Life scale (Pavot, Diener, \& Suh, 1998).

The theoretical rationale of hedonic wellbeing is that individuals act to maximise pleasure and reduce pain. While the composition of eudaimonic wellbeing is less agreed upon, some of the primary themes involved in the definition are: meaning, engagement, autonomy, personal growth, environmental mastery, and social connection (Frankl, 1963; Jahoda, 1958; Keyes, 2003; Peterson, et al., 2005; Ryan \& Deci, 2001; Ryff, 1989; Ryff \& Singer, 1998; Waterman, 1993). The theoretical foundation of eudaimonia is that people are thought to strive to live by personally adopted values. In other words, hedonic wellbeing is defined by the attainment of pleasure and avoidance of pain, while eudaimonic wellbeing is defined by how much a person is fully functioning in relation to his or her life goals or values (Ryan \& Deci, 2001).

Emotional and cognitive wellbeing (i.e. hedonic wellbeing) have been well researched by asking individuals about general happiness, specific emotions, and life satisfaction (Diener, et al., 1985; Lyubomirsky, King, et al., 2005; Pavot, et al., 1998; Watson, Lee, \& Tellegen, 1988). The components of emotional and cognitive wellbeing are considered independent contributors to subjective wellbeing shown by their disparate levels across the life-span; life satisfaction tends to increase with age and positive affect tends to decrease (Diener, et al., 2006; Diener, Lucas, et al., 2009; Diener, et al., 1999), although a more recent study has found that positive affect increases from the age of 50 (Stone, et al., 2010). Although life satisfaction has been extensively researched with adults, the literature on adolescent life satisfaction is scanty. However, similar to adults, life satisfaction is not only an important positive predictor, for example, of good physical health, it also helps protect against internalising and externalising behaviours in adolescence (see Proctor, Linley, \& Maltby, 2009). Both samples in the current study reported their general happiness 
(closely related to frequent positive affect) on the Subjective Happiness scale (Lyubomirsky \& Lepper, 1999).

The utility of frequent positive emotions (or general happiness) has been well researched. They appear to predict success in several life-domains including family and work, contribute to longevity, and provide immunity to the common cold (Carstensen, et al., 2010; Cohen, et al., 2003; Danner, et al., 2001; Lyubomirsky, King, et al., 2005; Xu \& Roberts, 2010). Some research, however, finds emotional wellbeing is not enough. A central figure who has argued for recognising the distinction between hedonic and eudaimonic wellbeing, Keyes (2005a) has designed a measure of emotional, psychological, and social wellbeing, which he terms the Mental Health Continuum - which can classify people from languishing, through moderate functioning, to flourishing mental health. The psychological and social measures are used to gauge level of functioning or eudaimonia, and were used to measure adolescent eudaimonia in the present study.

Keyes has found that individuals with high levels of hedonic wellbeing but only moderate eudaimonic wellbeing are twice as likely to suffer from a mental illness as people with high levels of both hedonic and eudaimonic wellbeing (Keyes \& Annas, 2009). Keyes further argues that the majority of people, young and old, report feeling good, but a majority also report functioning poorly; meaning that people endorse high levels of emotional wellbeing, while simultaneously endorsing low levels of psychological and social wellbeing (Keyes, 2006, 2007). Anything less than flourishing, which is defined as a combination of high hedonic and eudaimonic wellbeing, is associated with, for example, lost work productivity, increased disability, and increased healthcare use from those with and without a corresponding mental illness. These findings emphasise the importance of measuring eudaimonic indicators of wellbeing.

Recently researchers suggested another type of happiness that is theoretically distinct from both hedonia and eudaimonia. Peterson and colleagues (2005) have specified three orientations to happiness: pleasure, meaning, and engagement or flow (Csikszentmihalyi, 1991). Pleasure is hedonic, and meaning is eudaimonic, whereas engagement seems to exist outside these two domains. Although Waterman (1993) has defined engagement as a combination of hedonic and eudaimonic, Peterson and colleagues argue that flow is non-emotional and noncognitive, making it distinct from both hedonia and eudaimonia (Peterson, Ruch, Beermann, Park, \& Seligman, 2007). For the purpose of this study, meaning alone, rather than in combination with engagement, was used to represent eudaimonic wellbeing for the adult sample. Meaning predicts life satisfaction beyond pleasure alone (Peterson, et al., 2005; Vella-Brodrick, 
Park, \& Peterson, 2009), and having meaning in life is linked to good mental health, lower incidences of psychopathology, and increases in longevity, supporting Frankl's (1963) earlier work on the central role that meaning has in cultivating positive mental and physical health (Adams, Bezner, Drabbs, Zambarano, \& Steinhardt, 2000; Boyle, Barnes, Buchman, \& Bennett, 2009; Debats, Van der Lubbe, \& Wezeman, 1993; Zika \& Chamberlain, 1992).

The positive psychology field is enjoying an ongoing discussion about hedonic and eudaimonic approaches to wellbeing (Delle Fave \& Bassi, 2009; Kashdan, et al., 2008; Keyes \& Annas, 2009; Waterman, et al., 2008)—a discussion that has been around since long before the inception of positive psychology (McMahon, 2006). Often the argument attempts to specify whether hedonic and eudaimonic wellbeing are separate dimensions, or aspects of the same continuum. Several studies structure hedonic wellbeing as leading to eudaimonic wellbeing, which was how hedonic and eudaimonic wellbeing were conceptualised for the last research question of this chapter (Fredrickson, et al., 2008; King, Hicks, Krull, \& Del Gaiso, 2006; Lyubomirsky, King, et al., 2005; Waugh \& Fredrickson, 2006). There is no single accepted, or unified, theory concerning these issues, but there is agreement and evidence that both hedonia and eudaimonia are important indicators of a life being well lived (Della Fave, Brdar, Freire, Vella-Brodrick, \& Wissing, 2011; Keyes, 2007; Vella-Brodrick, et al., 2009).

\section{Savoring's association with wellbeing}

New evidence supports the unique associations between specific savoring strategies and individual components of hedonic wellbeing (Quoidbach, et al., 2010). A group of 282 adults completed online measures that included 6 brief positive emotional scenarios, some rarer than others (e.g. winning $\$ 1,500$ on lottery, and a friend winning a trip for two to a paradise island), representing six emotional experiences (excitement, gratitude, awe, contentment, joy, and pride). The adults were then provided with eight broad response strategies, each represented by a single item, to each of the six scenarios. Of the eight response strategies, four were positive-labelled savoring: behavioural display, capitalization (i.e. sharing with others), being in the present, and positive mental time travel (i.e. reminiscing); and four were negative-labelled dampening: suppression, distraction, fault finding, and negative mental time travel.

Quoidbach et al. (2010) found that different savoring and dampening strategies independently related to emotional and cognitive wellbeing. Positive mental time travel and being in the present were positively associated with positive affect, whereas distraction was negatively associated with positive affect. Sharing with others was positively associated with life satisfaction, but finding fault and negative mental time travel were negatively associated with life 
satisfaction. Given these findings and findings that adults who used a broader range of savoring strategies were happier, Quoidbach et al. concluded that positive regulatory diversity (i.e., using multiple savoring strategies, rather than only a few) leads to greater happiness. However, Quoidbach et al. did not research eudaimonic wellbeing, processes that parallel self-focused savoring, everyday positive events, or adolescents' savoring strategies. Nonetheless, applying these results to the adults of this chapter suggests three possible predictions: (1) low arousal savoring (i.e. absorbing the moment and blocking outside distractions with sensory perceptual sharpening) will relate positively to emotional wellbeing, (2) high arousal savoring (i.e. sharing with others and behavioural expression) will relate positively to life satisfaction, and (3) dampening savoring will relate negatively to both.

\section{Does age moderate the relationship between savoring and wellbeing?}

The capacity to savor appears to be developmental — it becomes less global and more differentiated as people age. Children manifest a global ability to savor without respect to time, adolescents seem to be able to savor specifically in the past, present and future, and adults make clear distinctions between these savoring time perspectives, e.g., adults have a preference for one of the savoring time perspectives (for a review, Bryant \& Veroff, 2007). Following this line of research and the findings from Chapter 2 that dampening savoring was more strongly related to low arousal, high arousal, and self-focused savoring for adolescents than adults, I predicted: adolescents' savoring types will share similar relationships to wellbeing indicators, while adults will, in comparison, manifest more differentiated relationships between types of savoring and specific wellbeing outcomes. I expected these differences to be slight, rather than pervasive, in line with Bryant and Veroff's discussion of how adolescents are able to distinguish savoring in different time perspectives, but it was expected that adults would make clearer types distinctions. To explain this hypothesis further, I turn to the literature on aging, emotion, and happiness.

New evidence supports the view that the capacity to savor the moment increases during later life. Recent results from over 340,000 telephone interviews with individuals in the US reveal that wellbeing, especially positive emotions, steadily increases from the age of 50 (Stone, Schwartz, Broderick, \& Deaton, 2010). Over the adult life-span, from 18 to 85 years of age, individuals' appraisal of their happiness resembles a " $U$ " shaped curve; it begins high, dips from the late 30 s until age 50 , when it begins to climb, and by age 80 individuals have exceeded all younger reports of happiness. These findings are supported by Carstensen and colleagues' (2010) cross-sectional and longitudinal experience sampling of 178 adults from 18 to 94 years old. Findings from over a decade of their research show that aging is associated with greater 
emotional wellbeing, specifically, an increase in the ratio of positive to negative emotions, and greater stability in emotional experiences.

Another study using experience sampling investigated the changes in motivations to feel positive and negative emotions from 378 individuals, 14 to 86 years old (Riediger, et al., 2009). Riediger and colleagues asked about emotional experience at the same time as asking about whether they wanted to maintain, enhance, or dampen positive and negative emotions. Adolescents wanted to maintain or enhance negative affect and dampen positive affect more than adults (called contra-hedonic motivations). Conversely, adults held pro-hedonic motivations, they wanted to either maintain positive affect or dampen negative affect more than adolescents. This motivational change parallels the reports of increasing day-to-day emotional wellbeing with age. It also follows findings reported from Chapter 2 that showed adults use more amplifying savoring strategies and fewer dampening savoring strategies than adolescents. The present chapter will extend this finding by investigating the ranking of each savoring strategy within each age group. From the findings in Chapter 2 and those presented by Riediger and colleagues (2009), it was predicted that adolescents would rank their dampening and low arousal savoring similarly, and that generally the ranked differences for adults would be greater than for adolescents, i.e., adults would make a greater distinction between each savoring strategy than adolescents in line with increased differentiation between savoring strategies across development.

One plausible explanation for the increase in happiness with older age relates to the shifting meaning of happiness over time. As adults age they tend to transfer their definition of happiness from excitement to peacefulness (Kamvar, Mogilner, \& Aaker, 2009; Mogilner, Kamvar, \& Aaker, 2011). Generally, adults under the age of 20 are five times more likely to express their happiness as excited, rather than peaceful, whereas adults over the age of 50 are two times more likely to express their happiness as peaceful, rather than excited. Mogilner and colleagues (2011) argue that a shift in time perspective causes the shift in happiness and they worked to support this theory by asking young and older adults to participate in present-focused meditation. They found that young adults' definition of happiness as peacefulness increased for the meditation group compared to the control group. For older adults, however, there was no difference in their definition of happiness as peacefulness between the meditation and control groups, indicating that individuals of older age tend to embrace a chronic present focus.

Previous studies support these findings by showing a strong and consistent link between older age and an enhanced awareness of the present. In other words, older individuals' keen awareness of the present moment makes old age more emotionally meaningful (Carstensen, 
Fung, \& Charles, 2003). These conclusions are consistent with experimental evidence from university students anticipating graduation, which found that becoming more aware of the approaching end of an experience boosts the appreciation of the present (Kurtz, 2008). Taken together, increasing age influences emotional wellbeing, motives for feeling emotions, actual happiness definitions, and time perspective-indicating that as people age, they become increasingly capable of savoring, and in particular, savoring the moment. It was predicted in this study that although Chapter 2 found no mean differences in the reported levels of low arousal savoring between adolescents and adults, the apparent increasing capability to savor the moment would have a positive impact on wellbeing for adults, more so than for adolescents.

\section{Hypotheses: Validity of adolescent and adult everyday savoring}

The importance of emotional, cognitive, and functional wellbeing to positive health has been established. Yet, the relationships between savoring and wellbeing, for instance, how savoring impacts wellbeing-positively or negatively_and which types of savoring processes impact which types of wellbeing are still unknown. No study has yet analysed the unique contribution of savoring strategies to both hedonic and eudaimonic wellbeing. Nor has any study observed all of these variables within a single analysis to allow for a more realistic representation of the dynamic impact of savoring on positive health by both adolescents and adults. It might be that one style of savoring everyday positive events is more effective at predicting cognitive wellbeing, while another is better at predicting eudaimonia - indicating that a varied use, rather than a singular use, of savoring strategies might better contribute to a sense of overall wellbeing. In addition, adolescents may use different strategies for amplifying or dampening eudaimonic wellbeing than adults, for example, implying that developmental differences in the utility of specific savoring strategies are important to recognize.

However, before further outlining my expectations for this study, it is worth drawing attention to an issue I first raised in Chapter 2. Although all measures between the adolescent and adult samples were similar, they were not all identical. In the current study, adolescents responded to the original satisfaction with life scale (Diener, et al., 1985), whereas adults responded to a newer extended version (Pavot, et al., 1998); and adolescents responded to a eudaimonic measure on the Mental Health Short Form (Keyes, 2005b, 2006), whereas adults responded to the eudaimonic component, or orientation toward meaning, on the Orientation to Happiness scale (Peterson, et al., 2005). The happiness scale, which measures global happiness, was identical, and the savoring scales were those reported in Chapter 2. I acknowledge that direct comparisons between adolescents and adults were not possible and therefore I did not perform 
any analyses that would ignore this knowledge (e.g. performing equality constraints between adolescent and adult path models). I do, however, present adolescent and adult findings side-byside to facilitate observing their similar and different ways of using savoring to effect their life satisfaction, happiness, and eudaimonia.

To begin understanding the relationship between savoring strategies and wellbeing, I first examined the relative ranking of the four savoring strategies for adolescents and adults. Chapter 2 revealed that adults use more positive savoring and less negative savoring than adolescents. As a descriptive tool, I now compared the preference of each savoring strategy, within each age group; for example, dampening savoring was sequentially compared to each of the other three strategies to examine whether respondents agreed to using dampening more or less than low arousal, high arousal, and self-focus to savor their everyday positive events. I expected that adolescents would agree to having used the amplifying savoring strategies of high arousal and self-focus above dampening savoring, but that there would be no difference between dampening and low arousal savoring. I also expected adults would evidence significant differences in their endorsement of each strategy in line with savoring becoming increasingly differentiated with age, and that all the positive strategies would rank above dampening savoring, as invariance testing in Chapter 2 revealed weaker relationships between dampening and each of the positive savoring styles for adults. Overall, this investigation questions whether individuals' endorsement of particular savoring strategies would parallel the savoring associations revealed in Chapter 2.

Next, I examined the relationships between specific savoring strategies and specific types of wellbeing, and their similarities and differences by age. Would dampening, low arousal, high arousal, and self-focus strategies uniquely relate to various indicators of adolescent and adult wellbeing? In examining the validity of the abridged WOSC, I generally expected dampening savoring would relate negatively to satisfaction, happiness, and eudaimonia, while the more amplifying savoring strategies would relate positively to the three wellbeing indicators for both groups. Although dampening savoring, which includes kill-joy thinking, is assumed to be associated with a reduction in happiness, this assumption has not been thoroughly tested.

Specifically, I hypothesised that adolescents' and adults' low arousal savoring would relate differently to wellbeing. The apparent increasing capability to savor the moment is congruent with adults' positive use of low arousal savoring that includes absorbing the moment and blocking out distractions. Given the findings from Chapter 2 that adolescents do not discriminate their low arousal strategies from their dampening strategies to the same degree as adults and that adolescents want to dampen positive emotions more than adults, I expected that 
adolescents would evidence a negative relationship between low arousal and wellbeing. On the other hand, given adults' shifting meaning of happiness to peacefulness, increasing focus on the present, and desire to maintain positive emotions, I expected adults would evidence a positive relationship between low arousal savoring and wellbeing.

After I examined the direct relationships from savoring to wellbeing, I explored each savoring strategy as moderating the impact of hedonic wellbeing on eudaimonic wellbeing. Congruent with the broaden-and-build theory of emotions where, for example, pride leads to mastery, contentment and interest open up meaning, and love leads to social connection, the present study modelled hedonia as predicting eudaimonia (Fredrickson, 1998, 2001; Waugh \& Fredrickson, 2006). It has similarly been argued that functioning well leads to positive emotions and that these two aspects of wellbeing both affect one another; however, for simplicity and the purposes of this study only hendonia predicted eudaimonia (Fredrickson, 2002). As reviewed previously, savoring is proposed to moderate positive experience (Bryant, et al., 2011; Bryant \& Veroff, 2007), but I know of only one study that empirical investigated this proposal (Jose, et al., 2011). To my knowledge, this is the first study to examine the moderating role of savoring on hedonic and eudaimonic wellbeing. It is expected that amplifying savoring will enhance the relationship between hedonia and eudaimonia, while dampening will diminish the positive impact of hedonia on eudaimonia for both age groups.

The present chapter had four main aims: (1) to examine the relative ranking of savoring strategies for both adolescents and adults; (2) to investigate the discriminant validity of savoring strategies by their relationship to indicators of wellbeing, (3) to explore the similarities and differences in these relationships between adolescents and adults, and (4) to explore potential savoring moderators for the relationship between hedonic and eudaimonic wellbeing. From these aims, I formed four research questions and related hypotheses:

\section{(1) Do adolescents and adults similarly rank their endorsement of savoring strategies?}

Hypothesis 1: Adolescents would endorse high arousal and self-focus savoring more than dampening and low arousal savoring, however, the ranked differences between dampening and low arousal savoring and high arousal and self-focused savoring would be weaker than for adults, and there would be no rank difference in dampening and low arousal savoring for adolescents.

Hypothesis 2: Adults would endorse each savoring strategy differently, with dampening savoring being the lowest endorsed strategy.

\section{(2) Do savoring strategies uniquely relate to wellbeing?}


Hypothesis 3: Dampening savoring would predict a decrease in wellbeing. Specifically, since dampening savoring and evaluating life satisfaction are cognitive processes, it is expected that dampening savoring would be associated with a decrease in life satisfaction as previously evidenced (Quoidbach, et al., 2010).

Hypothesis 4: High arousal and self-focused savoring would predict an increase in wellbeing. Specifically, since self-focused savoring includes cognitive savoring strategies, it was expected that self-focused savoring would be associated with an increase in life satisfaction.

(3) Does age appear to influence the savoring and wellbeing relationships?

Hypothesis 5: Adolescents' dampening and low arousal savoring would relate similarly_-both would manifest a negative relationship—with the wellbeing indicators. Hypothesis 6: Adults' low arousal savoring would be positively associated with wellbeing.

(4) Do savoring strategies moderate the relationship from hedonic to eudaimonic wellbeing?

Hypothesis 7: Adolescents' dampening and low arousal savoring would diminish the positive impact of hedonic wellbeing on eudaimonic wellbeing.

Hypothesis 8: Adults' dampening savoring would diminish the impact of hedonic wellbeing on eudaimonic wellbeing.

Hypothesis 9: Adolescents' high arousal and self-focused savoring would enhance the relationship between hedonic and eudaimonic wellbeing.

Hypothesis 10: Adults' low arousal, high arousal, and self-focused savoring would enhance the relationship between hedonic and eudaimonic wellbeing.

\section{Method}

\section{Participants and Procedure}

The participants and procedure were identical to Chapter 2. During the same sessions described in Chapter 2, where adolescents and adults reported on savoring, adolescents also reported on life satisfaction, global happiness, and social and psychological wellbeing. Adults reported on life satisfaction, global happiness, and meaning. As with Chapter 2, this study's participants were 463 school students, 13 to 15 years old $(M=13.95,51 \%$ of the sample was composed of females), and 980 adults from 16 to 84 years of age $(M=39,83 \%$ of the sample was comprised of females) participating through an internet survey. Adolescents were 64\% European New Zealand/Pakeha, 34\% were Māori , .7\% were Pasifika, .9\% were Asian, 1\% 
indicated "Other", and 14 adolescents omitted ethnicity, and $83 \%$ of adults were of White or European decent.

\section{Measures}

Savoring. The savoring measure was identical to that described in the previous chapter for both adolescents and adults. The confirmatory factor analysis from Chapter 2 found that a four factor solution of savoring everyday positive events fit the data well: dampening (e.g., 'I told myself why I didn't deserve this good thing'), low arousal (e.g., 'I thought only about the present-got absorbed in the moment'), high arousal (e.g., 'I looked for other people to share it with'), and self-focused savoring (e.g., 'I reminded myself how lucky I was to have this good thing happen to me'). The model fit indices for adolescents $\left(\chi^{2} / \mathrm{df}=2.55, \mathrm{CFI}=.93\right.$, RMSEA $=$ $.06, \mathrm{sRMR}=.04)$ and adults $\left(\chi^{2} / \mathrm{df}=4.16, \mathrm{CFI}=.94, \mathrm{RMSEA}=.06, \mathrm{sRMR}=.04\right)$ were acceptable.

Life satisfaction. The adolescents and adults responded to questions about their satisfaction with life on very similar measures, theoretically and structurally; however, the version the adults answered was an extension of the version the adolescents answered. On the extended version, there were ten more items. The extra items allow one to conceptualise adult satisfaction with life as multidimensional with respect to temporal orientation. However, the average was taken from the total satisfaction with life scale, for both samples, and analysed to investigate unidimensional satisfaction with life (i.e. ranging from unsatisfied through to satisfied with life).

Adolescents responded on a 7-point Likert scale to the 5-item Satisfaction with Life Scale (Diener, et al., 1985; W. Pavot \& Diener, 2008) that measures global life satisfaction; for example, 'In most ways my life is close to my ideal' ( $1=$ strongly disagree; $4=$ neutral; $7=$ strongly agree). ${ }^{17}$ The internal reliability was high, $\alpha=.86$.

Adults responded on a 7-point Likert scale to the 15-item Temporal Satisfaction with Life Scale (Pavot, et al., 1998) that also measures global satisfaction by combining judgements about past (5 items), present (5 items), and future (5 items) satisfaction; for example, 'If I had my past to live over, I would change nothing', 'I would change nothing about my current life', and 'I expect my future life will be ideal for me' $(1=$ strongly disagree; 2 = disagree; 3 = slightly disagree; $4=$ neither agree or disagree; $5=$ slightly agree; $6=$ agree; $7=$ strongly agree) ${ }^{18}$ The internal reliability was high, $\alpha=.91$.

\footnotetext{
${ }^{17}$ See Appendix F for the Satisfaction with Life Scale.

${ }^{18}$ See Appendix G for the Temporal Satisfaction with Life Scale.
} 
Happiness. Both adolescents and adults responded on a 7-point Likert scale to the 4item Subjective Happiness scale (Lyubomirsky \& Lepper, 1999), measuring global subjective happiness; for example, 'In general, I consider myself:' ( 1 = not a very happy person to $7=\mathrm{a}$ very happy person). ${ }^{19}$ Lyubomirsky and Lepper argued that general happiness is not the same as the sum of one's recent positive emotions, nor is it the same as being satisfied with life. For example, someone can be a happy person, but only somewhat satisfied with life. Someone can also consider themselves to be a very happy person although their recent experiences of joy and pride, for example, have been minimal. The Subjective Happiness scale, however, has been positively and highly correlated with emotion scales, e.g., the Affect Balance scale (Bradburn, 1969), and conceptualised as closely related to the measure of positive affect (Lyubomirsky, King, et al., 2005; Lyubomirsky \& Lepper, 1999). Item 4 on the Subjective Happiness scale is reverse-coded and often yields sub-standard Cronbach's alphas when included. The internal reliability was high for both adolescents and adults after removing item $4, \alpha=.84$ and $\alpha=.90$ respectively, consistent with previous research.

Eudaimonic wellbeing. The adolescents and adults responded to questions about their eudaimonia on different measures. Adolescents responded to items about their psychological and social wellbeing, and adults responded to items about their orientation to meaning, all of which are considered proxies for eudaimonic wellbeing, although they are not identical.

Adolescents responded on a 6-point Likert scale to the 12-item version of Keyes' (2005b, 2006) Mental Health Shortform that measures the frequency of emotional (3 items), psychological (4 items), and social wellbeing ( 5 items). ${ }^{20}$ To compose a eudaimonic wellbeing measure, psychological and social wellbeing were combined and emotional wellbeing was omitted; for example, 'In the last month you felt: That you have warm and trusting relationships with others', 'That you had something important to contribute to society', and 'That people are basically good' $(1=$ never; 2 once or twice a week; $3=$ about once a week; $4=$ two or three times a week; $5=$ almost every day; $6=$ everyday). The internal reliability of eudaimonic wellbeing was high, $\alpha=.88$, consistent with previous research.

Adults completed the 18-item Orientations to Happiness scale (Peterson, et al., 2005) on a 5 -point Likert scale $(1=$ not like me at all; $2=$ a little like me; $3=$ somewhat like me; $4=$ mostly like me; $5=$ very much like me). ${ }^{21}$ This scale measures endorsement of three forms of

\footnotetext{
${ }^{19}$ See Appendix $\mathrm{H}$ for the Subjective Happiness scale.

${ }^{20}$ See Appendix I for the Mental Health Short Form.

${ }^{21}$ See Appendix J for the Orientations to Happiness scale.
} 
happiness: pleasure (6 items), meaning (6 items), and engagement (6 items). To compose a eudaimonic wellbeing measure, meaning was used while pleasure and engagement were omitted. Meaning items included, 'My life serves a higher purpose' and 'What I do matters to society'. Meaning had a high internal reliability, $\alpha=.84$, consistent with previous research.

\section{Results}

\section{Analytic Strategy}

To answer the first research question, the relative endorsement of dampening, low arousal, high arousal, and self-focus savoring by adolescents and adults was examined with a Friedman's ANOVA followed by Wilcoxon's signed-rank tests. To answer the third and fourth research questions whether savoring strategies uniquely relate to wellbeing and whether age influences the savoring-wellbeing relationship, a two-step approach using structural equation modelling with AMOS (Anderson \& Gerbing, 1988; Arbuckle, 2009) was employed. In the first step, measurement models tested the construct validity of the wellbeing models, but not the savoring models, as they were established in Chapter 2. In the second step, the structural models tested the relationships between savoring and wellbeing. The last analyses answered the fourth research question by exploring the potential moderating role each savoring strategy played on the relationship between hedonic and eudaimonic wellbeing. Satisfaction with life and happiness were combined to form hedonic wellbeing, while eudaimonic wellbeing was composed of psychological and social wellbeing for adolescents, and orientation toward meaning for adults. After conducting regressions to determine if interactions between savoring and wellbeing were present, I used Modgraph (Jose, 2008) to interpret the meaning of significant interaction effects.

\section{Non-parametric tests: Do adolescents and adults similarly rank their endorsement of savoring strategies?}

A Friedman's ANOVA indicated adolescents reported using savoring strategies to significantly different degrees $\left(\chi^{2}(3)=75.92, p<.001\right)$. Six Wilcoxon's signed-rank tests were conducted to test for significant differences in endorsement between pairs of individual savoring strategies. A Bonferroni correction was applied and all reported effects are tested with a $p<.008$ level of significance. In descending order, adolescents reported high arousal $(M d n=2.82)$, selffocus $(M d n=2.64)$, dampening $(M d n=2.39)$ and low arousal strategies $(M d n=2.15)$. The differences in each of these ranks were significant, except for the difference between dampening and low arousal strategies. Results are reported for the z scores and effect sizes $(r)$ in Table 3.1 and depicted in Figure 3.1. 
Table 3.1. Summary of Wilcoxon's Signed-Rank Results: Adolescent $(N=463)$ and Adult $(N=980)$

Rankings of Dampening, High Arousal, Low Arousal, and Self-focus Savoring.

\begin{tabular}{ccccc}
\hline Variable & \multicolumn{2}{c}{ Adolescents } & \multicolumn{3}{c}{ Adults } \\
\hline z & $r$ & $\tau$ & $r$ \\
\hline Damp-LA & $-2.39^{\text {ns }}$ & - & -17.70 & -.55 \\
Damp-HA & -5.95 & -.28 & -24.38 & -.78 \\
Damp-SF & -3.52 & -.16 & -24.18 & -.77 \\
LA-HA & -8.75 & -.41 & -21.62 & -.69 \\
LA-SF & -6.39 & -.29 & -18.77 & -.60 \\
HA-SF & -3.56 & -.17 & -6.18 & -.20 \\
\hline
\end{tabular}

Note. Damp $=$ Dampening, LA $=$ Low Arousal, HA = High Arousal, and SF $=$ Self-focus Savoring. All $z$-scores are significant at the Bonferroni adjusted level of $p<.008$, except Damp-LA; ${ }^{\text {ns }}=$ nonsignificant; $r=$ Pearson's correlation coefficient, estimate of effect size.

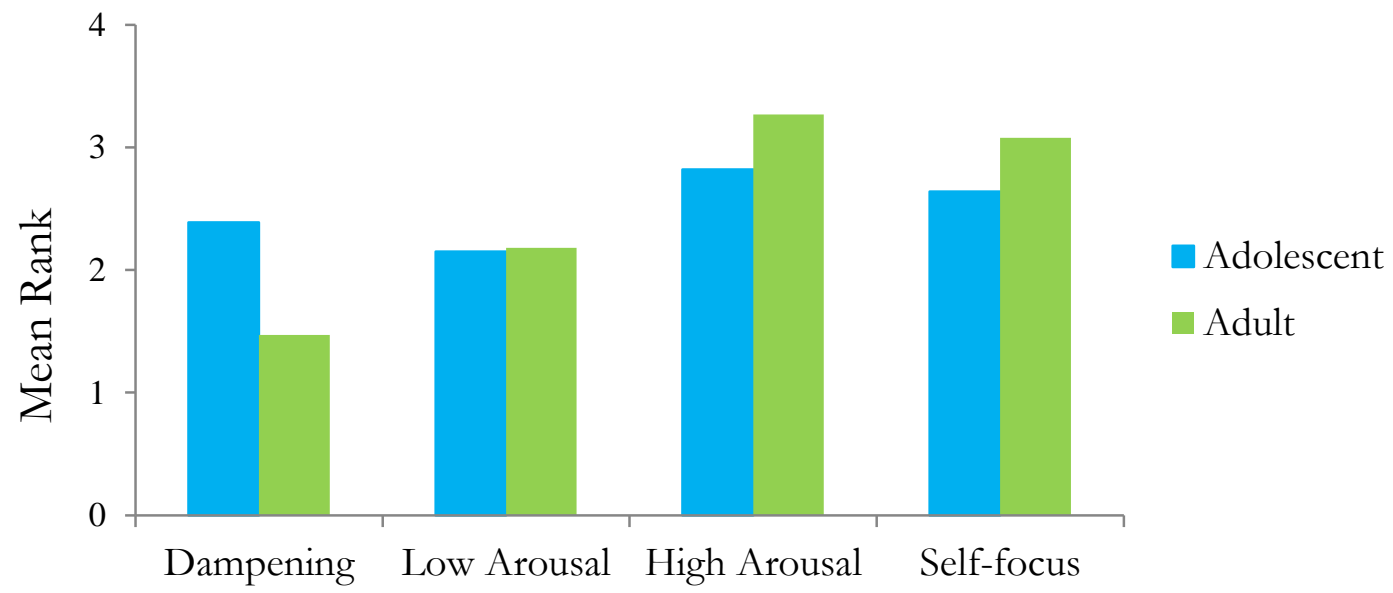

Figure 3.1. Adolescents' and adults' mean ranked endorsement for the four savoring strategies: dampening, low arousal, high arousal, and self-focus savoring.

A Friedman's ANOVA indicated adults also reported using savoring strategies to significantly different degrees $\left(\chi^{2}(3)=1317.80, p<.001\right)$. Six Wilcoxon's signed-rank tests were conducted to test for significant differences in endorsement between paired individual savoring strategies. A Bonferroni correction was applied and all reported effects met a $p<.008$ level of significance. In descending order, adults reported high arousal ( $M d n=3.27)$, self-focus ( $M d n=$ 3.08), low arousal $(M d n=2.18)$, and dampening savoring $(M d n=1.47)$. Each comparison in rank 
was significant. Results are reported for the $z$ scores and effect sizes in Table 3.1 and depicted in Figure 3.1

The differences in each of savoring ranks for adolescents were significant, except for the difference between dampening and low arousal strategies, consistent with Hypothesis 1. Also, supporting Hypothesis 1 was the finding that adolescents ranked differences between dampening and low arousal savoring and high arousal and self-focused savoring appear weaker than for adults, although no tests of significance between adolescents and adults were conducted. Finally, each comparison in rank for adults was significant, consistent with Hypothesis 2.

\section{Discriminant Validity: Do savoring strategies uniquely relate to wellbeing?}

A two step approach tested for the construct validity of the wellbeing model before testing the discriminant validity of the savoring model. By testing the construct validity of the wellbeing measurement model prior to testing the savoring and wellbeing structural model, it ensures that the structural model is based on an appropriate measurement model (Anderson \& Gerbing, 1988; Kline, 2005).

As with the previous chapter, the goodness of fit for each model was assessed using four criteria (Hu \& Bentler, 1999): the $\chi^{2} / \mathrm{df}$ ratio, the standardized version of Jöreskog and Sörbom's (1982) root mean square residual (sRMR), root mean square error of approximation (RMSEA) (Steiger, 1990), and the comparative fit index (CFI) (Bentler, 1990). Absolute fit was represented by sRMR and RMSEA, and incremental fit by CFI. Smaller values reflect better model fit for sRMR and RMSEA, whereas larger values reflect better model fit for CFI. Hu and Bentler suggest cut-off values of .08 for sRMR, .06 for RMSEA and .95 for CFI, with Bentler and Bonnett (1980) recommending CFI values above .90.

Measurement Models. The savoring measurement models reported in Chapter 2 constituted the starting point of this process, where savoring of everyday positive events was represented by: dampening, low arousal, high arousal and self-focus savoring for both adolescents $\left(\chi^{2} / \mathrm{df}=2.55, \mathrm{CFI}=.93, \mathrm{RMSEA}=.06, \mathrm{sRMR}=.04\right)$ and adults $\left(\chi^{2} / \mathrm{df}=4.16, \mathrm{CFI}\right.$ $=.94, \mathrm{RMSEA}=.06, \mathrm{sRMR}=.04)$. Then, an adolescent and adult measurement model of satisfaction, happiness, and eudaimonic wellbeing was tested prior to testing the path model. Parcels were constructed for the satisfaction and eudaimonic latent constructs since both scales had more items than the suggested three indicators for each latent variable (Kline, 2005). Since there were only three items for the happiness scale, the individual items were the indicators.

Parcelling reduces the complexity of the model by reducing the amount of redundant error, which reduces the likelihood of spurious correlations (Little, et al., 2002). When scales are 
multidimensional, there are several parcelling techniques to choose from, but when scales are unidimensional, the process is straightforward. For the multidimensional scales I chose to parcel items following their established facet structure, called the internal consistency approach (Kishton \& Widaman, 1994). Consider a 9-item scale, with three items in each of three facets: facet A, B, and C. With an internal consistency approach, parcels include the average or sum of all items within one facet: $A_{1}+A_{2}+A_{3}$ compose parcel 1, $B_{1}+B_{2}+B_{3}$ compose parcel 2, and $\mathrm{C}_{1}+\mathrm{C}_{2}+\mathrm{C}_{3}$ compose parcel 3. The internal consistency approach has several benefits, two of which are that it attempts to keep the multidimensional nature of the scale explicit, and allow for the unique variance of each facet to relate to other constructs in the model (Hoyle \& Smith, 1994). This multidimensional approach was applied to the adult life satisfaction scale and adolescent eudaimonic wellbeing scale. And although the adult eudaimonic wellbeing scale was unidimensional, I parcelled items based on their conceptual similarities. On the other hand, the unidimensional satisfaction measure reported by adolescents was straightforward; items were grouped in numerical order since there was no multidimensional structure to preserve.

For adolescents, the satisfaction scale included items 1 and 2 in parcel 1, items 2 and 3 in parcel 2, and items 4 and 5 in parcel 3 (Appendix F). The eudaimonia scale included items 4 thru 6 in parcel 1 (psychological wellbeing), items 7 thru 9 in parcel 2 (belonging), and items 10 thru 12 in parcel 3 (faith in people and society) (Appendix I). For adults, the satisfaction scale included items 1 thru 5 in parcel 1 (satisfaction with the past), items 6 thru 10 in parcel 2 (satisfaction with the present), and items 11 thru 15 in parcel 3 (satisfaction with the future) (Appendix G). The eudaimonia scale included items 5 and 11 in parcel 1 (sense of responsibility), items 12 and 17 in parcel 2 (sense of meaning), and items 2 and 14 in parcel 3 (sense of purpose) (Appendix J).

The adolescent and adult measurement models had a sufficient participant to parameter ratio (13.94 and 29.70 respectively) with three latent variables that were allowed to correlate: satisfaction, happiness, and eudaimonia. As seen in Figure 3.2, results of the measurement models indicated that each parcel loaded highly on the corresponding latent factor. Both models 
Model A) Adolescent Measurement Model

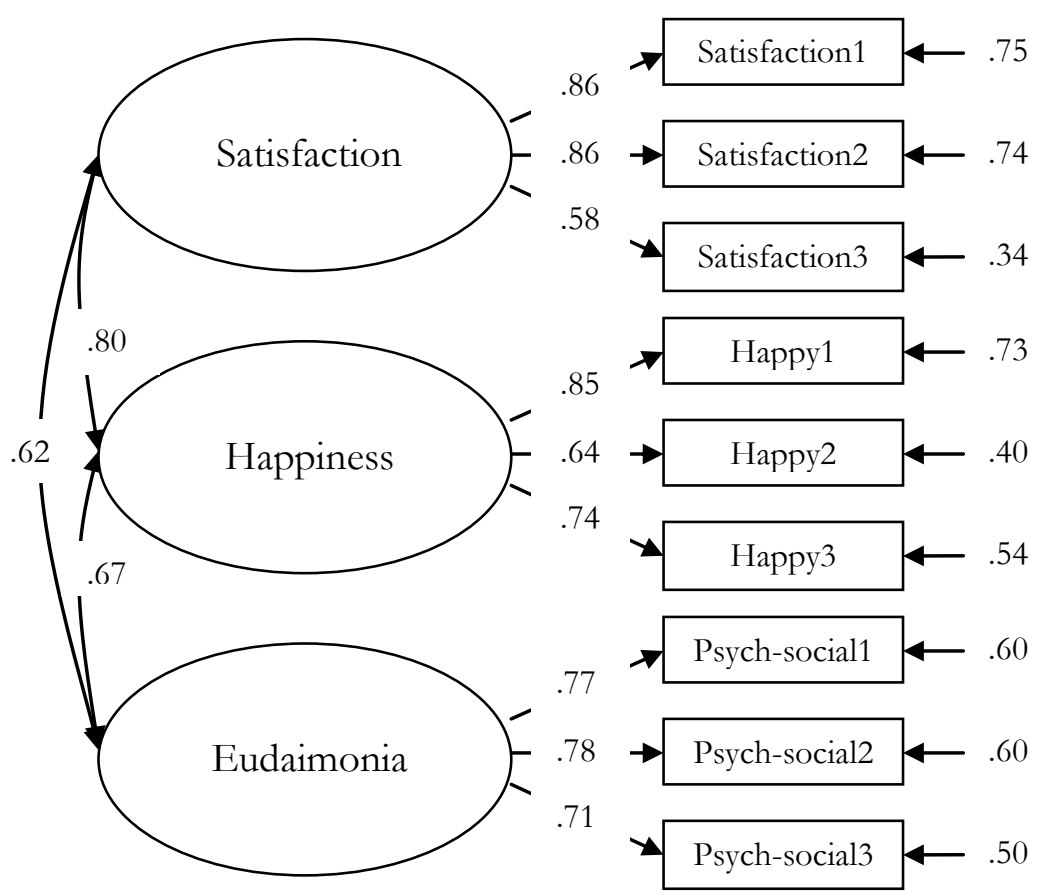

Model B) Adult Measurement Model

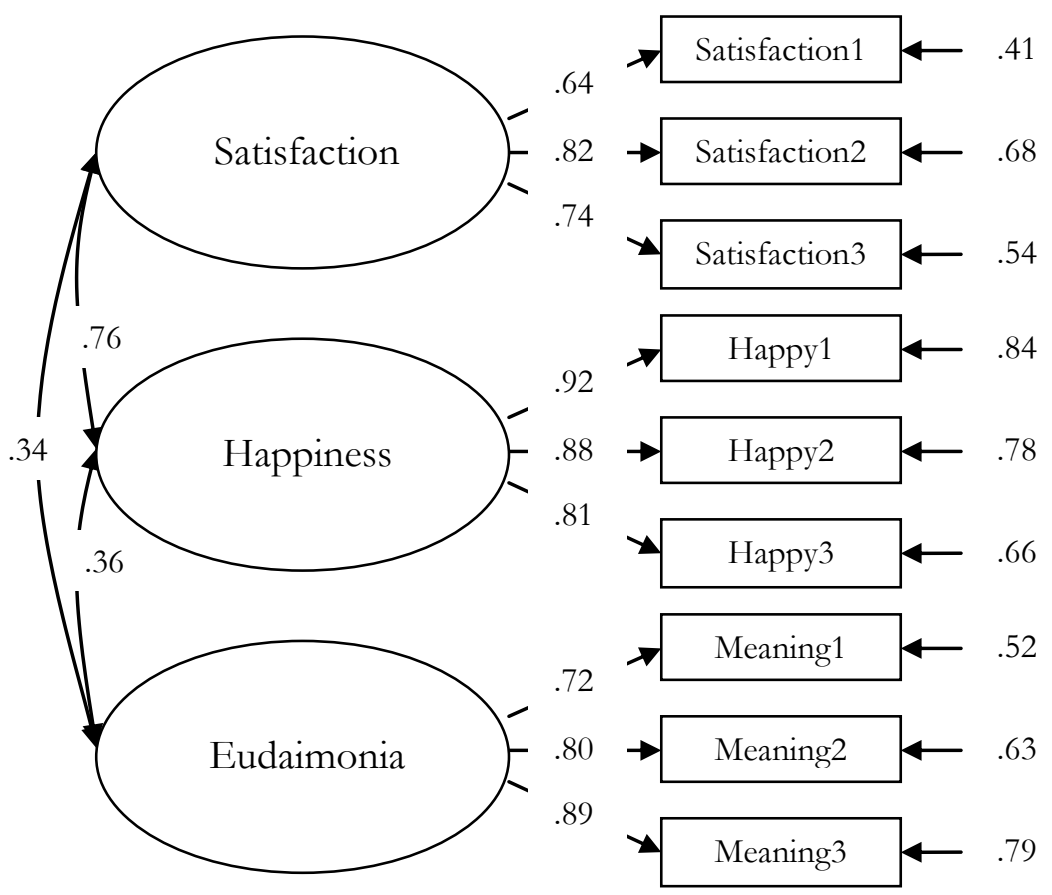

Figure 3.2. Standardised adolescent (Model A, $N=463$ ) and adult (Model B, $N=980$ ) measurement models of satisfaction, happiness and eudaimonia. 
fit the data well according to model-fit indices, apart from the chi square ratio statistic and root mean square error of approximation, which indicated an adequate model fit. The adolescent $\left(\chi^{2} / \mathrm{df}=3.84, \mathrm{CFI}=.94, \mathrm{RMSEA}=.05, \mathrm{sRMR}=.04\right)$ and adult $\left(\chi^{2} / \mathrm{df}=4.09, \mathrm{CFI}=.98\right.$, RMSEA $=.06$, sRMR $=.04)$ models' latent variable correlations, betas, and squared multiple correlations are reported in Figure 3.2.

Structural Model. Two latent path models were designed to investigate the construct validity of the four factor model of savoring everyday positive events, one for adolescents and one for adults. Dampening, low arousal, high arousal, and self-focus savoring were allowed to predict life satisfaction, happiness, and eudaimonic wellbeing. The initial exploratory models included these seven latent variables (four savoring variables and three wellbeing indicators) and allowed each latent savoring factor to predict each latent positive health indicator (i.e., a fully saturated model). In total, there were 12 structural pathways for each base model.

Results of these two base models identified several significant predictors. Five significant predictors were found for adolescents: low arousal savoring to happiness $(\beta=-.41, p<.05)$; high arousal savoring to happiness $(\beta=.45, p<.01)$; and self-focus savoring to satisfaction $(\beta=.84$, $p<.01)$, happiness $(\beta=.47, p<.05)$, and eudaimonic wellbeing $(\beta=.73, p<.01)$. Six significant predictors were found for adults: dampening to satisfaction $(\beta=-.20, p<.001)$, happiness $(\beta=$ $.32, p<.001)$, and eudaimonic wellbeing $(\beta=-.20, p<.001)$; low arousal to eudaimonic wellbeing $(\beta=.33, p<.01)$; and self-focus to satisfaction $(\beta=.56, p<.05)$, and happiness $(\beta=$ $.62, p<.01)$. Both the adolescent base model $\left(\chi^{2} / \mathrm{df}=2.20, \mathrm{CFI}=.94\right.$, RMSEA $=.05$, sRMR $=$ $.05)$ and adult base model $\left(\chi^{2} / \mathrm{df}=2.79, \mathrm{CFI}=.96, \mathrm{RMSEA}=.04, \mathrm{sRMR}=.04\right)$ fit the data well.

Next, the base models were pruned by removing each non-significant path individually, starting with the least significant and ending when only significant relationships remained. At each stage of the pruning process, the beta weights, phis, and error terms were assessed to ensure multicollinearity was not a significant problem. The pruning of the adolescent base model took six steps, and identified one new path (dampening savoring to satisfaction; $\beta=-.30, p<.001$ ). The pruning of the adult base model also took six steps, but no new paths were identified. Two paths, however, were altered post-hoc to test whether the adult model paralleled the high arousal to happiness relationship found with adolescents. As seen in Figure 3.3, a path from high arousal to happiness was added, and a path from self-focus-savoring to happiness was removed, which 
identified a similar relationship from adult high arousal savoring to happiness $(\beta=.39, p<.001)$ as adolescents.

- - - Negative Relationship

- Positive Relationship
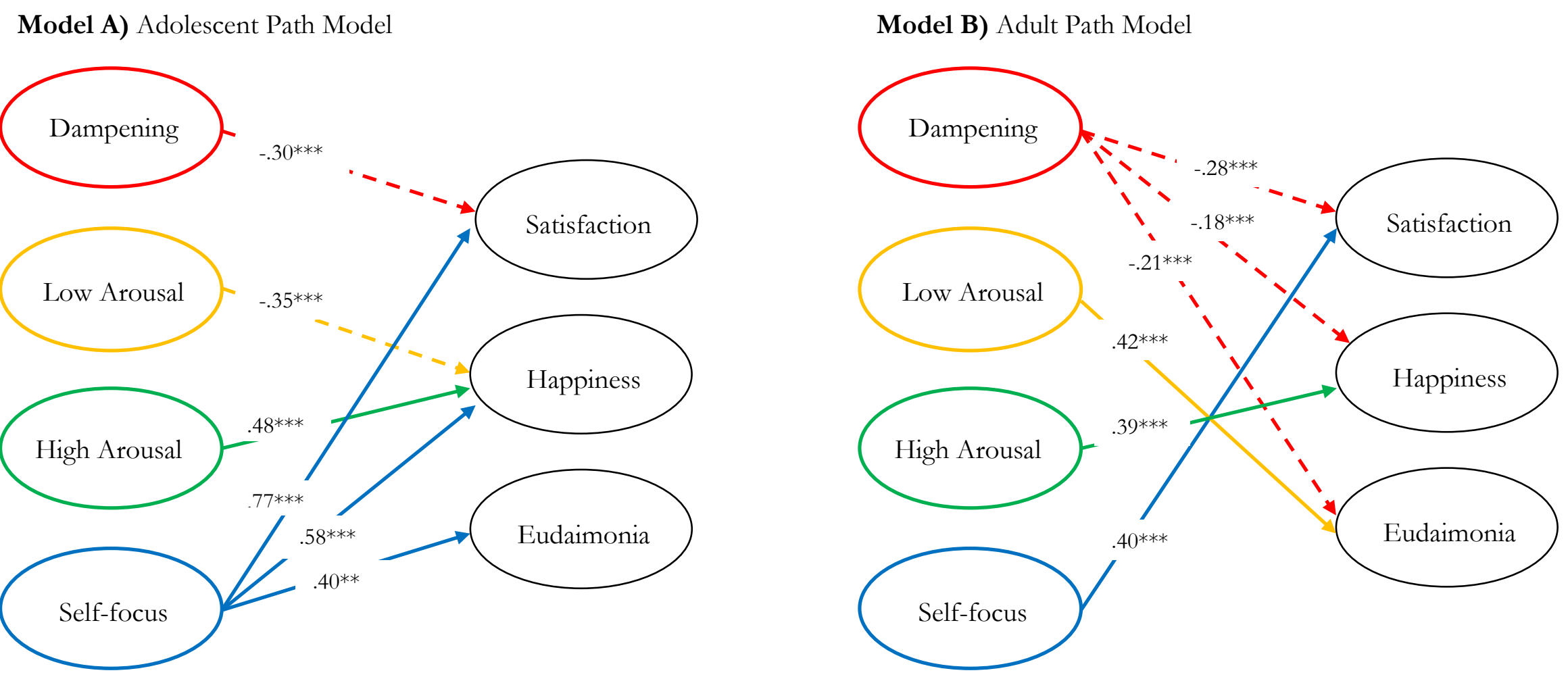

Figure 3.3. Standardised path models of adolescent (Model A, $N=463$ ) and adult (Model $\mathrm{B}, N=980$ ) everyday savoring strategies and their relationship to satisfaction, happiness, and eudaimonia. Note. ${ }^{* *} p<.01 .{ }^{* * *} p<.001$. 
The final pruned adolescent model displayed good model fit: $\chi^{2} / \mathrm{df}=2.15$, CFI $=.94$, RMSEA $=.05$, sRMR $=.05$, and the final pruned adult model displayed good model fit: $\chi^{2} / \mathrm{df}=$ $2.82, \mathrm{CFI}=.96, \mathrm{RMSEA}=.06, \mathrm{sRMR}=.04$. The models supported all the predicted shared and disparate relationships between savoring, and satisfaction, happiness, and eudaimonia for adolescents and adults (Hypotheses 3 through 6); however, several significant unpredicted findings were also present.

For the most part, the four savoring strategies differentially predicted satisfaction, happiness, and eudaimonia for both adolescents and adults, supporting the unique predictive ability of the four savoring strategies. As expected, however, adolescents' savoring was related to multiple wellbeing indicators. In particular, self-focus savoring predicted increases in all three wellbeing indicators for adolescents, while adult self-focus savoring only predicted satisfaction with life. Dampening for adults, however, negatively predicted all three wellbeing indicators, for adolescents it only predicted decreases in satisfaction.

Reviewing the findings from the path models revealed that Hypothesis 3 was confirmed; dampening savoring related to a decrease in life satisfaction, in that dampening savoring was moderately and negatively associated with life satisfaction in both groups (adolescents, $\beta=-.30$, $p<.001$; adults, $\beta=-.28, p<.001)$. A finding that was not hypothesised, although not unexpected, was that adult dampening savoring was negatively associated with happiness $(\beta=$ $.18, p<.001)$, and meaning $(\beta=-.21, p<.001)$. Altogether, adult dampening savoring predicted a decrease in all three wellbeing indicators, while adolescent dampening savoring only predicted a decrease in one wellbeing indicator.

Supporting Hypothesis 4, high arousal was positively associated with happiness for adolescents $(\beta=.48, p<.001)$ and adults $(\beta=.39, p<.001)$. Adolescent self-focus savoring was an important predictor of wellbeing. It was positively associated with all three styles of wellbeing (although only the relationship with life satisfaction was specifically predicted): life satisfaction ( $\beta$ $=.77, p<.001)$, happiness $(\beta=.40, p<.01)$, and eudaimonia $(\beta=.58, p<.001)$. As predicted by Hypothesis 4 , adult self-focus strategies were positively associated with life satisfaction $(\beta=$ $.40, p<.001)$. Overall, both samples' high arousal savoring positively predicted happiness, and both samples' self-focus savoring positively predicted life satisfaction, however, self-focus savoring also predicted happiness and eudaimonia for adolescents only.

The last two hypotheses-Hypotheses 5 and 6-were supported by the finding that low arousal was negatively associated with happiness for adolescents $(\beta=-.35, p<.001)$, but 
positively associated with meaning for adults $(\beta=.42, p<.001)$. As expected by Hypothesis 5 , adolescents' low arousal and dampening savoring both manifested a negative relationship with wellbeing indicators. Lastly, adults low arousal savoring positively predicted eudaimonic wellbeing as expected by Hypothesis 6. Refer to Figure 3.3 for a side-by-side view of the two age-based models.

\section{Do savoring strategies moderate the relationship from hedonic to eudaimonic wellbeing?}

The independent variable was hedonic wellbeing, which was a combination of satisfaction with life and happiness. The moderators were each of the four savoring strategies: dampening, low arousal, high arousal, and self-focus savoring. Lastly, the dependent variable was eudaimonic wellbeing (i.e. psychological and social wellbeing for adolescent, and orientation toward meaning for adults).

A moderating relationship is one where the relation between a predictor variable and an outcome varies as a function of different levels of another predictor variable (the moderator) (Baron \& Kenny, 1986). A moderator variable can either buffer or exacerbate a negative impact on the outcome, or diminish or enhance a positive impact on the outcome; for example, Hypothesis 7 predicts that adolescents' low arousal savoring would diminish the impact of hedonic wellbeing on eudaimonic wellbeing. On the other hand, Hypothesis 8 predicts that adults' low arousal savoring would enhance the impact of their hedonia on eudaimonia, which would be supported if it is found that hedonia was more highly related to eudaimonia for individuals who were engaged in low arousal savoring.

I conducted a total of 8 moderations ( 4 with each sample) to test the moderation of the relationship between hedonic and eudaimonic wellbeing by the four savoring strategies. To reduce multicollinearity, all predictor variables were centred before creating the interaction terms (Howell, 2009). The main effects of the predictor and moderator were entered in step 1 (e.g. hedonic wellbeing and low arousal savoring) and the interaction was entered in step 2 (e.g. hedonic wellbeing $\mathrm{x}$ low arousal savoring).

As seen in Table 3.2, a total of three significant savoring moderations were found, one for adolescents (Regression 1) and two for adults (Regression 2 and 3; one of which was marginally significant: the moderation of hedonic and eudaimonic wellbeing by low arousal savoring $(p=.06))$. Adolescents' low arousal savoring diminished the positive impact of hedonic 
Table 3.2. The Impact of Hedonic Wellbeing on Eudaimonic Wellbeing, moderated by Low Arousal Savoring, and High Arousal Savoring.

\begin{tabular}{|c|c|c|c|c|c|}
\hline \multirow[b]{2}{*}{ Variable } & \multicolumn{5}{|c|}{ Regression 1) Adolescents $(N=463)$} \\
\hline & $B$ & SE B & $\beta$ & $\Delta R^{2}$ & $\operatorname{Sign} F \Delta$ \\
\hline Step 1 & & & & .36 & $<.001$ \\
\hline Hedonic WB & .42 & .03 & $.55^{* * *}$ & & \\
\hline Low Arousal & .10 & .03 & $.16^{* * *}$ & & \\
\hline Step 2 & & & & .01 & .007 \\
\hline \multirow[t]{2}{*}{ HedxLA } & -.05 & .02 & $-.10^{* *}$ & & \\
\hline & \multicolumn{3}{|c|}{ Regression 2) Adults $(N=980)$} & & \\
\hline Variable & $B$ & SE B & $\beta$ & $\Delta R^{2}$ & $\operatorname{Sign} F \Delta$ \\
\hline Step 1 & & & & .14 & $<.001$ \\
\hline Hedonic WB & .25 & .03 & $.29 * * *$ & & \\
\hline Low Arousal & .24 & .02 & $.20^{* * *}$ & & \\
\hline Step 2 & & & & .003 & .056 \\
\hline \multirow[t]{2}{*}{ HedxLA } & .04 & .02 & $.06^{\dagger}$ & & \\
\hline & \multicolumn{3}{|c|}{ Regression 3) Adults $(N=980)$} & & \\
\hline Variable & $B$ & SE B & $\beta$ & $\Delta R^{2}$ & $\operatorname{Sign} F \Delta$ \\
\hline Step 1 & & & & .12 & $<.001$ \\
\hline Hedonic WB & .24 & .03 & $.27 * * *$ & & \\
\hline High Arousal & .12 & .03 & $.15^{* * *}$ & & \\
\hline Step 2 & & & & .01 & .018 \\
\hline HedxHA & .05 & .02 & $.07 *$ & & \\
\hline
\end{tabular}

wellbeing on eudaimonia, supporting Hypothesis 7 (Regression 1; Figure 3.2); however, contrary to Hypothesis 7, dampening savoring was not found to be a moderator of the wellbeing relationship. A similar non-significant finding was found for adult dampening savoring, contradicting Hypothesis 8. Hypothesis 9 was also unsupported by finding that neither adolescents' high arousal nor self-focused savoring was a moderator of the hedonic eudaimonic relationship. The last hypothesis, Hypothesis 10, was partially supported. Adults' low arousal and high arousal savoring enhanced the positive impact of hedonic wellbeing on eudaimonic wellbeing (Regression 2 and 3 respectively; Figure 3.2), but self-focused savoring did not.

Figure 3.4 plots the significant savoring moderators of hedonia and eudaimonia. Low 

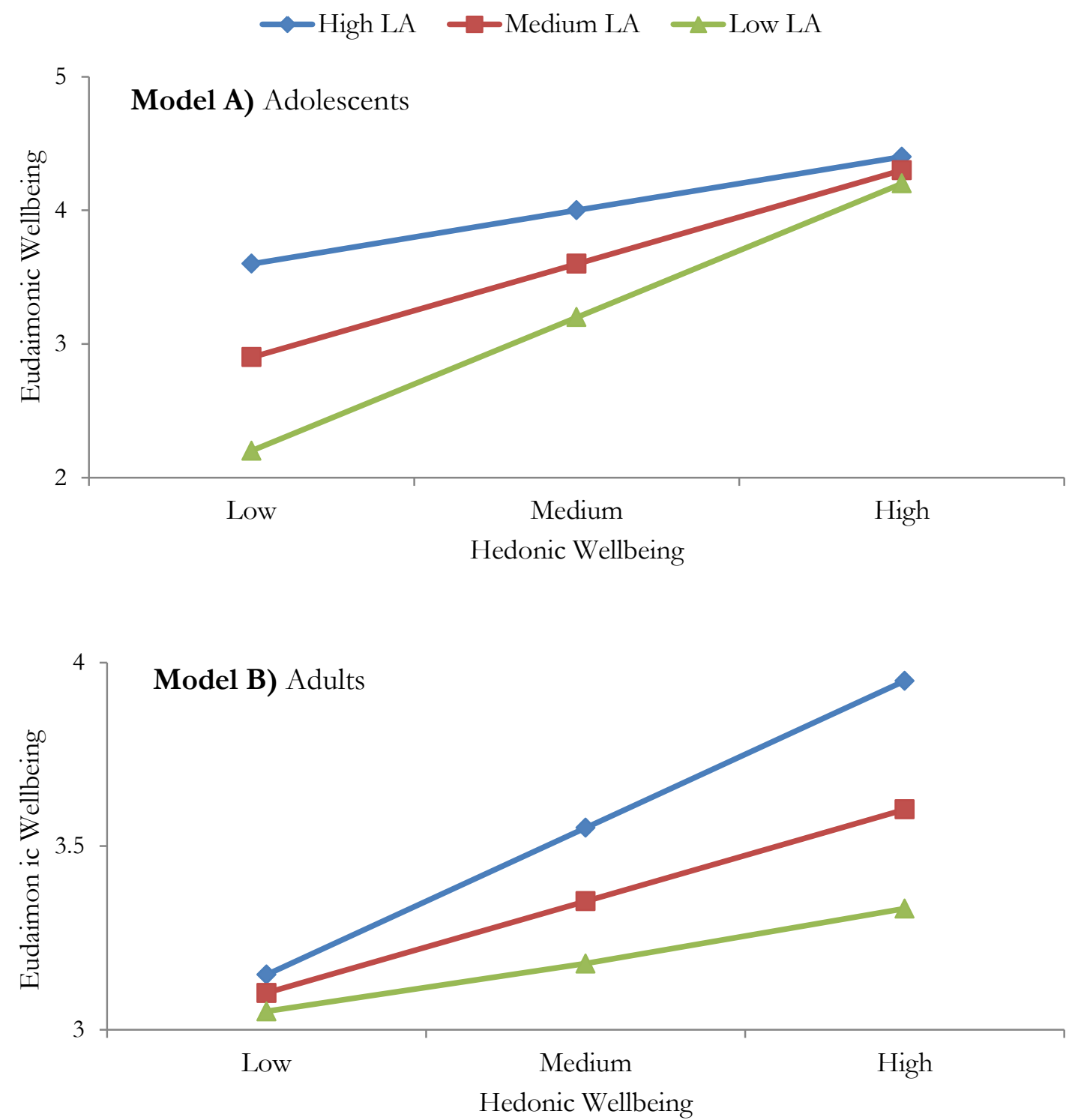

Figure 3.4. Low arousal savoring moderating the impact of hedonic wellbeing on eudaimonic wellbeing for adolescents (Model A) and adults (Model B).

arousal savoring was a significant main predictor of eudaimonia for adolescents, indicating that low arousal savoring positively predicted eudaimonia, a finding that was absent in the previous structural model. In the structural model reported above, adolescents' low arousal savoring did not evidence a relationship with eudaimonia. The adolescent moderation finding indicated that low arousal savoring had a diminishing affect on the relationship from hedonic to eudaimonic wellbeing. In effect, the strongest relationship between hedonia and eudaimonia occurred with individuals low in low arousal savoring. However, the three low arousal group means were most 
different under conditions of low hedonia, indicating that increases in low arousal savoring enhances the impact of hedonia on eudaimonia under conditions of low hedonic wellbeing; in other words, high low arousal savoring contributed to higher mean levels of eudaimonia when hedonia was low. Figure 3.4 (Model A) plots the effect of high, medium, and low levels of low arousal savoring on the relationship between hedonic and eudaimonic wellbeing for adolescents.

Like adolescents, adults' low arousal savoring was a significant (marginally) main predictor of eudaimonia, which was also found in the previous adult structural model. Unlike the adolescent moderation model, however, adults' low arousal savoring enhanced the impact of hedonic wellbeing on eudaimonic wellbeing as predicted by Hypothesis 10. Figure 3.4 (Model B) shows that with high adult low arousal savoring, increases in hedonic wellbeing were associated with more dramatic increases in eudaimonic wellbeing compared with low levels of low arousal savoring where increases in hedonic wellbeing were associated with more mild increases in eudaimonic wellbeing.

Although, Hypothesis 10 was not supported by the finding that self-focused savoring was not a significant moderator of the wellbeing relationship, it was supported by the finding that adult high arousal savoring had the same impact on hedonic and eudaimonic wellbeing as did low arousal savoring, i.e. it is also an enhancer. High arousal savoring was a main predictor of eudaimonia (Table 3.2), and it increased the impact of hedonic wellbeing on eudaimonic wellbeing under conditions of increasing hedonic wellbeing. Model B in Figure 3.4 is identical to the pattern for high arousal savoring.

\section{Discussion}

This study first examined how adolescents and adults ranked their dampening, low arousal, high arousal, and self-focused everyday savoring strategies. Then it examined the interrelationships among these four savoring strategies and life satisfaction (cognitive wellbeing), happiness (emotional wellbeing), and eudaimonia (functional wellbeing) in adolescent and adult samples. Four major research questions structured the current study: (1) Do adolescents and adults similarly rank their endorsement of savoring strategies (2) Do savoring strategies uniquely relate to wellbeing (3) Does age appear to influence the savoring and wellbeing relationships, and (4) Do savoring strategies moderate the relationship from hedonic to eudaimonic wellbeing? The findings for each of these questions will be discussed in turn.

\section{Hypotheses 1 and 2: Ranking of the four everyday savoring strategies}

As predicted, adolescents and adults ranked their strategies similarly, with the exception of dampening and low arousal savoring, which were similarly ranked by adolescents but 
differentially ranked by adults. Both groups indicated the same preference for their first and second place savoring strategies: high arousal savoring, and then self-focused savoring. Adolescents' low arousal and dampening savoring tied for third place. Low arousal savoring was third place for adults, and dampening was their least endorsed savoring strategy. Although there were more similarities than differences, the effect sizes for the rankings were generally larger for adults than adolescents, indicating that adults had clearer preferences for which savoring strategies they endorsed in their everyday positive experiences. These findings support the view that adolescence is an important time for savoring development. The ability to differentiate strategies is on its way to being well-formed in adolescence, and by adulthood, these preferences appear distinct (Bryant, et al., 2011; Bryant \& Veroff, 2007).

\section{Hypotheses 3 and 4: Savoring strategies: Unique predictors of wellbeing}

The results from structural modelling supported the prediction that each of the dampening, low arousal, high arousal, and self-focused savoring strategies would uniquely predict wellbeing. The hypothesis that dampening savoring would negatively predict satisfaction with life was supported, confirming previous research by Quoidbach et al. (2010) that finding fault with a positive event or mentally travelling to a negative version of the future, for example, predicted a decrease in satisfaction with life. The results of Quoidbach and colleagues' study of 82 university students and employees who responded to questions about their satisfaction with life, was replicated with the present study of 1,443 community adolescents and adults. It confirmed their finding that dampening savoring would negatively predict positive affect and that low arousal savoring would positively predict wellbeing, although not positive affect for adults (Quoidbach, et al.). Unlike Quoidbach et al., this study was unable to find the weak link between low arousal savoring and positive affect that they found for adults. Instead, the current study found that absorbing the moment and blocking outside distractions (i.e. low arousal savoring) positively and strongly predicted eudaimonic wellbeing for adults.

The inclusion of eudaimonic wellbeing and the use of more robust and conservative analyses (i.e. latent path modelling) are arguable reasons why the present study was unable to support the previously identified weak positive link between positive affect and low arousal savoring. I chose to include eudaimonic wellbeing because it is a valuable indication of positive functioning and mental health (Keyes, 2005a, 2007), and I chose a sophisticated strategy to analysing savoring and wellbeing to more accurately represent the dynamic interaction of these phenomenon. Although there is no savoring research to make comparisons to, the connection 
between low arousal savoring and eudaimonic wellbeing found here and results from related fields support this association, which will be discussed further in the next subsection.

Results supported the fourth hypothesis that high arousal and self-focused savoring would predict increases in wellbeing. Specifically, self-focused savoring (i.e. counting blessings and congratulating one's self) positively predicted life satisfaction, and high arousal savoring (i.e. sharing with others and behavioural expression) positively predicted happiness. Interestingly, previous savoring research was unable to find the association between high arousal savoring strategies and frequent positive affect (Quoidbach, et al., 2010), although several studies support the association between sharing with others and behavioural expression, and happiness and enjoyment (Bryant \& Veroff, 2007; Gable, et al., 2004; Langston, 1994; Reis, et al., 2010). The link between self-focused savoring and satisfaction with life parallels the extensive research support for the consistent link between counting blessings, i.e. gratitude, and life satisfaction from both observational and experimental methods (see Wood, Froh, \& Geraghty, 2010).

Taken together, the style of savoring that predicted decreases in wellbeing was not the same style of savoring that predicted increases in wellbeing. Different savoring strategies positively predicted happiness, satisfaction, and, as I discuss in the following subsection, eudaimonia, supporting the independent utility of the four everyday savoring strategies.

\section{Hypotheses 5 and 6: Savoring predictors and wellbeing and the influence of age}

Hypotheses 5 and 6 were investigated with separate structural models. Side-by-side comparisons of the adolescent and adult models of everyday savoring and wellbeing were made, rather than using equality constraints, because of the inconsistency of measures between the two samples (see Figure 3.3). Both Hypotheses 5 and 6 were confirmed. Adolescents' dampening and low arousal savoring negatively predicted cognitive and emotional wellbeing respectively. Adults' low arousal savoring positively predicted eudaimonic wellbeing. The fact that adolescents' use of both dampening and low arousal savoring was negatively predictive of wellbeing confirms the strong link found between these two savoring strategies found in Chapter 2; their link is further affirmed by their similar quality of relationship with wellbeing-both negative.

Previous research explains these differences in adolescent and adult low arousal and wellbeing findings by suggesting adolescents are contra-hedonic (motivated to decrease positive emotions), while adults are pro-hedonic (motivated to maintain or increase positive emotions) (Riediger, et al., 2009). Expanding this support, research indicates that over the life-span, the definition of happiness changes from excitement to peacefulness, and peacefulness appears to be a construct that is positively aligned or overlapping with eudaimonia (Kamvar, et al., 2009; 
Mogilner, et al., 2011). The present study offers evidence that low arousal savoring would be a mechanism that relates to increases in this calmer form of happiness during adulthood. Confirmation for the expected strong positive link between adult low arousal savoring and eudaimonia was also evidenced by the finding that present focus appears to be heightened in older age, making later life emotionally meaningful (Carstensen, 2006; Carstensen, Fung, \& Charles, 2003; Carstensen, et al., 2010). By absorbing the moment and blocking out distractions, adults arguably facilitate or maintain present focus, and, as found here, eudaimonic wellbeing. Conversely, adolescents do not prefer a calming savoring strategy to increase wellbeing, and from this study it appears these calmer savoring strategies interfere with adolescent wellbeing.

Several interesting findings that were not hypothesised were found: adolescent selffocused savoring positively predicted all three wellbeing types, and adult dampening savoring negatively predicted all three wellbeing types. Self-focused savoring had a stronger relationship with cognitive, emotional, and eudaimonic wellbeing than any of the other three savoring strategies for adolescents, although their top ranked strategy was high arousal savoring (see above). Bryant and Veroff's (2007) work with university students showed that sharing with others-a component of high arousal savoring — was the greatest predictor of enjoyment. This study found that for everyday positive life events, however, it was the second ranked strategy, self-focused savoring, that was the most powerful positive predictor of wellbeing. Past and recent research on counting blessings and gratitude confirms these are important processes for encouraging adolescent wellbeing (Froh \& Bono, 2011; Froh, Emmons, Card, Bono, \& Wilson, in press; Froh, et al., 2008; Froh, Yurkewicz, \& Kashdan, 2009).

In sum, some specific forms of savoring appeared to have similar relationships to wellbeing across adolescents and adults (e.g. high arousal savoring), whereas other strategies had markedly different effects on wellbeing (e.g. low arousal savoring). The variable impact of savoring on particular components of wellbeing support using specific strategies to influence specific wellbeing components, and that the ability to respond to everyday positive life events with a variety of savoring strategies will beneficially contribute to overall wellbeing. Many selfhelp books, workshops, and training programs focus on specific techniques (e.g. practising mindfulness or engaging in flow) to increase wellbeing; the findings here suggest facilitating multiple techniques for the greatest positive and holistic everyday wellbeing. In addition, adolescent strengths may be different than adult strengths. If an aim of intervention was to work from pre-existing strengths, then it would be important to consider encouraging self-focused strategies rather than low arousal strategies in adolescents, for example. Recent research confirms 
this notion of fit, supporting an appropriate fit between wellbeing enhancing strategies and group characteristics (Boehm \& Lyubomirsky, 2009; Lyubomirsky, 2008; Lyubomirsky, Sheldon, et al., 2005). Workshops or intervention cost-effectiveness is also important to consider, especially for schools; knowing which strategies might encourage the greatest change for the least amount of monetary investment might facilitate schools adopting skills training for positive adolescent development.

\section{Hypotheses 7 through 10: Moderating the relationship from hedonic to eudaimonic wellbeing}

After investigating the unique predictive ability of the four savoring strategies, their potential to moderate the relationship between hedonic (i.e. satisfaction with life and happiness) and eudaimonic (i.e. psychological and social, and meaning) wellbeing was tested. No previous study, to my knowledge, has examined savoring as a moderator of the hedonic-eudaimonic relationship, although research supports the important influence feeling good has on functioning well (e.g., Fredrickson, 2005b). This was the first study, to my knowledge, to examine how everyday savoring impacts the ability of feeling good to improve functioning well.

One savoring strategy moderated wellbeing for adolescents and two savoring strategies moderated wellbeing for adults. Low arousal savoring was a moderator for both samples, but with different effects. Adolescent low arousal savoring had a diminishing impact on hedonia's ability to increase eudaimonia, partially confirming Hypothesis 7. In contrast, adult low arousal savoring enhanced the effect of hedonia on eudaimonia, supporting Hypothesis 10. High arousal savoring also had an enhancing impact on wellbeing for adults, supporting Hypothesis 10. Several predictions were not supported by the evidence: dampening savoring and self-focused savoring did not moderate the wellbeing relationship for either sample, and neither did adolescent high arousal savoring.

In conclusion, the multiple analyses undertaken in this chapter suggest that the fourfactor model of everyday savoring is meaningful (valid) and psychometrically robust. The findings suggest that a relative preference for savoring strategies does not mean that the top ranked strategy will be the most powerful predictor of wellbeing. The four everyday savoring strategies each predicted specific types of wellbeing, which supports using several strategies for living the good life and specific strategies to bolster specific wellbeing needs. Caution must be taken in interpreting the several intriguing differences between adolescent and adult savoring and wellbeing. As noted before, the adolescent and adult samples may be different, and some measures were not identical across samples. However, despite these differences, broad 
similarities in findings suggest that general comparisons can be made here. Nevertheless, apparent differences might be due to age (the preferred interpretation) or to sample differences (a less interesting interpretation). Regardless, future investigations into the developmental influences on savoring and wellbeing are merited, and hopefully the presently identified findings can suggest hypotheses for this further work. The next chapter turns to observing adolescents' savoring and wellbeing over time. 


\section{Chapter 4}

\section{Adolescent Savoring and Wellbeing over Time}

This chapter reports research that for the first time, to my knowledge, has examined the causeand-effect relationships between savoring and wellbeing over time with adolescents. The previous chapter documented several interesting and interpretable concurrent relationships between adolescent savoring and wellbeing. It showed that although low arousal savoring is conceptualised as a set of strategies aimed at improving positive emotions, for adolescents it seemed that it decreased their happiness. Chapter 3 also reported results that adolescents who are higher in low arousal savoring dampen the beneficial impact of satisfaction and happiness on their psychological and social wellbeing. On the positive side, adolescents who use high arousal and self-focus savoring experience more satisfaction, happiness, and eudaimonia. As with all correlational research, however, the associations that were found do not support cause-andeffect interpretations.

The current chapter expands on the previous findings by observing the relationships between savoring and hedonic and eudaimonic wellbeing over time, and by doing so, it presents evidence that suggest cause-and-effect relationships. It begins by first investigating the fourfactor structure of savoring at the second time-point, the test-retest reliabilities of the four factors of savoring everyday positive events, and then examines the interrelationships among these savoring strategies over time. For savoring to be a reliable structure, each strategy must evidence reasonable stability over time. By also investigating the interrelationships among savoring types, I am testing the associations of savoring strategies over time, and expecting that the same savoring strategy at Time 1 will be the greatest predictor of itself at Time 2 . Since the CFA at Time 1 suggests considerable interrelationships among the four savoring types (Chapter 2: Figure 2.1), an examination of longitudinal interrelationships will be more stringent because of the residualised structure of the path model.

After the validity and reliability of savoring are examined, predictions from Chapter 3 are extended by testing the impact of each savoring strategy on positive emotion (hedonic wellbeing), and psychological and social wellbeing (eudaimonic wellbeing) over one month. Then, a simplified model tests whether savoring predicts positive affect while positive affect predicts savoring, or as the broaden-and-build theory might suggest: savoring and positive affect affect each other in a bi-directional relationship. 
After investigating the ability of savoring to predict positive affect (and vice versa) and eudaimonia, the mediational role of savoring and positive affect is tested. As previously discussed, savoring is proposed to mediate the relationship between positive events and positive affect (Bryant \& Veroff, 2007). Similarly, positive affect is proposed to be a mediator that works to build resources by widening attention and cognitive repertoires (Fredrickson, 1998, 2001). Studies have supported positive affect as a mediator of particular experiences, including: the increase of resources and satisfaction after engaging in Loving Kindness Meditation (Fredrickson, et al., 2008); the duration of cardiovascular recovery from negative emotional arousal (Tugade \& Fredrickson, 2004); the level of depression and post-crisis growth resulting from individuals' trait resilience that was measured prior to the 9/11 attack on the U.S. World Trade Centre (Fredrickson, Tugade, Waugh, \& Larkin, 2003); and a recent meta-analysis discusses positive affect as a possible mediator of the finding that dispositional happiness leads to success in several life domains, from health to social to work domains (Lyubomirsky, King, et al., 2005). Fewer studies, however, have observed the mediational role of savoring on the resultant positive affect from positive life events. Therefore a combined effort was undertaken to examine both savoring and positive affect as mediators.

Along these lines, the second half of the analyses explored mediational models. First, a mediational model between everyday positive life events, savoring, positive affect and eudaimonia was considered at each time point separately. Then, a focused residualised mediation over time was conducted. The concurrent mediations at Time 1 and Time 2 compared the ability of savoring and positive affect to mediate everyday positive life events' impact on eudaimonic wellbeing. In addition the concurrent model questioned the result of positive affect from everyday positive events as mediated by savoring, and the result of eudaimonia from savoring as mediated by positive affect. In other words, one concurrent mediation model observed four constructs, which enabled the investigation of five direct relationships and five mediational relationships simultaneously. By testing these concurrent relationships at both time points, their consistency can begin to be established. Next, a more focussed observation of savoring and positive affect mediating everyday positive events, savoring, positive affect, and eudaimonia was conducted with residualised longitudinal mediational models in order to explore cause-and-effect relationships.

Lastly, to understand the influence of gender, I tested the moderating impact of gender on the positive life events, savoring, and wellbeing relationship as I navigate through the 
analyses. Since Chapter 2 found fewer gender effects than were expected, i.e., females only used one savoring strategy more than males; current hypotheses reflected these prior discoveries.

\section{Hypotheses: Adolescent savoring and wellbeing}

Four main aims guided the analyses of adolescents' savoring, positive affect and eudaimonia. These aims were: (1) to examine the consistency, stability, and interrelationships between and among the four factors of everyday savoring; (2) to investigate the direct effects of savoring on positive affect and psychological and social wellbeing, and the bi-directional relationship between savoring and positive affect, (3) to investigate the direct and indirect effects of everyday positive life events, savoring, and wellbeing concurrently and over time, and (4) examine the moderating effects of gender on the relationships between and among everyday positive life events, savoring, positive affect, and eudaimonia. From these aims, I formed three research questions and eleven related hypotheses.

(1) Are the four factors of savoring consistent across time, stable over time, and relatively independent?

Hypothesis 1: The four factors of savoring-dampening, low arousal, high arousal, and self-focused savoring-would be confirmed at 4-weeks and will be invariant between baseline and 4-weeks.

Hypothesis 2: Gender would not moderate the four factor structure of everyday savoring at either Time 1 or Time 2.

Hypothesis 3: Dampening, low arousal, high arousal, and self-focused savoring would evidence moderate to high test-retest reliability over time.

Hypothesis 4: Cross-lag relationships would be minimal over time and of less strength than stability coefficients, supporting independence of each factor of everyday savoring.

(2) Are savoring strategies predictors of positive affect and eudaimonia over time, and is the relationship between savoring and positive affect bi-directional?

Hypothesis 5: Positive savoring strategies (i.e. high arousal and self-focus) would positively predict positive affect and eudaimonia.

Hypothesis 6: Dampening savoring would negatively predict positive affect and eudaimonia.

Hypothesis 7: Positive savoring and positive affect would share a positive bi-directional relationship.

Hypothesis 8: Gender would not moderate the predicted savoring relationships. 


\section{(3) Are there mediating relationships between positive life events, savoring, positive} affect, and eudaimonia concurrently and over time?

Hypothesis 9: Savoring would mediate the relationship between everyday positive life events and positive affect, and also the relationship between everyday positive life events and eudaimonia concurrently and over time.

Hypothesis 10: Positive affect would mediate the relationship between everyday positive life events and eudaimonia, and also the relationship between savoring and eudaimonia concurrently and over time.

Hypothesis 11: Gender would not moderate either concurrent or longitudinal mediational relationships.

\section{Method}

\section{Participants and Procedure}

Participants for the present analyses were a subset of the adolescents examined in the previous analyses in Chapters 2 and 3. They were 277 adolescents who yielded data across two time points; ${ }^{22}$ however, 12 students completed less than $95 \%$ of the survey and were deleted from the sample (Tabachnick \& Fidell, 2007). ${ }^{23}$ The final sample included 265 adolescents (156 females and 1 undefined; mean age $=14$ years, range $=13$ to 15 ) obtained from four schools throughout the Bay of Plenty region of New Zealand. One school was decile 8 (contributing 35 participants), one school was decile 7 (contributing 56 participants), one school was decile 4, an all girls school (contributing 45 participants), and one school was decile 2 (contributing 129 participants). ${ }^{24}$ The sample included 66 thirteen-year-olds ( 24 male, 42 female), 141 fourteenyear-olds (53 male, 88 female) and 57 fifteen-year-olds ( 23 male, 34 female).The majority of adolescents, 70\%, were European New Zealand/Pakeha, 27\% were Māori , 4\% were Pasifika, $2 \%$ were Asian, 8\% indicated "other", and one adolescent omitted ethnicity. The sample had more European New Zealand and Māori, and less Pasifika and Asian than expected from frequencies obtained from the most recent census, which reported $68 \%, 15 \%, 7 \%$ and $9 \%$

\footnotetext{
22 At time one 410 students participated and at time two 428 students participated. A matching rate of approximately $67 \%$ represents several factors, including, missing consent forms, insufficient identifying information to match surveys, and unique students at each survey session.

${ }^{23}$ The 12 participants deleted from the matched sample were not expected to affect analyses as they comprised less than $5 \%$ of the sample (Tabachnick \& Fidell, 2001).

${ }^{24}$ School deciles are calculated using household income, occupation of parents, household crowding, educational qualifications and income support; deciles range from 1 (low socioeconomic status) to 10 (high socioeconomic status).
} 
respectively (Statistics New Zealand, 2006). See Table 4.1 for a side-by-side comparison of participants from the correlational study in Chapter 3 and this study. There was a slight increase

Table 4.1. Demographic Information Comparing the Correlational and Longitudinal Adolescent Studies.

\begin{tabular}{|c|c|c|c|c|}
\hline & \multicolumn{2}{|c|}{$\begin{array}{l}\text { Adolescent Correlational Study } \\
\qquad(N=463)\end{array}$} & \multicolumn{2}{|c|}{$\begin{array}{l}\text { Adolescent Longitudinal Study } \\
\qquad(N=265)\end{array}$} \\
\hline & $N$ & $\%$ & $N$ & $\%$ \\
\hline \multicolumn{5}{|l|}{ Gender } \\
\hline Males & 188 & 41 & 100 & 38 \\
\hline Females & 272 & 59 & 164 & 62 \\
\hline \multicolumn{5}{|l|}{ Years of age } \\
\hline 13 & 124 & 27 & 66 & 25 \\
\hline 14 & 242 & 52 & 141 & 53 \\
\hline 15 & 97 & 21 & 57 & 22 \\
\hline \multicolumn{5}{|l|}{ Ethnicity } \\
\hline European NZ & 286 & 64 & 156 & 59 \\
\hline Māori & 151 & 34 & 71 & 27 \\
\hline Pasifika & 3 & .7 & 10 & 4 \\
\hline Asian & 4 & .9 & 5 & 2 \\
\hline Other & 5 & 1 & 21 & 8 \\
\hline \multicolumn{5}{|l|}{ School Decile } \\
\hline 2 & 243 & 53 & 129 & 49 \\
\hline 4 & 72 & 16 & 45 & 17 \\
\hline 7 & 94 & 20 & 56 & 21 \\
\hline 8 & 54 & 12 & 35 & 13 \\
\hline
\end{tabular}

in females, and decrease in European New Zealanders and Māori, while Pasifika, Asian, and Other ethnicities all increased.

The procedure for Time 1 was described previously in Chapter 2. Time 2 data collection took place approximately four weeks after Time 1 with the same set of surveys in the same manner as outlined in this procedure. Signed parental consent and child assent forms were collected by the contact person before or at the time of the second data collection. For all schools, data were collected at the school, during class time. As remuneration, students were given a piece of confectionary after completing the surveys at each time point. In addition, two students were randomly drawn to win an i-Pod Nano directly after completing the second survey. And schools received two dollars for each student who completed both time points. As was described in Chapter 2, participants, parents and schools received debriefing sheets after completing the second questionnaire. In addition, schools ran a short informative piece on the 
study in the school letter. Once basic analyses were complete, schools received a short report of the findings.

\section{Measures}

The survey was composed of the same self-report measures for adolescents described in Chapters 2 and 3. The scales used for the present study measured positive life events, positive affect, savoring strategies, and eudaimonia.

Everyday Positive Life Events. The everyday Positive Life Events scale (PLE) was designed by Jose (2009) and patterned after Jose and colleagues' previous measure of Everyday Negative Life Events (Jose, et al., 1994; Jose, et al., 1998). The PLE was used to measure adolescents' rated intensity of 25 everyday positive events. Participants were asked whether an experience happened, 'yes' or 'no' (e.g. 'someone complimented you'), and 'If you said 'yes', how much of a positive experience was it?' $(0=$ none; $1=$ a little; $2=$ some; $3=$ a lot). Exploratory Factor Analysis using SPSS indicated a one-factor model, with high internal reliability at baseline $(\alpha=$ $.85)$ and 4-weeks $(\alpha=.87)$, and a moderately high test-retest reliability over the four weeks $(r=$ .56). Immediately after filling out the PLE, adolescents were asked how they responded (savored) their everyday positive events.

Positive Affect. Mood was measured using the 20-item Positive (10-items) and Negative (10-items) Affect Schedule (PANAS; Watson, Clark, \& Tellegen, 1988). ${ }^{25}$ Mood was measured using the 20-item Positive (10-items) and Negative (10-items) Affect Schedule (PANAS; Watson, Clark, \& Tellegen, 1988). Adolescents responded on a 5-point Likert scale (1 = very slightly or not at all, $2=$ a little, $3=$ moderately, $4=$ quite a bit, and $5=$ extremely) indicating how much they had experienced each mood (e.g. interested, excited, ashamed) during the past month. An average PA score was calculated for each participant. When questioned about mood occurring over the past few weeks, the PA scale has demonstrated good internal reliability and test-retest reliability over time (Watson et al.). In this sample, the PA had high internal reliability at both baseline $(\alpha=.83)$ and 4 -weeks $(\alpha=.87)$, and high test-retest reliability over the four weeks $(r=$ $.65)$.

Savoring. ${ }^{26}$ Cognitive and behavioural savoring strategies were again measured using the abridged 30-item version of the original 60-item Ways of Savoring Checklist (WOSC) (Bryant \& Veroff, 2007) described and investigated in Chapter 2. Answers were given along a 7-point Likert scale $(1=$ strongly disagree; $4=$ neutral; $7=$ strongly agree $)$ indicating how adolescents

\footnotetext{
${ }^{25}$ See Appendix K for the Positive and Negative Affect Schedule.
} 
responded to positive events during the past month. Twenty items were subjected to confirmatory factor analysis (CFA) previously reported in Chapter 2, revealing that a four-factor structure of savoring by using dampening, low arousal, high arousal, and self-focused savoring of everyday positive events fit the data well for 463 adolescents $\left(\chi^{2} / \mathrm{df}=2.55\right.$, CFI $=.93$, RMSEA $=.06, \mathrm{SRMR}=.04)$.

Eudaimonia. Adolescents responded on a 6-point Likert scale to the 12 -item version of Keyes's (2005b, 2006) Mental Health Short form that measures emotional (3-items), psychological (4-items), and social wellbeing (5-items). The measure has often been used to indicate the rates or level of mental health in a population or individual. Rates of mental health averaged over the two time points for the 265 adolescents in this study were similar to Keyes' (2006) findings using a continuous categorisation system: an averaged total between 1-2 indicates languishing mental health, 3-4 indicates moderate mental health, and 5-6 indicates flourishing. Keyes found that $6.3 \%$ of United States adolescents aged 12 to 18 were languishing, $55.4 \%$ were moderate, and 38.3\% were flourishing. Similarly, of the 265 adolescents 13 to 15 years old in this study, $7.5 \%$ were languishing, $55.8 \%$ were moderate, and $36.7 \%$ were flourishing, showing that U.S. and New Zealand adolescents have similar rates of mental health.

I did not want to confound positive affect with the emotional factor from the Mental Health Short form, therefore emotional wellbeing was omitted and psychological and social wellbeing were combined to compose a eudaimonic measure of wellbeing; for example, 'In the last month you felt: That you have warm and trusting relationships with others', and 'That people are basically good' $(1=$ never; $2=$ once or twice a week; $3=$ about once a week; $4=$ two or three times a week; $5=$ almost every day; $6=$ every day). The internal reliability of eudaimonic wellbeing was high at baseline $(\alpha=.84)$ and at 4-weeks $(\alpha=.86)$, and the test-retest reliability $(r$ $=.55)$ was moderately high.

\section{Results}

\section{Analytic Strategy}

Below I describe analyses for each of the four research questions and related hypotheses. All analyses were conducted using statistical equation modelling in AMOS (Arbuckle, 2009). In testing the hypotheses I proceeded sequentially. First, I sought to confirm the four factors of everyday savoring, and run further tests of its reliability and validity. This was done by using structural equation modelling with latent dampening, low arousal, high arousal, and self-focused savoring variables in AMOS (Arbuckle, 2009). In addition to analysing the factor structure for the current sample at both time points, I also ran two analyses of variance; one to test the degree 
to which relations among the savoring variables are stable over time, in other words, to test their stationarity (Kenny, 1979; MacKinnon, 2008), and another to observe whether the savoring structure varies depending on adolescents' gender.

Following on from these analyses, I tested the ability of the four savoring strategies to predict positive affect and wellbeing over time in a two-step approach. First, the savoring construct was combined with the positive affect and eudaimonia constructs at Time 1 and Time 2 to construct a measurement model and confirm the content validity of savoring, positive affect, and eudaimonia, before moving to the second step of testing the predicted relationships between savoring and wellbeing over time in a path model (Anderson \& Gerbing, 1988). And last, the potential moderation effect of gender on the final model was tested.

Of note, the latent path models that tested stability, cross-lags, and direction of effects were expected to violate the recommended guidelines for participant-to-parameter ratio due in part to the attrition of participants over time. In general a sample size of 200 or more will support adequate performance of statistical indices and yield meaningful and interpretable results, however, varying suggestions for participant-to-parameter ratios exist that range from 3 to 10 participants per parameter (Bollen, 1989; Quintana \& Maxwell, 1999). To stay as close as possible to the ratio guidelines, parcelling was first considered, however, stability and cross-lag analyses supported the relative independence of the four savoring strategies. Therefore latent path models were conducted with all four savoring strategies in order to retain the independent nature of the savoring factors and account for error. These latent models were then followed by observed models where participant-parameter ratios were adequate, but where the models were unable to account for exogenous error. Next, data were analysed concurrently and then longitudinally with latent path analyses in order to investigate the fourth research question: Are there mediating relationships between positive life events, savoring, positive affect, and eudaimonia concurrently and over time? First, the constructs were observed within each time point, at Time 1 and then at Time 2. To test whether the concurrent relationships were consistent across time, I then ran analyses of stationarity (Kenny, 1979; MacKinnon, 2008). Last, an analysis of invariance was used to test the moderation effect by gender on the concurrent mediational models within each time point.

Second, over time relationships were modelled using residualised latent modelling. Two sets of residualised mediational models were conducted. One set modelled the predictor and mediator at Time 1, and the outcome at Time 2. And one set modelled the predictor at Time 1, and the mediator and outcome at Time 2. An example of a model from the first set would be 
that the increase of eudaimonia at 4-weeks from positive events at baseline is mediated by baseline positive affect. An example of a model from the second mediational set would be that the increase of eudaimonia at 4-weeks from baseline positive life events is mediated by positive affect at 4-weeks. The final analysis tested the moderation effect of gender on the residualised longitudinal mediational models.

\section{Reliability and validity: Are the four factors of savoring stable over time and would concurrent relationships be replicated across time?}

As with the previous two chapters, the goodness of fit of models was assessed using four indices: $\chi^{2} / \mathrm{df}$, CFI, RMSEA, and sRMR (Hu \& Bentler, 1999). First, the ratio of the chi square value divided by the degrees of freedom was used, where a ratio of less than 3 for the $\chi^{2} / \mathrm{df}$ was considered a good fit (Kline, 2005). Second, the comparative fit index (CFI) measured incremental fit where values above .90 or .95 indicated a good fit (Bentler, 1990; Bentler \& Bonnett, 1980; Hu \& Bentler, 1999). Third and fourth, absolute fit was measured with two fit indices, root mean square error of approximation (RMSEA) (Steiger, 1990) and the standardized version of Jöreskog and Sörbom's (1982) root mean square residual (sRMR); Hu and Bentler suggested cut-off values of .06 for RMSEA and .08 for sRMR.

Confirmatory Factor Analysis at 4-Weeks. First a CFA was conducted with the 265 adolescents who participated at the 4-week follow-up to confirm the four-factor savoring model that was found with the total Time 1 group of 463 adolescents reported in Chapter 2. To test Hypothesis 1, the same items were loaded onto each of the latent variables as was described in Chapter 2. Three items were used for dampening, three items for low arousal, four items for high arousal, and three items for self-focused savoring-making a total of 13 items. The participant to parameter ratio was an acceptable 5.3. At 4-weeks, the four-factor structure of savoring yielded an acceptable fit $\left(\chi^{2} / \mathrm{df}=2.06, \mathrm{CFI}=.94\right.$, RMSEA $\left.=.06, \mathrm{sRMR}=.05\right)$ supporting the first hypothesis. All 13 items yielded significant loadings on their respective latent variable at the level of $p<.001$. The standardised regression weights for the items ranged from .35 to .82 , with an average of .64. As was found with the total Time 1 adolescent group, the correlations between latent variables were moderate to high, from .40 to .88 .

Stationarity. The degree to which relations among variables are stable over time has been termed stationarity (Kenny, 1979; MacKinnon, 2008). To test the stationarity and further test Hypothesis 1 of the four factor savoring structure, a test of invariance between study waves was conducted to discover whether factor loadings, variances, and covariances differed over a month. A comparison of the chi square value and degrees of freedom between the baseline (i.e. 
all parameters freely estimated) and the fully constrained model indicates whether or not a model violates the assumption of invariance. A significant change in the chi square value and degrees of freedom between models implies that there was variance over time in the relations among savoring factors, while a non-significant change indicates an invariant structure of savoring over time. This comparison between the baseline $\left(\chi^{2}=214.43, \mathrm{df}=116\right)$ and fully constrained models $\left(\chi^{2}=237.59, \mathrm{df}=136 ; \Delta \chi^{2}=23.16, \Delta \mathrm{df}=20, p=.28\right)$ yielded a non-significant $\mathrm{p}$-value, suggesting that the model is invariant over time; in other words, the four-factor model of savoring is sufficiently stationary. Overall, the first hypothesis was confirmed. The four factor savoring structure was found to be a valid model of everyday savoring at follow-up, four weeks after Time 1, and a reliable measure of savoring over a month.

Gender Moderation Effects. The study next tested whether gender moderated the four factor savoring model at Time 1 and Time 2. To investigate Hypothesis 2, a test for the effect of gender was conducted on the measurement models to explore whether males and females differed in their factor loadings, variances, and covariances for dampening, low arousal, high arousal, and self-focused savoring at Time 1 and at follow-up, four weeks later. At Time 1 there was no significant difference in males and females on the four savoring factors; the baseline model's chi square value and $\mathrm{df}\left(\chi^{2}=181.41, \mathrm{df}=118\right)$ and the fully constrained model's chi square value and $\mathrm{df}\left(\chi^{2}=197.99, \mathrm{df}=118\right)$ were found to be not different and hence invariant $\left(\Delta \chi^{2}=16.58, \Delta \mathrm{df}=18, p=.55\right)$. Similar non-significant results for gender were found at 4weeks. The baseline model $\left(\chi^{2}=203.49, \mathrm{df}=118\right)$ did not significantly vary from the fully constrained model $\left(\chi^{2}=223.92, \mathrm{df}=136 ; \Delta \chi^{2}=20.43, \Delta \mathrm{df}=18, p=.31\right)$, indicating that adolescent males and females yielded similar relationships among their four ways of savoring everyday positive events. That gender was found to not be a moderator of the four factor structure of savoring in adolescents validates the second hypothesis.

Test-retest Reliability and Internal Validity. The full WOSC scale has not been examined over time for test-retest reliability nor have relationships between the factors been examined, to my knowledge. We will now consider these two issues for the current abridged version of everyday savoring by testing Hypotheses 3 and 4. Although savoring strategies are processes rather than traits, they need to be at least moderately stable over time to support their reliability, Hypothesis 3. Given the moderate to high positive relationships between savoring strategies at one point in time (concurrent data) it is also important to examine the internal validity of the individual savoring styles over time, Hypothesis 4. To both observe stability and 
possible inter-relationships, a two-step approach was adopted. First longitudinal data from the abridged WOSC at baseline and 4-weeks were subjected to path analysis using AMOS (Arbuckle, 2009), where direct paths from individual baseline variables predicted only themselves at 4-weeks to test the stability of each savoring strategy. Second, the model was fully saturated (i.e. all crosslags were added) and then pruned from the least significant to the last non-significant cross-lag to determine the degree of overlap among these four processes over time.

Test-retest reliability between abridged WOSC scores at baseline and 4-weeks returned an adequate model fit $\left(\chi^{2} / \mathrm{df}=1.62, \mathrm{CFI}=.92, \mathrm{RMSEA}=.05, \mathrm{sRMR}=.06\right)$. In accordance with the third hypothesis, all four standardised stability coefficients were significant at the $p<$ .001 level with moderately high to high standardised stability coefficients: dampening $(\beta=.51)$, low arousal $(\beta=.54)$, high arousal $(\beta=.67)$, and self-focused savoring $(\beta=.70) .{ }^{27} \mathrm{Next}$, the initial exploratory base model allowed each latent variable at baseline to predict each latent variable at four weeks making a total of 16 structural paths. Although the data fit for the saturated model was adequate $\left(\chi^{2} / \mathrm{df}=1.64, \mathrm{CFI}=.92, \mathrm{RMSEA}=.05, \mathrm{sRMR}=.05\right)$, no significant cross-lags were obtained in this model. Therefore non-significant cross-lags were individually pruned in order (up to $p<.05$ ). At each stage of the pruning process the beta weights, phis, and error terms were assessed to ensure multicollinearity was not a significant problem. The pruning took 11 steps and yielded one significant cross-lag from high arousal savoring at Time 1 to self-focused savoring at Time $2(\beta=-.53, p<.05)$ and all stability paths remained highly significant $(p<.001)$, supporting the fourth hypothesis that cross-lag relationships over time would be minimal. ${ }^{28}$

The four-factor model of everyday savoring of everyday positive events appears moderately stable over four weeks. It is noteworthy that given the moderate to high correlations between latent variables at a given time point (i.e. covariances in the CFAs reported in Chapter 2), that only one cross-lag over time was significant. The one significant cross-lag, from high arousal to self-focus, indicates that although at any given time high arousal and self-focused

\footnotetext{
${ }_{27}$ The participant to parameter ratio, however, was 2.19, which was below the lower recommended threshold of 3 . Therefore, an observed stability model was also conducted, returning an acceptable participant-parameter ratio of 9.46, and model fit: $\chi^{2} / \mathrm{df}=2.34, \mathrm{CFI}=.98, \mathrm{RMSEA}=.07, \mathrm{sRMR}=.06$. The standardised stability coefficients were all highly significant $(p<.001)$, but weaker $($ dampening $=.33)$, (low arousal $=.30)$, (high arousal $=.50)$, and (self-focus $=.43)$. The weaker stabilities may be attributable to less correlated error in the observed model. 28 An observed cross-lag model was conducted that stipulated the same paths as the final pruned test-retest latent path model where high arousal predicted self-focused savoring, however, this cross-lag relationship was nonsignificant. Therefore the final test-retest observed model returned the same model found with the analysis of stability (see footnote 27).
} 
savoring are highly and positively related (see Figure 2.1 in Chapter 2), over time high arousal savoring may interfere with and inhibit self-focused savoring for adolescents. Arguably, the lack of cross-lag relationships suggest that the four savoring processes for everyday positive life events generally occur in isolation from each other for adolescents over one month.

Are savoring strategies predictors of positive affect and eudaimonia over time, and is the savoring and positive affect relationship bi-directional?

Measurement Models. To test Hypotheses 5, 6, and 7, measurement models were formed and tested before testing path models. First positive affect and eudaimonia were parcelled, which was then combined with savoring to create a measurement model of everyday savoring, positive affect, and eudaimonia. This measurement model was tested at baseline and at 4-weeks, along with the possible gender moderation effects before testing the hypothesised path models.

The four factors of savoring were combined with a parcelled measurement model of positive affect and eudaimonia. (The parcelled eudaimonia model was the same as the one derived in Chapter 3.) The positive affect model had three parcels where items were randomly assigned: parcels 1 and 2 had 3 items each, and parcel 3 had four items. The participant to parameter ratio was acceptable at 3.35. Both measurement models yielded a good fit to the data, although the baseline model $\left(\chi^{2} / \mathrm{df}=1.32, \mathrm{CFI}=.97, \mathrm{RMSEA}=.04, \mathrm{SRMR}=.05\right)$ had slightly better model fit than the model at 4-weeks $\left(\chi^{2} / \mathrm{df}=1.69, \mathrm{CFI}=.95, \mathrm{RMSEA}=.05\right.$, SRMR $=$ .05). All factor loadings at baseline were significant at the level of $p<.001$, except for savoring item 19, which, recall, was double-loaded on low arousal $(p<.05)$, and dampening $(p<.01)$. All factor loadings at 4 -weeks were significant at the level of $p<.001$. The overall mean of the standardised regression coefficients for factor loadings at baseline was .66, and all factor loadings fell within .23 and .85 ; the overall mean of the standardised regression coefficients for factor loadings at 4-weeks was .66, and all factor loadings fell within .37 and .90. The overall mean squared multiple correlation was similar between the baseline (.50) and 4-week (.48) models, indicating acceptable criterion validity for both models. The correlations between concurrent latent variables at baseline and 4-weeks are reported in Table 4.2. 
Table 4.2. Concurrent Correlations among Latent V ariables for Baseline and 4-week. Measurement Models of Savoring, Positive Affect, and Eudaimonia.

\begin{tabular}{lcccccc}
\hline & Dampen & LA & HA & SF & PA & Eudaimonia \\
\hline Dampening & - & $.67^{* * *}$ & $.34^{* *}$ & $.52^{* * *}$ & $.32^{*}$ & $.23^{*}$ \\
Low Arousal & $.41^{* * *}$ & - & $.63^{* * *}$ & $.75^{* * *}$ & $.41^{* * *}$ & $.37^{* * *}$ \\
High Arousal & $.33^{* * *}$ & $.69^{* * *}$ & - & $.75^{* * *}$ & $.47^{* * *}$ & $.35^{* * *}$ \\
Self-focus & $.62^{* * *}$ & $.88^{* * *}$ & $.86^{* * *}$ & - & $.69^{* * *}$ & $.63^{* * *}$ \\
Positive Affect & $.15^{*}$ & $.22^{*}$ & $.35^{* * *}$ & $.51^{* * *}$ & - & $.55^{* * *}$ \\
Euadaimonia & $.13^{\dagger}$ & $.32^{* * *}$ & $.32^{* * *}$ & $.47^{* * *}$ & $.52^{* * *}$ & - \\
\hline
\end{tabular}

Note $\mathrm{LA}=$ Low Arousal, $\mathrm{HA}=$ High Arousal, $\mathrm{SF}=$ Self-focus, and $\mathrm{PA}=$ Positive Affect. Values above the diagonal represent baseline correlations; values below the diagonal represent 4 -week correlations. ${ }^{* * *} p<.001,{ }^{* *} p<.01,{ }^{*} p<.05$, $t p<.10$.

Although the correlations, at baseline and 4-weeks, between the four savoring factors were generally moderate to high $(M=.51)$, Chapter 3 supported their discriminant validity, and the test of cross-lags in this study indicated one significant and negative relationship from high arousal to self-focused savoring. The similarity and good fit of the baseline and 4-week measurement models indicate adequate content validity of everyday savoring, positive affect, and eudaimonia.

Gender Moderation Effects. A test for the effect of gender was conducted on the measurement models of savoring, positive affect, and eudaimonia to explore whether males and females differ in their factor loadings, variances, and covariances at Time 1 and at Time 2. At Time 1 there was no significant difference in models; the baseline model's chi square and $\mathrm{df}\left(\chi^{2}\right.$ $=343.22, \mathrm{df}=272)$ and the fully constrained model's chi square and $\mathrm{df}\left(\chi^{2}=377.04 \mathrm{df}=307\right)$ were invariant $\left(\Delta \chi^{2}=33.82, \Delta \mathrm{df}=35, p=.52\right)$. Similar non-significant results for gender were found at 4-weeks. The baseline model $\left(\chi^{2}=394.55, \mathrm{df}=272\right)$ did not significantly vary from the fully constrained model $\left(\chi^{2}=431.50, \mathrm{df}=272 ; \Delta \chi^{2}=36.98, \Delta \mathrm{df}=35, p=.38\right)$, meaning that adolescent males and females similarly engaged in everyday savoring, positive affect, and eudaimonic wellbeing.

Structural models over time. After the measurement models were found to fit the data well, structural path models were conducted to test for predicted directions of effect over time. 
Causal paths were stipulated and model refinement was made by pruning paths from least to most significant, stopping at the value of $p<.05$ (Byrne, 2001; Kline, 2005).

First, a residualised longitudinal path model was conducted with the 6 latent variables: dampening, low arousal, high arousal, and self-focused savoring, and positive affect, and eudaimonia, at the two times of measurement. All latent variables were allowed to correlate concurrently with each other at baseline and at 4-weeks based on their moderate to high significant correlations evident during analyses of measurement models. The error term for each indicator was allowed to correlate with itself over time from baseline to 4-weeks, for instance, error for indicator 1 on dampening at baseline was allowed to correlate with error for indicator 1 on dampening at 4-weeks. Each latent variable at baseline was allowed to predict itself at 4-weeks (i.e. four stability paths). To test Hypotheses 5 and 6, each of the four savoring factors was allowed to predict positive affect and eudaimonia, and positive affect was allowed to predict eudaimonia. Specific paths were stipulated at this stage, rather than using a saturated model for 2 reasons: (1) the paths from savoring to positive affect and eudaimonia, and the path from positive affect to eudaimonia were an extension of the predictions made with the concurrent data in Chapter 3, and (2) a predicted model with limited paths would put less strain on the participant to parameter ratio than a saturated model.

The base model yielded an adequate fit to the data $\left(\chi^{2} / \mathrm{df}=1.42, \mathrm{CFI}=.93\right.$, RMSEA $=$ .04$, sRMR $=.05)$ with all four stability paths significant at $p<.001$, and one marginal cross-lag from self-focused savoring to positive affect $(\beta=.57, p=.07)$. Each non-significant path was individually removed, sequentially from least to most significant. At each stage of pruning, the model fluctuations in beta weights, phis, and error terms were assessed to ensure multicollinearity was not a significant problem. The pruned model improved the $\chi^{2} / \mathrm{df}$ ratio index to 1.41 and the CFI to .94, while the other fit indices remained identical to the base model. After pruning 5 non-significant paths, a new path from baseline dampening savoring to positive affect $(\beta=-.19, p<.05)$, and a new path from positive affect to eudaimonia at 4-weeks were revealed $(\beta=.21, p<.01)$. The strength decreased for the path from self-focused savoring to positive affect $(\beta=.34, p<.01)$. Hypothesis 5 was partially confirmed by the finding that selffocused savoring positively predicted positive affect; however, unexpectedly, high arousal savoring did not predict positive affect. Hypothesis 6 was confirmed by the finding that dampening savoring negatively predicted positive affect. Although high arousal negatively predicted self-focused savoring during the cross-lag analysis it did not appear to predict self- 
focused savoring in a more complex model. See Figure 4.1 for the pruned residualised latent path model of the hypothesised relationships between savoring, positive affect, and eudaimonic wellbeing.

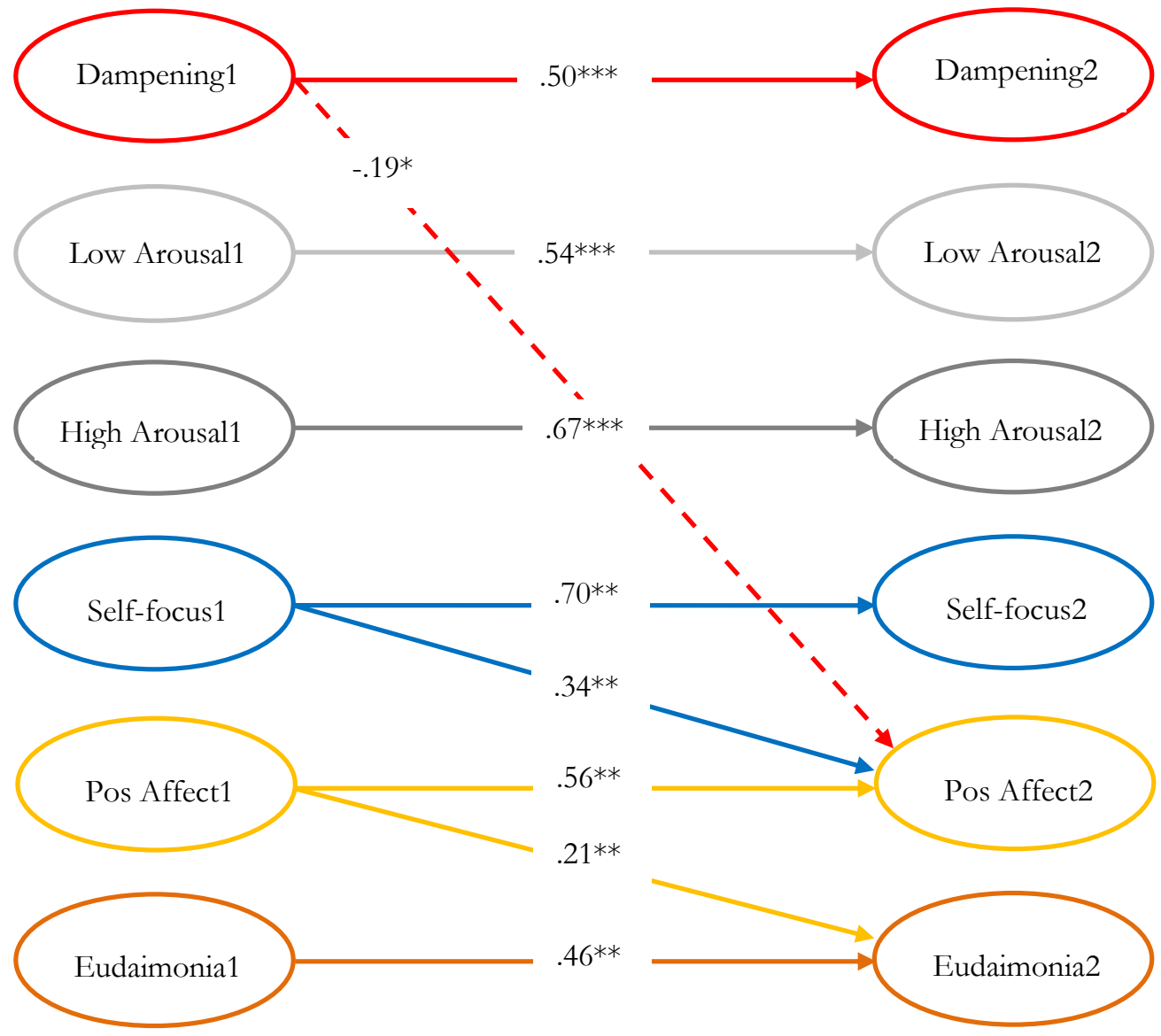

Figure 4.1. Pruned model of savoring, positive affect, and eudaimonia over four weeks. Note. Solid lines indicate a positive relationship and dashed lines indicate a negative relationship.

An observed variable model was also conducted since the residualised path model had a 1.35 participant to parameter ratio, which is considerably below the threshold of 3 . The observed model was stipulated to have the same paths as the pruned residualised latent path model and had a participant to parameter ratio of 4.73 . The model yielded an adequate model fit $\left(\chi^{2} / \mathrm{df}=\right.$ $2.21, \mathrm{CFI}=.97, \mathrm{RMSEA}=.07, \mathrm{sRMR}=.06$ ), however, the path from dampening to positive affect was found to be non-significant $(p=.33)$.

Gender moderation effects. The impact of gender on the cross-lag relationships from dampening savoring to positive affect, self-focused savoring to positive affect, and positive affect to eudaimonia was tested for the residualised latent path model in accordance with Hypothesis 8. 
The chi square value and degrees of freedom for the fully constrained baseline model $\left(\chi^{2}=\right.$ $556.04, \mathrm{df}=452)$ did not significantly vary from the constrained cross-lag model $\left(\chi^{2}=558.32\right.$, df $\left.=455 ; \Delta \chi^{2}=2.28, \Delta \mathrm{df}=3, p=.52\right)$. In support of Hypothesis 8 , these findings suggest adolescent males and females share similar causal paths between everyday positive events, savoring, and wellbeing over one month, as depicted in Figure 4.1.

Second, to test Hypothesis 7 a focused model was conducted to investigate the evidence that self-focused savoring and positive affect share a reciprocal relationship. The fully saturated model returned a good model fit $\left(\chi^{2} / \mathrm{df}=1.18, \mathrm{CFI}=.99, \mathrm{RMSEA}=.04, \mathrm{sRMR}=.04, p=.20\right)$ with strong and significant stability coeffecients $(\phi<.001)$ and one significant cross-lag from self-focused savoring to positive affect $(\beta=.23, p<.05)$, however, the cross-lag from positive affect to self-focused savoring was clearly non-significant $(\beta=-.09, p=.53)$. After pruning the non-significant cross-lag, the model $\chi^{2} / \mathrm{df}$ ratio and $\mathrm{p}$-value improved slightly $\left(\chi^{2} / \mathrm{df}=1.16, p=\right.$ .22), the CFI, RMSEA and sRMR values remained the same, and the strength of the cross-lag from self-focused savoring to positive affect decreased slightly $(\beta=.21, p<.05)$. In this case Hypothesis 7 was not supported, and it appears that the relationship between engaged savoring and positive affect, at least over four weeks, was unidirectional-from savoring to positive affect, but not the reverse (Figure 4.2).
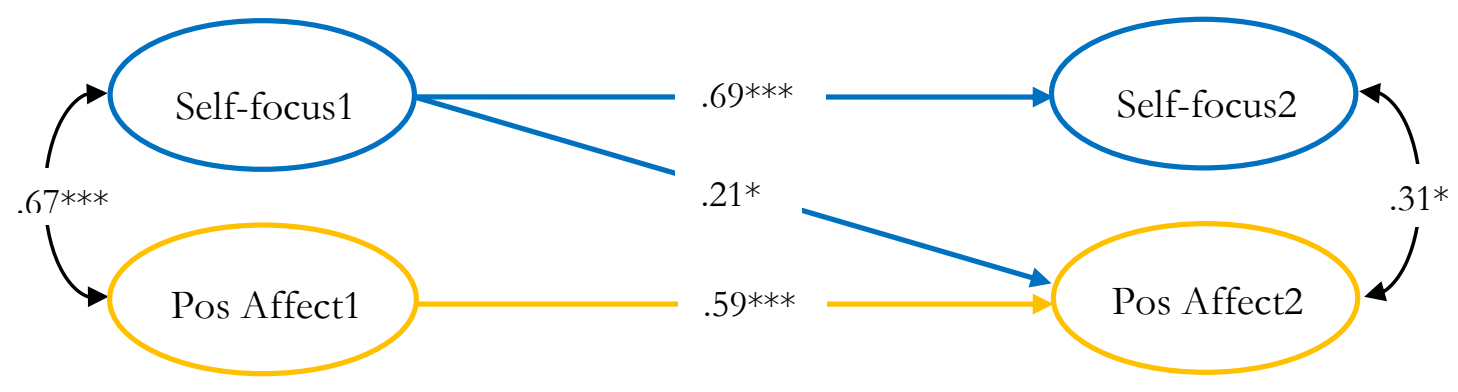

Figure 4.2. The standardised pruned model for self-focus savoring and positive affect over four weeks.

\section{Structural mediational models: Are there mediating relationships between positive life events, savoring, positive affect, and eudaimonia concurrently and over time?}

The longitudinal latent variable path model suggested a mediational relationship where savoring might indirectly impact eudaimonia through positive affect, a possibility that could be tested by conducting focussed structural mediational models. The mediational relationship between positive affect, eudaimonia, and savoring was tested while including positive life events. 
The focussed models decreased the strain on parameters and enabled the inclusion of positive life events, which in turn allowed the testing of Hypotheses 9 and 10.

Mediational models were first tested separately at baseline and at 4-weeks to investigate concurrent relationships, which were then subjected to invariance testing to discover whether the mediational relationships were consistent across time. Second, baseline and 4-week data were combined to test direct and indirect relationships between and among positive life events, savoring, positive affect, and eudaimonia over time. Finally, each of the focused mediational models was subjected to tests of moderation by gender. The saturated mediational model tested concurrently and longitudinally is presented in Figure 4.3.

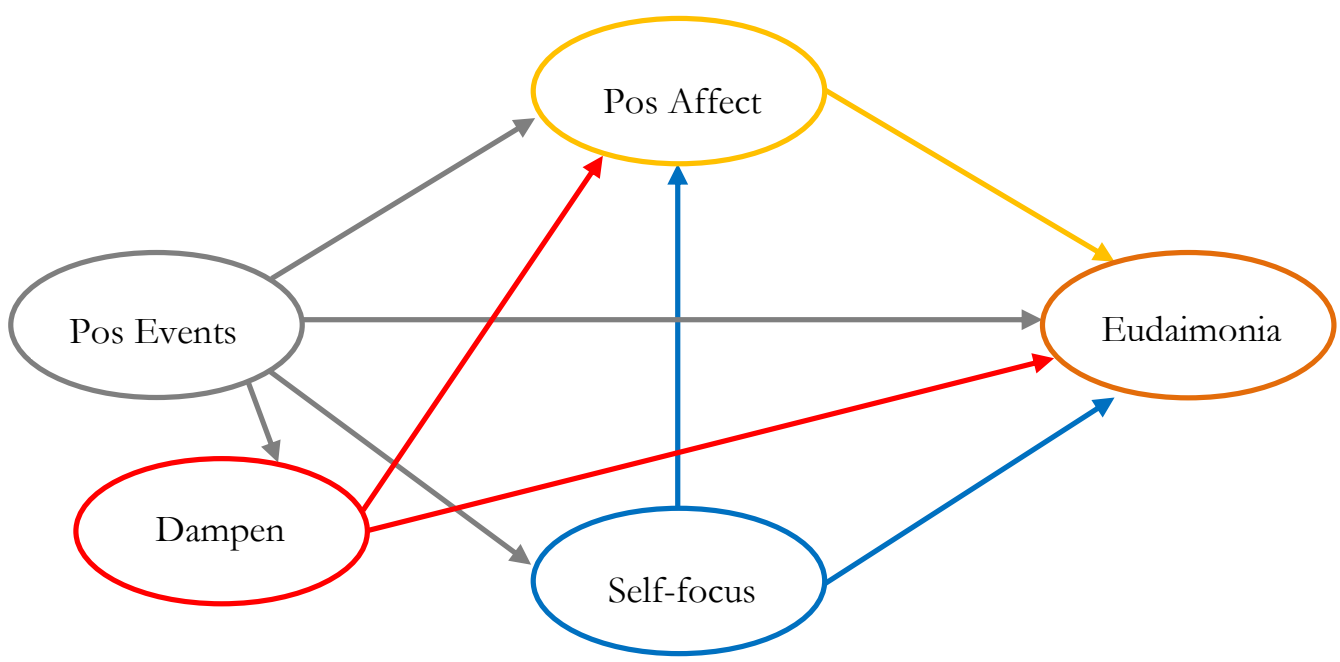

Figure 4.3. Fully saturated mediational model of everyday positive events, dampening and selffocus savoring, positive affect, and eudaimonia.

The intensity of positive life events, dampening, self-focused savoring, positive affect, and eudaimonia were included as latent variables in the concurrent mediational models (Figure 4.3). Dampening savoring was then removed from the longitudinal model because the relationship between dampening savoring and positive affect was non-significant in the concurrent mediational model (discussed below). Four mediational relationships were stipulated by Hypotheses 9 and 10: (1) savoring would mediate the relationship from positive life events to positive affect, (2) savoring would mediate the relationship from positive life events to eudaimonia, (3) positive affect would mediate the relationship from savoring to eudaimonia, and (4) a prediction supported by the results of the residualised longitudinal path model was that positive affect would mediate the relationship from savoring to eudaimonia. The four 
mediational models were simultaneously tested by using structural equation modelling. Assessing the four mediational paths simultaneously allowed the relative strengths of each pathway to be compared.

Concurrent Mediation. The initial Time 1 model was fully saturated (Figure 4.3). Everyday positive life events was the only exogenous variable and it was allowed to directly predict dampening and self-focused savoring, positive affect, and eudaimonia. Dampening and self-focused savoring was allowed to directly predict positive affect and eudaimonia, and positive affect was allowed to directly predict eudaimonia. The model fit the data well, $\chi^{2} / \mathrm{df}=1.38, \mathrm{CFI}$ $=.98, \mathrm{RMSEA}=.04, \mathrm{sRMR}=.06$. Positive life events significantly and positively predicted positive affect, dampening savoring, and self-focus savoring. In addition, positive affect positively predicted eudaimonia, and self-focused savoring positively predicted both positive affect and eudaimonia. However, several paths were non-significant and therefore removed in sequence from most non-significant to least non-significant up to $p<.05$, which included: positive life events to eudaimonia, dampening to positive affect, and dampening to eudaimonia. Since dampening savoring did not significantly predict either wellbeing type, it was also removed. The final pruned model resulted in an improved model fit, $\chi^{2} / \mathrm{df}=1.24, \mathrm{CFI}=.99$, RMSEA $=$ $.03, \mathrm{sRMR}=.04$, seen in Figure 4.4. As expected, everyday positive life events directly predicted

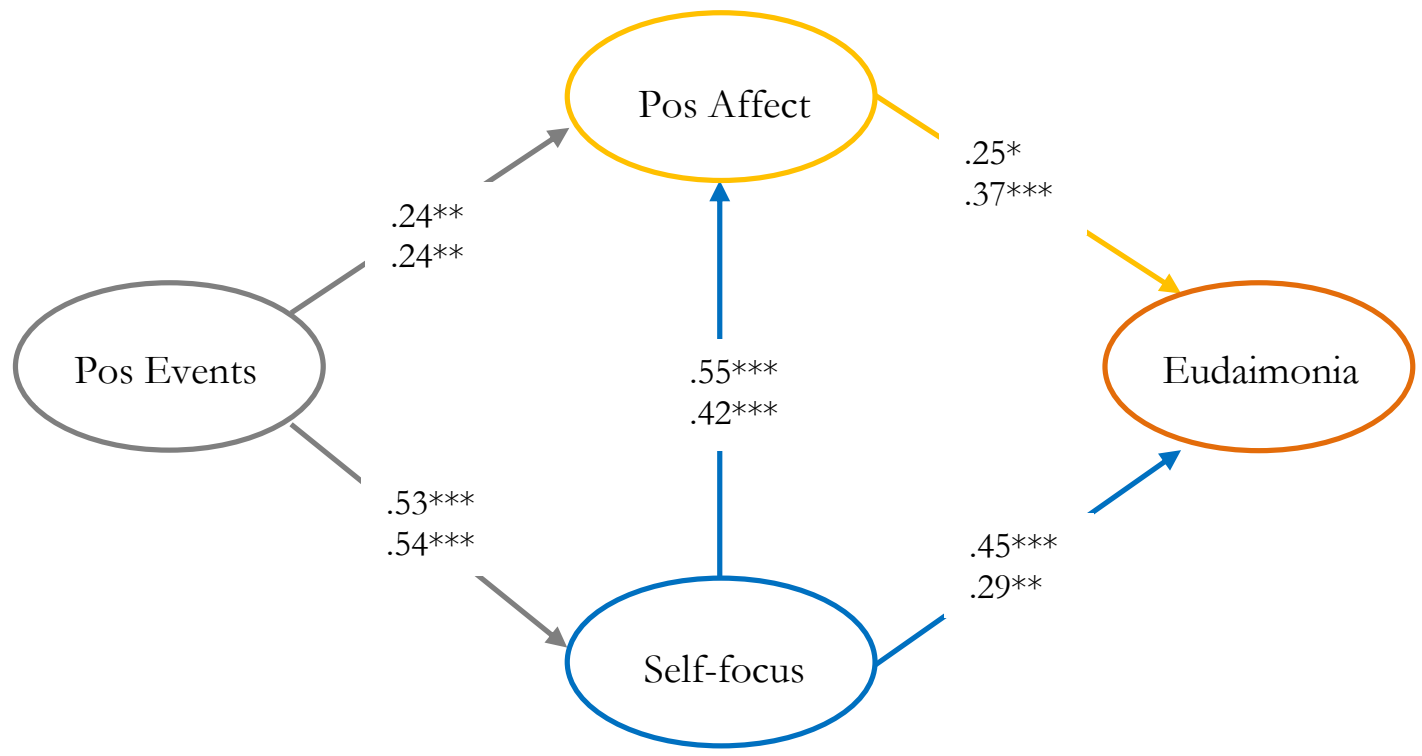

Figure 4.4. Pruned concurrent mediational path models of positive life events, savoring, positive affect, and eudaimonia. Note. the standardised direct effects at baseline are the top values; the standardised direct effects at 4-weeks are the bottom values. ${ }^{* *} p<.001,{ }^{* *} p<.01,{ }^{*} p<.05$. 
self-focused savoring $(\beta=.53, p<.001)$ and positive affect $(\beta=.24, p<.01)$. Also as expected self-focus savoring directly predicted positive affect $(\beta=.55, p<.001)$ and eudaimonia $(\beta=.45$, $p<.01)$, however, it was unexpected that dampening savoring was unable to predict either types of wellbeing.

Indirect relationships were investigated using the bootstrap method with maximum likelihood estimation in AMOS (Arbuckle, 2009; Byrne, 2001). The number of bootstrap samples was set to 200 and a bias corrected interval of 95\% was used to interpret indirect effects and confidence intervals (CI). The simultaneous indirect relationships are presented in Figure 4.5. As predicted by the ninth hypothesis, the indirect effect of positive events on positive affect,

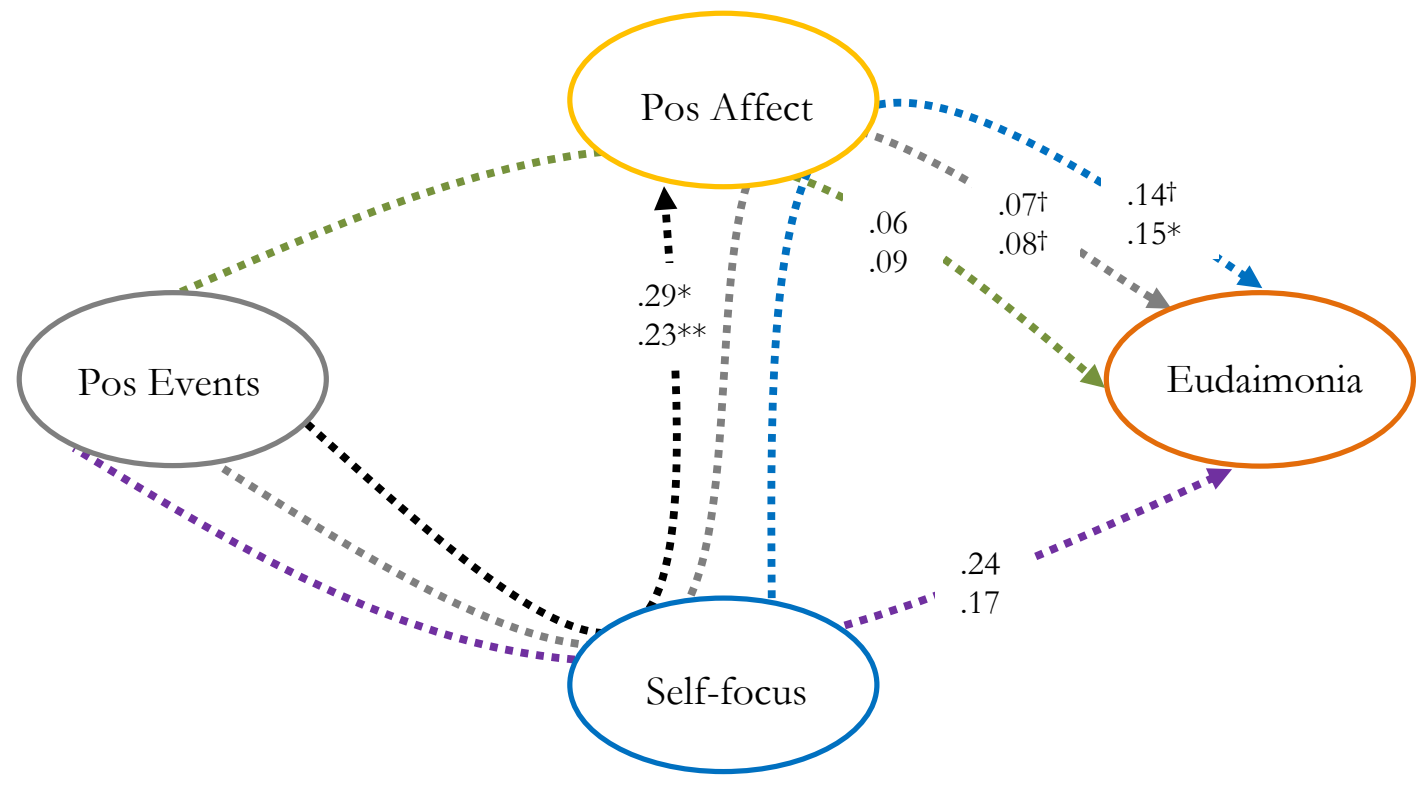

Figure 4.5. Indirect effects for the pruned concurrent mediational model of positive life events, self-focus savoring, positive affect, and eudaimonia. Note. the standardised indirect effects at Baseline are the top values; the standardised indirect effects at 4-weeks are the bottom values. ${ }^{*} p<<.01,{ }^{*} p<.05,{ }^{*} p<.10$.

through self-focus savoring, was positive and significant $(\beta=.29$, se $=.09, \mathrm{CI}=.16-.42, p<$ $.01)$. In addition, both Hypotheses 9 and 10 were supported by the findings that positive events indirectly predicted eudaimonia through positive affect (.06; a weak effect), and through savoring (.24; a weak to moderate effect), and through savoring and positive affect (.07; a weak effect). The combined indirect effects for both the positive affect and self-focused savoring mediators 
on the relationship from positive events to eudaimonia was moderate $(\beta=.37$, se $=.06, \mathrm{CI}=.27$ $-.47, p<.05)$. Lastly, and congruent with the residualised longitudinal path model and the tenth hypothesis, positive affect was found to be a positive mediator of the relationship from savoring to eudaimonia, however, the effect was marginally significant $(\beta=.14$, se $=.07, \mathrm{CI}=.03-.25, p$ $=.06)$. Figure 4.5 is a model of the simultaneous indirect relationships.

The same saturated model as the initial Time 1 model was specified at 4-weeks (Figure 4.3). Again the same paths were non-significant and therefore removed. This pruned model fit the data well, $\chi^{2} / \mathrm{df}=1.96, \mathrm{CFI}=.96, \mathrm{RMSEA}=.06, \mathrm{sRMR}=.05$, but not as well as at Time 1 (Figure 4.4). All the direct effects found at baseline were represented at 4-weeks. All the indirect relationships found at baseline were also confirmed at 4-weeks, again supporting the ninth hypothesis that savoring mediates the relationship between everyday positive events and positive affect $(\beta=.23$, se $=.08, \mathrm{CI}=.12-.39, p<.01)$, and eudaimonia $(\beta=.17)$. In support of the tenth hypothesis, the model confirmed the relationship suggested by the residualised longitudinal path model that positive affect would mediate the relationship between savoring and eudaimonia $(\beta=.15$, se $=.05, \mathrm{CI}=.09-.26, p<.01)$. The strength of each of the four indirect relationships was in the same order as at baseline (Figure 4.5).

From strongest to weakest, the strongest standardised indirect effect was everyday positive life events predicting positive affect through savoring (.23). Second, savoring mediated the relationship between everyday positive life events and eudaimonia (.17). A close third was the mediation of savoring to eudaimonia by positive affect (.15). Next, positive affect mediated the relationship between everyday positive life events and eudaimonia (.09), and last both selffocused savoring and positive affect mediated the relationship between everyday positive life events and eudaimonia (.08). Although specific significance values were unavailable for the mediational effect of positive affect and self-focused savoring on the relationship from everyday positive life events and eudaimonia, their combined effect was moderate and significant $(\beta=.33$, se $=.05, \mathrm{CI}=.24-.39, p<.05)$.

Stationarity. From the separate analyses of data at baseline and at 4-weeks it appeared that the concurrent direct and indirect relationships between positive life events, savoring, and eudaimonia were similar over the two data waves. The assumption of stationarity was subjected to invariance testing by specifying the date of data collection as the moderating variable; responses from Time 1 were the first group, and responses from Time 2 were the second group. The chi square value and degrees of freedom for the freely estimated model $\left(\chi^{2}=156.88, \mathrm{df}=\right.$ 
98) did not significantly vary from the model where the five direct relations among latent variables were constrained $\left(\chi^{2}=166.39, \mathrm{df}=104 ; \Delta \chi^{2}=9.51, \Delta \mathrm{df}=6, p=.15\right)$. These findings indicate that the relationships between adolescents' positive events, self-focused savoring, positive affect, and social and psychological wellbeing depicted in Figure 4.4 and 4.5 are a reliable representation of how adolescents experienced these phenomena concurrently, supporting Hypotheses 9 and 10.

Gender moderation effects. To test Hypothesis 11, the moderating effect of gender was explored on the final pruned concurrent model (i.e. a case of moderated mediation analysis) in Figure 4.4. First, gender was entered as the moderating variable with no path constraints for the Time 1 data $\left(\chi^{2}=115.60, \mathrm{df}=98\right)$. Then, all paths between latent variables were constrained equal, to test for invariance $\left(\chi^{2}=116.36, \mathrm{df}=103\right)$. The change in the chi square value and degrees of freedom from baseline to the fully constrained model was non-significant $\left(\Delta \chi^{2}=.76\right.$, $\Delta \mathrm{df}=5, p=.98)$, in other words, the interrelationships found between everyday positive life events, everyday self-focused savoring, positive affect, and eudaimonia were invariant between adolescent males and females. These findings were confirmed at 4-weeks, where the baseline chi square value and degrees of freedom $\left(\chi^{2}=149.21, \mathrm{df}=98\right)$ did not significantly vary from the fully constrained model $\left(\chi^{2}=157.57, \mathrm{df}=103 ; \Delta \chi^{2}=8.36, \Delta \mathrm{df}=5, p<.14\right)$. In line with Hypothesis 11, the results indicate that males and females experienced similar increases in their savoring and wellbeing from their everyday positive events, as well as similar increases in their positive affect from savoring, and increases in eudaimonia from savoring and positive affect (Figure 4.4).

Longitudinal Mediation. A total of eight focussed single mediator models were conducted to observe whether direct and indirect relationships were robust over time in accordance with Hypotheses 9 and 10. Four residualised longitudinal mediational models stipulated that both the predictor and mediator variables were from Time 1, while the outcome variable was from Time 2 (Figure 4.6). The remaining four mediational models stipulated that the predictor variable was from Time 1, while the mediator and outcome variables were from Time 2 (Figure 4.7). Each model had an acceptable parameter to participant ratio, with the lowest ratio at 3.90 parameters per participant.

The arrangement of variables was more focussed than the previous concurrent model and were as follows: (1) positive life events and eudaimonia mediated by positive affect, (2) positive life events and eudaimonia mediated by self-focused savoring, (3) positive life events and 
positive affect mediated by self-focused savoring, and (4) self-focused savoring and eudaimonia mediated by positive affect.

The saturated and pruned model fit indices for the models using mediators at Time 1 are reported in Table 4.3. For each mediational model, the variable from Time 2 was residualised (i.e.

Table 4.3. Summary of Model Fit for Saturated and Pruned Mediational Models with Mediators at Time 1.

\begin{tabular}{ccccc}
\hline \multicolumn{5}{c}{ Time 1 Mediator Models } \\
\hline Model & $\chi^{2} / \mathrm{df}$ & CFI & RMSEA & sRMR \\
\hline PLE - PA - EUD & & & & \\
Saturated & 2.18 & .96 & .07 & .09 \\
Pruned & 2.16 & .96 & .07 & .09 \\
PLE - SF - EUD & & & & \\
Saturated & 2.03 & .96 & .06 & .08 \\
Pruned & 2.00 & .96 & .06 & .08 \\
PLE - SF - PA & & & & \\
Saturated & 1.79 & .97 & .06 & .07 \\
Pruned & 1.81 & .97 & .06 & .07 \\
SF - PA - EUD & & & & \\
Saturated & 1.03 & 1.00 & .01 & .04 \\
Pruned & 1.01 & 1.00 & .01 & .04 \\
\hline
\end{tabular}

Note. The models $=$ Predictor - Mediator - Outcome. PLE $=$ positive life events, $\mathrm{PA}=$ positive affect, $\mathrm{EUD}=$ eudaimonia, $\mathrm{SF}=$ self-focused savoring.

predicted by itself at Time 1), and the error between each of the indicators was correlated. For example, the error from parcel 1 for eudaimonia at Time 1 was correlated with the error from parcel 1 for eudaimonia at Time 2 . In addition, all residualised variables were correlated with the predictor variable at Time 1, for instance, the predictor positive life events at Time 1 was correlated with eudaimonia at Time 1 (Model A, Figure 4.6), and with both positive affect at Time 1 and eudaimonia at Time 1 (Model A, Figure 4.6). Each individual model was saturated. To arrive at the final models, as seen in Figure 4.6, any non-significant paths were removed in order-from least to most significant-up to $p<.05$. 

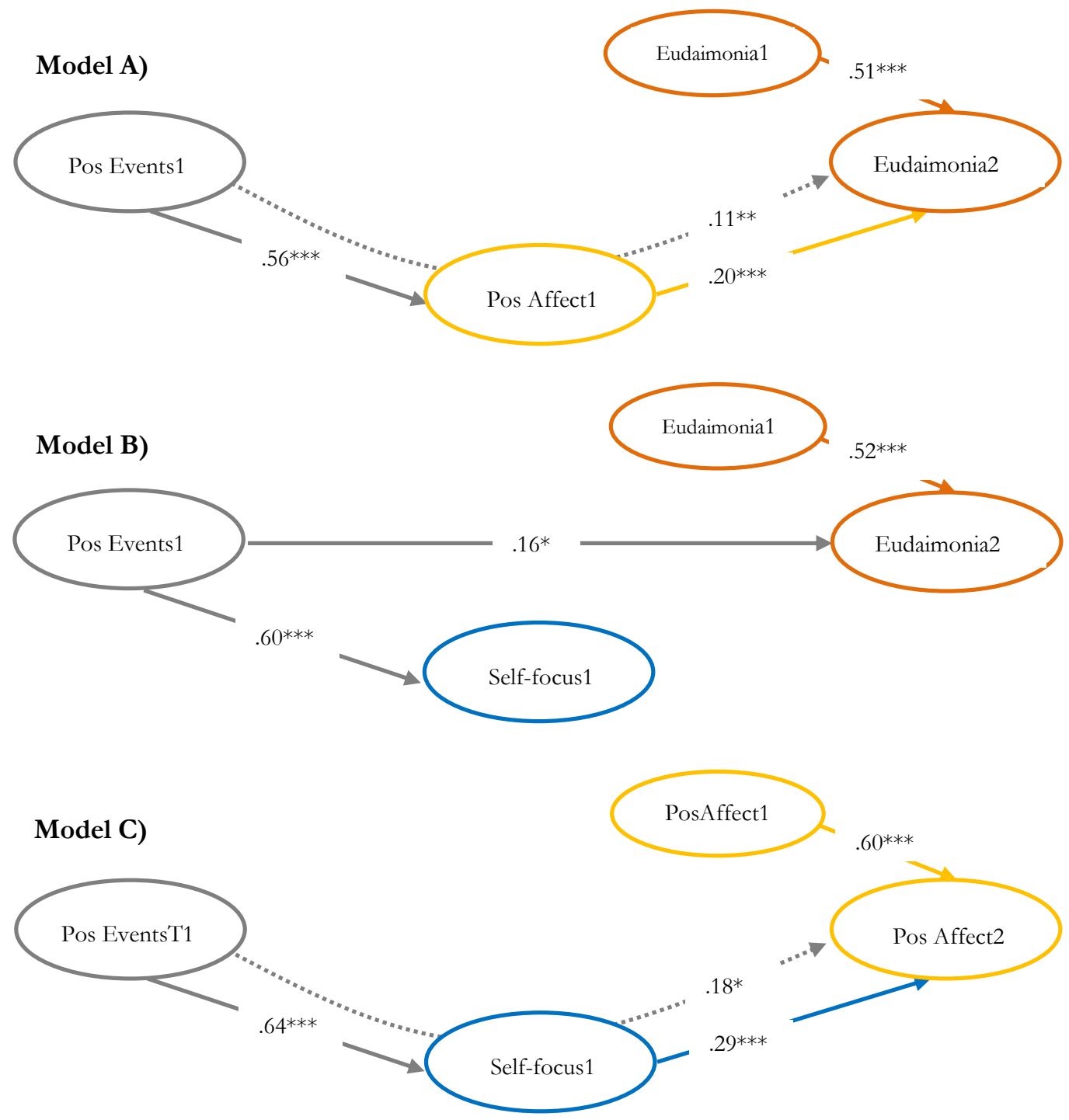

Model D)

Eudaimonia1

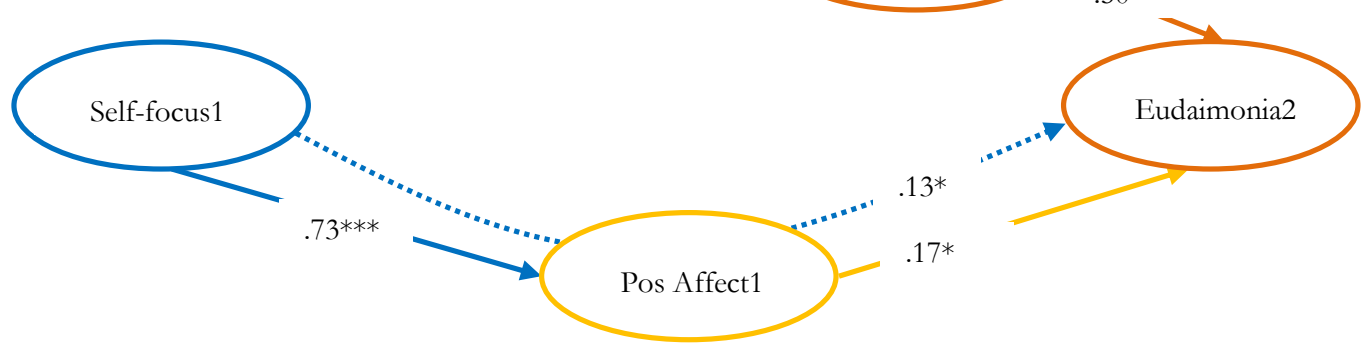

Figure 4.6. Summary of the pruned and standardised longitudinal residualised mediational path models with mediators at Time 1 . Note. The solid arrows are direct relationships, and the dashed arrows are indirect relationships 
Identical to the concurrent mediational models, indirect relationships were investigated using the bootstrap method with maximum likelihood estimation in AMOS (Arbuckle, 2009; Byrne, 2001). The number of bootstrap samples was set to 200 and a bias corrected interval of 95\% was used to interpret indirect effects and confidence intervals (CI). As predicted by Hypothesis 10, baseline positive life events and eudaimonia at 4-weeks were mediated by positive affect at baseline, a weak indirect effect $(\beta=.11$, se $=.05$, CI $=.05-.20, p<.01)$ (Model A, Figure 4.6). Contrary to Hypothesis 9, baseline positive life events and eudaimonia at 4-weeks were not mediated by self-focused savoring (Model B, Figure 4.6). In accordance, however, with Hypothesis 9, baseline positive life events and positive affect at 4-weeks were mediated by selffocused savoring at baseline, a weak indirect effect $(\beta=.18$, se $=.07, \mathrm{CI}=.06-.31, p<.05)$ (Model C, Figure 4.6), and baseline positive affect mediated baseline self-focused savoring and eudaimonia at 4-weeks, which was also a weak indirect relationship $(\beta=.13$, se $=.06, \mathrm{CI}=.04$ $-.24, p<.05)$ (Model D, Figure 4.6).

The saturated and pruned model fit indices for the models with mediators at Time 2 are reported in Table 4.4, and each final pruned model is represented in Figure 4.7. As predicted by

Table 4.4. Summary of Model Fit for Saturated and Pruned Mediational Models with Mediators at Time 2.

Time 2 Mediator Models

\begin{tabular}{ccccc}
\hline Model & $\chi^{2} / \mathrm{df}$ & CFI & RMSEA & sRMR \\
\hline PLE - PA - EUD & & & & \\
Saturated & 1.71 & .97 & .05 & .10 \\
Pruned & 1.70 & .97 & .05 & .11 \\
PLE - SF - EUD & & & & \\
Saturated & 1.23 & .99 & .03 & .04 \\
Pruned & 1.22 & .99 & .03 & .04 \\
PLE - SF - PA & & & & .05 \\
Saturated & 1.24 & .99 & .03 & .05 \\
Pruned & 1.23 & .99 & .03 & .05 \\
SF - PA - EUD & & & & .05 \\
Saturated & 1.45 & .98 & .04 & .04 \\
Pruned & 1.43 & .98 & .04 & \\
\hline
\end{tabular}

Note. The model $=$ Predictor - Mediator - Outcome. PLE $=$ positive life events, $\mathrm{PA}=$ positive affect, EUD = eudaimonia, $\mathrm{SF}=$ self-focused savoring. 


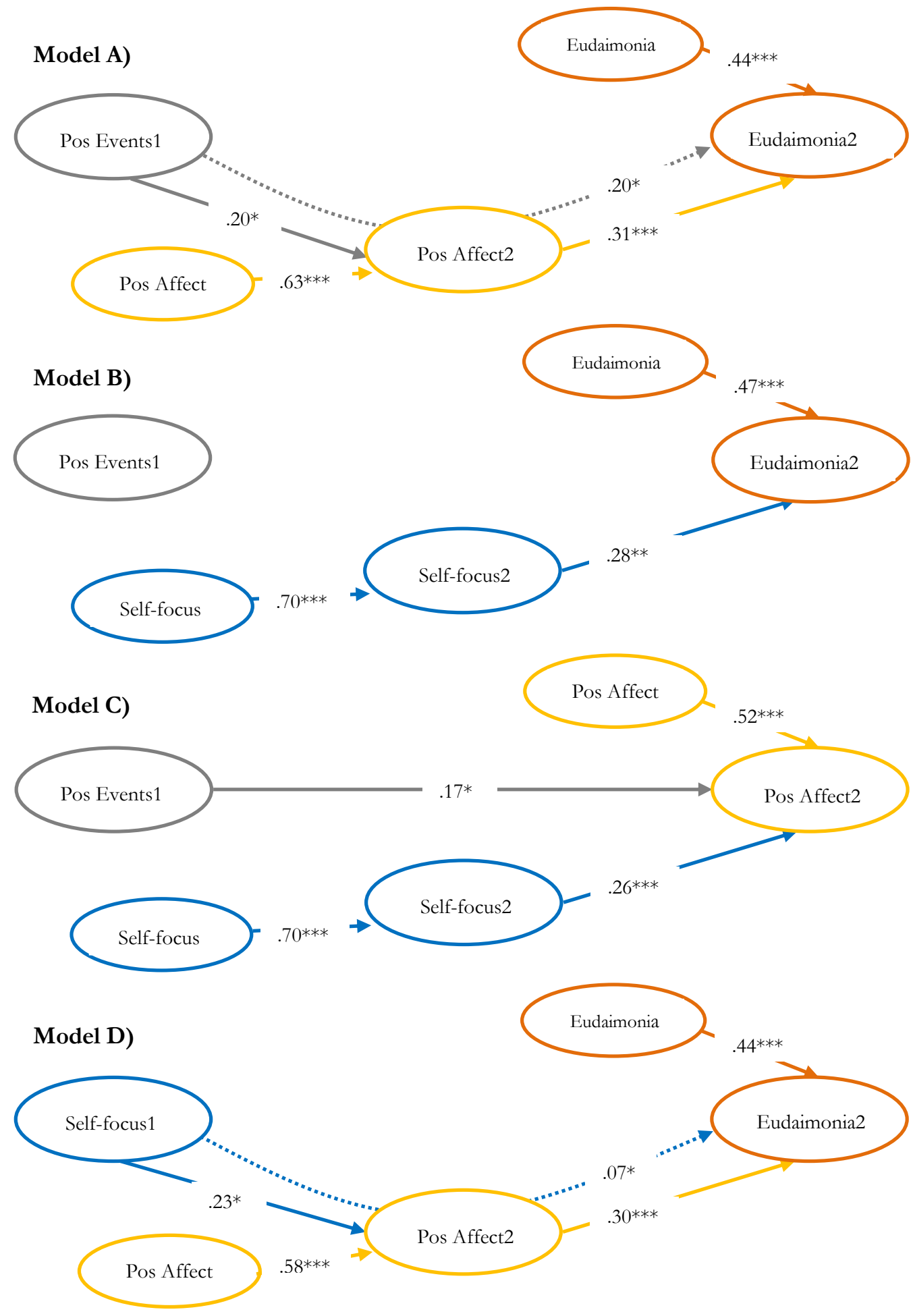

Figure 4.7. Summary of Longitudinal residualised mediational path models with mediators at Time 2. Note. The solid arrows and values indicate standardised direct relationships, and the dashed arrows and values indicate standardised indirect relationships. 
Hypothesis 10, baseline positive life events and eudaimonia at 4-weeks were mediated by positive affect at 4-weeks, a weak indirect effect $(\beta=.20$, se $=.03, \mathrm{CI}=.03-.11, p<.01)$ (Model A, Figure 4.7). Contrary to Hypothesis 9, baseline positive life events and eudaimonia at 4-weeks was not mediated by self-focused savoring at 4-weeks (Model B, Figure 4.7), nor was selffocused savoring a mediator of baseline positive life events and positive affect at 4-weeks (Model C, Figure 4.7). As predicted, however, by Hypothesis 10, positive affect at 4-weeks mediated the relationship from baseline self-focused savoring to eudaimonia at 4 -weeks $(\beta=.07$, se $=.06, \mathrm{CI}$ $=.07-.26, p<.05)($ Model D, Figure 4.7).

Gender moderation effects. The moderating effect of gender was explored on the final significant pruned mediational models, first with significant mediators at Time 1 then, with significant mediators at Time 2. Table 4.5 is a summary of the $\chi^{2}$, df, $\Delta \chi^{2}, \Delta \mathrm{df}$, and $p$ values for all significant residualised mediational models. First, gender was entered as the moderating

Table 4.5. Summary of Tests of Invariance by Gender.

\begin{tabular}{|c|c|c|c|c|c|}
\hline \multicolumn{6}{|c|}{ Significant Mediator Models with Mediators at Time 1} \\
\hline Model & $\chi^{2}$ & df & $\Delta \chi^{2}$ & $\Delta \mathrm{df}$ & $p$ \\
\hline \multicolumn{6}{|l|}{ Model A) PLE - PA - EUD } \\
\hline Baseline & 142.47 & 94 & - & - & - \\
\hline Paths Constrained & 142.57 & 96 & .10 & 2 & .95 \\
\hline \multicolumn{6}{|l|}{ Model C) PLE - SF - PA } \\
\hline Baseline & 130.01 & 94 & - & - & - \\
\hline Paths Constrained & 131.88 & 157 & .01 & 1 & .92 \\
\hline \multicolumn{6}{|l|}{ Model D) SF - PA - EUD } \\
\hline Baseline & 104.28 & 94 & - & - & - \\
\hline Paths Constrained & 107.18 & 96 & 2.90 & 2 & .24 \\
\hline \multicolumn{6}{|c|}{ Significant Mediator Models with Mediators at Time 2} \\
\hline Model & $\chi^{2}$ & df & $\Delta \chi^{2}$ & $\Delta \mathrm{df}$ & $p$ \\
\hline \multicolumn{6}{|l|}{ Model A) PLE - PA - EUD } \\
\hline Baseline & 210.36 & 156 & - & - & - \\
\hline Paths Constrained & 211.02 & 158 & 1.66 & 2 & .44 \\
\hline \multicolumn{6}{|l|}{ Model D) SF - PA - EUD } \\
\hline Baseline & 257.02 & 154 & - & - & - \\
\hline Paths Constrained & 257.07 & 156 & .05 & 2 & .98 \\
\hline
\end{tabular}

Note. $\mathrm{LA}=$ Low Arousal, $\mathrm{HA}=$ High Arousal, $\mathrm{SF}=$ Self-focus, and PA = Positive Affect. 
variable with no path constraints. Second, all paths between latent variables were constrained equal to test for invariance. The change in the chi square value and degrees of freedom from baseline to the fully constrained model was non-significant for all models. In other words, the interrelationships found between everyday positive life events, everyday self-focused savoring, positive affect, and eudaimonia over time were invariant between adolescent males and females, completing the confirmation of Hypothesis 11.

\section{Discussion}

This chapter further examined the psychometric properties of the abridged WOSC, as well as how savoring predicted and mediated components of wellbeing for adolescents over one month. Three broad research questions guided the current chapter: (1) Are the four factors of savoring consistent across time, stable over time, and relatively independent? (2) Are savoring strategies predictors of positive affect and eudaimonia over time, and is the relationship between savoring and positive affect bi-directional? and (3) Are there mediating relationships between positive life events, savoring, positive affect, and eudaimonia concurrently and over time? In addition, each of the three research questions included hypotheses for investigating the moderating effect of gender. The research questions and their related hypotheses are discussed in turn below.

\section{Hypotheses 1 through 4: Psychometric tests of savoring}

The first four hypotheses were upheld, attesting that the four-factor savoring structure is a reliable and valid measure for both male and female adolescents. The four savoring factors were confirmed at four weeks with confirmatory factor analysis, and the factor loadings, factor variances, and factor covariances were invariant across the four weeks. In addition, male and female adolescents shared the same four-factor structure of savoring, with similar factor loadings, factor variances, and factor covariances. The savoring test-retest reliabilities were moderate to high and each factor predicted itself across four weeks over and above predicting any other savoring factor, supporting Hypotheses 3 and 4.

\section{Hypotheses 5 through 8: Savoring strategies: Predictors of positive affect and eudaimonia}

Hypotheses 5 through 7 were not as consistently supported as the previous four hypotheses. Unexpectedly, high arousal savoring was unrelated to positive affect and selffocused savoring predicted positive affect but not eudaimonia over a month. The hypotheses were suggested by findings reported in Chapter 3. Notable differences between the two chapters existed, however, that may explain the unsupported aspects of the present hypotheses. The 
previous chapter observed concurrent relationships; this chapter chiefly observed longitudinal relationships. The previous chapter employed a measure of overall happiness; this chapter observed the frequency of positive affect over a month. Incomplete support of the hypotheses may in part be due to changes in the measurement of hedonic wellbeing, as well as the impact of time on the strength of relationships between savoring and wellbeing. Measurement differences aside, it appears that high arousal savoring, arguably a more fleeting type of savoring (i.e., jumping up and down and sharing the everyday event with someone), is unable to positively influence the frequency of positive affect over one month.

The identical measure of eudaimonia was used for both chapters; however, self-focus savoring was only able to predict eudaimonia concurrently in Chapter 3, not over a month in the present study. Self-focused savoring did predict positive affect as hypothesised. The more an adolescent counts their blessings, congratulates themselves, and considers how they will remember the event, the more they will feel good. Feeling good in turn influences adolescents' psychological and social wellbeing — a finding that was not hypothesised, although not unexpected (see Figure 4.1). This result hints at a mediational relationship where the impact of self-focused savoring on eudaimonia is mediated by positive affect. This mediational possibility was investigated under the third research question and will be discussed in the next subsection.

Dampening was found to negatively predict positive affect congruent with Hypothesis 6. Contrary to concurrent findings in Chapter 3 and Hypothesis 6, dampening was unrelated to eudaimonia a month later. The findings here indicate that if an adolescent thinks they are undeserving of a positive event, or that the positive event was substandard, they will feel emotionally worse a month later, but their psychological and social wellbeing will be unaffected. There is another possible mediational relationship indicated by the path model (Figure 4.1) where the negative impact of dampening on eudaimonia may be buffered by positive affect. This possibility was investigated under the third research question and found to be unsupported.

Contrary to the seventh hypothesis, self-focus savoring and positive affect did not bidirectionally influence one another. Only savoring led to positive affect over one month, not the other way around. As well as not supporting the hypothesis, this finding does not support the broaden-and-build theory in relation to savoring (Fredrickson, 1998, 2001). At least, no support was found here over one month's time. Savoring may still function as a mechanism within the broadening and building of positive emotions and resources in a bi-directional manner when shorter spans of time are observed with methods sensitive to momentary changes in affect and savoring (Bryant, et al., 2011). 
Lastly, each predictive relationship within the full path model (Figure 4.1) was invariant between adolescent males and females; put another way, males and females shared similar relationships between savoring and wellbeing across a month. Adolescents, regardless of their sex, similarly diminished their positive affect from dampening savoring a month prior. They similarly increased their positive affect from counting their blessings, congratulating themselves, and holding the memory of their positive event for future recall. They also similarly reported uplifts in their psychological and social wellbeing from their previously reported frequent positive affect the month before.

\section{Hypotheses 9 through 11: Savoring and positive affect: Concurrent and longitudinal mediators}

Everyday positive life events had no direct relationship with eudaimonia in either the concurrent model or residualised longitudinal models; therefore, the relationship from everyday positive life events to eudaimonia was fully mediated by savoring and positive affect where significant mediations were present. The results provided mixed support for Hypothesis 9, that savoring would be a mediator of the relationship between positive events and wellbeing both concurrently and over time. Self-focus savoring mediated concurrent relationships between everyday positive life events and positive affect and between everyday positive life events and eudaimonia. Similarly, baseline self-focus savoring mediated the relationship from baseline everyday positive life events to positive affect at 4-weeks; however, it did not mediate baseline positive events to eudaimonia at 4-weeks. Further, baseline positive events did not predict selffocus savoring at 4-weeks, therefore self-focus savoring was not a mediator from baseline positive events to either positive affect or eudaimonia at 4-weeks.

On the other hand, the results provided uniform support for Hypothesis 10 that positive affect would be a mediator of the relationship between positive events and wellbeing both concurrently and over time. Positive affect mediated the concurrent relationship between everyday positive life events and eudaimonia, although the strength of this mediation appeared weaker than the self-focus savoring mediation. Both baseline positive affect and positive affect at 4-weeks mediated the relationship between baseline everyday positive events and eudaimonia at 4-weeks. In addition, positive affect mediated, concurrently and over time, the relationship between self-focus savoring and eudaimonia regardless of whether positive affect was at baseline or 4-weeks.

Supporting the last hypothesis, there was no impact by gender on mediations at either one point in time or over the four weeks. The findings revealed that in the present moment, 
regardless of gender, the positive influence of positive events on feeling socially connected, having a sense of autonomy, purpose, and mastery depends on adolescents' ability to count their blessings, congratulate themselves and create positive memories, as well as feel pleasant emotions. Over time, however, self-focus savoring becomes ineffective as a mediator between positive events and eudaimonia, although savoring does mediate the relationship from positive events to positive affect over a month, if savoring and positive events are concurrent. Similarly for males and females, adolescents' self-focus savoring positively impacted eudaimonia a month later depending on the frequency of their positive affect; the more positive affect adolescents felt from self-focus savoring, the more connected and purposeful they felt. Positive affect consistently and fully mediated the relationship from positive events to eudaimonia, and from self-focus savoring to eudaimonia concurrently and over time for both males and females.

Overall, the psychometric evidence for the savoring scale was strong. Under stringent tests of its factor structure with CFA and tests of longitudinal invariance, the abridged WOSC conformed to the 4 factor model consistently and invariably over the four weeks. The internal reliability was replicated to be high, and the stability of dampening, low arousal, high arousal, and self-focused savoring was reasonably high across four weeks. The construct validity was shown to be robust as tests of cross-lag relationships between savoring strategies over one month were minimal. In addition, savoring was found to be a mediator of concurrent everyday positive events and eudaimonia, and a predictor of eudaimonia over time depending on adolescents' frequency of positive affect, regardless of gender.

The subsequent chapter seeks to replicate the psychometric evidence for the savoring scale, and further investigate longitudinal relationships between everyday positive events, savoring, and hedonic and eudaimonic wellbeing with adults. The present chapter investigated the influence of gender, rather than age, as it had a nearly equal gender ratio and little variation in age. The subsequent chapter returns to an examination of the influence of age on savoring and wellbeing, rather than gender, as it has a substantial age range and an imbalanced gender sample. 


\section{CHAPTER 5}

\section{Adult Savoring and Wellbeing over Time}

This chapter continued to investigate the psychometric properties of savoring and the direction of effect between savoring, wellbeing, and everyday positive life events in accordance with savoring theory. Compared with the previous chapter, the time frame for examining these relationships was expanded from one month to three months. Instead of adolescents, three broad age groups of adults were examined: young adults (16 to 30 years old), middle adults (31 to 49 years old), and older adults (50 to 88 years old).

In this last empirical chapter, a decision was made to focus on age over gender. An attempt to study both age and gender, or just gender, would mean the investigation of less complex questions. With gender as the focus, the data set would be reduced to less than half the original sample to create a more equal number of adult males and females, as over $80 \%$ of the sample was female. In addition, previous chapters indicated no to minimal gender differences for savoring. Chapter 4 found no adolescent gender differences for the structure of savoring or within the savoring and wellbeing relationships (although these findings may be different for adults). Few gender differences for adult savoring were found in Chapter 2; females showed higher high arousal and self-focus savoring compared to males. On the other hand, with age as the focus, the data set could retain its nearly 2,000 participants and allow the examination of more complex models, although the findings might be biased in favour of females and unable to account for gender differences. Since the objective of this thesis, however, was to observe potential developmental trends in savoring and wellbeing and since previous chapters found few effects by gender, I chose to retain the entire sample and focus on the demographic variable of age. This chapter thus moved away from evaluating the effect of gender on savoring and wellbeing to continue a more thorough investigation of the developmental assumptions and implications of savoring and wellbeing.

This chapter first sought to replicate the robust psychometric findings from Chapter 4 with the four-factor savoring measure. It tested whether differences by age for the covariances between dampening and the other three savoring strategies were replicated from Chapter 2. Then, differences between the age groups for each measure were investigated. It was expected that both dampening and high arousal savoring would decrease over adulthood. Previous research found that although the frequency of high arousal emotions may be relatively stable across adulthood, the intensity declines (Diener \& Suh, 1998; Pinquart, 2001). Likewise, savoring 
that is energetic and/or excited was anticipated to decline with age. Since the intensity of negative affect also decreases with age, it was proposed that dampening would decline with age. On the other hand, there appeared to be no decrease in low arousal emotions over time (Pinquart, 2001), thus, as was found in Chapter 2, no difference in low arousal savoring by age were expected. The last savoring strategy, self-focus savoring, is also expected to decrease with age under the same assumption supported by the previous study in Chapter 3 that high arousal positive emotions are less frequent and intense in older age. The emotions related to self-focus strategies like gratitude and pride may be both arousing and calming depending on many factors; however, they were assumed to be less arousing than excitement from high arousal savoring yet not as calming as a sense of serenity and peacefulness perhaps gained from low arousal savoring. Therefore, this study expected that self-focus savoring strategies would decrease with age, but perhaps not as clearly as high arousal would.

As with Chapter 3, the meaning orientation to happiness was used to measure eudaimonia (Peterson, et al., 2005). Slightly different to Chapter 3 that used overall happiness (Lyubomirsky \& Lepper, 1999), the present chapter introduced the pleasure orientation to happiness as a measure of hedonia. Peterson (et al., 2005) found young adults endorsed a more pleasure-orientated happiness, which was recently confirmed cross-culturally with a German sample (Ruch, Harzer, Proyer, Park, \& Peterson, 2011). Based on these findings, and research discussed in Chapter 3 (i.e. Carstensen, 2006; Carstensen, et al., 2010), it was expected that young adults would show an increase in pleasure orientation and decrease in meaning orientation compared to the older adults. No age differences were expected for global satisfaction, although components of satisfaction may have differed across the life span; for instance, although older adults would be expected to have more satisfaction in the present and less satisfaction toward the future than younger adults, global satisfaction shows little to no change over the adult lifespan (Carstensen, Pasupathi, Mayr, \& Nesselroade, 2000; Diener \& Suh, 1997; Kunzmann, Little, \& Smith, 2000; Lucas \& Gohm, 2000). Lastly, it was expected that the intensity of everyday positive events would decrease with age, similarly to the intensity of high arousal emotions (Pinquart, 2001). Further support for this assumption came from a recent study with older women $(N=101,63-93$ years old $)$ that found the reported frequency of positive events decreased with age (Charles, et al., 2010).

Of all the studies I have found researching orientations to happiness, none were longitudinal (Park, Peterson, \& Ruch, 2009; Peterson, et al., 2005; Peterson, et al., 2007; Ruch, et al., 2011; Vella-Brodrick, et al., 2009). (Granted, the study of the orientations to happiness, as 
conceptualised by Seligman and Peterson, has only been underway for less than a decade.) The correlational research indicated that orientations are highly and positively related at one point in time and that they each, and most robustly meaning orientation, predict satisfaction with life. The present study will be the first to my knowledge to explore pleasure and meaning orientations and satisfaction with life over time, and thus will be able to greatly expand our understanding of orientations to pleasure and meaning. In addition, it will illuminate their directional relationships with savoring and everyday positive events. It may be, for example, that although previous researchers assumed orientations predict satisfaction (e.g. Park, et al., 2009), satisfaction may in fact predict orientations — a possibility that will be studied here with longitudinal data.

Another relationship studied in this chapter was the predictive impact that everyday positive life events had on savoring. As Chapter 4 discovered, while the construct of everyday positive events was a predictor of positive affect, it was not a predictor of savoring over time. The theoretical assumption that savoring would result from positive events experienced a month prior was unfounded. Thus, this chapter does not make the same prediction as Chapter 4 - that positive events lead to savoring - and instead leaves the direction of effect between these two unspecified over three months. It is expected, however, that since positive events were unable to predict savoring across a month, they will be unable to predict savoring over three months; all other longitudinal relationships with everyday positive life events were exploratory. Further, it is expected that the larger path model will evidenced bi-directional relationships that will warrant testing the equality between directional paths.

\section{Hedonic and eudaimonic orientations to happiness as moderators of savoring}

Recall from the review of literature on the Ways of Savoring in Chapter 2 that personality was found to be associated with savoring strategies; in particular, Bryant and Veroff (2007) found that those university students with a disposition toward positive emotions had positive associations with all types of savoring strategies, except Kill-Joy Thinking. This study expected to find that international adults who were oriented toward pleasure (i.e. hedonia) would not only have positive associations with amplifying strategies but that they would also enhance savoring relationships. Previous research, however, has not investigated the association between a disposition toward meaning (i.e. eudaimonia) and savoring. This study was the first to my knowledge to explore their relationship. I expected that orientations toward both hedonia and eudaimonia would have positive concurrent and longitudinal relationships with amplifying savoring strategies. I also expected that hedonic and eudaimonic orientations would have an 
enhancing moderation effect on amplifying savoring relationships, and a buffering effect on dampening savoring relationships.

\section{Hypotheses: Adult Savoring and Wellbeing}

Four main aims guided analyses of adults' savoring and wellbeing indicators. These aims were: (1) to confirm the consistency, stability, and interrelationships between and among the four factors of adult everyday savoring that were found with adolescents; (2) to examine the impact of age on the structure of savoring, on savoring strategies, wellbeing indicators, and everyday positive events, and on the relationships between savoring and wellbeing over 3 months; and (3) to investigate the direction of effects between dampening, low arousal, high arousal, and selffocused savoring, and pleasure and meaning orientated happiness, satisfaction with life, and everyday positive life events; and (4) to investigate the moderating influence pleasure and meaning orientation to happiness had on savoring relationships. From these aims, I formed four research questions and thirteen related hypotheses.

(1) Are the four factors of adult savoring consistent across time, stable over time, and relatively independent as was found with adolescents?

Hypothesis 1: The four factors of savoring-dampening, low arousal, high arousal, and self-focused savoring - would be confirmed at Time 1 and at 3-months, and will be invariant across 3 months.

Hypothesis 2: The relationships between dampening and low arousal, dampening and high arousal, and dampening and self-focus savoring would be moderated by age, replicating findings from Chapter 2.

Hypothesis 3: Adults' dampening, low arousal, high arousal, and self-focused savoring would evidence moderate to high test-retest reliability over 3 months.

Hypothesis 4: Cross-lag relationships will be minimal over time and of less strength than stability coefficients, confirming longitudinal findings with adolescents and supporting the relative independence of each savoring strategy.

(2) Do adults at different stages of adulthood differ on savoring strategies, pleasure and meaning orientations to happiness, satisfaction with life, and everyday positive events?

Hypothesis 5: Younger adults would report more dampening, high arousal, and selffocus savoring, greater pleasure orientation, and more intense everyday positive events. Hypothesis 6: There would be no differences in low arousal savoring across ages in adulthood. 
Hypothesis 7: Older adults would report greater meaning orientation to happiness.

\section{(3) Do orientations to happiness predict savoring strategies, vice versa, or both?}

Hypothesis 8: Dampening savoring would be negatively related to satisfaction with life.

Hypothesis 9: Low arousal savoring would be positively related to meaning orientation.

Hypothesis 10: High arousal savoring would be positively related to pleasure orientation.

Hypothesis 11: Self-focus would be positively related to satisfaction with life.

\section{(1) Do orientations to happiness moderate savoring relationships?}

Hypothesis 12: Pleasure and meaning orientations to happiness would buffer dampening savoring relationships.

Hypothesis 13: Pleasure and meaning orientations to happiness would enhance amplifying savoring relationships.

\section{Method}

\section{Participants}

The total sample was composed of 1858 individuals ranging in age from 16 to 88 years ( $M=40 \mathrm{yrs}, 83 \%$ of the sample was comprised of females) taking part in the International Wellbeing Study (IWS) detailed in Chapter 2. ${ }^{29}$ Figure 5.1 depicts the distribution of age across the sample. The sample included 558 fifteen to thirty-year-olds (110 males, 448 females), 816

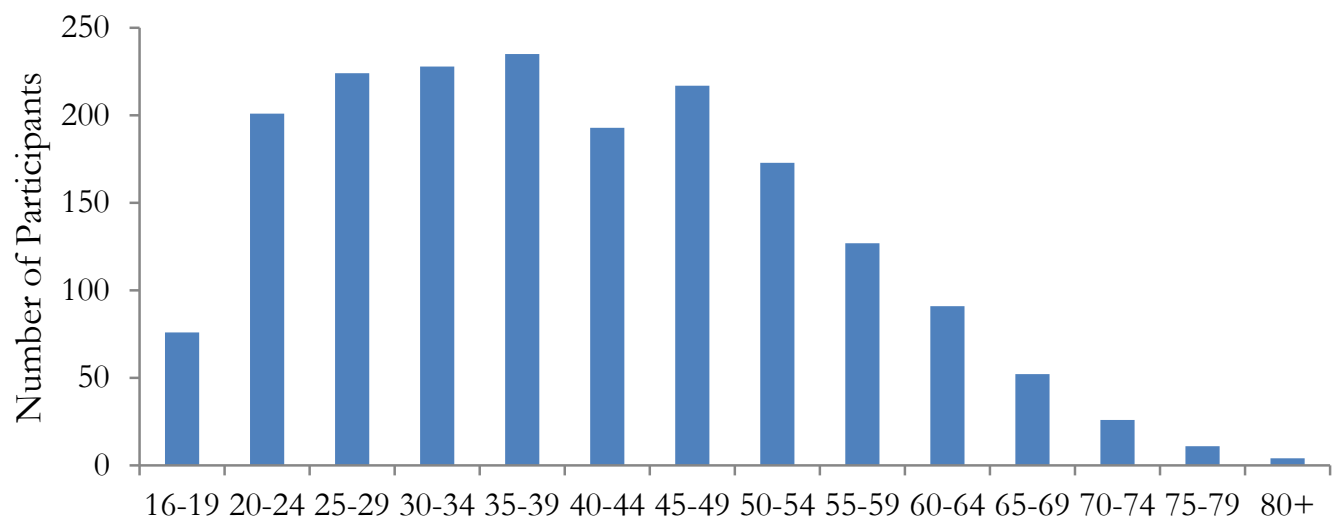

Age in years

Figure 5.1. Age distribution of the study sample.

${ }^{29}$ Recall from Chapter 2 that the International Wellbeing Study is an online 12-month longitudinal assessment study consisting of five consecutive assessment points. The project is running for four years, from March 2009 to March 2013, with rolling enrolment. 
thirty-one to forty-nine-year-olds (107 males, 709 females), and 484 fifty to eighty-eight-year-olds (96 males, 388 females). See Figure 5.1 for a distribution of age by five-year intervals up to 80 years of age. All participants were English speakers, with the majority from New Zealand (52\%), North America (24\%), Australia (10\%), and the United Kingdom (9\%). Smaller numbers were from Europe's mainland $(<4 \%)$ and Asia $(<2 \%)$. The ethnic majority was White or European (84\%), and a minority was Asian (6\%), Māori /Pasifika (3\%), Latin (1\%), and African (including African-American and African-European, $<1 \%$ ), with a similar variation in occupations as reported in Chapter 2.

\section{Procedure}

The adults selected for this chapter completed on-line surveys at two time points separated by three months. The present study aggregated all eight available cohorts from the rolling enrolment to comprise the largest number of individuals who had completed surveys at Times 1 and 2, the majority of which was received from the initial cohort (32\%). After individuals participated in their first survey, they were sent an automated email thanking them for their participation. An email reminder was sent to participants as their second time point was approaching to inform them that their second time-point survey was now available on-line, and another reminder was sent after two weeks if they had still not participated. If an individual failed to participate during their designated survey month, they were dropped from continuing the study.

\section{Measures}

Everyday Positive Life Events (PLE). A condensed version of the PLE scale (Jose, 2009) was used to measure individuals' perceived intensity of 7 everyday positive events that included 5 prescribed and 2 voluntarily elected events. Participants were asked whether an event (e.g. "You had an experience that was fun and exciting", "You got emotionally closer to someone", and "Your health or fitness improved") had happened over the last three months, 'yes' or 'no', and 'If you said 'yes', how much of a positive experience was it?' $(0=$ none; $1=$ a little; $2=$ some; $3=$ a lot). The intemal reliability for the intensity of positive events was moderately high at baseline $(\alpha$ $=.70)$, and it was similar at 3-months $(\alpha=.68)$. The test-retest reliability was not expected to be high and it turned out to be moderate $(r=.46, p<.001)$. Immediately after filling out the PLE, participants were asked how they responded (savored) to their everyday positive events.

Savoring. Cognitive and behavioural savoring strategies were again measured using the abridged 20-item version of the original 60-item Ways of Savoring Checklist (WOSC) (Bryant \& Veroff, 2007) originally described and investigated in Chapter 2. Answers were given along a 7- 
point Likert scale $(1=$ strongly disagree; $4=$ neutral; $7=$ strongly agree $)$ indicating how adults responded to positive events during the past three months. Twenty items were subjected to confirmatory factor analysis (CFA) previously reported in Chapter 2, revealing that a four-factor structure of savoring by using dampening, low arousal, high arousal, and self-focused savoring of everyday positive events fit the data well for adults $\left(\chi^{2} / \mathrm{df}=4.26, \mathrm{CFI}=.94\right.$, RMSEA $=.06$, $\operatorname{sRMR}=.04)$.

Orientations to Happiness. Individuals completed the Orientations to Happiness scale (Peterson, et al., 2005) which asked respondents to indicate "How you actually live your life" by providing a level of agreement to 18 items about three forms of happiness on a 5-point Likert scale $(1=$ not like me at all; $2=$ a little like me; $3=$ somewhat like me; $4=$ mostly like me; $5=$ very much like me). The three forms of happiness were: pleasure (6 items, e.g., "life is too short to postpone the pleasures it can provide"), engagement (6 items, e.g., "regardless of what I am doing, time passes very quickly"), and meaning (6 items, e.g., "my life serves a higher purpose”). The present study used only the pleasure and meaning orientations to happiness. Internal reliability was highest for meaning at both baseline $(\alpha=.84)$ and 3-months $(\alpha=.86)$, followed closely by pleasure at baseline $(\alpha=.81)$ and 3-months $(\alpha=.82)$. The test-retest reliabilities were high for both meaning $(r=.84, p<.001)$ and pleasure $(r=.79, p<.001)$.

Temporal Satisfaction with Life (TSWL). Individuals responded to the same 15-item Temporal Satisfaction with Life Scale (Pavot, et al., 1998) described in Chapter 3 for the adult sample. The TSWL can measure both global satisfaction, and satisfaction with the past, present, and future; a global score is achieved by combining judgements about an individual's satisfaction for the past (5 items), present (5 items), and future (5 items). The global level of satisfaction with life was used in the analyses reported here, in other words, the scores for past, present, and future satisfaction were combined to form a total satisfaction with life score. The internal reliability was identical at baseline and 3-months $(\alpha=.91)$. The test-retest reliability was also high $(r=.82, p<.001)$.

\section{Results}

\section{Analytic Strategy}

The first four hypotheses were tested following the same process outlined in Chapter 4 under the Analytic Strategy section. The one difference was that in this chapter, the effect of age rather than gender was examined in an attempt to replicate findings from Chapter 2 that the relationships between dampening and the other three savoring strategies differentiate with age. 
Next, I tested for age differences in each of the constructs averaged across three months with an Oneway ANOVA and a Tukey post-hoc test.

In the following analyses I tested the direction of effect between savoring, wellbeing, and positive events in AMOS (Arbuckle, 2009) in three steps: (1) by constructing measurement models at each time point, (2) by testing for stationarity, (3) by saturating the residualised path model, and (4) by pruning the model from least to most significant, up to a marginal p-value of .07 .

Subsequently, exploratory analyses were conducted on the bi-directional relationships within the model to test for invariance first within the path model and then in focussed path models. The focussed bi-directional path models were examined for invariance between the strength of bi-directional paths following the method outlined by Byrne (2001). The process of invariance testing will be briefly detailed in the relevant section of the results.

The last two hypotheses were tested with moderation analyses. Regressions were conducted on average orientation to pleasure and average orientation to meaning as moderators of the relationship from baseline savoring to everyday positive life events at 3-months. Further process details follows in the relevant section of the results.

\section{Confirmation of findings from adolescent savoring with adults: The reliability and validity of savoring.}

Consistent with all previous chapters, model goodness of fit was assessed by four criteria: the ratio of the chi square value divided by the degrees of freedom $\left(\chi^{2} / \mathrm{df}\right)$, the comparative fit index (CFI), the root mean square error of approximation (RMSEA), and the root mean square residual (sRMR) (Hu \& Bentler, 1999; Jöreskog \& Sörbom, 1982; Steiger, 1990). A good model consists of a ratio of less than $3-5$ for the $\chi^{2} / \mathrm{df}$, a CFI of .90 or above, a RMSEA below .08, and a sRMR below .06. For further details of these fit indices, please refer to the section on Factorial Validity in Chapter 2.

Confirmatory Factor Analysis. To evaluate the first hypothesis, that the four-factor savoring structure would be confirmed at both time points, a CFA was conducted at Time 1, then at Time 2 ( 3 months after Time 1). As with the previous chapters, dampening, low arousal, and self-focus savoring each were based on 3 items, and high arousal savoring was based on four, making a total of 13 items. The parameter to participant ratio was well above the minimal cut-off of 3 , it was 32 parameters per participant. At Time 1 , the initial model was adequate except for the chi square ratio which was high $\left(\chi^{2} / \mathrm{df}=7.69, \mathrm{CFI}=.93, \mathrm{RMSEA}=.06\right.$, SRMR $=.04)$. An observation of the Modification Indices (MI) indicated that allowing two sets of error 
terms to correlate on high arousal parcels would improve the model fit. The MI for the covariance between item 1 and item 9 error terms (both sharing with others items) was 85.77, and the MI for the covariance between item 4 and item 11 error terms (both behavioural expression items) was 72.51. After adding these covariance paths, the model fit improved for all indices except the sRMR: $\chi^{2} / \mathrm{df}=4.96, \mathrm{CFI}=.96, \mathrm{RMSEA}=.05, \mathrm{sRMR}=.04 .{ }^{30}$ All 13 items yielded significant loadings on their respective latent variable at the level of $p<.001$. The standardised regression weights for the factor loadings ranged from .25 to .78 with an average of .54. The correlation between dampening and high arousal savoring was non-significant. All other correlations between latent variables were significant at the level of $p<.001$ and ranged from .20 to .89. Table 5.1 presents the latent variable correlations. The correlation between dampening

Table 5.1. Summary of Correlations between the Four Factors of Everyday Savoring.

\begin{tabular}{lcccc}
\hline & Dampening & Low Arousal & High Arousal & Self-focus \\
\hline Dampening & - & $.32^{* * *}$ & $.01^{\mathrm{NS}}$ & $.20^{* * *}$ \\
Low Arousal & $.34^{* * *}$ & - & $.70^{* * *}$ & $.72^{* * *}$ \\
High Arousal & $-.03^{\mathrm{NS}}$ & $.70^{* * *}$ & - & $.89^{* * *}$ \\
Self-focus & $.13^{* * *}$ & $.69^{* * *}$ & $.84^{* * *}$ & - \\
\hline Note. Time 1 correlations are above the diagonal, and Time 2 correlations are below the diagonal. ${ }^{* * *} p<.001$.
\end{tabular}

and self-focus savoring was the weakest, dampening and low arousal savoring shared a slightly stronger relationship. A strong relationship was found between low arousal and high arousal savoring, and between low arousal and self-focus savoring with the strongest correlation found between high arousal and self-focus savoring.

A CFA at 3-months was then modelled based on the post-hoc Time 1 model (including the two post-hoc error covariances), returning a good model fit except for the chi square degrees of freedom ratio: $\chi^{2} / \mathrm{df}=6.30, \mathrm{CFI}=.95, \mathrm{RMSEA}=.05$, $\mathrm{RMR}=.04 .{ }^{31}$ As with the Time 1 post-

\footnotetext{
${ }^{30}$ The $\chi^{2} / \mathrm{df}$ was below the lineate cut-off of 5 for the post-hoc model, but not below the more stringent cut-off of 3. The post-hoc model was run with a random sample of 500 participants, from the original data-set, to test the possibility that the large sample size was inflating the $\chi^{2} / \mathrm{df}$. With the smaller sample, the $\chi^{2} / \mathrm{df}$ was 2.32 and all other fit indices remained identical supporting the assertion that the $\chi^{2} / \mathrm{df}$ may have been adversely affected by the larger sample of 1858 participants.

${ }^{31}$ The Time 2 model was also run with the random sample of 500 participants, which again improved the $\chi^{2} / \mathrm{df}$ ratio $\left(\chi^{2} / \mathrm{df}=3.14\right)$ and all other fit indices remained the same.
} 
hoc model, all 13 items yielded significant loadings on their respective latent variable at the level of $p<.001$. The standardised regression weights for factor loadings ranged from .29 to .78 with an average of .56. The correlation between dampening and high arousal savoring was again not significant. All other correlations between latent variables were significant at the level of $p<.001$ and ranged from .13 to .84 in the same order-from weakest to strongest—as the latent variable correlations for the Time $1 \mathrm{CFA}$ model, refer to Table 5.1. In concluding the CFA analyses, the four-factor savoring model was a good representation of the data at Time 1 and three months later, supporting the first hypothesis.

Stationarity. Chapter 4 found that adolescent savoring was stationary after one month. Stationarity is the degree to which relations among variables are stable across time (Kenny, 1979; MacKinnon, 2008). This question of stationarity was extended to adults by further testing Hypothesis 1 that savoring would be stationary, i.e. invariant, after three months.

The baseline model allowed all the relationships among savoring variables for both study waves to be freely estimated, including factor loadings, variances, and covariances; whereas, the fully constrained model assumed these relationships were equal between study waves. If the difference in chi square value and degrees of freedom between the baseline and fully constrained model was significant, then the models would not be stationary. On the contrary, if there was no significant change in the chi square value and degrees of freedom between study waves then the model was considered stationary. The chi square value and degrees of freedom for the baseline model $\left(\chi^{2}=619.26, \mathrm{df}=110\right)$ and the fully constrained model $\left(\chi^{2}=635.87, \mathrm{df}=131\right)$ did not significantly differ $\left(\Delta \chi^{2}=16.61, \Delta \mathrm{df}=21, p=.74\right)$, supporting the sufficient stationarity of the four factor savoring model (Hypothesis 1).

Age moderation effects. Chapter 2 found differences in the covariances between dampening and low arousal savoring, dampening and high arousal savoring, and dampening and self-focus savoring between adolescents and adults. Adolescents' dampening had a significantly stronger relationship with each of the other savoring strategies than it did for adults. The present chapter investigated this possible developmental trend further with three age groups: young adults (16 to 30), middle adults (31 to 49), and older adults (50 to 88) at Time 1 followed by Time 2. The findings partially supported Hypothesis 2.

An omnibus test of the dampening covariances across the three age groups at Time 1 revealed a statistically significant difference (Model A2, Table 5.2). Subsequent tests were then 
Table 5.2. Time 1 Goodness-of-Fit Statistics for Tests of Invariance of Dampening Covariances across Young, Middle, and Older Adults.

\begin{tabular}{|c|c|c|c|c|c|c|}
\hline & Model Description & $\chi^{2}$ & df & $\Delta \chi^{2}$ & $\Delta \mathrm{df}$ & pvalue \\
\hline A1 & $\begin{array}{l}\text { Combined baseline models } \\
\text { (All Adults) }\end{array}$ & 444.55 & 165 & - & - & - \\
\hline A2 & $\begin{array}{l}\text { All dampening covariances } \\
\text { constrained equal }\end{array}$ & 461.03 & 171 & 16.48 & 6 & $p<.05$ \\
\hline B1 & $\begin{array}{l}\text { Combined baseline models } \\
\text { (Young and Middle Adults) }\end{array}$ & 277.29 & 110 & - & - & - \\
\hline B2 & $\begin{array}{l}\text { All dampening covariances } \\
\text { constrained equal }\end{array}$ & 292.46 & 113 & 15.17 & 3 & $p<.01$ \\
\hline B3 & $\begin{array}{l}\text { Dampening/Low Arousal } \\
\text { covariance constrained }\end{array}$ & 281.35 & 111 & 4.06 & 1 & $p<.05$ \\
\hline B4 & $\begin{array}{l}\text { Dampening/High Arousal } \\
\text { covariance constrained }\end{array}$ & 281.04 & 111 & 3.75 & 1 & NS \\
\hline B5 & $\begin{array}{l}\text { Dampening/Self-focused } \\
\text { covariance constrained }\end{array}$ & 280.01 & 111 & 2.72 & 1 & NS \\
\hline C1 & $\begin{array}{l}\text { Combined baseline models } \\
\text { (Young and Older Adults) }\end{array}$ & 274.33 & 110 & - & - & - \\
\hline C2 & $\begin{array}{l}\text { All dampening covariances } \\
\text { constrained equal }\end{array}$ & 279.98 & 110 & 6.65 & 3 & NS \\
\hline D1 & $\begin{array}{l}\text { Combined baseline models } \\
\text { (Middle and Older Adults) }\end{array}$ & 337.46 & 110 & - & - & - \\
\hline D2 & $\begin{array}{l}\text { All dampening covariances } \\
\text { constrained equal }\end{array}$ & 338.31 & 113 & .85 & 3 & NS \\
\hline
\end{tabular}

Note. $\Delta \mathrm{X}^{2}$, difference in $\mathrm{X}^{2}$ values; $\Delta \mathrm{df}$, difference in degrees of freedom. Models $\mathrm{A} 1$ - A2 includes all age groups, Models B1 - B5 compares Young and Middle adults, Models C1 - C2 compares Young and Older adults, and Models D1 - D2 compares Middle and Older adults.

conducted by pairing two age groups at a time. First, young and middle adults were compared, then young and older adults, and lastly middle and older adults. The baseline model for young and middle adults was compared to the constrained model, revealing a significant difference (Model B2, Table 5.2). Next, only the dampening - low arousal covariance for young and middle adults was constrained, revealing a significant difference (Model C2, Table 5.2). This constraint was released and the dampening - high arousal covariance was constrained, which turned out to be non-significant (Model D2, Table 5.2). The covariance between dampening and high arousal was then released and then the covariance between dampening and self-focus was constrained, which also returned a non-significant change in the $\chi^{2}$ (Model D2, Table 5.2). The baseline model for young and older adults was compared to the constrained model with no significant 
change in $\chi^{2}$ (Model B3, Table 5.2). Similarly, middle and older adults evidenced invariant covariances between dampening and low arousal, high arousal, and self-focus savoring (Model B5, Table 5.2).

The same process of invariance testing for the dampening covariances was implemented at Time 2 and confirmed the Time 1 results, see Figure 5.2. Only young and middle adults

Time 1

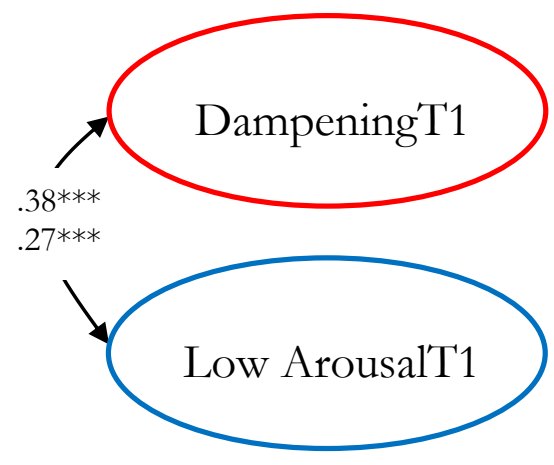

Time2

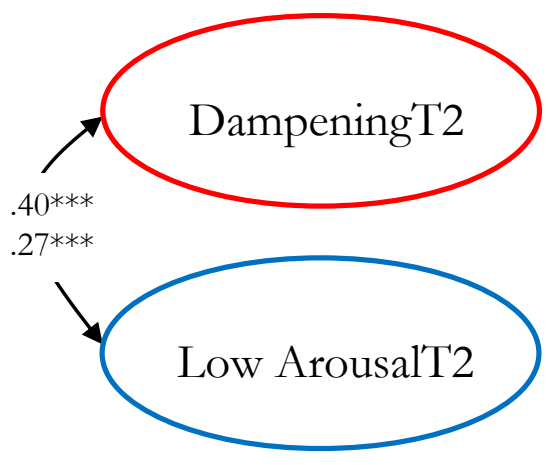

Figure 5.2. The correlations between dampening and low arousal savoring at Time 1 (left model) and Time 2 (right model) for young (top value) and middle aged adults (bottom value).

differed and again only for the covariance between dampening and low arousal, revealed by the difference between baseline $\left(\chi^{2}=325.67, \mathrm{df}=110\right)$ and constrained models $\left(\chi^{2}=332.15, \mathrm{df}=\right.$ 111; $\left.\Delta \chi^{2}=6.48, \Delta \mathrm{df}=1, p<.05\right)$. The tests of invariance, in summary, partially supported Hypothesis 2. Similar to adolescents in Chapter 2, the young adults here held a stronger association between their dampening and low arousal savoring than their slightly older counterpart, middle adults. The adolescents in Chapter 2 evidenced the strongest correlation $(.72, p<.001)$ between dampening and low arousal savoring, followed here by young adults and lastly, middle adults. It appears that these two savoring strategies become more distinct with age, from adolescents through middle age. Interestingly, older adults were not significantly different to either young or middle adults, indicating that dampening and low arousal savoring begin to again be more associated in the later years of life. Hypothesis 2 was only partially supported as the present results evidenced fewer differences than were previously found between adolescents and adults in Chapter 2.

Test-retest Reliability and Internal Validity. Chapter 4 found adolescent savoring to be highly stable over a month, and found limited interrelationships between savoring factors over time. The results in this chapter were expected to parallel these findings by confirming the 
hypotheses that adults' dampening, low arousal, high arousal, and self-focus savoring strategies are reliable over time and internally valid (Hypothesis 3 and 4). To answer questions about both reliability and validity, a two-step approach was adopted. First, to test the stability of savoring strategies, the data from Times 1 and 2 were subjected to path analysis in AMOS (Arbuckle, 2009), where direct paths from individual Time 1 variables only predicted themselves 3 months later (i.e. at Time 2). Second, the model was fully saturated by adding all possible cross-lags to the stability model. Then the cross-lag model was pruned from the least to most significant cross-lag to determine the degree of overlap among the four savoring strategies over 3 months.

Supporting the third hypothesis, adult savoring strategies across 3 months returned an adequate model fit $\left(\chi^{2} / \mathrm{df}=3.32, \mathrm{CFI}=.96, \mathrm{RMSEA}=.04, \mathrm{sRMR}=.03\right)$, and all four stability coefficients were significant at the level of $p<.001$ with moderately high standardised stability coefficients: dampening $(\beta=.58)$, low arousal $(\beta=.60)$, high arousal $(\beta=.69)$, and self-focus savoring $(\beta=$.66). To explore the fourth hypothesis, the exploratory baseline cross-lag model allowed each latent variable at Time 1 to predict each latent variable at 3-months composing a total of 16 structural paths, including 4 stability paths. The saturated model fit the data adequately $\left(\chi^{2} / \mathrm{df}=3.35, \mathrm{CFI}=.96, \mathrm{RMSEA}=.04\right.$, sRMR $\left.=.03\right)$. One marginally significant crosslag from self-focus savoring to high arousal savoring was revealed $(\beta=-.42, p=.05)$. The remaining cross-lags were non-significant and were individually pruned from least to most significant (up to $p<.05$ ). At each stage of the pruning process the beta weights, phis, and error terms were assessed to ensure multicollinearity was not a significant problem. The pruning process took 11 steps. The cross-lag from self-focus savoring at Time 1 to high arousal savoring three months later became highly significant $(\beta=-.42, p<.001)$. In the process, however, the high arousal standardised stability coefficient increased to 1.02, indicating high arousal at three months was being overpredicted. To control for this effect, I restricted the high arousal unstandardised stability coefficient to .56 , the same value found in the test-retest model. In the cross-lag model, however, this value translated to a slightly higher standardised value (.77) than what was found in the test-retest model (.69). No other cross-lags were revealed, and all stability coefficients remained highly significant $(p<.001)$, confirming Hypothesis 4 that cross-lags between savoring strategies would be minimal. The final pruned and post-hoc model improved the $\chi^{2} / \mathrm{df}$ ratio (3.29) and all other indices remained identical.

The adult four-factor savoring model appeared to be moderately to highly stable over three months, similar to adolescents (Chapter 4). It is worth noting that high arousal and selffocus savoring were the most highly correlated variables concurrently, although self-focus 
savoring negatively predicted high arousal savoring over three months, also similar to adolescents (recall that adolescent high arousal savoring negatively predicted self-focus savoring, however).

Developmental trends in savoring, hedonic and eudaimonic orientations to happiness, satisfaction with life, and everyday positive life events.

An Oneway ANOVA was conducted to explore differences by age in averaged savoring, happiness, satisfaction, and daily positive events across the three months. ${ }^{32}$ Three groups of adults $(16-30,31-49$, and $50-88$ years old) were compared on their average responses for dampening, low arousal, high arousal and self-focus savoring, pleasure and meaning orientations to happiness, satisfaction with life, and everyday positive events across Time 1 and Time 2.

Means and standard deviations for each measure and by each age group are shown in Table 5.3.

Table 5.3. Means and Standard Deviations for Savoring, Orientations to Pleasure and Meaning, Satisfaction with Life, and Everyday Positive Life Events Averaged across 3 Months for Young, Middle, and Older Adults.

\begin{tabular}{lcccccccc}
\hline \multirow{2}{*}{ Measure } & \multicolumn{2}{c}{$\begin{array}{c}\text { Young Adult } \\
\text { (15 to 30 years) }\end{array}$} & \multicolumn{2}{c}{$\begin{array}{c}\text { Middle Adult } \\
\text { (31 to 49 years) }\end{array}$} & \multicolumn{2}{c}{$\begin{array}{c}\text { Older Adult } \\
\text { (50 to 88 years) }\end{array}$} & \multicolumn{2}{c}{ Total } \\
\hline \multirow{2}{*}{ Dampening } & $\mathrm{M}$ & $\mathrm{SD}$ & $\mathrm{M}$ & $\mathrm{SD}$ & $\mathrm{M}$ & $\mathrm{SD}$ & $\mathrm{M}$ & $\mathrm{SD}$ \\
\cline { 2 - 10 } LowArousal & 3.07 & 1.26 & 2.96 & 1.13 & 2.72 & 1.12 & 2.93 & 1.17 \\
HighArousal & 5.81 & 1.16 & 3.94 & 1.05 & 3.86 & 1.12 & 3.88 & 1.11 \\
Self-focus & 4.72 & 1.01 & 5.03 & 1.02 & 4.84 & 1.12 & 5.04 & 1.05 \\
Pleasure & 2.94 & .80 & 2.80 & .79 & 2.75 & .80 & 2.83 & .79 \\
Meaning & 3.20 & .90 & 3.31 & .92 & 3.41 & .95 & 3.31 & .93 \\
Satisfaction & 4.41 & 1.08 & 4.34 & 1.08 & 4.46 & 1.06 & 4.39 & 1.07 \\
Pos. Events & 2.97 & 0.86 & 2.83 & .86 & 2.69 & .89 & 2.83 & .87 \\
\hline
\end{tabular}

The univariate effect by age was significant for six of the eight observed variables. Although all the effect sizes were small, age significantly predicted differences in dampening savoring, $F(2$, $1857)=12.12, p<.001$, partial $\eta^{2}=.03$, high arousal savoring, $F(2,1857)=17.60, p<.001$, partial $\eta^{2}=.02$, self-focus savoring, $F(2,1857)=3.42, p<.05$, partial $\eta^{2}<.01$, pleasure orientation to happiness, $F(2,1857)=8.37, p<.001$, partial $\eta^{2}=.01$, meaning orientation to happiness, $F(2,1857)=6.67, p<.01$, partial $\eta^{2}=.01$, and everyday positive events, $F(2,1857)=$

\footnotetext{
32 An analysis of time was not included since the savoring model, under stringent analysis, was stationary across the three months (refer to the sub-section on stationarity within the Results section of the present chapter).
} 
12.95, $p<.001$, partial $\eta^{2}=.01$. Tukey post hoc tests were observed for each significant univariate effect to ascertain which, and how, age groups differed on each measure. Figure 5.3 illustrates the reported rates of each of the eight measures, including those with non-significant differences, for young, middle, and older adults.

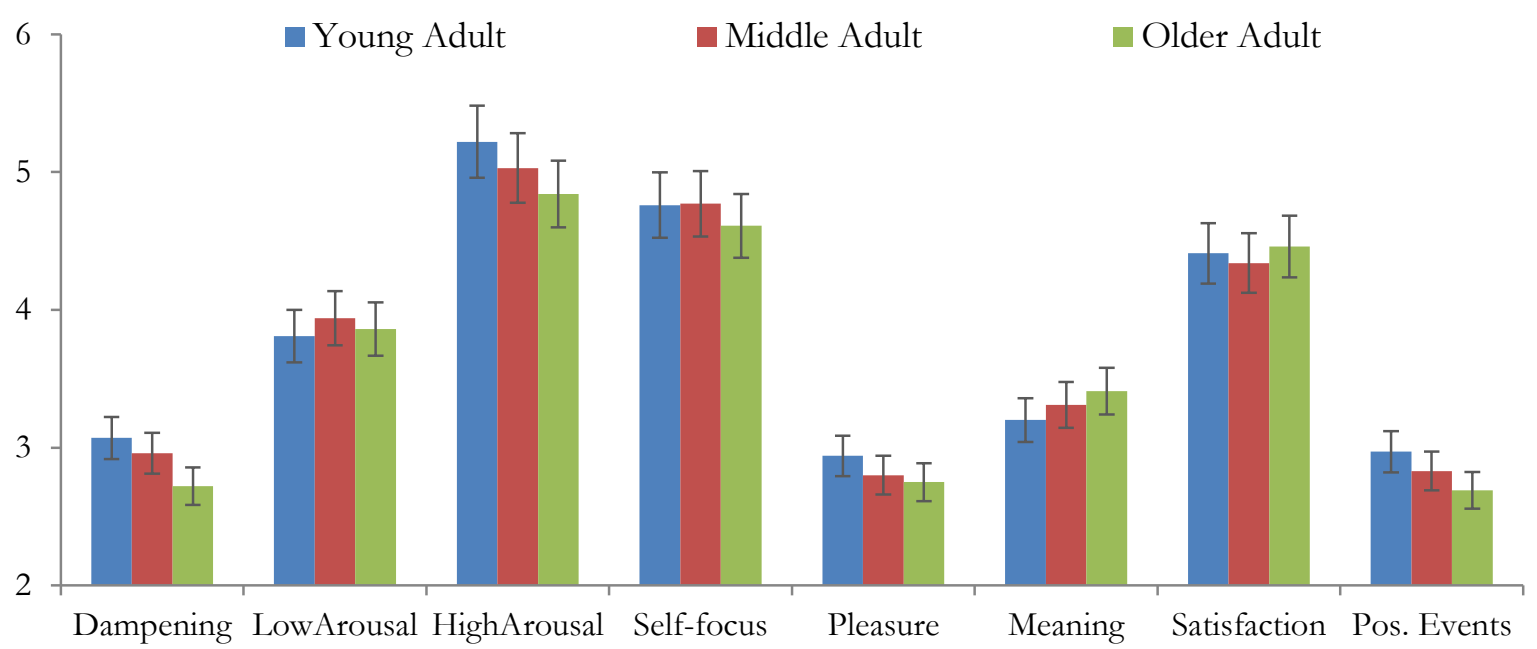

Figure 5.3. Reported rates of everyday savoring strategies, pleasure and meaning orientations to happiness, satisfaction with life, and everyday positive events by young (16 - 30 yrs), middle (31 -49 yrs), and older adults (50 - 88 yrs).

In support of Hypothesis 5, the Tukey post hoc test revealed young adults and middle adults endorsed dampening savoring strategies more than older adults $(p<.001$ and $p<.01$ respectively). Young and middle adults' mean dampening savoring was not significantly different. As hypothesised by Hypothesis 6, no significant difference in low arousal savoring by age $(F(2$, $1857)=2.307, p=.10$, partial $\eta^{2}<.01$ ) existed. In line with Hypothesis 5, high arousal savoring was the highest among young adults and linearly and significantly decreased at each stage of adulthood from $16-30$ years of age to $31-49$ years of age $(\not<.01)$, to $50-88$ years of age $(p$ $<.001)$. In addition, the decrease in high arousal savoring between $31-49$ years of age to $50-$ 88 years of age was also significant at $p<.01$. Middle adults reported significantly higher levels of self-focus savoring than older adults $(\phi<.05)$, and marginally higher than young adults $(p=.08)$, but there was no difference between young and older adults for self-focus savoring, partially supporting Hypothesis 5.

As stated by Hypothesis 5, results indicated young adults were more pleasure oriented than both middle $(p<.01)$ and older adults $(p<.001)$, and there was no significant difference 
between middle and older adults. Supporting Hypothesis 7, older adults were more meaning oriented than younger adults $(\phi<.01)$, but not more than middle adults, and middle adults were marginally more meaning oriented than young adults $(p=.09)$. Lastly, each age group significantly and linearly differed in their reported intensity of everyday positive life events. Young adults reported the highest intensity of everyday positive life events, higher than both middle $(p<.05)$ and older adults $(p<.001)$, and middle adults reported a higher intensity of positive events than older adults $(p<.05)$. That aging coincided with a significant linear decrease in everyday positive events supported Hypothesis 5.

\section{The directions of effect between savoring, hedonic and eudaimonic orientations to} happiness, satisfaction with life, and everyday positive life events across three months.

Measurement Models. To test Hypotheses 8 through 11, measurement models were constructed and tested before testing path models. First, three parcels each were designated for the orientation to pleasure (i.e. hedonia), and the orientation to meaning to happiness (i.e. eudaimonia) by sequentially selecting items for each parcel. The first item from the pleasure subscale of the orientations to happiness measure, for instance, was assigned to parcel 1, item 2 to parcel 2, item 3 to parcel 3, item 4 to parcel 1, and this pattern was continued until all pleasure items were parcelled. The same process was used for the items and parcels for everyday positive life events. Alternatively, the three parcels for satisfaction with life were the items corresponding to its three subscales: past, present, and future satisfaction with life. In other words, all the past satisfaction items were combined into parcel 1, present satisfaction items went into parcel 2, and future satisfaction items went into parcel 3.

Orientation to pleasure and meaning, satisfaction with life, and everyday positive events were then modelled with the four-factor savoring model at baseline and at 3-months. The participant to parameter ratio was very good at 16 participants per parameter. Both measurement models yielded a good fit to the data, although the baseline model had a slightly better fit, $\chi^{2} / \mathrm{df}$ $\left(\chi^{2} / \mathrm{df}=4.75, \mathrm{CFI}=.93, \mathrm{RMSEA}=.05, \mathrm{sRMR}=.04\right)$ than the 3 -month model, which had a better $\mathrm{CFI}\left(\chi^{2} / \mathrm{df}=4.84, \mathrm{CFI}=.94, \mathrm{RMSEA}=.05, \mathrm{sRMR}=.04\right)$. All factor loadings at baseline and 3months were highly significant, $p<.001$.

The average standardised regression coefficient for all factor loadings at baseline was .67, and ranged from .27 to 88 . At 3-months, the average standardised regression coefficient for all factor loadings was .66, and ranged from .30 to .88 . The overall mean squared multiple correlation was identical for baseline and 3-months (.47), indicating an acceptable level of 
criterion validity for the measurement model. The correlations between concurrent variables at baseline and at 3-months are reported in Table 5.4.

Table 5.4. Correlations among Latent V ariables for Baseline and 3-month Measurement Models.

\begin{tabular}{|c|c|c|c|c|c|c|c|c|}
\hline & Damp & LA & $\mathrm{HA}$ & SF & Pleasure & Meaning & SWL & EPLE \\
\hline Damp & - & $.32 * * *$ & $.01 \mathrm{~ns}$ & $.19^{* * *}$ & $.06^{*}$ & $-.07 *$ & $-.21 * * *$ & $-.01 \mathrm{~ns}$ \\
\hline LA & $.34 * * *$ & - & $.70^{* * *}$ & $.72^{* * *}$ & $.34 * * *$ & $.31 * * *$ & $.22^{* * *}$ & $.35^{* * *}$ \\
\hline HA & $-.03^{\mathrm{ns}}$ & $.66^{* * *}$ & - & $.88^{* * *}$ & $.43^{* * *}$ & $.29 * * *$ & $.40^{* * *}$ & $.55^{* * *}$ \\
\hline SF & $.13^{* *}$ & $.69 * * *$ & $.84 * * *$ & - & $.41 * * *$ & $.36 * * *$ & $.40^{* * *}$ & $.51 * * *$ \\
\hline Pleasure & $.09 * *$ & $.37 * * *$ & $.44 * * *$ & $.41 * * *$ & - & $.29 * * *$ & $.36^{* * *}$ & $.42 * * *$ \\
\hline Meaning & $-.10 * * *$ & $.29 * * *$ & $.31 * * *$ & $.35^{* * *}$ & $.25^{* * *}$ & - & $.35^{* * *}$ & $.25^{* * *}$ \\
\hline SWL & $-.24 * * *$ & $.29 * * *$ & $.37 * * *$ & $.41 * * *$ & $.36^{* * *}$ & $.35^{* * *}$ & - & $.42 * * *$ \\
\hline EPLE & $-.05^{\mathrm{ns}}$ & $.34 * * *$ & $.49 * * *$ & $.46^{* * *}$ & $.38 * * *$ & $.23^{* * *}$ & $.40^{* * *}$ & - \\
\hline
\end{tabular}

Note. Damp = Dampening, LA = Low Arousal, HA = High Arousal, SF = Self-focus, Pleasure = Orientation to Happiness

Pleasure, Meaning = Orientation to Happiness Meaning, SWL = Satisfaction with Life, and EPLE = Everyday Positive Life

Events. Values above the diagonal represent baseline correlations; values below the diagonal represent correlations at 3-

months. ${ }^{* * *} p<.001,{ }^{* *} p<.01, * p<.05,{ }^{*}$ ss $p>.10$.

Structural model over time. After finding that the measurement models were acceptable, a structural path model was drawn. The only savoring strategy stipulated to predict another savoring strategy was from self-focus to high arousal savoring, based on findings from the cross-lag analysis. The remainder of the model was fully saturated. During model refinement, paths were pruned from least to most significant until all paths reached at least marginal significance, $p \leq .10$ (Byrne, 2001; Kline, 2005).

A residualised longitudinal path model was constructed with eight variables at Time 1 and at Time 2: dampening, low arousal, high arousal, self-focus, pleasure and meaning orientations to happiness, satisfaction with life, and everyday positive life events. All eight latent variables were allowed to correlate concurrently, as indicated by the majority of moderate to high significant correlations from the measurement models (Table 5.4). Error terms for each of the eight variables at Time 1 were correlated with their respective error term at Time 2 , and stability paths were stipulated for each of the eight variables across the three months. In total, 53 structural paths were stipulated including the eight stability paths. After pruning, the final model supported a total of 25 structural path remained, including the eight stability paths, Figure 5.4. 


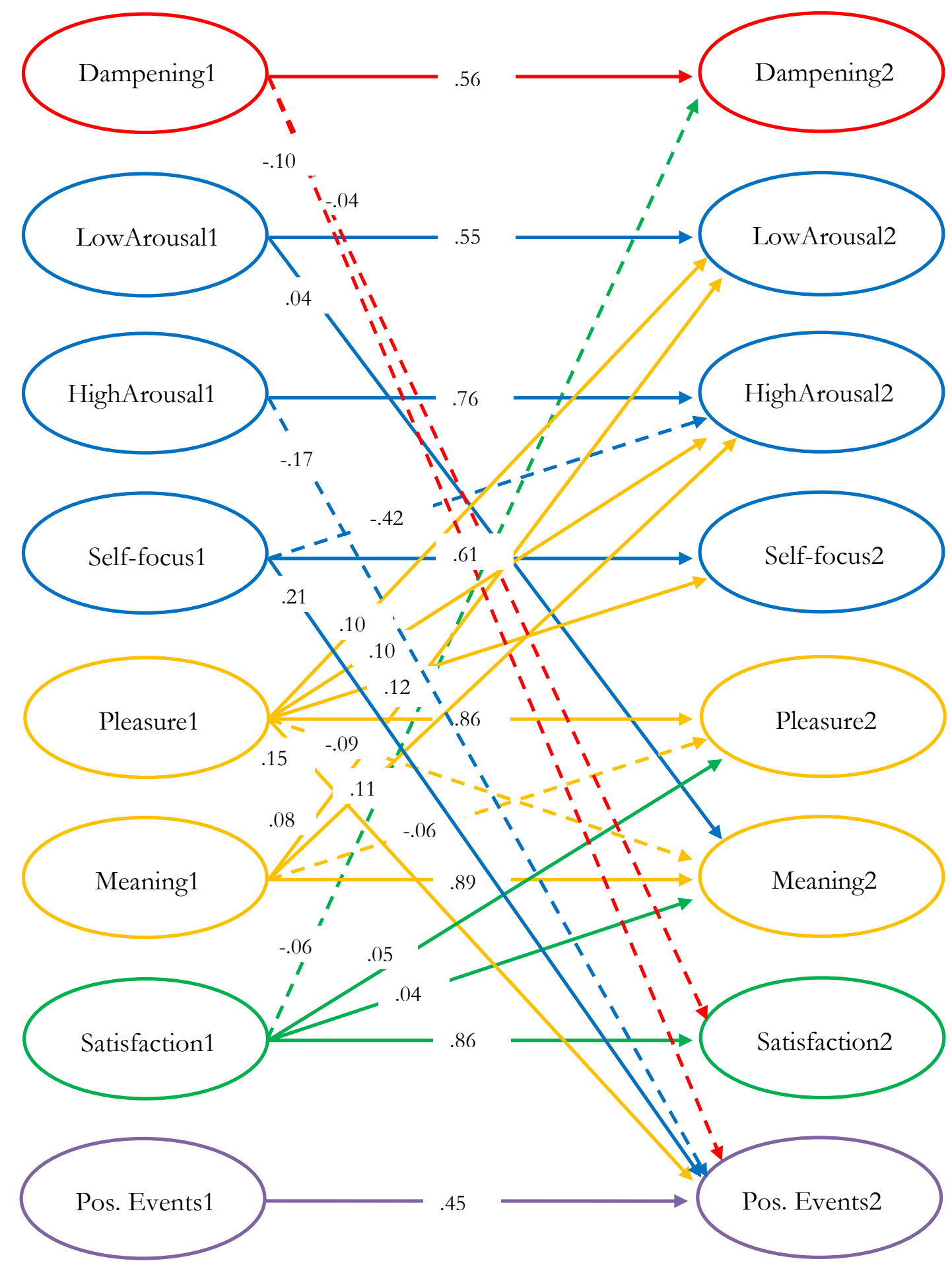

Figure 5.4. Pruned direction of effect model: Savoring, pleasure and meaning orientations to happiness, satisfaction with life, and everyday positive events across three months $\left(\chi^{2} / \mathrm{df}\right.$ ratio $=$ $2.74, \mathrm{CFI}=.96, \mathrm{RMSEA}=.03, \mathrm{sRMR}=.04)$. Note. Solid lines represent positive relationships and dashed lines represent negative relationships. 
The base model yielded an adequate fit to the data $\left(\chi^{2} / \mathrm{df}=2.80, \mathrm{CFI}=.96\right.$, RMSEA $=.03$, $\operatorname{sRMR}=.04)$ with a total of 315 parameters that provided an acceptable participant to parameter ratio of 6:1. All stability paths were significant at the level of $p<.001$, as was the cross-lag from self-focus to high arousal savoring. Seven cross-lag paths were also significant at the level of $p<$ .05: pleasure orientation to satisfaction $(\beta=-.02, p<.05)$, pleasure to high arousal $(\beta=.07, p<$ $.05)$, pleasure to low arousal savoring $(\beta=.09, p<.05)$, meaning orientation to low arousal $(\beta=$ $.09, p<.01)$, meaning to pleasure $(\beta=-.06, p<.01)$, meaning to high arousal $(\beta=.08, p<.01)$, and satisfaction to dampening savoring $(\beta=-.09, p<.01)$. Each non-significant path was removed sequentially from the least to most significant. At each stage of the pruning process, the model fluctuations in beta weights, phis, and error terms were assessed to ensure multicollinearity was not a significant issue. A total of 28 cross-lag paths were removed including the path from pleasure to satisfaction which became non-significant at the seventeenth step of pruning. The model fit after pruning slightly improved the $\chi^{2} / \mathrm{df}$ ratio to 2.74 , while all other indices remained identical to the saturated model. The final model included 17 significant crosslag paths and all 8 significant stability paths, as seen in Figure 5.4. For easier reading, significant paths, their standardised regression coefficient, and level of significance for the pruned path model are also represented in Table 5.5.

Table 5.5. Standardised Regression Coefficients for the Savoring, Hedonic, Eudaimonic, Satisfaction with Life, and Everyday Positive Life Events Pruned Structural Path Model.

\begin{tabular}{lcccccccc}
\hline & Damp2 & LA2 & HA2 & SF2 & Pleas2 & Mean2 & SWL2 & EPLE2 \\
\hline Damp1 & $.56^{* * *}$ & - & - & - & - & - & $-.04^{*}$ & $-.10^{* *}$ \\
LA1 & - & $.55^{* * *}$ & - & - & - & $.04^{*}$ & - & - \\
HA1 & - & - & $.76^{* * *}$ & - & - & - & - & $-.17 \dagger$ \\
SF1 & - & - & $-.42^{* * *}$ & $.61^{* * *}$ & - & - & - & $.21^{*}$ \\
Pleas1 & - & $.10^{* *}$ & $.10^{* *}$ & $.12^{* * *}$ & $.86^{* * *}$ & $-.09^{* * *}$ & - & $.15^{* * *}$ \\
Mean1 & - & $.08^{* *}$ & $.11^{* *}$ & - & $-.06^{* *}$ & $.89^{* * *}$ & - & - \\
SWL1 & $-.06^{*}$ & - & - & - & $.05^{*}$ & $.04^{*}$ & $.86^{* * *}$ & - \\
EPLE1 & - & - & - & - & - & - & - & $.45^{* * *}$ \\
\hline
\end{tabular}

Note. Damp = Dampening, LA = Low Arousal, HA = High Arousal, SF = Self-focus, Pleas = Orientation to Happiness Pleasure, Mean = Orientation to Happiness Meaning, SWL = Satisfaction with Life, and EPLE = Everyday Positive Life Events. Measures down the left side of the table are from Time 1. Measures across the top of the table are from Time 2, 3months after Time 1. ${ }^{* * *} p<.001 .{ }^{* *} p<.01{ }^{*} p<.05 .{ }^{\dagger} p=.07$. 
The finding that dampening savoring predicted a decrease in satisfaction and vice versa supported Hypothesis 8. Similarly, the finding that low arousal savoring manifested a bidirectional relationship with meaning orientation to happiness supports Hypothesis 8. Although high arousal savoring did not predict pleasure orientations to happiness, the finding that pleasure predicted high arousal savoring confirms Hypothesis 9. Contrary to Hypothesis 10, self-focus savoring and satisfaction with life were not directly related in the path model. Several general trends were revealed by the structural path model that had not been predicted. The path model indicates several directions of effect; satisfaction with life leading to orientations to happiness, orientations to happiness leading to savoring, and finally savoring leading to everyday positive life events (refer to Figure 5.4).

Bi-directional relationships. Three bi-directional relationships were revealed by the structural path model: dampening savoring and satisfaction, low arousal savoring and meaning, and pleasure and meaning orientations to happiness. Each of these bi-directional relationships was investigated in turn; first, within the pruned eight-variable path model (Figure 5.4), and second, in simplified and focussed two-variable path models. Equality constraints were applied to the bi-directional cross-lag paths to test for invariance. If the change in the chi square value and degrees of freedom between the baseline model (i.e. all paths freely estimated) and the constrained model (i.e. the two bi-directional paths constrained) was non-significant, then the relationship was considered invariant, (i.e. the bi-directional paths were of relative equal strength). On the other hand, if the change was significant, it would indicate that one variable had a stronger influence within the bi-directional relationship.

The chi square value and degrees of freedom for the baseline eight-variable path model $\left(\chi^{2}=2896.37, \mathrm{df}=1062\right)$ were significantly different to the model with equality constraints on the bi-directional paths between dampening savoring and satisfaction $\left(\chi^{2}=2901.13, \mathrm{df}=1061\right.$; $\left.\Delta \chi^{2}=4.75, \Delta \mathrm{df}=1, p<.05\right)$, indicating satisfaction inhibits dampening savoring $(\beta=-.06)$ significantly more than the reverse $(\beta=-.04)$. The chi square value and degrees of freedom for the baseline eight-variable path model were not significantly different to the model with equality constraints on the bi-directional paths between low arousal savoring and meaning $\left(\chi^{2}=2898.05\right.$, $\left.\mathrm{df}=1062 ; \Delta \chi^{2}=1.67, \Delta \mathrm{df}=1, p=.20\right)$. Similarly the base model was not significantly different to the model with equality constraints on the pleasure and meaning bi-directional relationship $\left(\chi^{2}\right.$ $\left.=2897.80, \mathrm{df}=1062 ; \Delta \chi^{2}=1.43, \Delta \mathrm{df}=1, p=.23\right)$. It appears that low arousal and meaning have an equally positive impact on one another, while pleasure and meaning orientations to 
happiness also have an equally negative impact on one another. It is worth noting that the measurement models at Time 1 and Time 2 evidenced moderate, positive, and highly significant concurrent correlations between pleasure and meaning; however, the present bi-directional model indicates pleasure orientations decrease meaning orientations over time and vice versa.

Focussed bi-directional path models. Next, each path model was reduced to only the two variables of interest modelled over time (i.e., a total of 4 latent variables) for each of the bidirectional relationships found in the eight-variable path model (Figure 5.4). These saturated models were tested for adequate model fit and significant regression coefficients. Constructing simplified models was a robust test of whether the bi-directional relationships were consistent without the influence of the larger path model.

The bi-directional model fit for dampening savoring and satisfaction with life was good $\left(\chi^{2} / \mathrm{df}\right.$ ratio $\left.=3.03, \mathrm{CFI}=.99, \mathrm{RMSEA}=.03, \mathrm{sRMR}=.03\right)$. The path from dampening savoring to satisfaction with life was weakly negative and significant, which was similar to the path from satisfaction to dampening savoring (Model A, Figure 5.5, p. 134). The difference in the chi square value and degrees of freedom between the base model $\left(\chi^{2}=127.37, \mathrm{df}=42\right)$ and the equality constraints model $\left(\chi^{2}=131.24, \mathrm{df}=43\right)$ indicated a marginally significant difference $\left(\Delta \chi^{2}=3.87, \Delta \mathrm{df}=1, p=.05\right)$. This finding replicates the outcome of the equality test within the larger path model that satisfaction's inhibition of dampening savoring is stronger than the negative impact of dampening savoring on satisfaction.

The bi-directional model fit for low arousal savoring and meaning orientation to happiness was good $\left(\chi^{2} / \mathrm{df}\right.$ ratio $\left.=1.98, \mathrm{CFI}=1.00, \mathrm{RMSEA}=.02, \mathrm{sRMR}=.03\right)$. The path from meaning to low arousal savoring was weak, but highly significant, however, the path from low arousal savoring to meaning was non-significant $(p=.23$ ) and was removed (Model B, Figure 5.5.). After pruning, the chi square improved slightly $\left(\chi^{2} / \mathrm{df}\right.$ ratio $\left.=1.97\right)$ and all other indices remained the same. In this isolated bi-directional model between low arousal savoring and meaning orientation, it was found that meaning positively influenced low arousal savoring strategies but that their relationship was not reciprocal, contrary to the findings within the complex structural path model in Figure 5.4.

Lastly, the bi-directional model fit for pleasure and meaning orientations to happiness was adequate except for the $\chi^{2} / \mathrm{df}$ ratio $\left(\chi^{2} / \mathrm{df}\right.$ ratio $=8.34, \mathrm{CFI}=.98, \mathrm{RMSEA}=.06$, sRMR $\left.=.06\right)$. The path from pleasure to meaning was weakly negative and highly significant, while the path from meaning to pleasure was also weakly negative, but marginally significant (Model C, Figure 5.5). The difference in the chi square value and degrees of freedom between the base model $\left(\chi^{2}\right.$ 
$=350.43, \mathrm{df}=42)$ and the equality constraints model $\left(\chi^{2}=352.31, \mathrm{df}=43\right)$ indicated a nonsignificant difference between the paths $\left(\Delta \chi^{2}=1.88, \Delta \mathrm{df}=1, p=.17\right)$, supporting an equal negative bi-directional influence between pleasure and meaning orientations to happiness across three months, consistent with the equality tests within the larger path model.

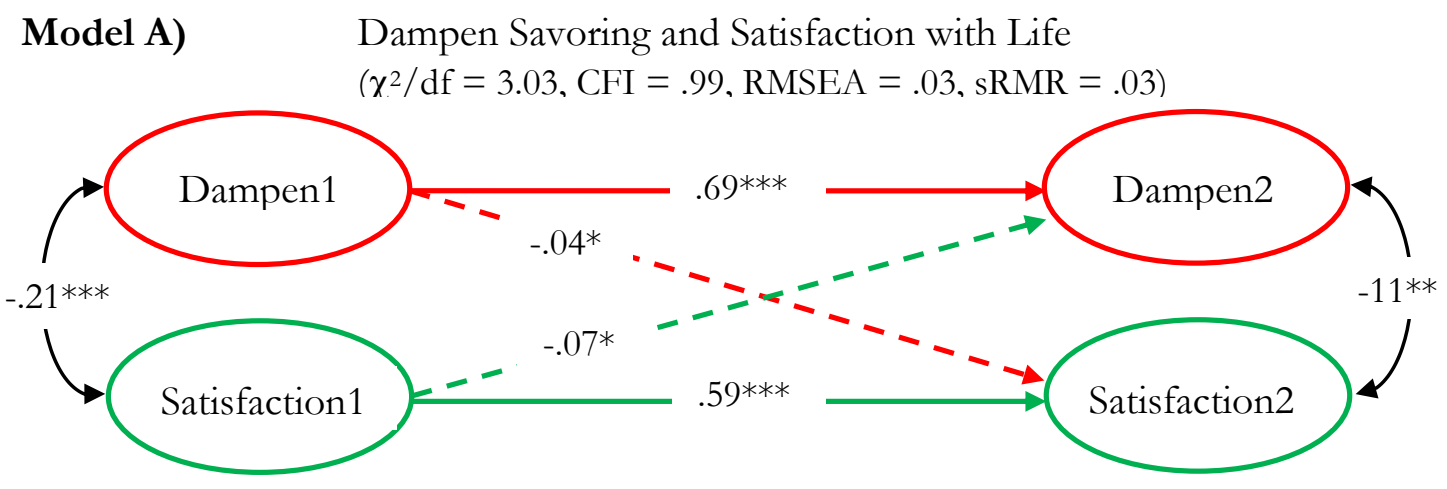

Model B) Low Arousal Savoring and Meaning Orientation $\left(\chi^{2} / \mathrm{df}=1.97, \mathrm{CFI}=1.00, \mathrm{RMSEA}=.02, \mathrm{sRMR}=.03\right)$
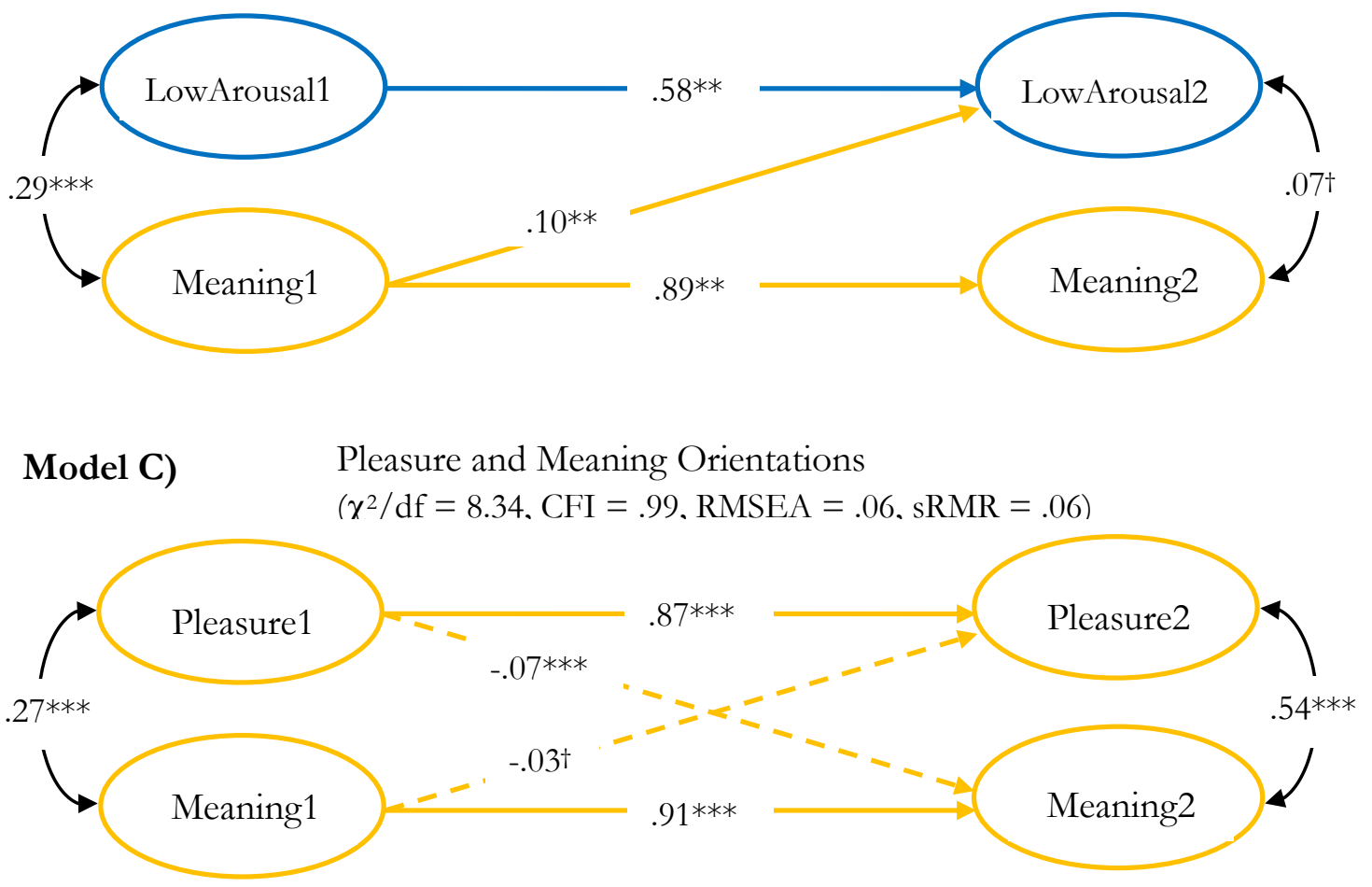

Figure 5.5. Final bi-directional models. Note. ${ }^{* * *} p<.001 .{ }^{* *} p<.01 .{ }^{*} p<.05 .{ }^{\dagger} p=.07$. 


\section{The moderating influence of pleasure and meaning orientations to happiness on savoring.}

The structural eight-variable path model revealed pleasure and meaning orientations were highly stable across three months, supporting their capability as possible moderators of savoring and positive events. Chapter 3 investigated the ability of savoring to moderate the relationship from hedonic wellbeing to eudaimonic wellbeing at one point in time. Chapter 4 found that everyday positive life events was not a predictor of savoring a month later, and this chapter found savoring predicted everyday positive life events three months later. This section turns to moderation analysis to expand on previous chapters and continue investigating savoring, positive events, and wellbeing.

To test the last two hypotheses, the relationship from savoring at Time 1 to everyday positive life events at Time 2 , moderated by average pleasure and meaning orientations was examined. Hypotheses 12 and 13 assumed orientations to happiness would buffer the impact of dampening on everyday positive events, and enhance the impact of low arousal, high arousal, and self-focus savoring on positive life events.

A total of 8 moderations were conducted in SPSS to observe whether average pleasure and/or meaning orientations moderated the relationship between each savoring strategy at baseline to positive events at 3-months. In other words, it was tested whether the relationship from dampening (or low arousal, high arousal, and self-focus) savoring at baseline to everyday positive events three months later was moderated by the average level of pleasure (meaning) across three months. All predictor variables were centred before creating interaction terms to reduce multicollinearity (Howell, 2009). The dependent variable, everyday positive life events at Time 2, was residualised by entering everyday positive life events at Time 1 in Step 1 . The main effects of the predictor and moderator were entered in Step 2 (e.g. high arousal at Time 1 and average meaning). The interaction between the main effect and moderator was entered in Step 3 (e.g. high arousal at Time $1 \mathrm{x}$ average meaning).

In total, four significant moderations were found, all with meaning orientation moderating the relationship from savoring to positive life events. Table 5.6 provides the regression results for all four significant moderations. Meaning buffered the negative impact of

Table 5.6. The Impact of Savoring on Positive Events Moderated by Meaning Orientation. 
Intensity Everyday Positive Life Events Time 2

\begin{tabular}{|c|c|c|c|c|c|}
\hline Variable & $B$ & $S E B$ & $\beta$ & $\Delta R^{2}$ & $\operatorname{Sign} F \Delta$ \\
\hline Step 1 & & & & .21 & $<.001$ \\
\hline PosEvents T1 & .46 & .02 & $.46^{* * *}$ & & \\
\hline Step 2 & & & & .01 & $<.001$ \\
\hline $\begin{array}{l}\text { Dampening T1 } \\
\text { Average Meaning }\end{array}$ & $\begin{array}{r}-.01 \\
.02\end{array}$ & $\begin{array}{l}.02 \\
.004\end{array}$ & $\begin{array}{l}-.02^{\text {ns }} \\
.11^{* * *}\end{array}$ & & \\
\hline Step 3 DampxMean & .01 & .003 & $.05^{*}$ & .002 & .026 \\
\hline
\end{tabular}

Intensity Everyday Positive Life Events Time 2

\begin{tabular}{|c|c|c|c|c|c|}
\hline Variable & $B$ & SE B & $\beta$ & $\Delta R^{2}$ & Sign F $\Delta$ \\
\hline Step 2 & & & & .01 & $<.001$ \\
\hline Low Arousal T1 & .01 & .02 & .02 & & \\
\hline Average Meaning & .02 & .004 & $.11^{* * *}$ & & \\
\hline $\begin{array}{l}\text { Step } 3 \text { LAxMean } \\
\quad\end{array}$ & .006 & .003 & $.06^{*}$ & .003 & .056 \\
\hline
\end{tabular}

Intensity Everyday Positive Life Events Time 2

\begin{tabular}{lccccc}
\hline Variable & $B$ & SE B & $\beta$ & $\Delta R^{2}$ & Sign F \\
\hline Step 2 & & & & .01 & $<.001$ \\
$\quad$ High Arousal T1 & .04 & .02 & $.05^{*}$ & & \\
$\quad$ Average Meaning & .02 & .004 & $.11^{* * *}$ & & \\
\hline Step 3 & & & & .002 & .041 \\
$\quad$ HAxMean & .006 & .003 & $.04 *$ & & \\
\hline
\end{tabular}

Intensity Everyday Positive Life Events Time 2

\begin{tabular}{|c|c|c|c|c|c|}
\hline Variable & $B$ & SE B & $\beta$ & $\Delta R^{2}$ & Sign FA \\
\hline Step 2 & & & & .01 & .001 \\
\hline Self-Focus T1 & .04 & .02 & $.05^{*}$ & & \\
\hline Average Meaning & .02 & .004 & $.11 * * *$ & & \\
\hline $\begin{array}{ll}\text { Step } 3 & \\
& \text { SFxMean }\end{array}$ & .008 & .003 & $.06^{* *}$ & .003 & .005 \\
\hline
\end{tabular}

Note. Step 1 in the first regression analysis was identical for each subsequent regression analysis. PosEvents \& $\mathrm{PE}=$ Everyday Positive Events, T1 = Time 1, Damp = Dampening, LA = Low Arousal, HA=High Arousal, $\mathrm{SF}=$ Self-focus. ${ }^{* * *} p<.001,{ }^{* *} ; p<.01 ;{ }^{*} p<.05 ;{ }^{\dagger} p<.10,>.05$.

dampening on positive events, as predicted by Hypothesis 13. A simple slope analysis revealed that the slope of both high $(.02, s e=.02, p=.35)$ and medium levels of meaning $(-.01, s e=.02, p$ $=.35$ ) were not significantly different from zero. The slope of low meaning, however, was significantly different from zero $(-.05, s e=.02, p<.05)$, indicating that while high and medium levels of meaning may help withstand the adverse impact of dampening on positive events (i.e. buffer it), the relative absence of meaning allows the negative association between dampening strategies and the intensity of positive events to become evident (see Model A, Figure 5.6). 

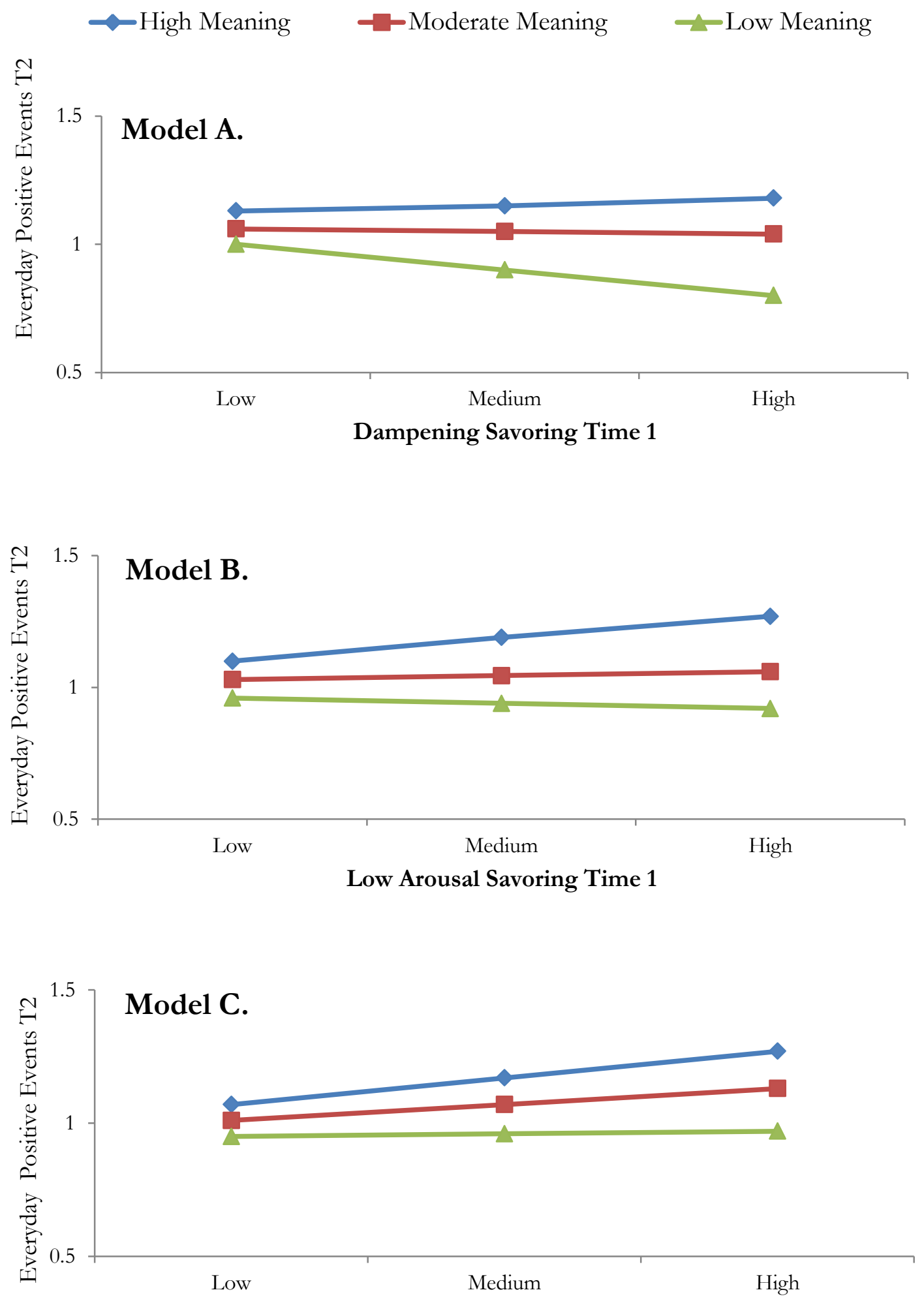

High Arousal/Self-focused Savoring Time 1

Figure 5.6. Savoring at Time 1 and everyday positive life events at Time 2 moderated by average meaning orientation to happiness. 
As predicted, meaning enhanced the positive impact of low arousal, high arousal, and self-focused savoring on everyday positive life events (Hypothesis 13). Meaning had a slightly different impact on low arousal savoring and positive events than it did on high arousal savoring and positive events and self-focus savoring and positive events. For low arousal savoring and positive events, only the slope of high meaning was significantly above zero $(.05, s e=.02, p<$ .05). High levels of meaning enhanced the relationship from low arousal savoring to positive events, whereas moderate and low levels of meaning yielded essentially flat relationships between low arousal savoring and positive events (see Model B, Figure 5.6).

Moderate and high levels of meaning both enhanced the relationship from high arousal and self-focus savoring to positive life events. The slope for moderate $(.05 s e=.02, p<.05)$ and high levels of meaning $(.08, s e=.03, p<.01)$ were above zero for the relationship from high arousal savoring to positive events. Similarly, the slope for moderate $(.04 s e=.02, p<.05)$ and high levels of meaning $(.09, s e=.02, p<.001)$ were above zero for the relationship from selffocused savoring to positive events (see Model C, Figure 5.6). These findings show that while low levels of meaning had no enhancing impact, holding moderate or high meaning levels did. Further, high levels of meaning had the steepest slope, indicating that the greatest amplification of the relationship between high arousal/self-focused savoring and positive events was from high meaning, although moderate meaning still had a significant positive impact. Pleasure (i.e. hedonia), however, was unable to buffer or enhance the relationship from savoring to everyday positive events, contrary to Hypotheses 12 and 13.

\section{Discussion}

This chapter examined the psychometric properties of the abridged WOSC, as well as the differences and directions of effect among adults' savoring strategies, components of wellbeing, and positive life events over three months. Four broad research questions guided the current chapter: (1) Are the four factors of adult savoring consistent across time, stable over time, and relatively independent as was found with adolescents? (2) Do adults at different stages of adulthood differ in their savoring strategies, orientations to pleasure and meaning, satisfaction with life, and everyday positive events? (3) What are the directions of effect among savoring strategies, wellbeing indicators, and everyday positive life events? and (4) Do orientations to happiness moderate savoring relationships? In addition, based on the interesting findings under research question 3 , exploratory analyses of bi-directional relationships were conducted. The research questions, their related hypotheses, and exploratory findings are discussed in turn below. 


\section{Hypotheses 1 through 4: Psychometric tests of savoring}

The first, third, and fourth hypotheses were supported, while the second hypothesis was partially supported. First, the four savoring factors were confirmed with nearly 2,000 international adults at baseline and three months later using confirmatory factor analysis, and the savoring factor loadings, factor variances, and factor covariances were all invariant across the three months. The savoring test-retest reliability was moderate to high and each factor predicted itself across three months over and above predicting any other savoring factor, supporting Hypotheses 3 and 4. The four-factor savoring construct was psychometrically robust under stringent tests of its factor structure with adults across three months, similar to previous findings with adolescents across four weeks. Although the adolescent and adult samples were different in multiple ways, the savoring measure was consistently reliable and stable across time from four weeks through three months. Importantly, the strength of the abridged measure to perform under such rigorous analyses with varied demographics encourages its generalisation and continued reliable use.

Age did not evidence as strong an influence on covariances involving dampening savoring as was found between adolescents and adults in Chapter 2, partially supporting Hypothesis 2 . The correlation between dampening and low arousal savoring significantly differed between young and middle adults at both baseline and three months, but no other differences in dampening covariances were found. It appears young adults differentiate their dampening savoring from high arousal and self-focus savoring to the same degree as middle and older adults, and that young and older adults have a similarly strong association between their dampening and low arousal savoring. This association between dampening and low arousal strategies across adulthood is slightly ' $U$ ' shaped, where young adults showed the highest association, middle adults the lowest, and older adults somewhere in between-not significantly different from either young or middle adults. These findings implicated a trend where the ability to differentiate savoring abilities, particularly dampening from low arousal strategies, was still being formed in young adulthood. This study is the first to specify where potential developmental influences would be most impactful on savoring, and established a focussed target for further study and building individual savoring abilities.

\section{Hypotheses 5 through 7: Developmental differences in savoring, wellbeing, and positive events}

Hypotheses 5 through 7 were generally well supported. Younger adults and middle aged adults were both significantly higher on dampening strategies than older adults. Although 
younger adults reported more dampening strategies than middle adults, the difference was not significant. No differences in low arousal savoring across adulthood were found, supporting Hypothesis 6 and in line with research showing that low arousal emotions are consistent across the life span (Pinquart, 2001). As expected, however, high arousal savoring decreased with age. With each increase in age, from young to middle to older age, high arousal savoring significantly diminished, which supports previous research on emotions (Pinquart, 2001). The last savoring strategy, self-focus savoring, showed an inverted ' $U$ ' shape: in young and older adulthood selffocus savoring was lower than in middle adulthood. Perhaps this spike in self-focus savoring reflected engaging in more frequent emotional experiences of gratitude and pride during mid-life.

Young adults were more pleasure oriented than both middle and older adults, as hypothesised. Although middle adults reported more pleasure orientation than older adults, the difference was non-significant. On the other hand, older adults were more meaning oriented than both young and middle adults, and middle adults were marginally more meaning oriented than younger adults. It is noteworthy that all age groups reported a stronger orientation toward meaning than pleasure, although no tests of significance were conducted (refer to Table 5.3 for means and standard deviations of all measures). Together these findings indicated a linear trend across adulthood where the desire for pleasure wanes, while the desire for meaning waxes, and there is a tendency to endorse meaning over pleasure throughout adulthood.

In support of the fifth hypothesis, the intensity of positive events linearly decreased across each of the three age groups. This finding extends previous work by Charles et al. (2010) that found 101 women 63 - 93 years old reported a decrease in the intensity of positive events with age. The present study supported this developmental trend with a substantially larger and more diverse sample of males and females. Arguably, as an individual ages, the variety and quantity of events they have experienced becomes ever greater. The cumulative exposure to positive events with age makes positive events ordinary, rather than novel and deserving of curiosity. The intensity wears off. In other words, it appears individuals become increasingly habituated to positive events with age.

\section{Hypotheses 8 through 11: The direction of effect between savoring, wellbeing, and positive events}

Hypotheses 8 through 10 were supported, but Hypothesis 11 was not. In addition, several relationships that were not hypothesised became evident after viewing the pruned initial direction-of-effect path model. Before discussing the hypotheses it is worth discussing the stability coefficients. Pleasure and meaning orientations and satisfaction with life were highly 
stable across three months: baseline pleasure accounted for $74 \%$ of the variance in pleasure at 3months, baseline meaning accounted for $79 \%$ of the variance in meaning at 3 -months, and baseline satisfaction accounted for $74 \%$ of the variance in satisfaction at 3-months. As expected, happiness orientation and satisfaction appeared more trait-like than state-like. Although little variance was left to account for in these constructs, they were still predicted by factors at Time 1 other than themselves. On the other hand, the average savoring strategy at baseline predicted $32 \%$ of its variance at 3-months, and baseline positive life events predicted the least variance at 3-months, 20\%. Overall these findings indicate that orientations to happiness and satisfaction with life are the most stable, savoring is moderately stable, and everyday positive life events are the least stable over three months. Although there were no hypotheses regarding stability, these findings are not unexpected and are congruent with theory and previous evidence (Bryant \& Veroff, 2007; Diener, Scollon, et al., 2009).

Returning to the hypotheses, as predicted, dampening was negatively associated with satisfaction with life. Dampening both predicted and was predicted by satisfaction, and it reduced the intensity of positive events over three months. Dampening savoring at baseline reduced individuals' positive evaluations of their life $(.2 \%)$ and the intensity of their positive events $(1 \%)$ at 3 months. In turn, holding positive evaluations of one's life decreased (.4\%) thinking that one is undeserving of good events or that an event is not as good as it could be ( i.e. dampening savoring). The negative impact of dampening on everyday positive events was not hypothesised — savoring theory indicates the direction of effect is generally from events to savoring - it is, however, reasonable. Less discussion has existed for the role of savoring as a predictor of positive events, yet the findings here indicate it deserves further attention and theory development.

Low arousal savoring was positively associated with meaning orientation, as hypothesised. It both predicted and was predicted by meaning orientation to happiness over three months. Although the only variable low arousal predicted was meaning, both meaning and pleasure predicted low arousal savoring. It appears that absorbing the moment and focussing on particular senses is good for increasing meaning, and both meaning and pleasure are partially responsible for increases in low arousal strategies.

High arousal savoring was positively associated with pleasure orientation as hypothesised. However, only pleasure predicted high arousal, high arousal did not predict pleasure. Meaning also positively predicted high arousal, which was not hypothesised, and high arousal negatively predicted everyday positive life events, which was not hypothesised. Those adults who had a 
disposition toward meaning or pleasure savored by looking for people to share their positive experience with, and laughed or giggled (i.e. high arousal savoring). On the other hand, using these high arousal savoring strategies diminished the intensity of positive events over time. Interestingly, high arousal savoring and positive events were strongly associated at one point in time; however, this savoring strategy seemed to diminish adults' intensity of positive experiences over time.

Contrary to the last hypothesis, self-focus savoring at baseline was not related to satisfaction with life at 3 months. Self-focus savoring was expected to be positively related to satisfaction as was found with concurrent data in Chapter 3, but apparently this relationship was unable to survive over three months, or perhaps it was overshadowed by stronger competing relationships in the complex path model. Self-focus savoring did, however, positively predict positive events, and was positively predicted by pleasure orientation. Further, out of the three savoring strategies that predicted positive events, self-focus savoring at baseline was responsible for the greatest variance in the intensity of positive events at 3 months, $4 \%$. Counting blessings, congratulating oneself, and constructing memories for later recall increased the intensity of positive events over time. Lastly, holding a pleasure orientation to happiness at baseline, not a meaning orientation, increased self-focus savoring three months later.

Comparing the relationships with high arousal and self-focus strategies, it was found that self-focus predicted increases in positive events, while high arousal strategies predicted decreases. It is possible that high arousal strategies interfered with the intensity of positive events over time as they tend to involve high levels of energy creating and enhancing intensive emotions (e.g. excitement in the moment). One could speculate that this process would lead to rapid fall-off (quick habituation and fatigue), whereby recognising or experiencing the impact of subsequent positive events was diminished. On the other hand, more contemplative strategies (i.e. self-focus savoring) tend to expend less emotional energy (e.g. cultivating pride and gratitude), and they seem to enhance emotions that are future-directed and recognise and cultivate the impact of positive events.

Returning to the relationships between savoring and orientations, previous research speculated that the pursuit of meaning had a stronger influence on wellbeing than pleasure (Schueller \& Seligman, 2010). When considering savoring, however, pleasure orientations positively predicted all three amplifying savoring strategies, while meaning predicted two, and pleasure seemingly had a stronger positive relationship with savoring than an orientation toward meaning (although no statistical test of difference was conducted). The repeated strong links 
between pleasure and savoring were consistent with a central tenet of savoring theory that states that savoring principally occurs within the context of experiencing positive emotions. Although the exploration of the intricacies between savoring and eudaimonia was warranted, the theoretical guidance for this examination, at this juncture, is underdeveloped (Bryant, et al., 2011; Bryant \& Veroff, 2007). In sum, having an orientation toward pleasure yielded stronger associations and more associations over time with savoring than an orientation toward meaning; however, meaning orientation may indeed have a stronger influence on wellbeing indicators as previously speculated, a topic that is discussed below in relation to satisfaction with life.

Other findings from the direction-of-effect path model that were not hypothesised but were mentioned for exploration included: the relationship between pleasure, meaning, and satisfaction; the direction of effect between savoring and positive life events; and bi-directional relationships. I will review each of these in turn.

Pleasure, meaning, and satisfaction. Although previous research and the present study found that orientations toward pleasure and meaning were highly and positively related concurrently (Park, et al., 2009; Peterson, et al., 2005; Peterson, et al., 2007; Ruch, et al., 2011; Vella-Brodrick, et al., 2009), pleasure interfered with meaning and meaning interfered with pleasure over time. The findings questioned the positive predictive relationship from pleasure (hedonia) to meaning (eudaimonia) that is often conceptualised in the literature (e.g. Fredrickson, 2002). Although the negative relationship between hedonia and eudaimonia is not difficult to imagine and often debated, it has rarely been empirically regarded (Huta \& Ryan, 2010).

Consider Steve, a caricature, as an example of the potential interference between the pursuit of pleasure and the pursuit of meaning. Steve had a highly pleasurable time with friends at a Saturday night party that spilled into the early morning hours. On Sunday morning Steve usually goes to church with his family, but his night of fun made him incapable this time. As a consequence Steve missed the sense of purpose he usually gained by attending church—his pleasurable experience cost him his meaningful experience the day after. In the reverse, imagine a Steve that held puritanical beliefs that consistently interfered with his ability to embrace joy and happiness.

Granted, this example with Steve is caricature, but the interference between pleasure and meaning can be imagined in more subtle and frequent situations as well. The pleasure gained from consumerism, for instance, can be interrupted by the desire for purpose and meaning. If an individual was feeling pleasant while consuming and was interrupted by meaning, which made them consider the ethical impact of their purchases (e.g., the stripping of natural resources and 
the exploitation of labour), it is likely to challenge them, possibly engender guilt, and in turn have detracted from their pleasure. As the results indicated, recall, the interference goes both ways. The findings pointed out that too much happiness in one domain will interfere with happiness in another domain—balance is important for a full life (for a discussion on balancing happiness, see Sirgy \& Wu, 2009).

This study next clarified the direction of effect between orientations and satisfaction. Although previous research conceptualised orientations as predicting satisfaction (e.g. VellaBrodrick, et al., 2009), the results indicated satisfaction led to orientations. The correspondence between orientations and satisfaction was uni-directional, from baseline satisfaction to pleasure and meaning at 3 months. Satisfaction with life increased orientations toward both a pleasurable and meaningful life, not the other way around. As discussed above, previous research (Schueller \& Seligman, 2010) speculated that meaning orientation had a stronger influence on wellbeing than a pleasure orientation. In contrast, the present study indicated that satisfaction more strongly predicted pleasure orientation than meaning orientation, although a statistical test of difference was not conducted. With this evidence, future research is encouraged to expand their investigation of orientations to include how and why satisfaction leads to hedonic and eudaimonic dispositions over time, and explore whether meaning is more highly predictive of or predicted by satisfaction than pleasure. This study is among one of the first to clarify the directional influence between satisfaction and orientations, and it showed that dispositional life satisfaction led to the pursuit of pleasant and meaningful living.

Savoring and positive events. The direction of effect between positive events and savoring were contrary to the general conceptualisation - that savoring results from positive events. Over three months, the intensity of everyday positive events failed to predict anything in the model, however, three of the four savoring strategies predicted positive events. Self-focus savoring increased the intensity of positive events, while dampening and high arousal savoring decreased it, indicating that how an individual thinks and behaves about a pleasant event at one point-in-time influenced whether they felt more or less moved by their positive events later. There may, however, be a causal relationship from positive events to savoring that occurs under shorter durations of time (Bryant, et al., 2011), for example, with daily events (Jose, et al., 2011). Taken as a whole, the direction of effects, excluding bi-directional relationships, were from satisfaction with life to happiness orientations to savoring and finally to positive events. Several bi-directional relationships were evident within the large residualised path model. To investigate these further, follow-up exploratory analyses were conducted. 


\section{Exploratory analyses and findings: $\mathrm{Bi}$-directional relationships}

Three bi-directional relationships were identified within the larger residualised path model: dampening and satisfaction, low arousal savoring and orientation toward meaning, and orientations toward pleasure and meaning. Satisfaction diminished dampening savoring slightly more than dampening savoring diminished satisfaction. With more satisfaction, individuals increasingly used less dampening savoring, although dampening inhibited satisfaction to a lesser degree. In the context of a path model involving several variables, low arousal savoring enhanced meaning, however, when low arousal savoring was simply paired with only meaning it had no influence on meaning orientation. Meaning, however, still promoted strategies for absorbing the moment and sensory perceptual sharpening.

Expanding the previous discussion, orientations toward pleasure and meaning had an equally negative effect on each other over the three months, which suggested the two domains of happiness exist in equilibrium with each other over time. It is possible that individuals who attempt to simultaneously live with both high pleasure and high meaning find this difficult to do in the long term, since pleasure and meaning seem to attenuate one another over time. If this is true, individuals might be encouraged to compartmentalise or focus on living the pleasurable and meaningful life at discrete periods of time, rather than aiming to engage in both pursuits at the same time.

\section{Hypotheses 12 and 13: The moderating influence of pleasure and meaning orientations to happiness on savoring}

Orientations to happiness were expected to buffer the negative impact of dampening on everyday positive events (Hypothesis 12), and enhance the positive impact of low arousal, high arousal, and self-focus savoring on everyday positive life events (Hypothesis 13). Both expectations were supported, but only for orientation toward meaning, as orientation toward pleasure proved not to be a significant moderator.

Consider how a high orientation toward meaning compared to a low orientation toward meaning can buffer dampening and enhance amplifying savoring. Take Erin, an individual with a high orientation toward meaning, and Sam, an individual with a low orientation toward meaning. Although Erin and Sam both reported dampening, Erin's inclination to derive purpose and meaning buffered the effects of dampening on her intensity of positive events, however, Sam lacked the inclination for meaning, so her dampening strategies went unabated and reduced the intensity of her subsequent positive events. Say for instance that Erin and Sam both received a letter from a good friend, and both thought about how it was not as open and sharing as they 
had hoped (i.e. dampening). For Erin, her ability to see the purpose of her friend's letter prevented her dampening strategies from ruining the intensity of subsequent positive events. In contrast, Sam's lack of desire for meaning sustained her sour interpretation of her letter and marginalised the intensity of her subsequent positive events. In a similar vein, although Erin and Sam both reported self-focused savoring, Erin's ability to find purpose and have a sense of her place in society enhanced her savoring and thus the intensity of her subsequent positive events. Sam, on the other hand, was unable to understand her experience as having a wider implication and therefore her savoring was not as powerful. Obvious from these examples, having a high orientation toward meaning was an important dispositional factor for turning down the volume on dampening, and turning up the volume on the positive impact of amplifying strategies on subsequent positive events.

This final empirical chapter was able to psychometrically support the four-factor savoring structure to a high degree with adults over three months. In addition, the longitudinal analyses evidenced a trend in the direction of effect from trait to state constructs, in other words, from satisfaction with life to happiness orientations to savoring strategies, and finally, to positive events. Several relationships were highlighted that questioned previous assumptions, for instance, that satisfaction with life leads to orientations toward pleasure and meaning, and that positive events lead to savoring. Again there were age differences for savoring, however, they were less pronounced than found in previous chapters, indicating changes across adulthood are perhaps more subtle than from adolescence to adulthood. The contributions, implications, and suggestions for future study from this thesis are discussed in relation to theories of savoring and wellbeing in the final chapter that follows. 


\section{CHAPTER 6}

\section{Contributing to Savoring and Wellbeing Research}

We advocate learning how to cultivate savoring... With that capacity, people can better enjoy love, truth, beauty, community, God, sexuality, spirituality, or whatever preferred values and individual goals they deem important.

(Bryant \& Veroff, 2007, p. xi)

The present research aimed to understand adolescent and adult savoring, its involvement with everyday positive life events, and hedonic and eudaimonic wellbeing. The research was designed with principles explicit to savoring theory (Bryant \& Veroff, 2007) and the broaden-and-build theory (Fredrickson, 1998), and was accomplished with concurrent and longitudinal quantitative research methods. I first used a theoretical and empirical approach (Koole, 2009) to analyse a dataset drawn from an abridged form of the Ways of Savoring Checklist to investigate the similarities and differences between adolescent and adult savoring, and the findings were presented in Chapter 2. I then examined the similarities and differences between adolescent and adult concurrent relationships for savoring and subjective wellbeing, and this information was presented in Chapter 3. This chapter was followed by focused analyses of adolescent savoring and wellbeing over time, see Chapter 4, and adult savoring and wellbeing over time, see Chapter 5.

The present, final, chapter provides a critical discussion of the contribution of this thesis to theory and practice with an awareness that the study of positive cognitive-behavioural processes is in its infancy, especially when considering developmental influences. I begin with a discussion of the findings, followed by a discussion of the strengths and implications of the present research. Next, limitations and suggested future directions of this research are detailed. Lastly, I present the conclusions to the present research.

\section{Key Findings}

The findings collectively supported four types of everyday savoring strategies that were found to be consistent across age (i.e. adolescence and adulthood), time, and gender. Further, robust associations between savoring and wellbeing indicated that savoring played an important role in both feeling good (i.e. hedonia) and functioning well (i.e. eudaimonia) for adolescents and adults. Although many relationships were comparable between adolescents and adults, several potential developmental influences on savoring and wellbeing were important to consider.

The findings provided unique and fertile contributions to the understanding of savoring and wellbeing, and implicated paths for increasing particular types of wellbeing as well as general wellbeing. The following section integrates the key findings for adolescents and adults, first by 
discussing the structure of savoring and their similarities and differences across age, second, the relationship between everyday positive events and savoring, and third, the relationship between savoring and wellbeing.

\section{Similarities and differences amongst savoring strategies across age}

In general there were more similarities than differences between adolescent and adult savoring (Chapter 2), and between young adult, middle adult, and older adult savoring (Chapter 5). A consistent four factor structure of savoring emerged, which included dampening, low arousal, high arousal, and self-focus savoring. Stringent testing returned strong psychometric support for this structure across four age groups, over several weeks (4 weeks for adolescents, and twelve weeks for adults), and across gender for adolescents.

A key difference was found whereby the positive association between dampening savoring and low arousal savoring gradually lost strength with age, from adolescence (13 to 15 years) to young adulthood (15 to 30 years) to middle adulthood (31 to 49 years) (Figure 2.2; Figure 5.2). In addition, adults appeared to report stronger differences for how much they used each savoring strategy compared with adolescents (Table 3.1). When observing the differences between age groups in reported dampening, adolescents favoured more dampening strategies than adults, while adults favoured more amplifying strategies (i.e. high arousal and self-focus) (Chapter 2). Within the adult study (Chapter 5), the findings further supported a linear decrease in the use of dampening with age, from young adults, to middle adults, and last older adults (Figure 5.3). However, no linear increase in amplifying strategies was noted for this sample. Instead, young adults reported higher rates of high arousal savoring than middle adults, who reported more than older adults. Adolescence appeared to be a discrete stage where dampening is highest, yet high arousal is lowest (refer to the Discussion section of Chapter 2 for a theoretical and empirical explanation of these findings).

Cumulatively the findings suggested that although savoring was arguably more similar than different across age, potential developmental effects contributed to the strength of particular savoring associations. These findings extended support for the theory that savoring begins to fully emerge in adolescence (Bryant \& Veroff, 2007). Earlier research showed that young children savor globally, but were unable to differentiate savoring in the past, present, or future (Cafasso, et al., 1994). Other research found savoring was differentiated in adolescence (Meehan, et al., 1993) and was fully formed in adulthood (Bryant, 2003). The findings here were in agreement with the extant literature that adolescents' and adults' savoring strategies shared more savoring similarities than differences. For the first time with specific savoring mechanisms, 
however, this research highlighted important potential differences in savoring development between adolescents and adults.

\section{Savoring and everyday positive events}

Everyday positive events were strongly associated with savoring in concurrent data (Chapter 4 and 5), and savoring increased the positive impact of events on concurrent positive emotions (i.e. full mediation), as savoring theory predicted (Chapter 4). Over time, however, events were neither a predictor of adolescent nor adult savoring. In fact, the reverse was found to be true in that each savoring strategy predicted changes in the intensity of positive events over three months for adults (Figure 5.4). This finding suggests adding specificity to a central tenet of savoring theory — that savoring enhances positive events—by speculating a time frame for the relationship from events to savoring.

Another possibility is that arguably the type of savoring investigated over time was indicative of trait more than state savoring, although the standardised stability coefficients (betas) were lower for savoring (.56 to .76) than for more trait-like measures (i.e. orientations to happiness; .86 and .89). Recall from the first chapter that savoring theory postulates that savoring strategies can reflect both stable personality traits as well as momentary state reactions to events (Bryant \& Veroff, 2007). The present study asked about savoring strategies used in relation to everyday positive events that occurred over the previous month (adolescents) and three months (adults), rather than momentary daily events (e.g. with experience sampling). Savoring theory, however, does not specify that dispositional (trait) savoring is a predictor of the intensity of positive events. This thesis provided potential specificity for the theory of savoring, indicating that dispositional savoring is a consistent predictor, not a criterion variable, of positive events over time.

\section{Savoring and subjective wellbeing}

Savoring was significantly and moderately to strongly related to wellbeing. The direction of this relationship was as expected. Also as expected, dampening savoring was negatively associated with wellbeing. In contrast, amplifying savoring was positively associated with wellbeing. Analyses from Chapters 3 and 4 found that self-focus savoring was the most useful strategy for adolescence. It was positively associated with the greatest number of wellbeing indicators (Chapter 3) and was the adolescents' only amplifying strategy to predict positive affect over time (Chapter 4). Past research on counting blessings (one of the specific self-focus strategies) and gratitude highlighted their importance in processes that promote adolescent wellbeing (Froh \& Bono, 2011; Froh, et al., in press; Froh, et al., 2008; Froh, et al., 2009). 
A combination of low arousal, high arousal, and self-focus savoring (i.e. amplifying) was important for a holistic increase in adult wellbeing (Chapter 3); for instance, only low arousal predicted eudaimonia, only high arousal predicted hedonia, and only self-focus savoring predicted satisfaction concurrently. Over time, adults' hedonia predicted all three types of amplifying savoring (i.e. low arousal, high arousal and self-focus savoring). In comparison, eudaimonia predicted two (i.e. low arousal and high arousal).

The findings from Chapter 3 showed that adolescent and adult savoring also moderated the relationship from hedonia to eudaimonia. Adolescent low arousal savoring moderated hedonia to eduaimonia, and adult low arousal and high arousal savoring were moderators. However, the two age groups' low arousal savoring moderated the hedonia - eudaimonia relationship differently. Adolescents with low levels of low arousal savoring manifested no better functioning (i.e. eudaimonia) than adolescents who reported high levels of low arousal savoring when their hedonia was high (Figure 3.4). In contrast, adults who reported high levels of high arousal savoring manifested significantly higher functioning than those adults who reported low levels of high arousal savoring when experiencing strong pleasant feelings (hedonia). Adult high arousal savoring moderated hedonia and eudaimonia similarly to that found for low arousal moderation.

Hedonic wellbeing. As expected, the final empirical chapter (Chapter 5) found that young adults pursued the pleasurable life more than middle or older adults. Previous research supported this finding with evidence that young adults defined happiness in more pleasure oriented terms, while older adults adopted more meaning oriented definitions (Kamvar, et al., 2009; Mogilner, et al., 2011).

Adolescents' positive affect fully mediated the relationship between positive events and eudaimonia, as well as from self-focused savoring to eudaimonia both concurrently and longitudinally (Figures 4.4 and 4.5). These findings supported the power of positive emotions to increase functioning and thus supported the broaden-and-build theory of positive emotions (Fredrickson, 1998).

Bryant and Veroff (2007) speculated that savoring was a mechanism within the broadening and building of positive emotions. They suggested that if positive emotions predicted savoring then savoring would be implicated as a mechanism for expanding an individual's cognitive and emotional repertoire. Fredrickson (2004) similarly hypothesised that contentment, love, and interest, for example, sparked increased attempts to savor. The findings here, however, were not supportive of the proposed role of savoring within the broaden-and-build model. 
When observing the bidirectional relationship between savoring and positive emotions, positive emotions did not predict savoring over time (Figure 4.2), contrary to expectations. The lack of a predictive relationship from positive emotions to savoring might in part be due the time frame under which the two were measured. Bryant and colleagues (2011) have recently suggested that to better understand the role of savoring within the broadening and building of positive emotions requires examination under short durations of time; for example, observing real-time savoring in naturalistic settings. The findings here, however, were able to support the important predictive role of savoring and its ability to initiate the broadening-and-building process through mediational analyses. Adolescents who counted their blessings, self-congratulated, and thought about remembering their positive events in the future experienced heightened positive emotions that in turn increased their social connectedness, autonomy, and purpose (i.e. eudaimonia). This finding provided empirical evidence for re-examining the theoretical role of, at least everyday, savoring in the broaden-and-build model of positive emotions. Taken together, although the findings here did not support the current specific theoretical role of savoring within the broadenand-build model, it did highlight the important impact of savoring on positive emotions and subsequent eudaimonia.

Eudaimonic wellbeing. When comparing age groups, older people were more meaning oriented than the younger and middle aged adults, and younger individuals were more pleasure oriented, as expected. This study, like studies before (Peterson, et al., 2005; Ruch, et al., 2011), provide evidence for the idea that younger adults seek a life of pleasure more so than older adults. However, an important finding from Chapter 5 deserved to be highlighted here. Meaning was universally more important than pleasure, from young to old; each age group pursued meaning more than pleasure.

From the fields of education and developmental psychology, Damon $(1995,2004,2008)$ has unfalteringly advocated for understanding adolescents and young adults as purposeful individuals who fundamentally desire meaning. Damon explained that in large part homes and schools have lacked recognizing this need in youth and have equally lacked the expectation of youth to pursue meaning. Future research can play a role in better understanding the drive for meaning in youth as well as the association between savoring and different types of meaning across adulthood (Steger, Frazier, \& Oishi, 2006). A description of these considerations is expanded under Future Directions below.

Similar to concurrent findings (Chapter 3), adult low arousal savoring was the only savoring strategy to predict meaning over time within a larger path model (Chapter 5). On its own, however, low arousal savoring did not affect meaning over time. It appears that in 
conjunction with other savoring strategies, low arousal savoring is important for boosting meaning, whereas meaning increases low arousal savoring regardless of its association with other variables.

In addition to meaning being an important predictor of low arousal savoring, meaning was a significant moderator of savoring. Adult meaning moderated the influence of all four savoring strategies on everyday positive events in the predicted direction (Figure 5.6).

Specifically, meaning buffered the impact of dampening savoring on everyday positive events and enhanced the impact of amplifying savoring (i.e. low arousal, high arousal, and self-focused savoring) on everyday positive events. For instance, dampening decreased the intensity of positive events over time, more for adults reporting low meaning, than for adults reporting high meaning. Further, those adults with high levels of meaning reported no significant decreases in the intensity of their events regardless of if they dampened a little or a lot. Taken together, these findings identify unique relationships between meaning and savoring concurrently and longitudinally, which were not empirically identified previously. Low arousal savoring is a particularly effective everyday savoring strategy for increasing meaning for adults, and having meaning in life is important for signalling being present and absorbed in the moment. Further, individuals who follow a life of meaning in adulthood will find their amplifying savoring strategies are more effective at creating intensely positive everyday events, compared to those who find a meaningful life less important.

\section{Strengths and Implications}

As only a few previous studies exist on the topic of savoring strategies (i.e., Bryant \& Veroff, 2007; Jose et al., 2012), this thesis accomplished several novel contributions to savoring research. With the creation of an abridged everyday savoring measure, this thesis tested the potential influence of development on the discrete cognitive-behavioural strategies proposed by savoring theory. Twenty short items represented each of the 10 original subscales of the 60 -item WOSC making the everyday savoring measure easier and quicker to administer to a wider age range (i.e. 463 adolescents, $M$ age $=13$ years; 1858 international adults, $M$ age $=40$ years) than the original version. With the everyday savoring measure, this study was the first to find savoring was a powerful predictor of wellbeing over time for a large group of adolescents and adults. This thesis bolstered the limited understanding of how to flourish by contributing valuable and timely efficacious savoring routes for the promotion of adolescent and adult flourishing, as well as presented a psychometrically robust measurement of everyday savoring for future research. 


\section{Everyday savoring}

The abridged scale was the first that investigated everyday savoring strategies, rather than those related to a discrete and relatively rare event, for instance, a good holiday (Bryant \& Veroff, 2007) or obtaining a diploma or winning the lottery (Quoidbach, et al., 2010). Certainly, individuals experience more everyday positive events than infrequent, discrete positive events. It follows that the opportunities for individuals to adopt the suggestions of the present research are greater, as are the opportunities to continue this research in context. Combined with the relative increased frequency of everyday savoring, a reliable abridged measure of savoring means researchers can gain information about savoring quicker with less risk of fatigue for the participants than is possible with the original 60-item WOSC.

Previous developmental research was conducted on the general capacity to savor (Bryant \& Veroff, 2007; Cafasso, et al., 1994), and less on how individuals specifically savor (Meehan, et al., 1993). Instead this study identified the specific everyday styles of adolescent and adult savoring from an abridged measure of the WOSC. From this work, the study established that $\mathrm{NZ}$ adolescents and international adults shared four similar everyday savoring styles. The interrelationships between savoring strategies were generally similar across groups except for one. The relationship between dampening and low arousal savoring appeared to become weaker from adolescence to young adulthood to middle adulthood. The strong positive relationship between dampening and low arousal in adolescence implied that low arousal acted more as a dampening strategy (i.e. reducing wellbeing), than as an amplifying strategy (i.e. enhancing wellbeing) in youth. This result emphasizes the importance of understanding adolescence as a unique developmental stage with unique goals and demands. It cannot simply be assumed that because adolescents and adults share a similar structure of savoring that they will also implement those savoring strategies identically.

The structure of everyday savoring withstood robust psychometric testing. The latent path modelling utilized in this thesis was able to account for measurement error and allow for more accurate estimate of autoregressive effects by including correlated errors over time. In addition, temporal tests of invariance ensured that savoring styles were consistent over time (i.e. longitudinally invariant). The tests of longitudinal invariance were important because they allowed any potential changes in reliability to be considered a result of theoretical processes rather than a result of measurement properties. From these tests, this research is able to confidently support the generalisation of the everyday savoring scale within normal populations, while recognizing the potential different ways the same style of savoring (i.e. low arousal savoring) might work across age groups. Considerable evidence indicates that everyday savoring 
strategies are universal, and the application of their measurement to diverse groups will continue to return valuable information for how individuals make the most of their everyday events.

\section{Everyday savoring and subjective wellbeing}

The present set of studies expanded previous investigations of concurrent relationships between savoring and hedonic wellbeing (e.g. Quoidbach, et al., 2010) by investigating savoring and eudaimonic wellbeing over time, in addition to hedonic wellbeing. When investigating the associations between everyday savoring and wellbeing, the suspected difference between adolescent and adult low arousal savoring was confirmed; low arousal savoring dampened wellbeing for adolescents and amplified wellbeing for adults. For adolescents, self-focused savoring was the strongest predictor of global wellbeing (i.e. both hedonia and eudaimonia), and it was the only predictor of positive affect over time. For adults, each amplifying savoring strategy uniquely related to each component of wellbeing. This research reiterates that findings from adult samples should not be extrapolated to adolescent populations, at least not without measured consideration and transparency.

These differences in low arousal savoring could be of particular interest to the area of happiness interventions and potentially to clinical psychology as well. Numerous self-help books and several clinical therapies (e.g. Acceptance Commitment Therapy and Dialectical Behavioural Therapy) have adopted mindfulness strategies as a central principle for improving wellbeing. However, applying mindfulness to adolescents may have adverse effects if, as this study suggests, they act more as dampeners than amplifiers. At bare minimum, the psycho-education for mindfulness interventions might require modifications before extending these interventions to adolescent populations. Further, mindfulness components of adolescent interventions need on going evaluation to ensure adolescents are indeed using these strategies efficaciously. This research cautions clinicians and counsellors to first ask the adolescent to reflect on their current use of these low arousal strategies and if necessary restructure the way they are currently implemented; for instance, by highlighting their positive utility.

Another possibility, and potentially more cost-effective route, might be to promote the one savoring strategy adolescents already favour for increasing their wellbeing. Adolescence is characterised by the establishment of a sense of self and formation of their own unique understanding of the world (Côté, 2004). Within this developmental stage, adolescents's self-focus savoring amplified the greatest number of wellbeing indicators, increased positive affect, and indirectly increased social connectedness, autonomy, personal growth, and environmental mastery (i.e. eudaimonic wellbeing). An adolescent intervention that targets these self-focus strategies would likely be the most effective savoring choice for improving adolescent wellbeing, 
and would require fewer resources to educate adolescents how to benefit from them since they already do. It would simply be a matter of maximising adolescents' savoring strengths to promote their global wellbeing, in other words, their flourishing.

On the other hand, this research indicates that multiple paths exist for improving adult wellbeing. Therefore a cost-effective approach would be to target the appropriate savoring strategies to increase specific deficits in wellbeing. An adult low in eudaimonia, for instance, would benefit from increasing their low arousal savoring, yet an adult low in frequent positive emotions would benefit more from developing their high arousal savoring strategies. These suggestions mirror mounting research advocating the need to match interventions to individual needs, weaknesses, and goals (Fordyce, 1977; Lyubomirsky, 2007; Sheldon \& Elliot, 1999). On the whole, this thesis pinpointed unique savoring routes to flourishing during adolescence and adulthood, which schools, counselling and health services, and individuals can consider exploiting.

\section{Limitations}

Although this study aimed to advance several avenues for understanding savoring and wellbeing, there were a number of limitations. First, this thesis relied solely on global self-report measures without verification from adjunct methods. The social sciences have always been sceptical of self-reporting data (e.g. Nisbett \& Wilson, 1977) with persuasive reasons for more rather than less scepticism related to self-reported measures of subjective wellbeing. The pressure of society to be happy might fuel participants' socially desirable responding and make it increasingly difficult for them to admit to unhappiness. Previous research, however, found that social desirability was a personality characteristic that enhanced wellbeing, rather than a response artefact that contributed error variance (Diener, Sandvik, Pavot, \& Gallagher, 1991). More recently researchers concluded that subjective instruments validly measure individual wellbeing after repeated evidence that subjective and objective measures of wellbeing converge (Sandvik, Diener, \& Seidlitz, 1993, 2009).

Well-documented limitations of global self-report measures have included their vulnerability to memory bias and relative inability to measure state conceptualisations of phenomena in comparison to experience sampling methods (for a review, Scollon, Kim-Prieto, \& Diener, 2003). When coping, for instance, was measured with global reports the cognitive coping styles were underreported while behavioural coping styles were over-reported. These findings imply that the cognitive savoring styles (e.g. dampening and self-focus) were underrepresented while the behavioural savoring styles (e.g. low and high arousal) were overrepresented here. Importantly the memory for an experience or feeling is not the same as 
the reality of it. Although the two overlap to a moderate/high degree, they do not equal one another. Experience sampling is measuring within-person correlations, which are better represent state effects that fluctuate more readily, while between-person correlations are better represent trait effects, which are more stable. In addition, the analyses presented in this thesis were meant to represent the average adolescent or adult, yet scholars have argued that by averaging across participants, the average individual might be lost (Conner, Tennen, Fleeson, \& Barrett, 2009).

Experience sampling methods have their weakness too, for example and especially important considering the present work, it can act as an intervention in itself. The mere task of asking people to reflect on a daily basis on how they savoured and how good they felt might encourage individuals to purposefully find more opportunities to savor, which was not the objective of this thesis. In addition, experience sampling is limited in the amount of information it can convey of a single construct. Whereas the present thesis asked about at least twenty savoring strategies and numerous wellbeing items, a recent experience sampling study only asked about three savoring items and one happiness item (Jose et al., 2012). Lastly, retrospective measures, although not exactly an accurate picture of reality, better predict behavioural choice. Research indicates that recalled emotion, for example, strongly predicts future desire for a similar experience, not the real-time emotion (Wirtz, Kruger, Scollon, \& Diener, 2003). Nonetheless, investigating adjunct approaches would have increased the confidence for the interpretations of the present findings and better illuminated state level processes; however, they were not feasible within the scope of this research.

Second, as noted throughout this thesis, the comparisons between adolescents and adults were made between different samples and studies (see Chapter 2, Discussion), which significantly challenged this author's ability to attribute savoring and wellbeing variability to age alone. Further, this study interpreted potential age effects with cross-sectional comparisons rather than longitudinal analyses. Previous studies have warned that the size of age effects often varies depending on whether a cross-sectional or longitudinal method was used (Kunzmann, et al., 2000; Pinquart, 2001). Cross-sectional studies evidence steeper declines in negative affect across age than longitudinal studies, whereas longitudinal studies evidence steeper inclines in positive affect than cross-sectional studies (Pinquart). Although this thesis did not test negative affect, considering the potential attenuating effect of cross-sectional methods on reported hedonia and eudaimonia is warranted.

Third, further methodological limitations probably biased sample composition. Due to convenience sampling $83 \%$ of the adult sample was female and $84 \%$ White-European. The adolescent sample was entirely from New Zealand, and included an overrepresentation of Māori 
compared to the general New Zealand population. The proposed differences in low arousal savoring, for instance, may in fact be attributable to gender rather than age effects. Chapter 2 attempted to control for this possibility by creating a sub-sample of adults with equal number of males and females before testing mean differences in savoring. Recall that the results showed neither the adolescent nor adult males and females differed in their low arousal savoring. A stratified sampling procedure, however, would have better controlled for these biases, especially with path models where I was unable to create equal gender groups for adults (e.g. Chapter 5). Caution, therefore, is recommended when generalising the findings from these biased samples to groups or individuals with different demographics.

Lastly, although particular chapters (Chapter 4 and 5) inferred causation on the basis of longitudinal data, without using experimental methods this thesis cannot unequivocally conclude that the observed relationships between everyday events, everyday savoring strategies, and wellbeing were causal in nature.

\section{Future Directions}

This thesis indicated several fruitful lines for continued research and development of savoring and wellbeing theories. As highlighted previously, individuals reported living their life with a focus on meaning more so than pleasure across the life-span from adolescence to adulthood. An interesting future research question might examine whether the particular types of meaning are consistent across adulthood and how they are related to an individual's choice of savoring strategies. Adolescence is a time of questioning and exploration, and it makes sense then that research on meaning has found that emerging adults (aged 18 to 20 years) report greater levels of searching for meaning in contrast to older adults who report greater levels of the presence of meaning (Steger, Oishi, \& Kashdan, 2009). Although five of the six meaning items on the Orientations to Happiness Scale appear to measure the presence of meaning, and number 17 may measure searching for meaning (Appendix J), a study with a validated measure of both the search and presence of meaning (i.e. Steger, et al., 2006) would be useful in future research. Perhaps low arousal savoring is associated with the presence of meaning, not searching for meaning. And it is possible that this association contributed to the finding that adolescent's low arousal savoring and eudaimonia were unrelated. Continued research would be able to unravel this mystery and contribute valuable information to understanding the associations between savoring and meaning across the lifespan.

Another logical next step in expanding the current savoring research would be to conduct an intervention study with adolescents. Adolescence is a particularly rewarding and challenging developmental stage. The OECD recently criticised New Zealand for having an 
unacceptable level of morbidity compared to other OECD nations during this life stage (OECD, 2009). In response, academics reported to the Prime Minsiter of New Zealand with suggestions that advised government how to roll-out and continually evaluate evidence-based life skills education (Gluckman, Low, \& Bay, 2011) and interventions (Fergusson, McNaughton, Hayne, \& Cunningham, 2011). In particular they encourage identifying strategies that can be disseminated universally and those that are specific to high-risk groups (Gluckman et al.), and suggested using randomised trials to evaluate the efficacy of interventions (Fergusson et al.). A randomised waitlist controlled study of adolescents who are taught specific savoring strategies and measured for increases in wellbeing would provide specific routes for positive health interventions. This study suggested that self-focused savoring strategies were a powerful predictor of feeling good and an indirect predictor of functioning well in adolescence. It follows that conducting a controlled study that helps facilitate these strategies and tests the validity of psycho-education for other strategies (e.g. low arousal savoring) is likely to provide important information for improving the health of New Zealand's adolescents.

This discussion of future directions is not exhaustive; there are numerous exciting avenues for continued research, including measuring real-time savoring in context and the impact of culture - to name a few. Recently, I contributed, with Bryant and Kluwe (2011), to an extensive discussion on future directions for investigating savoring. I refer the reader to this recent paper, 'Understanding the processes that regulate positive emotional experience' for a description of three more worthwhile and important avenues for developing savoring theory and research—namely, discriminating neuropsychological profiles associated with various savoring processes, developing viable methods for investigating mediational mechanisms involved in realtime savoring, and clarifying how individuals acquire different strategies to savor positive experiences across the life span.

\section{Conclusion}

The importance of emotional, cognitive, and functional wellbeing to positive health has previously been established. Yet, the unique relationships between positive events, everyday savoring, and hedonic and eudaimonic wellbeing are relatively less understood. No known studies had observed all of these variables within a single analysis to allow for a more realistic representation of savoring by both adolescents and adults. This thesis was able to fill some of these significant gaps. It found that the structure of everyday savoring is consistent across age and time. It also showed that how individuals choose to savor can have an important impact on global and specific types of wellbeing. This thesis warned that adult findings could not be automatically generalised to adolescents, and that adolescence is an important developmental 
stage that deserves recognition for its unique use of savoring. These contributions to the understanding of savoring provide promising domains for future research and application for making people's emotional and functional lives richer and more fulfilling. In effect this research illustrated that savoring is a powerful tool to combat the taking of everyday life for granted. Therefore, the final message from this thesis is to practice amplifying savoring as much and as often as possible, as it not only predicts positive emotions, social connection, and meaning, it also appears to activate the intensity of positive everyday events. 


\section{References}

Adams, T. B., Bezner, J. R., Drabbs, M. E., Zambarano, R. J., \& Steinhardt, M. A. (2000). Conceptualization and measurement of the spiritual and psychological dimensions of wellness in a college population. Journal of American College Health, 48(165-173).

Adler, M. G., \& Fagley, N. S. (2005). Appreciation: Individual differences in fiding value and meaning as a unique predictor of subjective well-being. Journal of Personality, 73(1), 80-114.

Algoe, S. B., Fredrickson, B. L., \& Chow, S. M. (2011). The future of emotions research within positive psychology. In K. M. Sheldon, T. B. Kashdan \& M. F. Steger (Eds.), Designing positive psychology: Taking stock and moving forward (pp. 115-132). New York, NY: Oxford University Press.

Anderson, J. C., \& Gerbing, D. W. (1988). Structural equation modeling in practice: A review and recommended two-step approach. Psychological Bulletin, 103(3), 411-423.

Arbuckle, J. L. (2009). AMOS 18 User's Guide. Crawfordville, FL: AMOS Development Corporation.

Argyle, M. (1999). Causes and correlates of happiness. In D. Kahneman, E. Diener \& N. Schwarz (Eds.), Well-being: The foundations of hedonic psychology (pp. 353-373). New York: Russell Sage Foundation.

Azmitia, M., Syed, M., \& Radmacher, K. (2008). On the intersection of personal and social identities: Introduction and evidence from a longitudinal study of emerging adults. In M. Azmitia, M. Syed \& K. Radmacher (Eds.), The intersections of personal and social identities. New Directions for Child and Adolescent Dvelopment (Vol. 120, pp. 1-16).

Baker, D. B., \& Benjanin, L. T. J. (2000). The affirmation of the scientist-practioner: A look back at Boulder. American Psychologist, 55(2), 241-247.

Baron, R. M., \& Kenny, D. A. (1986). The moderator-mediator variable distinction in social psychological research: Conceptual, strategic, and statistical considerations. Journal of Personality and Social Psychology, 51(6), 1173-1182.

Barrett, L. F., \& Russell, J. A. (1998). Independence and bipolarity in the structure of current affect. Journal of Personality and Social Psychology, 74(4), 967-984.

Baumeister, R. F., Bratslavsky, E., Finkenauer, C., \& Vohs, K. D. (2001). Bad is stronger than good. Review of General Psychology, 5(4), 323-370.

Ben-Shahar, T. (2007). Happier: Learn the secrets of daily joy and lasting fulfilment. New York, NY: McGraw-Hill. 
Bentler, P. M. (1990). Comparative fit indexes in structural models. Psychological Bulletin, 107(2), 238-246.

Bentler, P. M., \& Bonnett, D. G. (1980). Significance tests and goodness of fit in the analysis of covariance structures. Psychological Bulletin, 88(588-506).

Boehm, J. K., \& Lyubomirsky, S. (2009). The promise of sustainable happiness. In S. J. Lopez \& C. R. Snyder (Eds.), Oxford handbook of positive psychology (2 ed., pp. 667-677). New York, NY: Oxford University Press.

Bok, S. (2010). Exploring happiness: From Aristotle to brain science. New Haven: Yale University.

Bollen, K. A. (1989). Structural equations with latent variables. New York, NY: Wiley.

Boyle, P. A., Barnes, L. L., Buchman, A. S., \& Bennett, D. A. (2009). Purpose in life is associated with mortality among community-dwelling older persons. Psychosomatic Medicine, 71(5), $575-579$.

Bradburn, N. M. (1969). The structure of psychological well-being (Vol. 318).

Braungart, J. M., Plomin, R., DeFries, J. C., \& Fulker, D. W. (1992). Genetic influence on testerrrated infant temperament as assessed by Bayley's Infant Behavior Record: Nonadoptive and adoptive siblings and twins. Developmental Psychology, 28, 40-47.

Brennan, J. F. (2009). History and systems of psychology (6 ed.). Englewood Cliffs, NJ: Prentice Hall. Brickman, P., \& Campbell, D. T. (1971). Hedonic relativism and planning the good society. In M. H. Appley (Ed.), Adaptation level theory: A symposium (pp. 287-302). New York: Academic Press.

Brickman, P., Coates, D., \& Janoff-Bulman, R. (1978). Lottery winners and accident victims: Is happiness relative? Journal of Personality and Social Psychology, 36(8), 917-927.

Bryant, F. B. (1989). A four-factor model of perceived control: Avoiding, coping, obtaining, and savoring. Journal of Personality, 57(4), 773-797.

Bryant, F. B. (2003). Savoring beliefs inventory (SBI): A scale for measuring beliefs about savoring. Journal of Mental Health, 12(2), 175-196.

Bryant, F. B., Chadwick, E. D., \& Kluwe, K. (2011). Understanding the processes that regulate positive emotional experience: Unsolved problems and future directions for theory and research on savoring. International Journal of Wellbeing, 1(1), 107-126.

Bryant, F. B., \& Cvengros, J. A. (2004). Distinguishing hope and optimism: Two sides of a coin, or two separate coins? Journal of Social \& Clinical Psychology, 23(2), 273-302.

Bryant, F. B., Ericksen, C. L., \& DeHoek, A. (2008). Savoring. In S. J. Lopez (Ed.), Encyclopedia of positive psychology (Vol. 2, pp. 857-859). New York, NY: Wiley/Blackwell. 
Bryant, F. B., \& Morgan, L. (1986). The role of reminiscence in everyday life. . Paper presented at the 94th annual American Psychological Association convention.

Bryant, F. B., Smart, C. M., \& King, S. P. (2005). Using the past to enhance the present: Boosting happiness through positive reminiscence. Journal of Happiness Studies, 6, 227-260.

Bryant, F. B., \& Veroff, J. (1982). The structure of psychological well-being: A sociohistorical analysis. Journal of Personality and Social Psychology, 43(4), 653-673.

Bryant, F. B., \& Veroff, J. (1984). Dimensions of subjective mental health in American men and women. Journal of Health and Social Behavior, 25(2), 116-135.

Bryant, F. B., \& Veroff, J. (2007). Savoring: A new model of positive experience. Mahwah, NJ: Lawrence Erlbaum Associates, Inc.

Bryant, F. B., \& Yarnold, P. R. (1995). Principal-components analysis and exploratory and confirmatory factor analysis. In L. G. Grimm \& P. R. Yarnold (Eds.), Reading and understanding multivariate statistics (pp. 99-137). Washington, D.C.: American Psychological Association.

Byrne, B. M. (2001). Structural equation modeling with AMOS: Basic concepts, applications, and programming. Mahwah, NJ: Lawrence Erlbaum Associates.

Cafasso, L. L., Bryant, F. B., \& Jose, P. E. (1994). A scale for measuring children's savoring beliefs. Paper presented at the 102nd annual American Psychological Association convention.

Cahn, S. M., \& Vitrano, C. (2008). Happiness: Classic and contemporary readings in philosophy. New York: Oxford University Press.

Carstensen, L. L. (2006). The influence of a sense of time on human development. Science, 312(5282), 1913-1915.

Carstensen, L. L., Fung, H. H., \& Charles, S. T. (2003). Socioemotional selectivity theory and the regulation of emotion in the sencond half of life. Motivation and Emotion, 27(2), 102-123.

Carstensen, L. L., Pasupathi, M., Mayr, U., \& Nesselroade, J. R. (2000). Emotional experience in everyday life across the adult life span. Journal of Personality and Social Psychology, 79(4), 644655.

Carstensen, L. L., Turan, B., Scheibe, S., Ram, N., Ersner-Hershfield, H., Samanez-Larkin, R., et al. (2010). Emotional experience improves with age: Evidence based on over 10 years of experience sampling. Psychology and Aging, Advance online publication.

Carver, C. S., Scheier, M. F., \& Segerstrom, S. C. (2010). Optimism. Clinical Psychology Review, 30(7), 879-889. 
Charles, S. T., Luong, G., Almeida, D. M., Ryff, C. D., Sturm, M., \& Love, G. (2010). Fewer ups and downs: daily stressors mediate age differences in negative affect. The Journals of Gerontology: Series B: Psychological Sciences and Social Sciences, 65B(3), 279-286.

Chou, C. P., \& Bentler, P. M. (1995). Estimates and tests in structural equation modeling. In R. H. Hoyle (Ed.), Structural equation modeling: Concepts, issues and applications (pp. 37-55). Thousand Oaks: Sage Publishing.

Cohen, S., Doyle, W. J., Turner, R. B., Alper, C. M., \& Skoner, D. P. (2003). Emotional styel and susceptibility to the common cold. Psycholsomatic Medicine, 65(4), 652-657.

Cohn, M. A., Fredrickson, B. L., Brown, S. L., Mikels, J. A., \& Conway, A. M. (2009). Happiness unpacked: Positive emotions increase life satisfaction by building resilience. Emotion, 9(3), 361-368.

Conner, T.S., Tennen, H., Fleeson, W., \& Barrett, F. (2009). Experience sampling methods: A modern idiographic approach to personality research. Social and Personality Psychology Compass, 3, 1-22.

Cook, T. D., \& Campbell, D. T. (1979). Quasi-experimentation: Design and analysis issues for field settings. Chicago, US: Rand McNally.

Côté, J. E. (2004). Identity formation and self development in adolescence. In R. M. Lerner \& L. Steinberg (Eds.), Handbook of adolescent psychology (pp. 266-304). Hokoken, NJ: John Wiley $\&$ Sons.

Csikszentmihalyi, M. (1990). Flow: The psychology of optimal experience. New York, NY: Harper \& Row.

Csikszentmihalyi, M. (1991). Flow: The psychology of optimal experience. New York, NY: Harper Perennial.

Csikszentmihalyi, M. (1997). Finding flow: The psychology of engagement with everyday life. New York, NY: Basic Books.

Curran, P. J., West, S. G., \& Finch, J. F. (1996). The robustness of test statistics to nonnormality and specification error in confirmatory factor analysis. Psychological Methods, 1(1), 16-29.

Damon, W. (1995). Greater expectations: Overcoming the culture of indulgence in our homes and schools. New York, NY: Free Press.

Damon, W. (2004). What is Positive Youth Development? The ANNALS of the American Academy of political and social science, 591, 13-24.

Damon, W. (2008). The path to purpose: helping our children find their calling in life. New York, NY:

Free Press. 
Danner, D. D., Snowdon, D. A., \& Friesen, W. V. (2001). Positive emotions in early life and longevity findings from the nun study. Personality Processes and Individual Differences, 80(5), 804-813.

Davidson, R. J., \& Irwin, W. (1999). The funtional neuroanatomy of emotion and affective style. Trends in Cognitive Sciences, 3(1), 11-21.

Debats, D. L., Van der Lubbe, P. M., \& Wezeman, F. R. (1993). On the psychometric properties of the Life Regard Index (LRI): A measure of meaningful life: An evaluation in three independent samples based on the Dutch version. Personality and Individual Differences, 47(1105-1117).

Della Fave, A., Brdar, I., Freire, T., Vella-Brodrick, D., \& Wissing, M. P. (2011). The eudaimonic and hedonic components of happiness: Qualitative and quantitative findings. Social Indicators Research.

Delle Fave, A., \& Bassi, M. (2009). The contribution of diversity to happiness research. The Journal of Positive Psychology, 4(3), 205-207.

DeLongis, A., Folkman, S., \& Lazarus, R. S. (1988). The impact of daily stress on health and mood: Psychological and social resources as mediators. Journal of Personality and Social Psychology, 54(3), 486-495.

Diener, E. (1984). Subjective well-being. Psychological Bulletin, 95(3), 542-575.

Diener, E., \& Biswas-Diener, R. (2008). Happiness: Unlocking the mysteries of psychological wealth. New York: Wiley-Blackwell.

Diener, E., \& Emmons, R. A. (1985). The independence of positive and negative affect. Journal of Personality and Social Psychology, 47(5), 1105-1117.

Diener, E., Emmons, R. A., Larsen, R. J., \& Griffin, S. (1985). The satisfaction with life scale. Journal of Personality Assessment, 49(1), 71-75.

Diener, E., Lucas, R. E., \& Scollon, C. N. (2006). Beyond the hedonic treadmill. American Psychologist, 61(4), 305-314.

Diener, E., Lucas, R. E., \& Scollon, C. N. (2009). Beyond the hedonic treadmill:Revising the adaptation theory of well-being. Social Indicators Research Series, 37, 103-118.

Diener, E., Nickerson, C., Lucas, R. E., \& Sandvik, E. (2002). Dispositional affect and job outcomes. Social Indicators Research, 59(3), 229-259.

Diener, E., Sandvik, E., Pavot, W., \& Gallagher, D. (1991). Social Indicators Research. 24(1), 3556.

Diener, E., Sapyta, J. J., \& Suh, E. (1998). Subjective well-being is essential to well-being. Psychological Inquiry, 9(1), 33-37. 
Diener, E., Scollon, C. N., \& Lucas, R. E. (2009). The evolving concept of subjective well-being: The multifaceted nature of happiness. Social Indicators Research Series, 39, 67-100.

Diener, E., \& Seligman, M. E. P. (2002). Very happy people. Psychological Science, 13(1), 81-84.

Diener, E., \& Suh, E. (1997). Measuring quality of life: Economic, social and subjective indicators. Social Indicators Research, 40(189-216).

Diener, E., \& Suh, E. M. (1998). Subjective well-being and age: An international analysis. In K. W. Schaie \& M. P. Lawton (Eds.), Annual review of gerontology and geriatrics (Vol. 8, pp. 304324). New York: Springer.

Diener, E., Suh, E. M., Lucas, R. E., \& Smith, H. L. (1999). Subjective well-being: Three decades of progress. Psychological Bulletin, 125(2), 276-302.

Ein-Dor, T., Mikulincer, M., \& Shaver, P. R. (2011). Attachment insecurities and the processing of threat-related information: Studying the schemas involved in insecure people's coping strategies. Journal of Personality and Social Psychology, 10(1), 78-93.

Emmons, R. A., \& McCullough, M. E. (2003). Counting blessings versus burdens: an experimental investigation of gratitude and subjective well-being in daily life. Journal of Personality and Social Psychology, 84(2), 237-389.

Emmons, R. A., \& Shelton, C. M. (2002). Gratitude and the science of positive psychology. In C. R. Snyder (Ed.), Handbook of positive psychology (Vol. xviii, pp. 459-471). New York, NY: Oxford University Press.

Extremera, N., Ruiz-Aranda, D., Pineda-Galan, C., \& Salguero, J. M. (2011). Emotional intelligence and its relations with hedonic and eudaimonic well-being: A prospective study. Personality and Individual Differences, 51, 11-16.

Fergusson, D., McNaughton, S., Hayne, H., \& Cunningham, C. (2011). From evidence to policy, programmes and interventions. Auckland, NZ: Office of the Prime Minister's Science Advisory Committe.

Field, A. (2009). Discovering statistics using SPSS (3 ed.). London: Sage.

Fledderus, M., Bohlmeijer, E. T., Smit, F., \& Westerhof, G. J. (2010). American Journal of Public Health, 100(12), 2372-2378.

Folkman, S., \& Lazarus, R. S. (1980). An analysis of coping in a middle-aged community sample. Journal of Health and Social Behavior, 21(3), 219-239.

Folkman, S., \& Lazarus, R. S. (1985a). If it changes it must be a process: Study of emotion and coping during three stages of a college exam. Journal of Personality and Social Psychology, 48(1), 150-170. 
Folkman, S., \& Lazarus, R. S. (1985b). If it changes it must be a process: Study of emotion and coping during three stages of a college examination. Journal of Personality and Social Psychology, 48(1), 150-170.

Folkman, S., \& Lazarus, R. S. (1988). Coping as a mediator of emotion. Journal of Personality and Social Psychology, 54(3), 466-475.

Folkman, S., Lazarus, R. S., Dunkel-Schetter, C., DeLongis, A., \& Gruen, R. J. (1986). Dynamics of a stressful encounter: Cognitive appraisal, coping, and encounter outcomes. Journal of Personality and Social Psychology, 50(5), 992-1003.

Folkman, S., Lazarus, R. S., Gruen, R. J., \& DeLongis, A. (1986). Appraisal, coping, health status, and psychological symptoms. Journal of Personality and Social Psychology, 50(3), 571-579.

Folkman, S., \& Moskowitz (2000). Positive affect and the other side of coping. American Psychologist, 55(6), 647-654.

Fordyce, M. W. (1977). Development of a program to increase personal happiness. Journal of Counseling Psychology, 24(6), 511-521.

Frankl, V. E. (1963). Man's search for meaning, an introduction to logotherapy. Oxford, England: Washington Square Press.

Fredrickson, B. L. (1998). What good are positive emotions? Review of General Psychology, 2, 300319.

Fredrickson, B. L. (2001). The role of positive emotions in positive psychology: The broadenand-build theory of positive emotions. American Psychologist, 56(3), 218-226.

Fredrickson, B. L. (2002). Positive emotions. In C. R. Snyder \& S. J. López (Eds.), Handbook of positive psychology (pp. 120-134). Oxford: Oxford University Press.

Fredrickson, B. L. (2004). The broaden-and-build theory of positive emotions. The Philosophical Transactions of the Royal Society B: Biological Sciences, 359(1367-1377.).

Fredrickson, B. L. (2005a). The broaden-and-build theory of positive emotions. In F. A. Huppert, N. Baylis \& B. Keverne (Eds.), The science of well-being (pp. 217-238). New York: Oxford University Press.

Fredrickson, B. L. (2005b). Positive emotions. In C. R. Snyder \& S. J. Lopez (Eds.), Handbook of positive psychology (pp. 120-134). New York: Oxford University Press.

Fredrickson, B. L., \& Branigan, C. (2001). Positive emotions. In T. Mayne \& G. Bonanno (Eds.), Emotions: Current issues and future directions (pp. 123-151). New York: Guilford.

Fredrickson, B. L., Cohn, M. A., Coffey, K. A., Pek, J., \& Finkel, S. M. (2008). Open hearts build lives: Positive emotions, induced through loving-kindness meditation, build 
consequential personal resources. Journal of Personality and Social Psychology, 95(5), 10451062.

Fredrickson, B. L., \& Losada, M. F. (2005). Positive affect and the complex dynamics of human flourishing. American Psychologist, 60(7), 678-686.

Fredrickson, B. L., Tugade, M. M., Waugh, C. E., \& Larkin, G. R. (2003). What good are positive emotions in crisis? A prospective study of resilience and emotions following the terrorist attacks on the United States on September 11th, 2001. Journal of Personality and Social Psychology, 84(2), 365-376.

Froh, J. J., \& Bono, G. (Eds.). (2011) Encyclopedia of Adolecence. New York: Springer.

Froh, J. J., Emmons, R. A., Card, N. A., Bono, G., \& Wilson, J. (in press). Gratitude and the reduced costs of materialism in adolescents. Journal of Happiness Studies.

Froh, J. J., Sefick, W. J., \& Emmons, R. A. (2008). Counting blessings in early adolescents: An experimental study of gratitiude and subjective well-being. Journal of School Psychology, 46, 213-233.

Froh, J. J., Yurkewicz, C., \& Kashdan, T. B. (2009). Gratitude and subjective well-being in early adolescence: Examining gender differences. Journal of Adolescence, 32(3), 633-650.

Gable, S. L., Reis, H. T., Impett, E. A., \& Asher, E. R. (2004). What do you do when things go right? The intrapersonal and interpersonal benefits of sharing positive events. Journal of Personality and Social Psychology, 87(2), 228-245.

Garland, E. L., Fredrickson, B. L., Kring, A. M., Johnson, D. P., Meyer, P. S., \& Penn, D. L. (2010). Upward spirals of positive emotions counter downward spirals of negativity: Insights from the broaden-and-build theory and affective neuroscience on the treatment of emotion dysfunctions and deficits in psychopathology. Clinical Psychology Review, 30(7), 849-964.

Gluckman, P., Low, F., \& Bay, J. (2011). The value of evidence-based life skills education. Auckland, NZ: Office of the Prime Minsiter's Advisory Committee.

Gottman, J. M. (1994). What predicts divorce? Hillsdale NJ: Lawrence Erlbaum Associates.

Gottman, J. M., Coan, J., Carrere, S., \& Swanson, C. (1998). Predicting marital happiness and stability from newlywed interactions. Journal of Marriage \& the Family, 60(1), 5-22.

Howell, D. C. (2009). Statistical methods for psychology (7 ed.). Belmont, CA: Wadsworth Publishing.

Hoyle, R. H., \& Smith, G. T. (1994). Formulating clinical research hypotheses as structural equation models: A conceptual overview. Journal of Consulting and Clinical Psychology, 62(3), 429-440. 
Hu, L.-T., \& Bentler, P. M. (1999). Cutoff criteria for fit indexes in covariance structure analysis: Conventional criteria versus new alternatives. Structural Equation Modeling, 6(1), 1-55.

Huta, V., \& Ryan, R. M. (2010). Pursuing pleasure or virtue: The differential and overlapping well-being benefits of hedonic and eudaimonic motives. Journal of Happiness Studies.

Isen, A. M. (1987). Positive affect, cognitive processes, and social behavior. Advances in Experimental Social Psychology, 20, 203-253.

Isen, A. M. (1999). Positive affect. Dalgleish, Tim.

Isen, A. M. (2001). An influence of positive affect on decision making in complex situations: Theoretical issues with practical implications. Journal of Consumer Psychology, 11, 75-85.

Isen, A. M., \& Daubman, K. A. (1984). The influence of affect on categorization. Journal of Personality and Social Psychology, 47, 1206-1217.

Isen, A. M., Rosenzweig, A. S., \& Young, M. J. (1991). The influence of positive affect on clinical problem solving. Medical Decision Making, 11, 221-227.

Jahoda, M. (1958). Current concepts of positive mental health. New York, NY: Basic Books.

Jefferson, T. (1776, July 4). The Unanimous Declaration of the Thirteen United States of America from http://www.archives.gov/exhibits/charters/declaration.html.

Jones, H. A. (1966). The pursuit of happiness. Ithaca: Cornell University Press.

Jöreskog, K. G., \& Sörbom, D. (1982). Recent developments in structural equation modeling. journal of Marketing Research, 19, 404-416.

Jose, P. E. (2008). ModGraph-I: A programme to compute cell means for the graphical display of moderational analyses: The internet version, Version 2.0. Victoria University of Wellington, Wellington, New Zealand. Retrieved June, 2010, from http://www.victoria.ac.nz/psyc/staff/pauljose-files/modgraph/modgraph.php

Jose, P. E. (2009). The measurement of the effects of positive events. Unpublished manuscript, Victoria University of Wellington, Wellington, New Zealand.

Jose, P. E., Cafasso, L. L., \& D'Anna, C. A. (1994). Ethnic group differences in children's coping strategies. Sociological Studies of Children, 6, 25-53.

Jose, P. E., D'Anna, C. A., Cafasso, L. L., Bryant, F. B., Chiker, V., Gein, N., et al. (1998). Stress and coping among Russian and American early adolescents. Developmental Psychology, 64(4), 757-769.

Jose, P.E., \& Huntsinger, C.S. (2005). Moderation and mediation effects of coping by Chinese American and European American adolescents. The Journal of Genetic Psychology, 166, 1644. 
Jose, P. E., Lim, B. T., \& Bryant, F. B. (2011). Does savoring increase happiness? A daily diary study. Manuscript submitted for publication.

Kahneman, D., Diener, E., \& Schwarz, N. (1999). Well-being: The foundations of hedonic psychology. New York: Russell Sage Foundation.

Kamvar, S., Mogilner, C., \& Aaker, J. (2009). The meaning(s) of happiness. Research Paper Series Standford Graduate School of Business.

Kashdan, T. B., Biswas-Diener, R., \& King, L. (2008). Reconsidering happiness: The costs of disntinguishing between hedonics and eudaimonia. The Journal of Positive Psychology, 3(4), 219-233.

Kashdan, T. B., Breen, W. E., \& Julian, T. (2010). Everyday strivings in war veterans with Posttraumatic Stress Disorder: Suffering from a hyper-focus on avoidance and emotion. Behavior Therapy, 41(3), 350-363.

Kennedy, P. (Writer) (2011). Say no to happiness, with: Gretchen Rubin, Daniel Polish, Todd Kashdan, \& Jordan B. Peterson. In F. Faulk (Producer), IDEAS. Canada: CBC Radio.

Kenny, D. A. (1979). Correlation and causality. New York, NY: Wiley.

Keyes, C. L. M. (2002). The mental health continuum: From languishing to flourishing in life. Journal of Health and Social Behavior, 43(2), 207-222.

Keyes, C. L. M. (2003). Complete mental health: An agenda for the 21 st century Flourishing: Positive psychology and the life well-lived (pp. 293-312). Washington, DC: American Psychological Association.

Keyes, C. L. M. (2005a). Mental Illness and/or Mental Health? Investigating Axioms of the Complete State Model of Health. Journal of Consulting and Clinical Psychology, 73(3), 539-548.

Keyes, C. L. M. (2005b). The subjective well-being of America's youth: Toward a comprehensive assessment. Adolescent \& Family Health, 4, 3-11.

Keyes, C. L. M. (2006). Mental Health in Adolescence: Is America's Youth Flourishing? American Journal of Orthopsychiatry, 76(3), 395-402.

Keyes, C. L. M. (2007). Promoting and Protecting Mental Health as Flourishing: A Complementary Strategy for Improving National Mental Health. American Psychologist, 62(2), 95-108.

Keyes, C. L. M., \& Annas, J. (2009). Feeling good and functioning well: Distinctive concepts in ancient philosophy and contemporary science. The Journal of Positive Psychology, 4(3), $197-$ 201. 
Keyes, C. L. M., \& Lopez, S. J. (2005). Toward a science of mental health. In C. R. Snyder \& S. J. Lopez (Eds.), Handbook of positive psychology (pp. 45-59). New York: Oxford University Press.

Keyes, C. L. M., Shmotkin, D., \& Ryff, C. D. (2002). Optimizing well-being: The empirical encounter of two traditions. Journal of Personality and Social Psychology, 82(6), 1007-1022.

King, L. A., Hicks, J. A., Krull, J. L., \& Del Gaiso, A. K. (2006). Positive affect and the experience of meaning in life. Journal of Personality and Social Psychology, 90(1), 197-196.

Kishton, J. M., \& Widaman, K. F. (1994). Unidimensional versus domain representative parceling of questionnaire items: An empirical example. Educational and Psychological Measurement, 54, 757-765.

Kleinke, C. L., Peterson, T. R., \& Rutledge, T. R. (1998). Effects of self-generated facial expressions on mood. Journal of Personality and Social Psychology, 74, 272-279.

Kline, R. B. (2005). Principles and practice of structural equation modeling. New York, NY: Guilford Press.

Koole, S. L. (2009). The psychology of emotion regulation: An integrative review. Cognition \& Emotion, 23(1), 4-41.

Kukutai, T. (2008). Ethnic self-prioritisation of dual and multi-ethnic youth in New Zealand. Wellington: Statistics New Zealand.

Kunzmann, U., Little, T. D., \& Smith, J. (2000). Is age-related stability of subjective well-being a paradox? Cross-sectional and longitudinal evidence from the Berlin Aging Study. Psychology and Aging, 15(3), 511-526.

Kurtz, J. L. (2008). Looking to the future to appreciate the present: The benefits of perceived temporal scarcity. Psychological Science, 19(1238-1241).

Lambie, J. A., \& Marcel, A. J. (2002). Consciousness and the variaties of emotion experience: A theoretical Framework. Psychological Review, 109(2), 219-259.

Langston, C. A. (1994). Capitalizing on and coping with daily-life events: Expressive responses to positive events. Journal of Personality and Social Psychology, 67(6), 1112-1125.

Layard, R. (2006). Happiness: Lessons from a new science. London: Penguin.

Levertov, D. (1964). O taste and see: New poems. New York, NY: New Directions.

Lindberg, T. L. (2004). Culture and savoring of positive experiences. The University of British Columbia.

Little, T. D., Cunningham, W. A., Shahar, G., \& Widaman, K. F. (2002). To parcel or not to parcel: Exploring the question, weighing the merits. Structural Equation Modeling, 9(2), 151173. 
Lucas, R. E. (2007). Adaptation and the set-point model of subjective well-being: Does happiness change after major life events? Current Directions in Psychological Science, 16(2), 75 79.

Lucas, R. E., \& Gohm, C. L. (2000). Age and sex differences in subjective well-being across cultures. In E. Diener \& E. M. Suh (Eds.), Culture and subjective well-being (Vol. 3, pp. 291 317). Cambridge, MA: The MIT Press.

Lykken, D. (1999). Happiness: What studies on twins show us about nature, nurture, and the happiness set point. New York, NY: Goldent Books.

Lykken, D. (2007). Comment on Diener, Lucas, and Scollon (2006). American Psychologist, 611612.

Lykken, D., \& Tellegen, A. (1996). Happiness is a stochastic phenomenon. Psychological Science, 7(3), 186-189.

Lyubomirsky, S. (2001). Why are some people happier than others? The role of cognitive and motivational processes in well-being. American Psychologist, 56(3), 239-249.

Lyubomirsky, S. (2007). The how of happiness. New York: Penguin Press.

Lyubomirsky, S. (2008). The how of happiness. New York: Penguin Press.

Lyubomirsky, S. (2009). The how, what, when, and why of happiness: Mechanisms Underlying the success of positive interventions. Paper presented at the The First World Congress on Positive Psychology.

Lyubomirsky, S., King, L., \& Diener, E. (2005). The benefits of frequent positive affect: Does happiness lead to success? Psychological Bulletin, 131(6), 803-855.

Lyubomirsky, S., \& Lepper, H. S. (1999). A measure of subjective happiness: Preliminary reliability and construct validitation. Social Indicators Research, 46(2), 137-155.

Lyubomirsky, S., Sheldon, K., \& Schkade, D. (2005). Pursuing Happiness: The Architecture of Sustainable Change. Review of General Psychology, 9(2), 111-131.

MacKinnon, D. P. (2008). Introduction to statistiacl mediation analysis. New York, NY: Lawernce Erlbaum Associates.

Martin, G. M. (2005). Epigenetic drift in aging identical twins. Proceedings of the National Academy of Sciences, 102(30), 10413-10414.

Maslow, A. H. (1954). Motivation and personality. New York: Harper \& Row.

Maslow, A. H. (1962). Toward a psychology of being. Princeton, NJ: D Van Nostrand.

Mauss, I. B., Tamir, M., Anderson, C. L., \& Savino, N. S. (2011). Can seeking happiness make people happy? Paradoxical effects of valuing happiness. Emotion, Advance online publication. 
McCrae, R. R., \& Costa, P. T. (1994). The stability of personality: Observations and evaluations. Current Directions in Psychological Science, 3(173-175).

McCullough, M. E. (2002). Savoring life, past and present: Explaining what hope and gratitude share in common. Psychological Inquiry, 13(4), 302-304.

McCullough, M. E., Emmons, R. A., \& Tsang, J.-A. (2002). The grateful disposition: A conceptual and empirical topography. Journal of Personality and Social Psychology, 82(1), 112127.

McCullough, M. E., Tsang, J.-A., \& Emmons, R. A. (2004). Gratitude in intermediate affective terrain: Links of grateful moods to individual differences and daily emotional experience. Journal of Personality and Social Psychology, 86(2), 295-309.

McMahon, D. M. (2006). Happiness: A history. New York, NY: Grove Press.

Meehan, M. P., Durlak, J. A., \& Bryant, F. B. (1993). The relationship of social support to perceived control and subjective mental health in adolescents. Journal of Community Psychology, 21(1), 49-55.

Moffit, T. E. (1993). Adolescence-limited and life course persistent antisocial behavior: A developmental taxonomy. Psychologica Review, 100, 674-701.

Moffit, T. E., Caspi, A., Harrington, H., \& Milne, B. J. (2002). Males on the life-course-persistent and adolescence-limited antisocial pathways: Follow-up at age 26 years. Devleopment and Psychopathology, 14(1), 179-207.

Mogilner, C., Kamvar, S., \& Aaker, J. (2011). The shifting meaning of happiness. Social Psychological and Personality Science, 2(4), 395-402.

Nes, R. B. (2011). Happiness in behaviour genetics: Findings and implications. Journal of Happiness Studies, 11(3), 369-381.

Nettle, D. (2005). Happiness: The science behind your smile. New York: Oxford University Press.

Niederhoffer, K. B., \& Pennebaker, J. W. (2005). Sharing one's story: On the benefits of writing or talking about emotional experience. In C. R. Snyder \& S. J. Lopez (Eds.), Handbook of positive psychology (pp. 573-583). New York: Oxford University Press.

Nisbett, R. E., \& Wilson, T. D. (1977). Telling more than we can know: Verbal reports on mental processes. Psychological Review, 84, 231-259.

Nolen-Hoeksema, S., \& Girgus, J. S. (1994). The emergence of gender differences in depression during adolescence. Psychological Bulletin, 115(3), 424-443.

Nozick, R. (1974). Anarchy, state, and utopia. Oxford: Blackwell.

OECD (2009). Doing Better for Children. 
Oishi, S., Diener, E., \& Lucas, R. E. (2007). The optimum level of well-being: Can people be too happy? Perspectives on Psychological Science, 2(4), 346-360.

Oishi, S., \& Koo, M. (2008). Two new questions about happiness: "Is happiness good?" and "Is happier better?". In M. Eid \& R. J. Larsen (Eds.), Handbook of subjective well-being (pp. 290306). New York: Oxford University Press.

Oishi, S., \& Kurtz, J. L. (2011). The positive psychology of positive emotions: An avuncular view. In K. M. Sheldon, T. B. Kashdan \& M. F. Steger (Eds.), Designing positive psychology: Taking stock and moving forward (pp. 101-114). New York, NY: Oxford University Press.

Palgi, Y., Shrira, A., Ben-Ezra, M., Cohen-Fridel, S., \& Bodner, E. (2011). The relationships between daily optimism, daily pessimism, and affect differ in young and old age. Personality and Individual Differences, 50(8), 1294-1299.

Park, N., Peterson, C., \& Ruch, W. (2009). Orientations to happiness and life satisfaction in twenty-seven nations. The Journal of Positive Psychology, 4(4), 273-279.

Pavot, W., \& Diener, E. (2008). The Satisfaction With Life Scale and the emerging construct of life satisfaction. The Journal of Positive Psychology, 3(2), 137-152.

Pavot, W., \& Diener, E. (2009). Review of the Satisfaction With Life Scale. Social Indicators Research Series, 39, 101-117.

Pavot, W., Diener, E., \& Suh, M. E. (1998). The temporal satisfaction with life scale. Journal of Personality Assessment, 7(2), 340-354.

PBS (Producer). (2007, June 16 2011) Epigenetics: Environmental factors can alter the way our genes are expressed making even identical twins different. Podcast retrieved from http://www.pbs.org/wgbh/nova/body/epigenetics.html.

Peterson, C., Park, N., \& Seligman, M. E. P. (2005). Orientations to happiness and life satisfaction: The full life versus the empty life. Journal of Happiness Studies, 6, 25-41.

Peterson, C., Ruch, W., Beermann, U., Park, N., \& Seligman, M. E. P. (2007). Strengths of character, orientations to happiness, and life satisfaction. The Journal of Positive Psychology, 2(3), 149-156.

Pinquart, M. (2001). Age differences in perceived positive affect, negative affect, and affect balance in middle and old age. Journal of Happiness Studies, 2(3), 375-405.

Plomin, R., \& Nesselroade, J. R. (1990). Behavioral genetics and personality change. Journal of Personality, 58(1), 191-220.

Portela, A., \& Esteller, M. (2010). Epigenetic modifications and human disease. Nature Biotechnology, 28(1057-1068). 
Price, J. M., \& Lento, J. (2001). The nature of child and adolecent vulnerability: History and definitions. In R. E. Ingram \& J. M. Price (Eds.), Vulnerability to Psychopathology (pp. 2038). New York, NY: Guilford Press.

Proctor, C. L., Linley, P. A., \& Maltby, J. (2009). Youth life satisfaction: A review of the literature. Journal of Happiness Studies.

Quintana, S. M., \& Maxwell, S. E. (1999). Implications of recent developments in structural equation modeling for counseling psychology. Counseling Psychologist, 27, 485-527.

Quoidbach, J., Berry, E. V., Hansenne, M., \& Mikolajczak, M. (2010). Positive emotion regulation and well-being: Comparing the impact of eight savoring and dampening strategies. Personality and Individual Differences, 49, 368-373.

Reis, H. T., Smith, S. M., Carmichael, C. L., Caprariello, P. A., Tsai, F. F., Rodrigues, A., et al. (2010). Are you happy for me? How sharing positive events with others provides personal and interpersonal benefits. Journal of Personality and Social Psychology, 99(2), 311329.

Riediger, M., Schmiedek, F., Wagner, G., \& Lindenberger, U. (2009). Seeking pleasure and seeking pain: Differences in prohedonic and contra-hedonic motivation from adolescence to old age. Psychological Science, 20, 1529-1535.

Roberts, M. C., Brown, K. J., Johnson, R. J., \& Reinke, J. (2005). Positive psychology for children: Development, prevention and promotion. In C. R. Snyder \& S. J. Lopez (Eds.), Handbook of positive psychology (pp. 663-675). New York: Oxford University Press.

Rosenthal, R. (1991). Metha-analytic procedures for social research (Revised ed.). Newbury Park, CA: Sage.

Ruch, W., Harzer, C., Proyer, R. T., Park, N., \& Peterson, C. (2011). Ways to happiness in German-speaking countries. European Journal of Psychological Assessment, 26(3), 227-234.

Ryan, R. M., \& Deci, E. L. (2001). On happiness and human potentials: A review of research on hedonic and eudaimonic well-being. Annual Review of Psychology, 52, 141-166.

Ryan, R. M., \& Huta, V. (2009). Wellness as healthy functioning or wellness as happiness: The importance of eudaimonic thinking (Response to the Kashdan et al. and Waterman discussion). The Journal of Positive Psychology, 4(3), 202-204.

Ryff, C. D. (1989). Happiness is everything, or is it? Explorations on the meaning of psychological well-being. Journal of Personality and Social Psychology, 57(6), 1069-1081.

Ryff, C. D., \& Keyes, C. L. M. (1995). The structure of psychological well-being revisted. Journal of Personality and Social Psychology, 69(4), 719-727. 
Ryff, C. D., \& Singer, B. (1998). The contours of positive human health. Psychological Inquiry, 9(1), $1-28$.

Ryff, C. D., \& Singer, B. H. (2008). Know thyself and become what you are: A eudaimonic approach to psychological well-being. Journal of Happiness Studies, 9(2), 13-39.

Sandvik, E., Diener, E., \& Seidlitz, L. (1993). Subjective well-being: The convergence and stability of self-report and non-self-report measures. Journal of Personality, 61(317-342).

Sandvik, E., Diener, E., \& Seidlitz, L. (2009). Subjective well-being: The convergence and stability of self-report and non-self-report measures. Social Indicators Research Series, 39, 119-137.

Schueller, S. M., \& Seligman, M. E. P. (2010). Pursuit of pleasure, engagement, and meaning: Relationships to subjective and objective measures of well-being. The Journal of Positive Psychology, 5(4), 253-263.

Scollon, C.N., Kim-Prieto, C., \& Diener, E. (2003). Experience sampling: Promises and pitfalls, strengths and weaknesses. Journal of Happiness Studies, 4, 5-34.

Seligman, M. E. P. (1992). Learned optimism. New York, NY: Pocket Books.

Seligman, M. E. P. (2002). Authentic happiness: Using the new positive psychology to realize your potential for lasting fulfillment. New York, NY: Free Press.

Seligman, M. E. P., \& Csikszentmihalyi, M. (2000). Positive psychology: An introduction. American Psychologist, 55(1), 5-14.

Seligman, M. E. P., Parks, A. C., \& Steen, T. A. (2004). A balanced psychology and a full life. Philosophical Transactions of the Royal Society B, 359, 1379-1381.

Seligman, M. E. P., Rashid, T., \& Parks, A. C. (2006). Positive Psychotherapy. American Psychologist, 774-788.

Sheldon, K. M., \& Elliot, A. J. (1999). Goal striving, need satisfaction, and longitudinal wellbeing: The self-concordance model. Journal of Personality and Social Psychology, 76, 546-557.

Sheldon, K. M., \& Lyubomirsky, S. (2006). How to increase and sustain positive emotion: The effects of expressing gratitude and visualizing best possible selves. Journal of Positive Psychology, 1(2), 73-82.

Sirgy, M. J., \& Wu, J. (2009). The pleasant life, the engaged life, and the meaningful life: What about the balanced life? Journal of Happiness Studies, 10(2), 183-196.

Steger, M. F., Frazier, P., \& Oishi, S. (2006). The Meaning in Life Questionnaire: Assessing the presence of and search for meaning in life. Journal of Counseling Psychology, 53(1), 80-93. 
Steger, M. F., Oishi, S., \& Kashdan, T. B. (2009). Meaning in life across the life span: Levels and correlates of meaning in life from emerging adulthood to older adulthood. The Journal of Positive Psychology, 4(1), 43-52.

Steiger, J. H. (1990). Structural model evaluation and modification: An interval estimation approach. Multivariate Behavioral Research, 25, 173-180.

Stone, A., Schwartz, J. E., Broderick, J. E., \& Deaton, A. (2010). A snapshot of the age distribution of psychological well-being in the United States. Proceedings of the National Academy of Sciences, 107(22), 9985-9990.

Suldo, S., \& Shaffer, E. J. (2008). Looking beyond psychopathology: The dual-factor model of mental health in youth. School Psychology Review, 37(1), 52-68.

Suldo, S., Thalji, A., \& Ferron, J. (2011). Longitudinal academic outcomes predicted by early adolescents' subjective well-being, psychopathology, and mental health status yielded from a dual factor model. The Journal of Positive Psychology, 6(1), 17-30.

Tabachnick, B. G., \& Fidell, L. S. (2001). Using multivariate statistics (4 ed.). Boston, MA: A Pearson

Tabachnick, B. G., \& Fidell, L. S. (2007). Using multivariate statistics. Boston, MA: Allyn \& Bacon.

Tamir, M. (2009). What do people want to feel and why? Pleasure and utility in emotion regulation. Current Directions in Psychological Science, 18, 101-105.

Tamir, M., Mitchell, C., \& Gross, J. J. (2008). Hedonic self-control predicts good adjustment, less pathology, better grades, and interpersonal success. Psychological Science, 19, 324-328.

Tellegen, A., Lykken, D., Bouchard, T. J., Wilcox, K. J., Segal, N. L., \& Rich, S. (1988). Personality similarity in twins reared apart and together. Journal of Personality and Social Psychology, 54, 1031-1039.

Tugade, M. M., \& Fredrickson, B. L. (2004). Resilient individuals use positive emotions to bounce back from negative emotional experiences. Journal of Personality and Social Psychology, 86(2), 320-333.

Tugade, M. M., Fredrickson, B. L., \& Barrett, L. F. (2004). Psychological resilience and positive emotional granularity: Examining the benefits of positive emotions on coping and health. Journal of Personality. Special Issue: Emotions, Personality, and Health, 72(6), 1161-1190.

Vella-Brodrick, D., Park, N., \& Peterson, C. (2009). Three ways to be happy: Pleasure, engagement, and meaning--findings from Australian and US samples. Social Indicators Research, 90, 165-179.

Wachowski, A., \& Wachowski, L. (Writer) (1999). The Matrix. U.S.A.: Warner Bros. Pictures and Village Road Pictures. 
Waterman, A. S. (1984). The psychology of individualism (pp. 359). New York: Praeger Publishers.

Waterman, A. S. (1993). Two conceptions of happiness: Contrasts of personal expressiveness (eudaimonia) and hedonic enjoyment. Journal of Personality and Social Psychology, 64(4), 678691.

Waterman, A. S. (2008). Reconsidering happiness: A eudaimonist's perspective. The Journal of Positive Psychology, 3(4), 234-252.

Waterman, A. S., Schwartz, S. J., \& Conti, R. (2008). The implications of two conceptions of happiness (hedonic enjoyment and eudaimonia) for the understanding of intrinsic motivation. Journal of Happiness Studies, 9, 41-79.

Watson, D. (2000a). Mood and temperament. New York: Guilford Press.

Watson, D. (2000b). Mood and temperament. Emotions and social behavior. New York, NY, US: Guilford Press, 340.

Watson, D. (2005). Positive affectivity: The disposition to experience pleasurable emotional states. In C. R. Snyder \& S. J. Lopez (Eds.), Handbook of positive psychology (pp. 106-119). New York: Oxford University Press.

Watson, D., Lee, A. C., \& Tellegen, A. (1988). Development and validation of brief measures of positive and negative affect: The PANAS scales. Journal of Personality and Social Psychology, 54(6), 1063-1070.

Waugh, C. E., \& Fredrickson, B. L. (2006). Nice to know you: Positive emotions, self-other overlap, and complex understanding in the formation of a new relationship. The Journal of Positive Psychology. Special Issue: Positive Emotions, 1(2), 93-106.

West, S. G., Finch, J. F., \& Curran, P. J. (1995). Structural equation models with nonnormal variables: Problems and remedies. In R. H. Hoyle (Ed.), Structural equation modeling: Concepts, issues, and application (pp. 56-75). Thousand Oaks, CA: Sage Publications.

Wheeler, R. E., Davidson, R. J., \& Tomarken, A. J. (1993). Frontal brain asymmetry and emotional reactivity: A biological substrate of affective style. Psychophysiology, 30(1), 82-89.

Wirtz, D., Kruger, J., Scollon, C.N., Diener, E. (2003). What to do on spring break? The role of predicted, on-line, and remembered experience in future choice. Psychological Science, 14(5), 520-524.

Wood, Heimpel, S. A., \& Michela, J. L. (2003). Savoring Versus Dampening: Self-Esteem Differences in Regulating Positive Affect. Journal of Personality and Social Psychology, 85(3), 566-580. 
Wood, A. M., Froh, J. J., \& Geraghty, A. W. A. (2010). Gratitude and well-being: A review and theoretical integration. Clinical Psychology Review, 30(7), 890-905.

Wrosch, C., \& Miller, G. E. (2009). Depressive symptoms can be useful: Self-regulatory and emotional benefits of dysphoric mood in adolescence. Journal of Personality and Social Psychology, 96(6), 1181-1190.

Xu, J., \& Roberts, R. E. (2010). The power of positive emotions: It's a matter of life or death: Subjective well-being and longevity over 28 years in a general population. Health Psychology, 29(1), 9-19.

Zealand, S. N. (2006). 2006 Census data: Quick statistics about New Zealand. Retrieved 14 September, 2010, from http://www.stats.govt.nz/Census/2006CensusHomePage/QuickStats/AboutAPlace/Sn apShot.aspx?type $=$ region\&tab $=$ Culturaldiversity\&id $=9999999$

Zika, S., \& Chamberlain, K. (1992). On the relation between meaning in life and psychological well-being. British Journal of Psychology, 83(133-145) 


\section{Appendix A: \\ Original Ways of Savoring Checklist ${ }^{33}$}

The following pages contain a list of things that people might think or do while they are going through positive events. Please read each of the following statements and indicate how much each of them applies to what you thought and did the last time you went on vacation.

When this event happened...

$\begin{array}{ccr}\text { Definitely } & & \\ \text { Doesn't } & \text { Applies } & \text { Definitely } \\ \text { Apply } & \text { Somewhat } & \text { Applies }\end{array}$

$\begin{array}{llllllllll}\text { 1. I thought about sharing the memory } & 1 & 2 & 3 & 4 & 5 & 6 & 7\end{array}$ of this later with other people.

2. I tried to take in every sensory property of the event (sights, sounds, smells, etc.).

3. I reminded myself how long I had waited for this to happen.

$\begin{array}{llllllllll}\text { 4. I reminded myself how transient this } & \text { I } & 2 & 3 & 4 & 5 & 6 & 7\end{array}$ moment was - thought about it ending.

5. I jumped up and down, ran around, $\quad \begin{array}{llllllllll}1 & 2 & 3 & 4 & 5 & 6 & 7\end{array}$ or showed other physical expressions of energy.

$\begin{array}{lllllllllll}\text { 6. I thought back to events that led up } & 1 & 2 & 3 & 4 & 5 & 6 & 7\end{array}$ to it - to a time when I didn't have it and wanted it.

7. I tried to focus on certain sensory $\quad \begin{array}{llllllllll} & 1 & 2 & 3 & 4 & 5 & 6 & 7\end{array}$ properties in particular (perhaps blocking out others).

8. I thought only about the present- $\quad \begin{array}{llllllllll} & 1 & 2 & 3 & 4 & 5 & 6 & 7\end{array}$ got absorbed in the moment.

9. I reminded myself how lucky I was $\quad \begin{array}{llllllllll}\text { 9. } & 1 & 2 & 3 & 4 & 5 & 6 & 7\end{array}$ to have this good thing happen to me.

10. I told myself why I didn't deserve this $\begin{array}{lllllllll}\text { ? } & 1 & 2 & 3 & 4 & 5 & 6 & 7\end{array}$ good thing. 
Definitely

Doesn't Applies Definitely

Apply Somewhat Applies

11. I looked for other people to share it $\begin{array}{llllllll}- & 1 & 2 & 3 & 4 & 5 & 6 & 7\end{array}$ with.

12. I thought about how I'd reminisce to $\begin{array}{llllllll}1 & 2 & 3 & 4 & 5 & 6 & 7\end{array}$ myself about this event later.

13. I reminded myself what a relief it was. $\begin{array}{llllllll}1 & 2 & 3 & 4 & 5 & 6 & 7\end{array}$

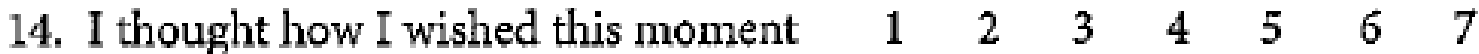
could last-reminded myself how I must enjoy it now because it would soon be over.

15. I laughed or giggled.

16. I thought about ways in which it $\begin{array}{lllllll}1 & 2 & 3 & 4 & 5 & 6 & 7\end{array}$ could have been worse.

17. I opened my eyes wide and took a $\begin{array}{lllllll}1 & 2 & 3 & 4 & 5 & 6 & 7\end{array}$ deep breath - tried to become more alert.

18. I closed my eyes, relaxed, took in the $\begin{array}{llllllll}1 & 2 & 3 & 4 & 5 & 6 & 7\end{array}$ moment.

$\begin{array}{llllllll}\text { 19. I thought about what a lucky person } & 1 & 2 & 3 & 4 & 5 & 6 & 7\end{array}$ I am that so many good things have happened to me.

20. I thought about ways in which it

$\begin{array}{lllllll}1 & 2 & 3 & 4 & 5 & 6 & 7\end{array}$

21. I expressed to others present how $\begin{array}{lllllll}1 & 2 & 3 & 4 & 5 & 6 & 7\end{array}$ much I valued the moment (and their being there to share it with me).

22. I consciously reflected on the situation $1 \begin{array}{llllllll} & 1 & 2 & 3 & 4 & 5 & 6 & 7\end{array}$ - took in details, tried to remember them, made comparisons.

23. I told myself how proud I was.

24. I reminded myself that it would be over before I knew it.

25. I tried to speed up and move more quickly.

$\begin{array}{lllllll}1 & 2 & 3 & 4 & 5 & 6 & 7 \\ 1 & 2 & 3 & 4 & 5 & 6 & 7 \\ 1 & 2 & 3 & 4 & 5 & 6 & 7\end{array}$


Definitely

Doesn't Applies Definitely

Apply Somewhat Applies

26. I focused on the future - on a time when this good event would be over.

27. I tried to slow down and move more $\begin{array}{llllllll}1 & 2 & 3 & 4 & 5 & 6 & 7\end{array}$ slowly (in an effort to stop or slow down time).

28. I made myself relax so that I could become more absorbed in the event

$\begin{array}{lllllll}1 & 2 & 3 & 4 & 5 & 6 & 7\end{array}$ or activity.

29. I said a prayer of thanks for my good $\quad \begin{array}{llllllll}1 & 2 & 3 & 4 & 5 & 6 & 7\end{array}$ fortune.

30. I withdrew and inhibited my feelings $\begin{array}{lllllllll}1 & 2 & 3 & 4 & 5 & 6 & 7\end{array}$ (stiffened up).

$\begin{array}{lllllllll}\text { 31. I hung around with others who know } & 1 & 2 & 3 & 4 & 5 & 6 & 7\end{array}$ how to have a good time.

32. I labeled specific details of the situation explicitly - tried to find out what it was that I was enjoying and note each aspect explicitly.

33. I told myself how impressed others $\quad$ I $\quad \begin{array}{lllllll}2 & 3 & 4 & 5 & 6 & 7\end{array}$ must be.

34. I reminded myself that nothing lasts $\quad \begin{array}{lllllll}1 & 2 & 3 & 4 & 5 & 6 & 7\end{array}$ forever so I must enjoy this now.

$\begin{array}{lllllllll}\text { 35. I sighed or made other verbal sounds } & 1 & 2 & 3 & 4 & 5 & 6 & 7\end{array}$ of appreciation to help myself savor the moment (e.g., saying $\mathrm{mmm}$, aahh, humming or whistling).

36. I reminded myself that others who $\quad \begin{array}{llllllll}1 & 2 & 3 & 4 & 5 & 6 & 7\end{array}$ were involved in the event were also 


$\begin{array}{ccr}\text { Definitely } & & \\ \text { Doesn't } & \text { Applies } & \text { Definitely } \\ \text { Apply } & \text { Somewhat } & \text { Applies }\end{array}$

37. I concentrated and blocked out $\begin{array}{lllllll}1 & 2 & 3 & 4 & 5 & 6 & 7\end{array}$ distractions; I intensified one sense by blocking another.

38. I just went through the experience one moment at a time and tried not to look too far ahead.

39. I told myself how it wasn't as good as $\quad \begin{array}{llllllll}1 & 2 & 3 & 4 & 5 & 6 & 7\end{array}$ I'd hoped for.

40. I physically expressed my feelings to $\begin{array}{llllllll}1 & 2 & 3 & 4 & 5 & 6 & 7\end{array}$ others (hugging, touching).

41. I took mental photographs.

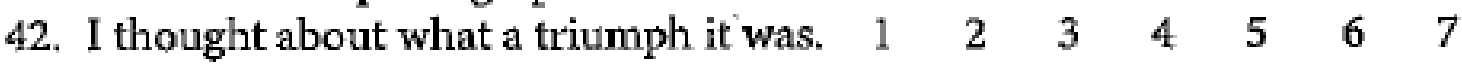

43. I thought about how fast the time was $\begin{array}{llllllll}1 & 2 & 3 & 4 & 5 & 6 & 7\end{array}$ passing.

44. I screamed or made other verbal $\quad \begin{array}{llllllll}1 & 2 & 3 & 4 & 5 & 6 & 7\end{array}$ expressions of excitement.

45. I compared myself to others (asked $\quad \begin{array}{llllllll}1 & 2 & 3 & 4 & 5 & 6 & 7\end{array}$ myself "Am I enjoying this as much as they are?").

46. I reminded myself of other places I $\quad \begin{array}{lllllllll} & 1 & 2 & 3 & 4 & 5 & 6 & 7\end{array}$ should be or of other things I should be doing instead.

47. I talked to another person about how $\quad \begin{array}{llllllll} & 1 & 2 & 3 & 4 & 5 & 6 & 7\end{array}$ good I felt.

48. I tried to memorize my surroundings. $\begin{array}{llllllll}1 & 2 & 3 & 4 & 5 & 6 & 7\end{array}$

49. I told myself why I deserved this $\quad \begin{array}{llllllll}1 & 2 & 3 & 4 & 5 & 6 & 7\end{array}$ good thing.

50. I touched myself-rubbed my stomach, clapped my hands, etc.

51. I made associations with other past $\quad \begin{array}{llllllll}1 & 2 & 3 & 4 & 5 & 6 & 7\end{array}$ pleasant events and reminded myself of them.

52. I thought about other things that $\quad \begin{array}{llllllll}1 & 2 & 3 & 4 & 5 & 6 & 7\end{array}$ were hanging over me, problems and worries that I still had to face.

53. I took photographs with a camera to $\begin{array}{llllllll}1 & 2 & 3 & 4 & 5 & 6 & 7\end{array}$ capture the experience.

54. I thought about what a good time I $\quad \begin{array}{llllllll}1 & 2 & 3 & 4 & 5 & 6 & 7\end{array}$ was having. 


\section{Definitely \\ Doesn't Applies Definitely \\ Apply Somewhat Applies}

55. I thought about how things might

$\begin{array}{lllllll}1 & 2 & 3 & 4 & 5 & 6 & 7\end{array}$

never be this good again.

56. I thought about things that made

$\begin{array}{lllllll}1 & 2 & 3 & 4 & 5 & 6 & 7\end{array}$

me feel guilty.

$\begin{array}{lllllllll}\text { 57. I thought or did something entirely } & 1 & 2 & 3 & 4 & 5 & 6 & 7\end{array}$ different from any of the above. (Please describe).

$\begin{array}{lllllllll}\text { 58. I got high or intoxicated to help me } & 1 & 2 & 3 & 4 & 5 & 6 & 7\end{array}$ enjoy it.

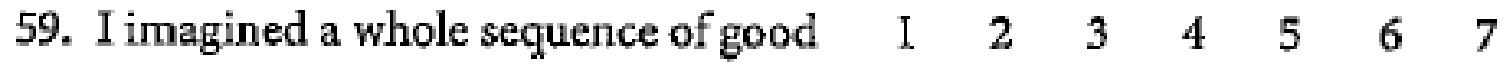
events that could arise as a consequence of this event.

60. I tried not to think too much - just $\quad \begin{array}{llllllll}1 & 2 & 3 & 4 & 5 & 6 & 7\end{array}$ relaxed and enjoyed. 


\section{Appendix B:}

\section{Everyday Positive Events Scale (Adolescents)}

Directions: Below is a list of events that can happen to anyone. Please read each and indicate if any of these has happened to you in the last month. If you answer "YES" also circle the number corresponding to "how much of a positive experience it was," from (0) None, to, (3) $A$ lot.

\begin{tabular}{|c|c|c|c|c|c|c|}
\hline \multirow{2}{*}{$\begin{array}{c}\text { Has this happen to You in the } \\
\text { last MONTH? }\end{array}$} & \multirow{2}{*}{ YES } & \multirow{2}{*}{ NO } & \multicolumn{4}{|c|}{$\begin{array}{l}\text { If you said "yes", how much of a } \\
\text { positive experience was it? }\end{array}$} \\
\hline & & & None & A little & Some & $A$ lot \\
\hline $\begin{array}{l}\text { A stranger was nice to you for no } \\
\text { reason }\end{array}$ & Y & $\mathrm{N}$ & 0 & 1 & 2 & 3 \\
\hline Got some unexpected money & $\mathrm{Y}$ & $\mathrm{N}$ & 0 & 1 & 2 & 3 \\
\hline $\begin{array}{l}\text { 3. Had a romantic time with your } \\
\text { boyfriend/girlfriend }\end{array}$ & Y & $\mathrm{N}$ & 0 & 1 & 2 & 3 \\
\hline You made a new friend & Y & $\mathrm{N}$ & 0 & 1 & 2 & 3 \\
\hline $\begin{array}{l}\text { 5. Something you dreaded turned } \\
\text { out to be easier than expected }\end{array}$ & Y & $\mathrm{N}$ & 0 & 1 & 2 & 3 \\
\hline Your fitness or health improved & Y & $\mathrm{N}$ & 0 & 1 & 2 & 3 \\
\hline $\begin{array}{l}\text { Had a stimulating conversation } \\
\text { with someone }\end{array}$ & Y & $\mathrm{N}$ & 0 & 1 & 2 & 3 \\
\hline $\begin{array}{l}\text { Experienced something new } \\
\text { 8. that was interesting }\end{array}$ & Y & $\mathrm{N}$ & 0 & 1 & 2 & 3 \\
\hline $\begin{array}{l}\text { 9. Laughed hard at something } \\
\text { funny }\end{array}$ & Y & $\mathrm{N}$ & 0 & 1 & 2 & 3 \\
\hline 10. Had a really wonderful meal & Y & $\mathrm{N}$ & 0 & 1 & 2 & 3 \\
\hline $\begin{array}{l}\text { Shared something funny with a } \\
\text { friend }\end{array}$ & Y & $\mathrm{N}$ & 0 & 1 & 2 & 3 \\
\hline $\begin{array}{l}\text { Did something that made you } \\
\text { 12. feel good/proud of yourself }\end{array}$ & $\mathrm{Y}$ & $\mathrm{N}$ & 0 & 1 & 2 & 3 \\
\hline $\begin{array}{l}\text { Did something that made you } \\
\text { 13. feel like you've grown-up }\end{array}$ & $\mathrm{Y}$ & $\mathrm{N}$ & 0 & 1 & 2 & 3 \\
\hline
\end{tabular}




\begin{tabular}{|c|c|c|c|c|c|c|}
\hline \multirow{2}{*}{$\begin{array}{c}\text { Has this happen to You in the } \\
\text { last MONTH? }\end{array}$} & \multirow{2}{*}{ YES } & \multirow{2}{*}{ NO } & \multicolumn{4}{|c|}{$\begin{array}{l}\text { If you said "yes", how much of a } \\
\text { positive experience was it? }\end{array}$} \\
\hline & & & None & A little & Some & A lot \\
\hline 14. You made a good decision & Y & $\mathrm{N}$ & 0 & 1 & 2 & 3 \\
\hline 15. Someone complimented you & $\mathrm{Y}$ & $\mathrm{N}$ & 0 & 1 & 2 & 3 \\
\hline $\begin{array}{l}\text { 16. Something happened to make } \\
\text { you excited about the future }\end{array}$ & $\mathrm{Y}$ & $\mathrm{N}$ & 0 & 1 & 2 & 3 \\
\hline $\begin{array}{l}\text { 17. Shared a good time with your } \\
\text { parents }\end{array}$ & $\mathrm{Y}$ & $\mathrm{N}$ & 0 & 1 & 2 & 3 \\
\hline 18. Cleared up a disagreement & Y & $\mathrm{N}$ & 0 & 1 & 2 & 3 \\
\hline $\begin{array}{l}\text { 19. Moved into a better living } \\
\text { place }\end{array}$ & Y & $\mathrm{N}$ & 0 & 1 & 2 & 3 \\
\hline $\begin{array}{l}\text { 20. Listened to music you really } \\
\text { like }\end{array}$ & $\mathrm{Y}$ & $\mathrm{N}$ & 0 & 1 & 2 & 3 \\
\hline 21. Read a book that excited you & Y & $\mathrm{N}$ & 0 & 1 & 2 & 3 \\
\hline 22. Saw a really good movie & Y & $\mathrm{N}$ & 0 & 1 & 2 & 3 \\
\hline 23. Helped someone & Y & $\mathrm{N}$ & 0 & 1 & 2 & 3 \\
\hline $\begin{array}{l}\text { 24. Were grateful for someone in } \\
\text { your life }\end{array}$ & $\mathrm{Y}$ & $\mathrm{N}$ & 0 & 1 & 2 & 3 \\
\hline $\begin{array}{l}\text { 25. Were grateful for your } \\
\text { upbringing }\end{array}$ & Y & $\mathrm{N}$ & 0 & 1 & 2 & 3 \\
\hline $\begin{array}{l}\text { Is there an event we missed, } \\
\text { 26. for example, a sporting win? } \\
\text { Please specify: }\end{array}$ & Y & $\mathrm{N}$ & 0 & 1 & 2 & 3 \\
\hline 27. Anything else we missed? & $\mathrm{Y}$ & $\mathrm{N}$ & 0 & 1 & 2 & 3 \\
\hline
\end{tabular}




\section{Appendix C:}

\section{Everyday Positive Events Scale (Adults)}

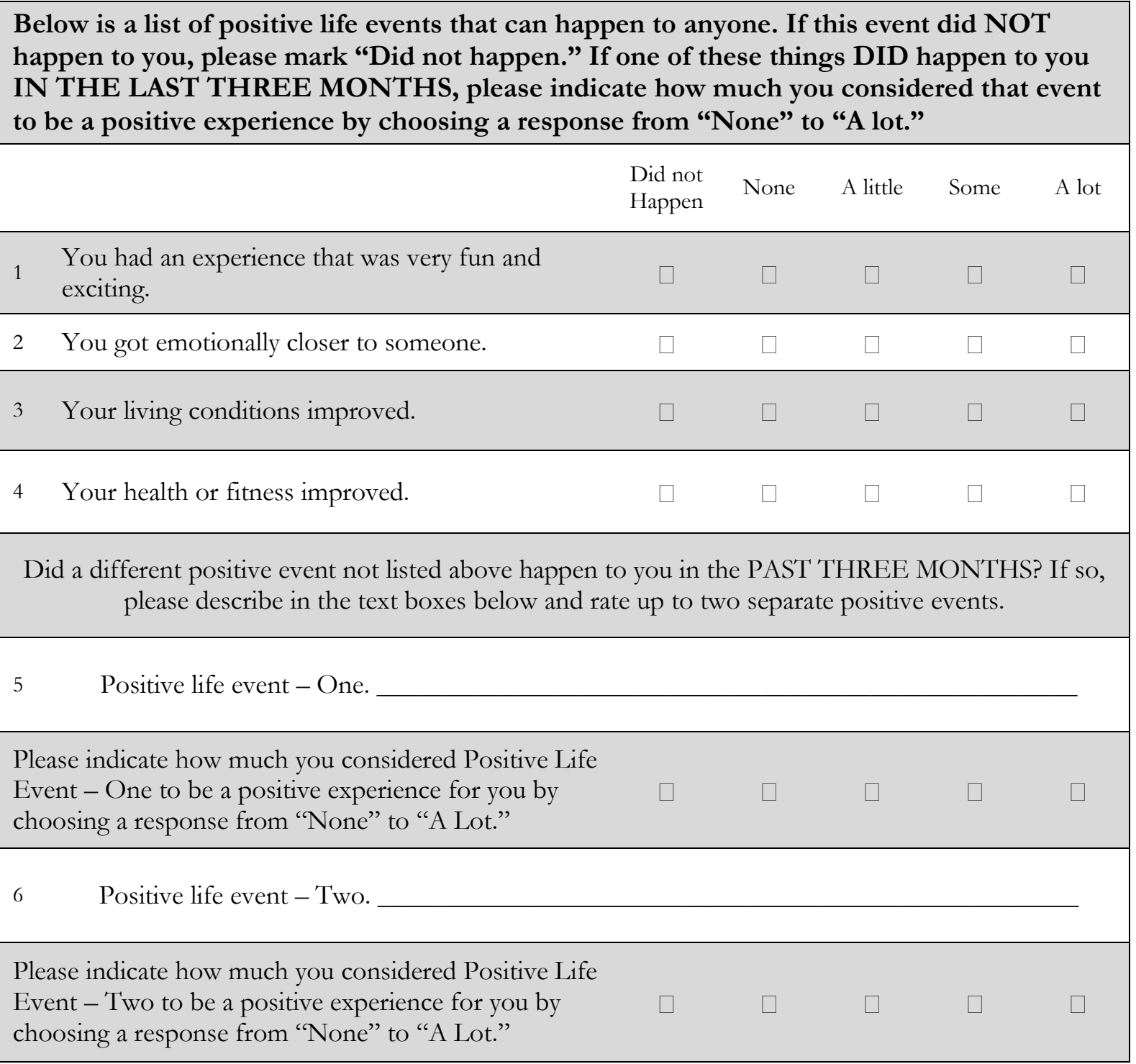




\section{Appendix D:}

\section{Abridged Ways of Savoring Checklist (Adolescents)}

Direction: Please think about the positive events you listed earlier. We are interested in how you responded to those events. Please read each statement and indicate how much each of them applies to how you responded to the events you listed, from 1 'Strongly Disagree' to 7 'Strongly Agree'. Remember there is no right or wrong answers.

How did you respond?

\section{Strongly}

Disagree
Strongly

Neutral

1. I thought about sharing the memory of this later $\begin{array}{lll}1 & 2 & 3\end{array}$ 4 7 with other people.

2. I tried to take in every sensory property of the event $\begin{array}{llllllllll}1 & 2 & 3 & 4 & 5 & 6 & 7\end{array}$ (sights, sounds, smells, etc.)

3. I reminded myself how transient this moment was - $\begin{array}{llllllllll}1 & 2 & 3 & 4 & 5 & 6 & 7\end{array}$ thought about it ending.

4. I jumped up and down, ran around or showed other $\begin{array}{llllllllll}1 & 2 & 3 & 4 & 5 & 6 & 7\end{array}$ physical expressions of energy.

5. I thought back to events that led up to it - to a time $\begin{array}{llllllllll}1 & 2 & 3 & 4 & 5 & 6 & 7\end{array}$ when I didn't have it and wanted it.

6. I thought only about the present - got absorbed in $\begin{array}{llllllllll}1 & 2 & 3 & 4 & 5 & 6 & 7\end{array}$ the moment.

7. I reminded myself how lucky I was to have this $\quad \begin{array}{lllllllllll}1 & 2 & 3 & 4 & 5 & 6 & 7\end{array}$ good thing happen to me.

8. I told myself why I didn't deserve this good thing. $\begin{array}{llllllllll}1 & 2 & 3 & 4 & 5 & 6 & 7\end{array}$

9. I looked for other people to share it with. $\quad \begin{array}{llllllllll} & 1 & 2 & 3 & 4 & 5 & 6 & 7\end{array}$

10. I thought about how I'd think to myself about this $\begin{array}{llllllllll}1 & 2 & 3 & 4 & 5 & 6 & 7\end{array}$ event later.

11. I laughed or giggled.

12. I opened my eyes wide and took a deep breath tried to be more alert.

13. I closed my eyes, relaxed, took in the moment. $\quad \begin{array}{llllllllll}1 & 2 & 3 & 4 & 5 & 6 & 7\end{array}$

14. I thought about what a lucky person I am that so $\quad \begin{array}{llllllllll}1 & 2 & 3 & 4 & 5 & 6 & 7\end{array}$ many good things have happened to me.

15. I thought about ways in which it could have been $\quad \begin{array}{lllllllll}1 & 2 & 3 & 4 & 5 & 6 & 7\end{array}$ better. 
17. I reminded myself that it would be over before I $\quad \begin{array}{llllllllll}1 & 2 & 3 & 4 & 5 & 6 & 7\end{array}$ knew it.

18. I focused on the future - on a time when this good $\quad \begin{array}{llllllllll}1 & 2 & 3 & 4 & 5 & 6 & 7\end{array}$ event would be over.

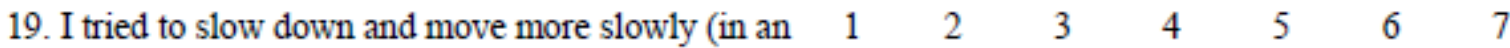
effort to stop or slow time).

20. I made myself relax so that I could become more $\quad \begin{array}{llllllllll}1 & 2 & 3 & 4 & 5 & 6 & 7\end{array}$ absorbed in the event or activity.

21. I said a prayer of thanks for my good fortune. $\quad \begin{array}{llllllllll}1 & 2 & 3 & 4 & 5 & 6 & 7\end{array}$

22. I told myself how impressed others must be. $\quad \begin{array}{llllllllll}7 & 2 & 3 & 4 & 5 & 6 & 7\end{array}$

23. I reminded myself that nothing lasts forever. $\quad \begin{array}{llllllllll}1 & 2 & 3 & 4 & 5 & 6 & 7\end{array}$

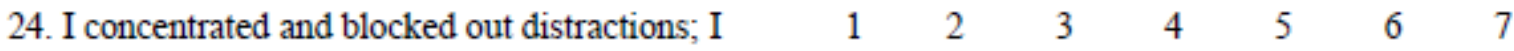
intensified one sense by blocking another.

25. I told myself how it wasn't as good as I'd hoped $\quad \begin{array}{lllllllll}7 & 2 & 3 & 4 & 5 & 6 & 7\end{array}$ for.

26. I screamed or made other verbal expressions of $\quad \begin{array}{llllllllll}1 & 2 & 3 & 4 & 5 & 6 & 7\end{array}$ excitement.

27. I talked to another person about how good I felt. $\quad \begin{array}{llllllllll}1 & 2 & 3 & 4 & 5 & 6 & 7\end{array}$

28. I tried to memorise my surroundings. $\quad \begin{array}{lllllllllll}1 & 2 & 3 & 4 & 5 & 6 & 7\end{array}$

29. I told myself why I deserved this good thing. $\quad \begin{array}{llllllllll}7 & 2 & 3 & 4 & 5 & 6 & 7\end{array}$

30. I thought about how things might never be this $\quad \begin{array}{llllllllll}1 & 2 & 3 & 4 & 5 & 6 & 7\end{array}$ good again.

Adapted from:

Bryant, F. B. \& Veroff, J. (2007). Savoring: A New Model of Positive Experience. NJ:

Lawrence Erlbaum Associates. 


\section{Appendix E:}

\section{Abridged Ways of Savoring Checklist (Adults)}

Please think about positive events you have experience in the past three months, and how you responded to those events, and answer the following.

\begin{tabular}{|c|c|c|c|c|c|c|}
\hline $\begin{array}{l}\text { Strongly } \\
\text { disagree }\end{array}$ & Disagree & $\begin{array}{l}\text { Slightly } \\
\text { disagree }\end{array}$ & $\begin{array}{l}\text { Neither } \\
\text { agree } \\
\text { nor } \\
\text { disagree }\end{array}$ & $\begin{array}{l}\text { Slightly } \\
\text { agree }\end{array}$ & Agree & $\begin{array}{c}\text { Strongly } \\
\text { agree }\end{array}$ \\
\hline
\end{tabular}

\begin{tabular}{|c|c|c|c|c|c|c|c|c|}
\hline 1 & $\begin{array}{l}\text { I thought about sharing the } \\
\text { memory of this later with } \\
\text { other people }\end{array}$ & $\square$ & $\square$ & $\square$ & $\square$ & $\square$ & $\square$ & $\square$ \\
\hline 2 & $\begin{array}{l}\text { I tried to take in every sensory } \\
\text { property of the event (sights, } \\
\text { sounds, smells, etc.) }\end{array}$ & $\square$ & $\square$ & $\square$ & $\square$ & $\square$ & $\square$ & $\square$ \\
\hline 3 & $\begin{array}{l}\text { I reminded myself how } \\
\text { transient this moment was- } \\
\text { thought about it ending. }\end{array}$ & $\square$ & $\square$ & $\square$ & $\square$ & $\square$ & $\square$ & $\square$ \\
\hline 4 & $\begin{array}{l}\text { I jumped up and down, ran } \\
\text { around, or showed other } \\
\text { physical expressions of energy. }\end{array}$ & $\square$ & $\square$ & $\square$ & $\square$ & $\square$ & $\square$ & $\square$ \\
\hline 5 & $\begin{array}{l}\text { I thought back to events that } \\
\text { led up to it- to a time when I } \\
\text { didn't have it and wanted it. }\end{array}$ & $\square$ & $\square$ & $\square$ & $\square$ & $\square$ & $\square$ & $\square$ \\
\hline 6 & $\begin{array}{l}\text { I thought only about the } \\
\text { present—got absorbed in the } \\
\text { moment. }\end{array}$ & $\square$ & $\square$ & $\square$ & $\square$ & $\square$ & $\square$ & $\square$ \\
\hline 7 & $\begin{array}{l}\text { I reminded myself how lucky I } \\
\text { was to have this good thing } \\
\text { happen to me. }\end{array}$ & $\square$ & $\square$ & $\square$ & $\square$ & $\square$ & $\square$ & $\square$ \\
\hline 8 & $\begin{array}{l}\text { I told myself why I didn't } \\
\text { deserve this good thing. }\end{array}$ & $\square$ & $\square$ & $\square$ & $\square$ & $\square$ & $\square$ & $\square$ \\
\hline 9 & $\begin{array}{l}\text { I looked for other people to } \\
\text { share it with. }\end{array}$ & $\square$ & $\square$ & $\square$ & $\square$ & $\square$ & $\square$ & $\square$ \\
\hline 10 & $\begin{array}{l}\text { I thought about how I'd think } \\
\text { to myself about this event } \\
\text { later. }\end{array}$ & $\square$ & $\square$ & $\square$ & $\square$ & $\square$ & $\square$ & $\square$ \\
\hline 11 & I laughed or giggled & $\square$ & $\square$ & $\square$ & $\square$ & $\square$ & $\square$ & $\square$ \\
\hline 12 & $\begin{array}{l}\text { I opened my eyes wide and } \\
\text { took a deep breath—-tried to } \\
\text { become more alert. }\end{array}$ & $\square$ & $\square$ & $\square$ & $\square$ & $\square$ & $\square$ & $\square$ \\
\hline 13 & $\begin{array}{l}\text { I closed my eyes, relaxed, took } \\
\text { in the moment. }\end{array}$ & $\square$ & $\square$ & $\square$ & $\square$ & $\square$ & $\square$ & $\square$ \\
\hline 14 & $\begin{array}{l}\text { I thought about what a lucky } \\
\text { person I am that so many } \\
\text { good things have happened to } \\
\text { me. }\end{array}$ & $\square$ & $\square$ & $\square$ & $\square$ & $\square$ & $\square$ & $\square$ \\
\hline
\end{tabular}




\begin{tabular}{|c|c|c|c|c|c|c|c|c|}
\hline 15 & $\begin{array}{l}\text { I thought about ways in which } \\
\text { it could have been better. }\end{array}$ & $\square$ & $\square$ & $\square$ & $\square$ & $\square$ & $\square$ & $\square$ \\
\hline 16 & $\begin{array}{l}\text { I told myself how proud I } \\
\text { was. }\end{array}$ & $\square$ & $\square$ & $\square$ & $\square$ & $\square$ & $\square$ & $\square$ \\
\hline 17 & $\begin{array}{l}\text { I reminded myself that it } \\
\text { would be over before I knew } \\
\text { it. }\end{array}$ & $\square$ & $\square$ & $\square$ & $\square$ & $\square$ & $\square$ & $\square$ \\
\hline 18 & $\begin{array}{l}\text { I focussed on the future-on } \\
\text { a time when this good event } \\
\text { would be over. }\end{array}$ & $\square$ & $\square$ & $\square$ & $\square$ & $\square$ & $\square$ & $\square$ \\
\hline 19 & $\begin{array}{l}\text { I tried to slow down and } \\
\text { move more slowly (in an } \\
\text { effort to stop or slow time). }\end{array}$ & $\square$ & $\square$ & $\square$ & $\square$ & $\square$ & $\square$ & $\square$ \\
\hline 20 & $\begin{array}{l}\text { I told myself how impressed } \\
\text { others must be. }\end{array}$ & $\square$ & $\square$ & $\square$ & $\square$ & $\square$ & $\square$ & $\square$ \\
\hline
\end{tabular}




\section{Appendix F:}

\section{Satisfaction with Life Scale}

Directions: Below are five statements with which you may agree or disagree. Circle the point on the scale that indicates your agreement or disagreement with each item, from (1) to (7).

Please be open and honest in your responding.

1. In most ways my life is close to my ideal

Strongly

Disagree

Neutral

Strongly

1

2

3

4

$5 \quad 6$

7

2. The conditions of my life are excellent

Strongly

Disagree

Neutral

Strongly

12

3

4

5

6

7

3. I am satisfied with my life

Strongly

Disagree

Neutral

Strongly

Agree

1

2

3

4

5

6

7

4. So far I have gotten the important things I want in life

Strongly

Disagree

Neutral

Strongly

1

2

3

4

5

6

7

5. If I could live my life over, I would change almost nothing

Strongly

Disagree

Neutral

Strongly

1

2

3

4

5

6

7 


\section{Appendix G:}

\section{Temporal Satisfaction with Life Scale}

\section{Below are statements that many people would find desirable, but we want you to answer only in terms of whether the statement describes HOW YOU ACTUALLY LIVE YOUR LIFE.}

\begin{tabular}{|c|c|c|c|c|c|c|c|c|}
\hline & & $\begin{array}{l}\text { Strongly } \\
\text { disagree }\end{array}$ & Disagree & $\begin{array}{l}\text { Slightly } \\
\text { disagree }\end{array}$ & $\begin{array}{l}\text { Neither } \\
\text { agree } \\
\text { nor } \\
\text { disagree }\end{array}$ & $\begin{array}{l}\text { Slightly } \\
\text { agree }\end{array}$ & Agree & $\begin{array}{c}\text { Strongly } \\
\text { agree }\end{array}$ \\
\hline 1 & $\begin{array}{l}\text { If I had my past to live } \\
\text { over, I would change } \\
\text { nothing. }\end{array}$ & $\square$ & $\square$ & $\square$ & $\square$ & $\square$ & $\square$ & $\square$ \\
\hline 2 & $\begin{array}{l}\text { I am satisfied with my life } \\
\text { in the past. }\end{array}$ & $\square$ & $\square$ & $\square$ & $\square$ & $\square$ & $\square$ & $\square$ \\
\hline 3 & $\begin{array}{l}\text { My life in the past was } \\
\text { ideal for me. }\end{array}$ & $\square$ & $\square$ & $\square$ & $\square$ & $\square$ & $\square$ & $\square$ \\
\hline 4 & $\begin{array}{l}\text { The conditions of my life } \\
\text { in the past were excellent. }\end{array}$ & $\square$ & $\square$ & $\square$ & $\square$ & $\square$ & $\square$ & $\square$ \\
\hline 5 & $\begin{array}{l}\text { I had the important things } \\
\text { I wanted in my past. }\end{array}$ & $\square$ & $\square$ & $\square$ & $\square$ & $\square$ & $\square$ & $\square$ \\
\hline 6 & $\begin{array}{l}\text { I would change nothing } \\
\text { about my current life. }\end{array}$ & $\square$ & $\square$ & $\square$ & $\square$ & $\square$ & $\square$ & $\square$ \\
\hline 7 & $\begin{array}{l}\text { I am satisfied with my } \\
\text { current life. }\end{array}$ & $\square$ & $\square$ & $\square$ & $\square$ & $\square$ & $\square$ & $\square$ \\
\hline 8 & $\begin{array}{l}\text { My current life is ideal for } \\
\text { me. }\end{array}$ & $\square$ & $\square$ & $\square$ & $\square$ & $\square$ & $\square$ & $\square$ \\
\hline 9 & $\begin{array}{l}\text { The current conditions of } \\
\text { my life are excellent. }\end{array}$ & $\square$ & $\square$ & $\square$ & $\square$ & $\square$ & $\square$ & $\square$ \\
\hline 10 & $\begin{array}{l}\text { I have the important things } \\
\text { I want right now. }\end{array}$ & $\square$ & $\square$ & $\square$ & $\square$ & $\square$ & $\square$ & $\square$ \\
\hline 11 & $\begin{array}{l}\text { There will be nothing that } \\
\text { I will want to change about } \\
\text { my future. }\end{array}$ & $\square$ & $\square$ & $\square$ & $\square$ & $\square$ & $\square$ & $\square$ \\
\hline 12 & $\begin{array}{l}\text { I will be satisfied with my } \\
\text { life in the future. }\end{array}$ & $\square$ & $\square$ & $\square$ & $\square$ & $\square$ & $\square$ & $\square$ \\
\hline 13 & $\begin{array}{l}\text { I expect my future life will } \\
\text { be ideal for me. }\end{array}$ & $\square$ & $\square$ & $\square$ & $\square$ & $\square$ & $\square$ & $\square$ \\
\hline 14 & $\begin{array}{l}\text { The conditions of my } \\
\text { future life will be excellent. }\end{array}$ & $\square$ & $\square$ & $\square$ & $\square$ & $\square$ & $\square$ & $\square$ \\
\hline 15 & $\begin{array}{l}\text { I will have the important } \\
\text { things I want in the future. }\end{array}$ & $\square$ & $\square$ & $\square$ & $\square$ & $\square$ & $\square$ & $\square$ \\
\hline
\end{tabular}




\section{Appendix H: \\ Subjective Happiness Scale}

Directions: For each of the following statements and/or questions, please circle the point on the scale from (1) to (7) that you feel is most appropriate in describing you.

1. In general, I consider myself:

Not a very happy person

A very bappy person

\begin{tabular}{llllllll}
\hline 1 & 2 & 3 & 4 & 5 & 6 & 7
\end{tabular}

2. Compared to most of my peers, I consider myself:

Less happy

More bappy

$\begin{array}{llllllll}1 & 2 & 3 & 4 & 5 & 6 & 7\end{array}$

Some people are generally very happy. They enjoy life regardless of what is going

3. on, getting the most out of everything. To what extent does this characterization describe you?

Not at all

A great deal

$\begin{array}{lllllll}1 & 2 & 3 & 4 & 5 & 6 & 7\end{array}$

Some people are generally not very happy. Although they are not depressed, they

4. never seem as happy as they might be. To what extent does this characterization describe you?

Not at all A great deal

1

2

3

4

5

6 7 


\section{Appendix I:}

\section{Mental Health Short Form}

Directions: For each of the following, please tick how often you had felt the corresponding way in the last month, from (1) Never to (6) Every Day.

\begin{tabular}{|c|c|c|c|c|c|c|}
\hline & Never & $\begin{array}{c}\text { Once or } \\
\text { twice a } \\
\text { week }\end{array}$ & $\begin{array}{c}\text { About } \\
\text { once a } \\
\text { week }\end{array}$ & $\begin{array}{c}\text { Two or } \\
\text { three } \\
\text { times a } \\
\text { week }\end{array}$ & $\begin{array}{l}\text { Almost } \\
\text { every day }\end{array}$ & Every day \\
\hline 1. Нарру & $\square$ & $\square$ & $\square$ & $\square$ & $\square$ & $\square$ \\
\hline 2. Interested in life & $\square$ & $\square$ & $\square$ & $\square$ & $\square$ & $\square$ \\
\hline 3. Satisfied & $\square$ & $\square$ & $\square$ & $\square$ & $\square$ & $\square$ \\
\hline
\end{tabular}

4. Good at managing the responsibilities of your daily life?

5. That you have warm and trusting relationships with other kids?

6. That you had experiences that challenged you to grow or become a better person?

7. Confident to think or express your own ideas and opinions?

8. That you had something important to contribute to society?

9. That you belonged to a community like a social group, your school, or your neighborhood?

10. That our society is becoming a better place?

11. That people are basically good?

12. That the way our society works made sense to you? 


\section{Appendix J: \\ Orientations to Happiness Scale ${ }^{34}$}

Below are statements that many people would find desirable, but we want you to answer only in terms of whether the statement describes HOW YOU ACTUALLY LIVE YOUR LIFE.

\begin{tabular}{|c|c|c|c|c|c|c|}
\hline & & $\begin{array}{l}\text { Not like } \\
\text { me at all } \\
\text { like me }\end{array}$ & $\begin{array}{l}\text { A little } \\
\text { like me }\end{array}$ & $\begin{array}{l}\text { Some- } \\
\text { what like } \\
\text { me }\end{array}$ & $\begin{array}{l}\text { Mostly } \\
\text { like me }\end{array}$ & $\begin{array}{l}\text { Very } \\
\text { much } \\
\text { like } \\
\text { me }\end{array}$ \\
\hline 1 & $\begin{array}{l}\text { Regardless of what I am doing, time } \\
\text { passes very quickly. }\end{array}$ & $\square$ & $\square$ & $\square$ & $\square$ & $\square$ \\
\hline 2 & My life serves a higher purpose. & $\square$ & $\square$ & $\square$ & $\square$ & $\square$ \\
\hline 3 & $\begin{array}{l}\text { Life is too short to postpone the } \\
\text { pleasures it can provide. }\end{array}$ & $\square$ & $\square$ & $\square$ & $\square$ & $\square$ \\
\hline 4 & $\begin{array}{l}\text { I seek out situations that challenge } \\
\text { my skills and abilities. }\end{array}$ & $\square$ & $\square$ & $\square$ & $\square$ & $\square$ \\
\hline 5 & $\begin{array}{l}\text { In choosing what to do, I always take } \\
\text { into account } \\
\text { whether it will benefit other people. }\end{array}$ & $\square$ & $\square$ & $\square$ & $\square$ & $\square$ \\
\hline 6 & $\begin{array}{l}\text { Whether at work or play, I am usually } \\
\text { "in a zone" and not conscious of } \\
\text { myself. }\end{array}$ & $\square$ & $\square$ & $\square$ & $\square$ & $\square$ \\
\hline 7 & $\begin{array}{l}\text { I am always very absorbed in what I } \\
\text { do. }\end{array}$ & $\square$ & $\square$ & $\square$ & $\square$ & $\square$ \\
\hline 8 & I go out of my way to feel euphoric & $\square$ & $\square$ & $\square$ & $\square$ & $\square$ \\
\hline 9 & $\begin{array}{l}\text { In choosing what to do, I always take } \\
\text { into account whether I can lose } \\
\text { myself in it. }\end{array}$ & $\square$ & $\square$ & $\square$ & $\square$ & $\square$ \\
\hline 10 & $\begin{array}{l}\text { I am rarely distracted by what is } \\
\text { going on around me. }\end{array}$ & $\square$ & $\square$ & $\square$ & $\square$ & $\square$ \\
\hline 11 & $\begin{array}{l}\text { I have a responsibility to make the } \\
\text { world a better place. }\end{array}$ & $\square$ & $\square$ & $\square$ & $\square$ & $\square$ \\
\hline 12 & My life has a lasting meaning. & $\square$ & $\square$ & $\square$ & $\square$ & $\square$ \\
\hline 13 & $\begin{array}{l}\text { In choosing what to do, I always take } \\
\text { into account whether it will be } \\
\text { pleasurable. }\end{array}$ & $\square$ & $\square$ & $\square$ & $\square$ & $\square$ \\
\hline 14 & What I do matters to society. & $\square$ & $\square$ & $\square$ & $\square$ & $\square$ \\
\hline 15 & $\begin{array}{l}\text { I agree with this statement: "Life is } \\
\text { short-eat dessert first." }\end{array}$ & $\square$ & $\square$ & $\square$ & $\square$ & $\square$ \\
\hline
\end{tabular}

34 The Meaning Orientation to Happiness includes items: 2, 5, 11, 12, 14, 17. The Pleasure Orientation to Happiness includes items: 3, 8, 13, 15, 16, 18. The Engagement Orientation to Happiness includes items: 1, 4, 6, 7, 9,10 . 


\begin{tabular}{|lllll}
\hline $16 \quad \begin{array}{l}\text { I love to do things that excite my } \\
\text { senses }\end{array}$ & $\square$ & $\square$ & $\square$ & $\square$ \\
\hline $17 \quad \begin{array}{l}\text { I have spent a lot of time thinking } \\
\text { about what life means and how I fit } \\
\text { into its big picture. }\end{array}$ & $\square$ & $\square$ & $\square$ & $\square$
\end{tabular}




\section{Appendix K: \\ Positive Negative Affective Schedule}

Directions: Here are a number of words that describe different feelings and emotions. Read each item and then tick to what extent you have felt this way during the past month, from (1) Very slightly or not at all, to, (5) Extremely.

Very slightly

or not at all A little Moderately Quite a bit Extremely

1. Interested

$\square \quad \square$

$\square$

$\square$

2. Distressed

3. Excited

$\square \quad \square$

$\square$

$\square$

4. Upset

5. Strong

$\square$

$\square$

$\square$

6. Guilty

7. Scared

$\square \quad \square$

$\square$

8. Hostile

9. Enthusiastic

$\square \quad \square$

$\square$

$\square$

10. Proud

11. Irritable

$\square \quad \square$

$\square$

$\square$

12. Alert

13. Ashamed

$\square \quad \square$

$\square \quad \square$

$\square \quad \square$

$\square$

14. Inspired

15. Nervous

$\square \quad \square$

$\square$

$\square$

16. Determined

17. Attentive $\square$

$\square$

18. Jittery

19. Active

$\square$

20. Afraid 